\title{
ON PETERSON'S OPEN PROBLEM AND REPRESENTATIONS OF THE GENERAL LINEAR GROUPS
}

\author{
ĐĂNG VÕ PHÚC \\ Dedicated to Professor Frank Williams
}

\begin{abstract}
Fix $\mathbb{Z} / 2$ is the prime field of two elements and write $\mathcal{A}_{2}$ for the mod 2 Steenrod algebra. Denote by $G L_{d}:=G L(d, \mathbb{Z} / 2)$ the general linear group of rank $d$ over $\mathbb{Z} / 2$ and by $\mathscr{P}_{d}$ the polynomial algebra $\mathbb{Z} / 2\left[x_{1}, x_{2}, \ldots, x_{d}\right]$, which is viewed as a connected unstable $\mathcal{A}_{2}$-module on $d$ generators of degree one. We study the Peterson "hit problem" of finding the minimal set of $\mathcal{A}_{2}$-generators for $\mathscr{P}_{d}$. It is equivalent to determining a $\mathbb{Z} / 2$-basis for the space of "cohits"

$$
Q \mathscr{P}_{d}:=\mathbb{Z} / 2 \otimes_{\mathcal{A}_{2}} \mathscr{P}_{d} \cong \mathscr{P}_{d} / \mathcal{A}_{2}^{+} \mathscr{P}_{d}
$$

This $Q \mathscr{P}_{d}$ is considered as a form modular representation of $G L_{d}$ over $\mathbb{Z} / 2$. The problem for $d=5$ is not yet completely solved, and unknown in general. In this work, we give an explicit solution to the hit problem of five variables in the generic degree $n=r\left(2^{t}-1\right)+2^{t} s$ with $r=d=5, s=8$ and $t$ an arbitrary non-negative integer. An application of this study to the cases $t=0$ and $t=1$ shows that the Singer algebraic transfer is an isomorphism in the bidegrees $\left(5,5+\left(13.2^{0}-5\right)\right)$ and $\left(5,5+\left(13.2^{1}-5\right)\right)$. Moreover, the result when $t \geq 2$ was also discussed. Here, the Singer transfer of rank $d$ is a $\mathbb{Z} / 2$-algebra homomorphism from $G L_{d^{d} \text {-coinvariants of }}$ certain subspaces of $Q \mathscr{P}_{d}$ to the cohomology groups of the Steenrod algebra, Ext ${ }_{\mathcal{A}_{2}}^{d, d+*}(\mathbb{Z} / 2, \mathbb{Z} / 2)$. It is one of the useful tools for studying mysterious Ext groups and the Kervaire invariant one problem.
\end{abstract}

\section{Contents}

1. Introduction and statement of results 1

2. Preliminaries 6

2.1. Kameko's squaring operation 6

2.2. On the classical "hit problem" of Peterson $\quad 7$

3. Generators of the $\mathcal{A}_{2}$-module $\mathscr{P}_{5}$ in the generic degree $5\left(2^{t}-1\right)+8.2^{t} \quad 9$

3.1. Singer's criterion on $\mathcal{A}_{2}$-decomposable $\quad 9$

3.2. Some homomorphisms and Sum's conjecture $\quad 9$

3.3. Proof of Theorem $1.1 \quad 11$

$\begin{array}{ll}\text { Computation of }\left(Q \mathscr{P}_{5}\right)_{22} & 14\end{array}$

Structure of the kernel of Kameko's map $\left(\widetilde{S q_{*}^{0}}\right)_{(5,47)} 17$

4. An application of Theorem $1.1 \quad 27$

4.1. Computation of $\left(Q \mathscr{P}_{5}\right)_{8}^{G L_{5}} \quad 28$

4.2. Computation of $\left(\operatorname{Ker}\left(\widetilde{S q_{*}^{0}}\right)_{(5,21)}\right)^{S_{5}} \quad 30$

4.3. Proof of Theorem $1.2 \quad 32$

5. Proof of Theorem 1.3 232

$\begin{array}{ll}\text { References } & 33\end{array}$

\section{Introduction and statement of results}

Throughout this article, we shall work only at the prime 2 . Let $S q^{k}: H^{*}(\mathbb{X}) \rightarrow H^{k+*}(\mathbb{X})$ be the stable cohomology operation of degree $k \geq 0$, which is introduced by Steenrod in 1947 (see $[60])$. Here $H^{*}(\mathbb{X})$ is the singular cohomology group of the topological space $\mathbb{X}$ with coefficients in $\mathbb{Z} / 2$. The $\mathbb{Z} / 2$-graded algebra $\mathcal{A}_{2}$ generated by the operations $S q^{k}$ is called the mod 2 Steenrod algebra and acts in a natural way on the cohomology of any space $\mathbb{X}$. For $d$ a natural number, we denote by $B(\mathbb{Z} / 2)^{\times d}$ the classifying space of elementary abelian 2 -group $(\mathbb{Z} / 2)^{\times d}$ of rank $d$ and by $\mathscr{P}_{d}=\mathbb{Z} / 2\left[x_{1}, x_{2}, \ldots, x_{d}\right]$ the polynomial algebra on $d$ variables of degree 1 . Of course, $B(\mathbb{Z} / 2)^{\times d}$ is homotopy equivalent to $(\mathbb{R} P(\infty))^{\times d}$, where $\mathbb{R} P(\infty)$ denotes the infinite real projective space. Since $\mathscr{P}_{d}$ is isomorphic to the cohomology with $\mathbb{Z} / 2$-coefficients of $B(\mathbb{Z} / 2)^{\times d}$, it has a connected unstable left $\mathcal{A}_{2}$-module structure. The left action of $\mathcal{A}_{2}$ on $\mathscr{P}_{d}$ is determined by the unstable condition $S q^{1}\left(x_{i}\right)=x_{i}^{2}, S q^{k}\left(x_{i}\right)=0$ for $k>1$ and Cartan's formula (see [60]).

The investigation of the homotopy classification of topological spaces leads us to the study of the cohomology groups of the Steenrod algebra, $\operatorname{Ext}_{\mathcal{A}_{2}}^{d, *}(\mathbb{Z} / 2, \mathbb{Z} / 2)$. It has been thoroughly studied for 
homological degrees $d \leq 5$ (see Adams [2], Adem [3], Wall [74], Wang [75], Tangora [68], Lin [23], Chen [10]). However, for $d$ higher, the calculations seem to be difficult. Moreover, it has a deep connection with the "hit problem" of our interest in determining the minimal set of $\mathcal{A}_{2}$-generators for $\mathscr{P}_{d}$. Equivalently, we need to find the dimension of the quotient space

$$
Q \mathscr{P}_{d}:=\mathbb{Z} / 2 \otimes_{\mathcal{A}_{2}} \mathscr{P}_{d} \cong \mathscr{P}_{d} / \mathcal{A}_{2}^{+} \mathscr{P}_{d}
$$

in each $d$ and degree $n \geq 1$. Here $\mathcal{A}_{2}^{+}$denotes the augmentation ideal of $\mathcal{A}_{2}$ and $\mathbb{Z} / 2$ is viewed as a right $\mathcal{A}_{2}$-module concentrated in grading 0 . This $Q \mathscr{P}_{d}$ will also be called the space of "cohits". The hit problem was posed by Peterson [37] in 1987. However, it remains open for $d \geq 5$.

As well known, the general linear group $G L_{d}:=G L(d, \mathbb{Z} / 2)$ acts regularly on $\mathscr{P}_{d}$ by matrix substitution. Further, the two actions of $\mathcal{A}_{2}$ and $G L_{d}$ upon $\mathscr{P}_{d}$ commute with each other; hence there is an inherited action of $G L_{d}$ on $Q \mathscr{P}_{d}$. From this event, one of the applications of the hit problem of Peterson is to study the representations of the general linear groups over $\mathbb{Z} / 2$. Therefrom the hit problem has attracted great interest of many algebraic topologists (see Crabb and Hubbuck [12], Crossley [13], Kameko [21], Mothebe and his collaborators [27, 28, 29], Nam [30], Pengelley and Williams [33, 35], Priddy [53], Silverman and Singer [56], Singer [58], Peterson [38], the present author [39, 40, 41, 42, 43, 45, 46, 49, 51, 52], Sum [61, 62, 63, 64, 65, 66, 67], Walker and Wood $[71,72,73]$, Wood [76, 77] and others).

Several other aspects of the hit problems were then studied by many authors. For instance, the hit problem for the symmetric of polynomials $\mathscr{P}_{d}^{S_{d}}$ as an $\mathcal{A}_{2}$-submodule of $\mathscr{P}_{d}$, has been of interest in [20], where $S_{d}$ is the symmetric group on $d$ letters acting on the right of $\mathscr{P}_{d}$, and $\mathscr{P}_{d}^{S_{d}}$ is isomorphic to the cohomology algebra $H^{*}(B O(d))$ of the Grassmannian of $d$-dimensional vector subspaces of $\mathbb{R} P(\infty)$. The space $B O(d)$ is the classifying space of the orthogonal group $O(d)$, Note also that the symmetric polynomials in $d$ variables divisible by all of them can be identified with the cohomology algebra of the Thom space $M O(d)$ of the standard $d$-plane bundle over $B O(d)$ associated with the bordism theory of closed smooth manifolds. In [17, 18], Hưng and his collaborators have studied the hit problem for the rank $d$ Dickson algebra, $\mathscr{P}_{d}^{G L_{d}}$ (the algebra of $G L_{d}$-invariants). The Dickson algebra is also an unstable $\mathcal{A}_{2}$-module and is dual to the coalgebra of Dyer-Lashof operations of the length $d$ (see Madsen [25]). The relationship between Kudo-ArakiMay algebra and the hit problem has been investigated by Pengelley and Williams [32, 34, 36], and by Singer [59]. In [5], Ault and Singer have examined the dual problem of the Peterson hit problem, which is to determine the set of $\mathcal{A}_{2}^{+}$-annihilated elements in the homology of $B(\mathbb{Z} / 2)^{\times d}$. Recently, Zare [78] has used geometric methods to study the hit problem for $H_{*}\left(B(\mathbb{Z} / 2)^{\times d}\right.$ ) (the dual of the hit problem of Peterson) as well as the hit problem for $H_{*}(B O(d))$ (the dual of the symmetric hit problem of Janfada and Wood). His main idea is based on the relation between the Dyer-Lashof algebra and these dual hit problems. Let $P_{\mathcal{A}_{2}} H_{*}\left(B(\mathbb{Z} / 2)^{\times d}\right)$ be the subspace of $H_{*}\left(B(\mathbb{Z} / 2)^{\times d}\right)$ consisting of all elements that are $\mathcal{A}_{2}^{+}$-annihilated. With the idea of describing the cohomology groups of the Steenrod algebra by means of modular representations of the general linear groups, William Singer [57] constructed a transfer homomorphism of rank $d$ from $G L_{d}$-coinvariants of the $\mathcal{A}_{2}^{+}$-annihilated elements of the dual of $\mathscr{P}_{d}$ to the cohomology of the Steenrod algebra:

$$
\operatorname{Tr}_{d}: \mathbb{Z} / 2 \otimes_{G L_{d}} P_{\mathcal{A}_{2}} H_{*}\left(B(\mathbb{Z} / 2)^{\times d}\right) \rightarrow \operatorname{Ext}_{\mathcal{A}_{2}}^{d, d+*}(\mathbb{Z} / 2, \mathbb{Z} / 2)
$$

which is related to the geometrical transfer $t r_{d}: \pi_{*}^{\mathbb{S}}\left(B(\mathbb{Z} / 2)_{+}^{\times d}\right) \rightarrow \pi_{*}^{\mathbb{S}}\left(\mathbb{S}^{0}\right)$ of the stable homotopy of spheres. More explicitly, $t r_{d}$ induces $T r_{d}$ at the $E^{2}$-term of the Adams spectral sequence [1]. These transfers play a key role in the study of the Kervaire invariant one problem, which is one of the oldest unresolved issues in Differential and Algebraic topology. This problem was first introduced by Browder [8] where he indicated that smooth framed manifolds of Kervaire invariant one exist only in dimensions of the form $2^{i+1}-2$, and that a manifold exists in that dimension if and only if the class $h_{i}^{2} \in \operatorname{Ext}_{\mathcal{A}_{2}}^{2,2^{i+1}}(\mathbb{Z} / 2, \mathbb{Z} / 2)$ in the $E^{2}$-term of the classical Adams spectral represents an element $\theta_{i}: \mathbb{S}^{2^{i+1}-2} \rightarrow \mathbb{S}^{0}$ in the stable homotopy groups of spheres $\pi_{2^{i+1}-2}^{\mathbb{S}}$. These elements $\theta_{i}$ for $0 \leq i \leq 5$ are known to exist (see also Lin-Mahowald [22]), but they do not exist when $i \geq 7$ (see the work of Hill, Hopkins, Ravenel [16] and the discussion therein). So far the case $i=6$ is not yet known.

Singer [57] points out the non-trivial value of $T r_{d}$ by proving that it is an isomorphism for $d \leq 2$. In 1993, by using a basis consisting of the all the classes represented by certain polynomials in $\mathscr{P}_{3}$, Boardman indicated in [6] that $\operatorname{Tr}_{3}$ is also an isomorphism. Through these events, the Singer cohomological transfer can be viewed as an useful tool in the study of the $d$-th cohomology groups of the Steenrod algebra. Many mathematicians then investigated this transfer map (see Bruner et. al. [9], Chơn and Hà [11], Crossley [14], Hà [15], Hưng [19], Minami [26], Nam [31], the present author [41, 42, 43, 45, 46, 47, 48, 49, 50, 51, 52], Sum [63, 64, 65, 67] and others). In [57], using the invariant theory, Singer claims that $T r_{d}$ is an isomorphism for $d=4$ in a range of internal degrees, but $\operatorname{Tr}_{5}$ is not an epimorphism. Afterwards, he gave a hypothesis that the $\operatorname{transfer} \operatorname{Tr}_{d}$ 
is a monomorphism for any positive integer $d$. This prediction is currently still open for arbitrary $d>4$.

For a non-negative integer $n$, let $\left(\mathscr{P}_{d}\right)_{n}$ be the subspace of $\mathscr{P}_{d}$ consisting of all the homogeneous polynomials of degree $n$ in $\mathscr{P}_{d}$. Denote by $\left(Q \mathscr{P}_{d}\right)_{n}$ the subspace of $Q \mathscr{P}_{d}$ consisting of all the classes represented by the homogeneous polynomials in $\left(\mathscr{P}_{d}\right)_{n}$. One of the extremely useful tools for computing the hit problem and studying Singer's transfer is the Kameko squaring operation [21] $\left(\widetilde{S q_{*}^{0}}\right)_{(d, 2 n+d)}:\left(Q \mathscr{P}_{d}\right)_{2 n+d} \rightarrow\left(Q \mathscr{P}_{d}\right)_{n}$, which is an epimorphism of $\mathbb{Z} / 2\left(G L_{d}\right)$-modules. We refer to Sect.2 for its precise meaning. Let $\mu(n)$ denote the smallest number $u$ such that $\alpha(n+u) \leq u$, where $\alpha(k)$ is the number of 1's in the dyadic expansion of the positive integer $k$. By Kameko [21], if $\mu(2 n+d)=d$, then $\left(\widetilde{S q_{*}^{0}}\right)_{(d, 2 n+d)}$ is an $\mathbb{Z} / 2\left(G L_{d}\right)$-module isomorphism.

Recall that to solve the hit problem of Peterson, we will determine $Q \mathscr{P}_{d}$ in each degree $n \geq 1$. However, as explicitly shown in [41], it is enough to consider this space in the following "generic degree":

$$
n=r\left(2^{t}-1\right)+2^{t} s,
$$

whenever $r, t, s$ are non-negative integers such that $0 \leq \mu(s)<r \leq d$. Hit problems have been completely solved in $[21,37,61,62]$ for $d \leq 4$. For $r=d-1$ and $s>0$, it was investigated by Crabb-Hubbuck [12], Nam [30], Repka-Selick [54], and Sum [62]. For $r=d-1$ and $s=0$, it is partially studied by Mothebe [27] and by us [39,40]. The case $r=d-2=3$ was probed by the present author [41] for $s=1$, and by Sum [66] for $s=2^{m+u}+2^{m}-2, m \geq 0, u>0, t \geq 6$. The recent results when $r=d=5$ were explicitly determined in $[65,67,69]$ for $s \in\{2,3,5,7,10\}$, and by the present author [42] for $s=6$. The case $r=d=5, s=26$ and $t=0$ was studied by WalkerWood [71]. The authors indicated in [71] that in any minimal generating set for the $\mathcal{A}_{2}$-module $\mathscr{P}_{d}$, there are $2^{\left(\begin{array}{c}d \\ 2\end{array}\right)}$ elements in degree $2^{d}-d-1$. For $d=5$, we see that $2^{5}-6=26=5\left(2^{0}-1\right)+26.2^{0}$ and $\operatorname{dim}\left(\left(Q \mathscr{P}_{5}\right)_{\left.5\left(2^{0}-1\right)+26.2^{0}\right)}\right) 2^{\left(\begin{array}{l}5 \\ 2\end{array}\right)}=1024$. More generally, in generic degree of form $(1.1)$ for $d=r=5, s=26$ and $t \geq 0$, we have $\mu\left(5\left(2^{t}-1\right)+26.2^{t}\right)=5$ for all $t>0$. This implies that the iterated Kameko map $\left(\left(\widetilde{S q_{*}^{0}}\right)_{\left(5,5\left(2^{t}-1\right)+26.2^{t}\right)}\right)^{t}:\left(Q \mathscr{P}_{5}\right)_{5\left(2^{t}-1\right)+26.2^{t}} \rightarrow\left(Q \mathscr{P}_{5}\right)_{26}$ is an isomorphism for all $t \geq 0$. So, $Q \mathscr{P}_{5}$ has dimension 1024 in degree $5\left(2^{t}-1\right)+26.2^{t}$ for any $t \geq 0$. This event and the result for the case $d=6, t \geq 5$ have also been studied in [70]. For $d=r=5, s=42, t \geq 0$, and $d=6, r=5, s=42, t \geq 5$, we remark that since $\mu\left(5\left(2^{t}-1\right)+42.2^{t}\right)=5$ for $t>0$, the iterated homomorphism $\left(\left(\widetilde{S q_{*}^{0}}\right)_{\left(5,5\left(2^{t}-1\right)+42.2^{t}\right)}\right)^{t}:\left(Q \mathscr{P}_{5}\right)_{5\left(2^{t}-1\right)+42.2^{t}} \rightarrow\left(Q \mathscr{P}_{5}\right)_{42}$ is an isomorphism for arbitrary $t \geq 0$. By using a computer program of Robert R. Bruner written in MAGMA, we get $\operatorname{dim}\left(Q \mathscr{P}_{5}\right)_{5\left(2^{t}-1\right)+42.2^{t}}=2520$ for all $t \geq 0$. The direct proofs of this result will be published in detail elsewhere. On the other hand, notice that $\mu(42)=4$ and $\alpha(42+\mu(42))=4=\mu(42)$, by Sum [62, Theorem 1.3], it may be concluded that

$$
\operatorname{dim}\left(\left(Q \mathscr{P}_{6}\right)_{5\left(2^{t}-1\right)+42.2^{t}}\right)=\left(2^{6}-1\right) \operatorname{dim}\left(\left(Q \mathscr{P}_{5}\right)_{42}\right)=158760 \text { for all } t \geq 5 .
$$

It is currently difficult to solve hit problems in the general case.

In the present work, based on our works in [39, 40,41,42], we continue our study of the hit problem of five variables in generic degree of (1.1). At the same time, by using these computational techniques, we examine Singer's algebraic transfer of rank 5 in some internal degrees. More precisely, we explicitly determine a basis of $Q \mathscr{P}_{d}$ for $d=5$ and generic degree of (1.1) with $r=d=5, s=8$ and $t$ an arbitrary non-negative integer. (A basis of this space is a set consisting of all the classes represent by the admissible monomials of degree $n$ in $\mathscr{P}_{d}$. We refer to Sect.2 for the concept of the admissible monomial.) Using this result combining with the computations of $\operatorname{Ext}_{\mathcal{A}_{2}}^{5,13.2^{t}}(\mathbb{Z} / 2, \mathbb{Z} / 2$ ) (see Tangora [68], Chen [10], Lin [23]), and a direct computation using a result in [11] on the representation in the $\mathbb{Z} / 2$-lambda algebra $\Lambda$ of the transfer homomorphism of rank 5 , we show that $\operatorname{Tr}_{5}$ is an isomorphism when acting on $\mathbb{Z} / 2 \otimes_{G L_{5}} P_{\mathcal{A}_{2}} H_{13.2^{t}-5}\left(B(\mathbb{Z} / 2)^{\times 5}\right)$ for $t \in\{0,1\}$. (The information on the algebra $\Lambda$ can be found below in this section.) This gives a quite efficient method to study the isomorphism of the fifth transfer in some internal degrees of (1.1). Furthermore, our approach is different from that of Boardman [6], and of Singer [57]. The following is our first main result.

Theorem 1.1. Let us consider the generic degree $13.2^{t}-5$ with $t$ an arbitrary non-negative integer. Then, we have

$$
\operatorname{dim}\left(Q \mathscr{P}_{5}\right)_{13.2^{t}-5}= \begin{cases}174 & \text { if } t=0 \\ 840 & \text { if } t=1 \\ 1894 & \text { if } t \geq 2\end{cases}
$$

Note that $13.2^{t}-5=5\left(2^{t}-1\right)+8.2^{t}$. The theorem will be proved by determining explicitly an admissible monomial basis for $Q \mathscr{P}_{5}$ in generic degree $13.2^{t}-5$. The first idea for the proof of the theorem is from Kameko's squaring operation. More clearly, since

$$
5\left(2^{t}-1\right)+8.2^{t}=2^{t+3}+2^{t+2}+2^{t-1}+2^{t-2}+2^{t-2}-5,
$$


$\mu\left(13.2^{t}-5\right)=5$ for all $t>2$, which leads to the iterated linear transformation

$$
\left(\widetilde{S q_{*}^{0}}\right)_{\left(5,13.2^{t}-5\right)}^{t-2}:\left(Q \mathscr{P}_{5}\right)_{13.2^{t-5}} \rightarrow\left(Q \mathscr{P}_{5}\right)_{13.2^{2}-5}
$$

being an isomorphism of $\mathbb{Z} / 2\left(G L_{5}\right)$-modules for any $t \geq 2$ and therefore we need only to study the structure of $\left(Q \mathscr{P}_{5}\right)_{13.2^{t}-5}$ for $0 \leq t \leq 2$. The result when $t=0$ was computed by Tín [70]. We remark that for $t \in\{1,2\}$, since $\left(\widetilde{S q_{*}^{0}}\right)_{\left(5,13.2^{t}-5\right)}:\left(Q \mathscr{P}_{5}\right)_{13.2^{t}-5} \rightarrow\left(Q \mathscr{P}_{5}\right)_{13.2^{t-1}-5}$ is an epimorphism, we need only to determine the kernel of $\left(\widetilde{S q_{*}^{0}}\right)_{\left(5,13.2^{t}-5\right)}$. To study this space, we combine our recent results in [39] with previous results by Kameko [21], Mothebe [28, 29], Singer [58], Sum [62], and Tín [70].

Recently, Sum [65] has proved some properties of $\mathcal{A}_{2}$-generators for $\mathscr{P}_{d}$. Then, he made a conjecture on the relation between the admissible monomials for the polynomial algebras (see Sect.3). The conjecture helps us to reduce remarkably in computing the hit problem. From the results of Peterson [37], Kameko [21] and Sum [62], this conjecture holds true for $d \leq 4$. Sum proved in [65] that the conjecture is true in the case $d=5$ and the degree $n$ of the form (1.1) for $(r ; s)=(5 ; 10)$ and $t \geq 0$. Based upon the proof of Theorem 1.1, and previous results of the present author and Sum (see [39, 40,41, 42, 66]), the conjecture also satisfies for $d=5$ and in degrees of the form $(1.1)$ for $(r ; s)=(4 ; 0),(5 ; 6),(5 ; 8)$ and $(3 ; s)$, for $s=1, t \geq 0$, and $s=2^{m+u}+2^{m}-2, m \geq 0, u>0, t \geq 6$.

It is well known that $\mathbb{Z} / 2 \otimes_{G L_{d}} P_{\mathcal{A}_{2}} H_{n}\left(B(\mathbb{Z} / 2)^{\times d}\right)$ is dual to $\left(Q \mathscr{P}_{d}\right)_{n}^{G L_{d}}$, the subspace of $Q \mathscr{P}_{d}$ generated all $G L_{d}$-invariants of degree $n$. Computation of the $G L_{d}$-invariants is very difficult, particularly for values of $d$ as large as $d=5$. The understanding of special cases should be a helpful step toward the solution of the general problem. Now, applying Theorem 1.1 for $t=1$, we get the following, which is our second main result.

Theorem 1.2. There exists uniquely a non-zero class in $\left(Q \mathscr{P}_{5}\right)_{13.2^{1}-5}$ invariant under the usual action of $G L_{5}$. This implies that $\left(Q \mathscr{P}_{5}\right)_{13.2^{1-5}}^{G L_{5}}$ is one-dimensional.

One should note that Kameko's map $\left(\widetilde{S q_{*}^{0}}\right)_{\left(5,13.2^{1}-5\right)}:\left(Q \mathscr{P}_{5}\right)_{13.2^{1}-5} \rightarrow\left(Q \mathscr{P}_{5}\right)_{13.2^{0}-5}$ is an epimorphism of $\mathbb{Z} / 2\left(G L_{5}\right)$-modules. So, in order to prove Theorem 1.2 , we describe the $\mathbb{Z} / 2$-vector space structure of $\left(Q \mathscr{P}_{5}\right)_{13.2^{0}-5}^{G L_{5}}$. Using this and a monomial basis of $\left(Q P_{5}\right)_{21}$ given in the proof of Theorem 1.1, we explicitly compute all $G L_{5}$-invariants of $Q \mathscr{P}_{5}$ in degree $13.2^{1}-5$.

In addition to the Singer transfer mentioned above, the $(\bmod 2)$ lambda algebra $\Lambda$ (see Bousfield et al. [7]) is also one of the important tools to study mod-2 cohomology groups $\operatorname{Ext}_{\mathcal{A}_{2}}^{d, d+*}(\mathbb{Z} / 2, \mathbb{Z} / 2)$. $\Lambda$ is defined as a differential, bigraded, associative algebra with unit over $\mathbb{Z} / 2$, is generated by $\lambda_{i} \in \Lambda^{1, i}$, satisfying the Adem relations

$$
\lambda_{i} \lambda_{2 i+d+1}=\sum_{j \geq 0}\left(\begin{array}{c}
d-j-1 \\
j
\end{array}\right) \lambda_{i+d-j} \lambda_{2 i+1+j} \quad(i \geq 0, d \geq 0)
$$

and the differential

$$
\partial\left(\lambda_{d-1}\right)=\sum_{j \geq 1}\left(\begin{array}{c}
d-j-1 \\
j
\end{array}\right) \lambda_{d-j-1} \lambda_{j-1} \quad(d \geq 1),
$$

where $\left(\begin{array}{c}d-j-1 \\ j\end{array}\right)$ denotes the binomial coefficient reduced modulo 2. Furthermore, we have

$$
H^{d, *}(\Lambda, \partial)=\operatorname{Ext}_{\mathcal{A}_{2}}^{d, d+*}(\mathbb{Z} / 2, \mathbb{Z} / 2) .
$$

For non-negative integers $j_{1}, \ldots, j_{d}$, a monomial $\lambda_{j_{1}} \ldots \lambda_{j_{d}} \in \Lambda$ is called the monomial of the length $d$. We shall write $\lambda_{J}, J=\left(j_{1}, \ldots, j_{d}\right)$ for $\prod_{1 \leq k \leq d} \lambda_{j_{k}}$ and refer to $\ell(J)=d$ as the length of $J$. It should be noted that the algebra $\Lambda$ is not commutative and that the bigrading of a monomial indexed by $J$ may be written $(d, n)$, where the homological degree $d$, as above, is the length of $J$, and $n=\sum_{1 \leq k \leq d} j_{k}$. A monomial $\lambda_{J}$ is called admissible if $j_{k} \leq 2 j_{k+1}$ for all $1 \leq k \leq d-1$. By the relations (1.2), the $\mathbb{Z} / 2$-vector subspace

$$
\Lambda^{d, *}=\left\langle\left\{\lambda_{J} \mid J=\left(j_{1}, \ldots, j_{d}\right), j_{k} \geq 0,1 \leq k \leq d\right\}\right\rangle
$$

of $\Lambda$ has an additive basis consisting of all admissible monomials of the length $d$. Recall that the dual of $\mathscr{P}_{d}$ is isomorphic to $\Gamma\left(a_{1}, \ldots, a_{d}\right)$, the divided power algebra generated by $a_{1}, \ldots, a_{d}$, where $a_{t}=a_{t}^{(1)}$ is dual to $x_{t}$ with respect to the basis of $\mathscr{P}_{d}$ consisting of all monomials in $x_{1}, \ldots, x_{d}$. In other words, $H_{*}\left(B(\mathbb{Z} / 2)^{\times d}\right)=H_{*}\left((\mathbb{Z} / 2)^{\times d}\right) \cong \Gamma\left(a_{1}, \ldots, a_{d}\right)$. We note that the algebra $H_{*}\left(B(\mathbb{Z} / 2)^{\times d}\right)$ has a right $\mathcal{A}_{2}$-module structure. The right action of $\mathcal{A}_{2}$ on this algebra is given by $\left(a_{t}^{(j)}\right) S q^{k}=\left(\begin{array}{c}j-k \\ k\end{array}\right) a_{t}^{(j-k)}=S q_{*}^{k}\left(a_{t}^{(j)}\right)$ and Cartan's formula. (Note that $S q_{*}^{k}$ denotes the dual of $S q^{k}$.) In [11], Chơn and Hà have established a homomorphism $\psi_{d}: H_{*}\left(B(\mathbb{Z} / 2)^{\times d}\right) \longrightarrow \Lambda^{d, *}$, 
which is considered as a representation in the $\mathbb{Z} / 2$-lambda algebra of Singer's transfer of rank $d$ and determined by the following inductive formula:

$$
\psi_{d}\left(a^{J}\right)= \begin{cases}\lambda_{j_{1}} & \text { if } \ell(J)=1, \\ \sum_{t \geq j_{d}} \psi_{d-1}\left(\prod_{1 \leq k \leq d-1} a_{k}^{\left(j_{k}\right)} S q^{t-j_{d}}\right) \lambda_{t} & \text { if } \ell(J)>1,\end{cases}
$$

for any $a^{J}:=\prod_{1 \leq k \leq d} a_{k}^{\left(j_{k}\right)} \in H_{*}\left(B(\mathbb{Z} / 2)^{\times d}\right)$ and $J:=\left(j_{1}, j_{2}, \ldots, j_{d}\right)$. Note that $\psi_{d}$ is not an $\mathcal{A}_{2}$-homomorphism. The authors showed in [11] that if $Z \in P_{\mathcal{A}_{2}} H_{*}\left(B(\mathbb{Z} / 2)^{\times d}\right)$, then $\psi_{d}(Z)$ is a cycle in $\Lambda^{d, *}$ and $\operatorname{Tr}_{d}([Z])=\left[\psi_{d}(Z)\right]$. Applying this event and Theorem 1.2 into the investigation of the Singer transfer of rank 5, we obtain the following theorem, which is our third main result.

Theorem 1.3. The cohomological transfer

$$
\operatorname{Tr}_{5}: \mathbb{Z} / 2 \otimes_{G L_{5}} P_{\mathcal{A}_{2}} H_{13.2^{1}-5}\left(B(\mathbb{Z} / 2)^{\times 5}\right) \rightarrow \operatorname{Ext}_{\mathcal{A}_{2}}^{5+5+\left(13.2^{1}-5\right)}(\mathbb{Z} / 2, \mathbb{Z} / 2)
$$

is an isomorphism.

As it is known, there exists an endomorphism $S q^{0}$ of the lambda algebra $\Lambda$, determined by $S q^{0}\left(\lambda_{J}=\prod_{1 \leq k \leq d} \lambda_{j_{k}}\right)=\prod_{1 \leq k \leq d} \lambda_{2 j_{k}+1}$, where $\lambda_{J}$ is not necessarily admissible. It respects the relations in (1.2) and commutes with the differential $\partial$ in (1.3). Then, $S q^{0}$ induces the classical squaring operation in the Ext groups

$$
S q^{0}: H^{d, *}(\Lambda, \partial)=\operatorname{Ext}_{\mathcal{A}_{2}}^{d, d+*}(\mathbb{Z} / 2, \mathbb{Z} / 2) \rightarrow H^{d, d+2 *}(\Lambda, \partial)=\operatorname{Ext}_{\mathcal{A}_{2}}^{d, 2(d+*)}(\mathbb{Z} / 2, \mathbb{Z} / 2) .
$$

This $S q^{0}$ is not the identity map (see [24]). As above mentioned, the structure of the groups $\operatorname{Ext}_{\mathcal{A}_{2}}^{d, d+*}(\mathbb{Z} / 2, \mathbb{Z} / 2)$ has been intensively studied by many authors, but remains very mysterious in general. In what follows, $\left(S q^{0}\right)^{t}: \operatorname{Ext}_{\mathcal{A}_{2}}^{*, *}(\mathbb{Z} / 2, \mathbb{Z} / 2) \rightarrow \operatorname{Ext}_{\mathcal{A}_{2}}^{*, *}(\mathbb{Z} / 2, \mathbb{Z} / 2)$ denotes the composite $S q^{0} \ldots S q^{0}$ ( $t$ times of $\left.S q^{0}\right)$ if $t>1$, is $S q^{0}$ if $t=1$, and is the identity map if $t=0$. A family $\left\{a_{t}: t \geq 0\right\} \subset \operatorname{Ext}_{\mathcal{A}_{2}}^{d, d+*}(\mathbb{Z} / 2, \mathbb{Z} / 2)$ is called a $S q^{0}$-family if $a_{t}=\left(S q^{0}\right)^{t}\left(a_{0}\right)$ for $t \geq 0$. We now return to the internal degree $13.2^{t}-5$ in Theorem 1.1. It has been shown (see Tangora [68], Lin [23], Chen [10]) that

$$
\operatorname{Ext}_{\mathcal{A}_{2}}^{5,5+\left(13.2^{t}-5\right)}(\mathbb{Z} / 2, \mathbb{Z} / 2)= \begin{cases}0 & \text { if } t=0, \\ \left\langle h_{t+1} f_{t-1}\right\rangle & \text { if } t \geq 1,\end{cases}
$$

and that $h_{t+1} f_{t-1}=h_{t} g_{t} \neq 0$, where $h_{t}=\left(S q^{0}\right)^{t}\left(h_{0}\right)$ is the Adams element in $\operatorname{Ext}_{\mathcal{A}_{2}}^{1,2^{t}}(\mathbb{Z} / 2, \mathbb{Z} / 2)$, $g_{t}=\left(S q^{0}\right)^{t-1}\left(g_{1}\right)$ and $f_{t-1}=\left(S q^{0}\right)^{t-1}\left(f_{0}\right)$ are the elements non-zero in $\operatorname{Ext}_{\mathcal{A}_{2}}^{4,12.2^{t}}(\mathbb{Z} / 2, \mathbb{Z} / 2)$ and $\operatorname{Ext}_{\mathcal{A}_{2}}^{4,11.2^{t}}(\mathbb{Z} / 2, \mathbb{Z} / 2)$, respectively, for any $t \geq 1$. (Note that by Lin $[23]$, the groups $\operatorname{Ext}_{\mathcal{A}_{2}}^{4,4+*}(\mathbb{Z} / 2, \mathbb{Z} / 2)$ contains seven $S q^{0}$-families of indecomposable elements, namely

$$
\left.\left\{d_{t}\right\},\left\{e_{t}\right\},\left\{f_{t}\right\},\left\{g_{t+1}\right\},\left\{p_{t}\right\},\left\{D_{3}(t)\right\},\left\{p_{t}^{\prime}\right\}(t \geq 0)\right) .
$$

As well known, Singer [57] showed that the transfer $\operatorname{Tr}_{1}$ detects the family $\left\{h_{t} \mid t \geq 1\right\}$ and that $\bigoplus_{d>0} \operatorname{Tr}_{d}$ is an algebra homomorphism. Following Nam [31], the family $\left\{f_{t-1} \mid t \geq 1\right\}$ was detected by $\bar{T} r_{4}$. These data imply that $h_{t+1} f_{t-1}$ is in the image of $T r_{5}$ for all $t \geq 1$. In Sect.5, we give another direct proof of this event for the case $t=1$. More specifically, we proved $h_{2} f_{0} \in \operatorname{Im}\left(\operatorname{Tr}_{5}\right)$ by using Theorem 1.2 and a representation of $\operatorname{Tr}_{5}$ over the lambda algebra.

As above shown, to prove Theorem 1.2, we need to determine all $G L_{5}$-invariants of $\left(Q \mathscr{P}_{5}\right)_{13.2^{0}-5}$. Applying Theorem 1.1 for $t=0$ with a basis of $Q \mathscr{P}_{5}$ in degree $13.2^{0}-5$ (see [70]), we showed that $\left(Q \mathscr{P}_{5}\right)_{13.2^{0}-5}^{G L_{5}}$ is zero (see Theorem 4.1.1 in Sect.4). This result together with a fact of the fifth cohomology group $\operatorname{Ext}_{\mathcal{A}_{2}}^{5,5+\left(13.2^{0}-5\right)}(\mathbb{Z} / 2, \mathbb{Z} / 2)$, it may be concluded that $\operatorname{Tr}_{5}$ is a trivial isomorphism when acting on the space $\mathbb{Z} / 2 \otimes_{G L_{5}} P_{\mathcal{A}_{2}} H_{13.2^{0}-5}\left(B(\mathbb{Z} / 2)^{\times 5}\right)$. As an immediate consequence from this and Theorem 1.3, we get

Corollary 1.4. Singer's conjecture for $\operatorname{Tr}_{5}$ holds in the bidegrees $(5,5+8)$ and $(5,5+21)$.

To end this introduction, we will discuss whether $T r_{5}$ is an isomorphism or not in the bidegree $\left(5,5+\left(13.2^{t}-5\right)\right)$ for $t \geq 2$. Since the iterated Kameko homomorphism

$$
\left(\left(\widetilde{S q_{*}^{0}}\right)_{\left(5,13.2^{t}-5\right)}\right)^{t-2}:\left(Q \mathscr{P}_{5}\right)_{13.2^{t}-5} \rightarrow\left(Q \mathscr{P}_{5}\right)_{13.2^{2}-5}
$$

is an $G L_{5}$-module isomorphism for all $t \geq 2$, to examine Singer's conjecture for $\operatorname{Tr}_{5}$ in the above bidegree, we need only to determine all $G L_{5}$-invariants of $\left(Q \mathscr{P}_{5}\right)_{13.2^{t}-5}$ for $t=2$. Recall that Kameko's map

$$
\left(\widetilde{S q_{*}^{0}}\right)_{\left(5,13.2^{2}-5\right)}:\left(Q \mathscr{P}_{5}\right)_{13.2^{2}-5} \rightarrow\left(Q \mathscr{P}_{5}\right)_{13.2^{1}-5}
$$

is an epimorphism of $G L_{5}$-modules and that the element $h_{3} f_{1} \in \operatorname{Ext}_{\mathcal{A}_{2}}^{5,5+\left(13.2^{2}-5\right)}(\mathbb{Z} / 2, \mathbb{Z} / 2)$ is in the image of $\operatorname{Tr}_{5}$. So, by Theorem 1.2, we deduce

$$
1 \leq \operatorname{dim}\left(\mathbb{Z} / 2 \otimes_{G L_{5}} P_{\mathcal{A}_{2}} H_{13.2^{2}-5}\left(B(\mathbb{Z} / 2)^{\times 5}\right)\right) \leq \operatorname{dim}\left(\operatorname{Ker}\left(\widetilde{S q_{*}^{0}}\right)_{\left(5,13.2^{2}-5\right)}\right)^{G L_{5}}+1 .
$$


Furthermore, all elements of $\mathbb{Z} / 2 \otimes_{G L_{5}} P_{\mathcal{A}_{2}} H_{13.2^{2}-5}\left(B(\mathbb{Z} / 2)^{\times 5}\right)$ are of the form

$$
\left(\gamma\left[\varphi\left(u_{0}\right)\right]+[v]\right)^{*}
$$

where $\gamma \in \mathbb{Z} / 2$, and the mapping $\varphi: \mathscr{P}_{5} \rightarrow \mathscr{P}_{5}$ determined by setting $\varphi(u)=x_{1} x_{2} x_{3} x_{4} x_{5} u^{2}$ for any $u \in \mathscr{P}_{5}, v \in\left(\mathscr{P}_{5}\right)_{13.2^{2}-5}$ such that $[v]$ belongs to $\operatorname{Ker}\left(\widetilde{S q_{*}^{0}}\right)_{\left(5,13.2^{2}-5\right)}$, and $u_{0} \in\left(\mathscr{P}_{5}\right)_{13.2^{1}-5}$. Based on Theorem 1.2, $\left[u_{0}\right]$ is the only non-zero element in $\left(Q \mathscr{P}_{5}\right)_{13.2^{1}-5}^{G L_{5}}$. Direct calculating the elements $\left(\gamma\left[\varphi\left(u_{0}\right)\right]+[v]\right)^{*}$ is a hard work. By using techniques of the hit problem of five variables, we will describe explicitly all these elements in the near future. From these data with the fact that $h_{t+1} f_{t-1} \in \operatorname{Im}\left(T r_{5}\right)$ for $t \geq 1$, we conclude that if $\left(\operatorname{Ker}\left(\widetilde{S q_{*}^{0}}\right)_{\left(5,13.2^{2}-5\right)}\right)^{G L_{5}}$ is zero, then $\operatorname{Tr}_{5}$ is an isomorphism in the bidegree $\left(5,5+\left(13.2^{t}-5\right)\right)$ for every $t \geq 2$. This means that Singer's conjecture for $\operatorname{Tr}_{5}$ also satisfies in this bidegree. However, it will be much more interesting if $\left(\operatorname{Ker}\left(\widetilde{S q_{*}^{0}}\right)_{\left(5,13.2^{2}-5\right)}\right)^{G L_{5}}$ is non-trivial and $\operatorname{dim}\left(Q \mathscr{P}_{5}\right)_{13.2^{2}-5}^{G L_{5}} \neq 1$.

The structure of the paper is as follows. First, some background is reviewed in Sect.2. In the next section, we present Singer's criterion on $\mathcal{A}_{2}$-decomposable and Sum's conjecture related to the minimal set of generators for $\mathcal{A}_{2}$-modules $\mathscr{P}_{d}$. Then, the $\mathcal{A}_{2}$-generators for $\mathscr{P}_{5}$ in degree $13.2^{t}-5$ are described explicitly by proving Theorem 1.1. In Sect.4, we prove Theorem 1.2 by using the admissible monomial bases of $\left(Q \mathscr{P}_{5}\right)_{13.2^{0}-5}$ and $\left(Q \mathscr{P}_{5}\right)_{13.2^{1}-5}$. Based upon Theorem 1.2 and a representation in the lambda algebra of the fifth Singer transfer, the proof of Theorem 1.3 is presented in Sect.5. All the admissible monomials of degree $13.2^{t}-5$ in $\mathscr{P}_{5}$ are provided in Sect.6 of the online version [44].

Acknowledgments. This research is supported financially by the National Foundation for Science and Technology Development (NAFOSTED) of Viet Nam under Grant No. 101.04-2017.05.

The author is very grateful to the anonymous referees for the careful reading of the manuscript and their insightful comments and detailed suggestions, which have led me to improve considerably this work.

Finally, I would also like to thank Robert Bruner, Phan Hoàng Chơn, Mbakiso Fix Mothebe for illuminating conversations on the hit problem and the Ext groups, Hadi Zare for sending a copy of [78], and Lê Minh Hà for pointing out an error in the original manuscript.

\section{Preliminaries}

This section starts with a recollection of the Kameko squaring operation and some information related to the Peterson hit problem.

\subsection{Kameko's squaring operation}

Recall that the polynomial algebra $\mathscr{P}_{d}=\mathbb{Z} / 2\left[x_{1}, \ldots, x_{d}\right]$ is an unstable left module on the ring $\mathcal{A}_{2}$. Let $G L_{d}:=G L(d, \mathbb{Z} / 2)$ denote the general linear group of rank $d$ over the field $\mathbb{Z} / 2$. An usual right action of this group on $\mathscr{P}_{d}$ is given by $((f) w)\left(x_{1}, x_{2}, \ldots, x_{d}\right)=f\left(\left(x_{1}\right) w,\left(x_{2}\right) w, \ldots,\left(x_{d}\right) w\right)$, where $w=\left(w_{i j}\right) \in G L_{d}$ and $\left(x_{j}\right) w=\sum_{1<i<d} x_{i} w_{i j}, 1 \leq j \leq d$. Thus, $\mathscr{P}_{d}$ (resp. $\left.\left(\mathscr{P}_{d}\right)^{*}\right)$ has also a right (resp. left) $G L_{d}$-module structure. Furthermore, since the two actions of $\mathcal{A}_{2}$ and $G L_{d}$ upon $\mathscr{P}_{d}$ (resp. $\left.\left(\mathscr{P}_{d}\right)^{*}\right)$ commute with each other, there is an inherited action of $G L_{d}$ on $Q \mathscr{P}_{d}$ (resp. $\left.\left(Q \mathscr{P}_{d}\right)^{*}=P_{\mathcal{A}_{2}} H_{*}\left(B(\mathbb{Z} / 2)^{\times d}\right)\right)$.

We knew that the homological algebra $\left\{H_{n}\left(B(\mathbb{Z} / 2)^{\times d}\right) \mid n \geq 0\right\}$ is dual to $\mathscr{P}_{d}$. Moreover, it is isomorphic to $\Gamma\left(a_{1}, \ldots, a_{d}\right)$, the divided power algebra generated by $a_{1}, \ldots, a_{d}$, each of degree one, where $a_{j}=a_{j}^{(1)}$ is dual to $x_{j}$. Here the duality is taken with respect to the basis of $\mathscr{P}_{d}$ consisting of all monomials in $x_{1}, \ldots, x_{d}$. We now denote by $P_{\mathcal{A}_{2}} H_{n}\left(B(\mathbb{Z} / 2)^{\times d}\right)$ the primitive subspace consisting of all elements in $H_{n}\left(B(\mathbb{Z} / 2)^{\times d}\right)$, which are annihilated by every Steenrod's operation $S q^{k}, k>0$. So, it is dual to $\left(Q \mathscr{P}_{d}\right)_{n}$. By Kameko [21], we have the monomorphism of $G L_{d}$-modules

$$
\overline{S q}^{0}: P_{\mathcal{A}_{2}} H_{n}\left(B(\mathbb{Z} / 2)^{\times d}\right) \longrightarrow P_{\mathcal{A}_{2}} H_{d+2 n}\left(B(\mathbb{Z} / 2)^{\times d}\right)
$$

which induces the following squaring operation in the dual of the spaces $\left(Q \mathscr{P}_{d}\right)_{*}^{G L_{d}}$ :

$$
\widetilde{S q^{0}}=i d_{\mathbb{Z} / 2} \otimes_{G L_{d}} \overline{S q}^{0}: \mathbb{Z} / 2 \otimes_{G L_{d}} P_{\mathcal{A}_{2}} H_{n}\left(B(\mathbb{Z} / 2)^{\times d}\right) \rightarrow \mathbb{Z} / 2 \otimes_{G L_{d}} P_{\mathcal{A}_{2}} H_{d+2 n}\left(B(\mathbb{Z} / 2)^{\times d}\right) .
$$

This $\widetilde{S q^{0}}$ commutes with the classical squaring operation

$$
S q^{0}: \operatorname{Ext}_{\mathcal{A}_{2}}^{d, d+n}(\mathbb{Z} / 2, \mathbb{Z} / 2) \rightarrow \operatorname{Ext}_{\mathcal{A}_{2}}^{d, 2 d+2 n}(\mathbb{Z} / 2, \mathbb{Z} / 2)
$$


through the $d$-th Singer transfer (see [4], [6], [26]). In other words, the following diagram is commutative:

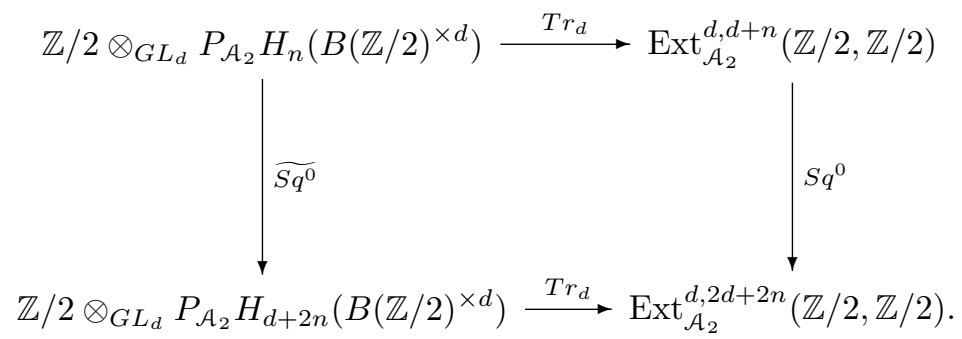

The dual homomorphism $\widetilde{S q_{*}^{0}}:\left(Q \mathscr{P}_{d}\right)_{d+2 n}^{G L_{d}} \rightarrow\left(Q \mathscr{P}_{d}\right)_{n}^{G L_{d}}$ of $\widetilde{S q^{0}}$ is induced by the homomorphism $\left(\widetilde{S q_{*}^{0}}\right)_{(d, d+2 n)}:\left(Q \mathscr{P}_{d}\right)_{d+2 n} \rightarrow\left(Q \mathscr{P}_{d}\right)_{n}$. The latter is given by the $\mathbb{Z} / 2$-linear map

$$
\begin{aligned}
\delta:\left(\mathscr{P}_{d}\right)_{d+2 n} & \longrightarrow\left(\mathscr{P}_{d}\right)_{n} \\
x_{1}^{t_{1}} x_{2}^{t_{2}} \ldots x_{d}^{t_{d}} & \longmapsto \begin{cases}x_{1}^{\frac{t_{1}-1}{2}} x_{2}^{\frac{t_{2}-1}{2}} \ldots x_{d}^{\frac{t_{d}-1}{2}} & \text { if } t_{1}, \ldots, t_{d} \text { odd }, \\
0 & \text { otherwise. }\end{cases}
\end{aligned}
$$

Denote by $\alpha(n)$ the number of 1's in dyadic expansion of $n$. We consider the arithmetic function (see [21], [67]):

$$
\begin{aligned}
\mu(n) & =\min \{u \in \mathbb{N}: \quad \alpha(n+u) \leq u\} \\
& =\min \left\{u \in \mathbb{N}: \quad n=\sum_{1 \leqslant j \leqslant u}\left(2^{s_{j}}-1\right), s_{j}>0,1 \leqslant j \leqslant u\right\} .
\end{aligned}
$$

From the above data, $\left(\widetilde{S q_{*}^{0}}\right)_{(d, d+2 n)}$ is an $\mathbb{Z} / 2\left(G L_{d}\right)$-module epimorphism. However, in particular, if $\mu(d+2 n)=d$ then it is an isomorphism. According to Hưng [19, Theorem 1.5], if Singer's conjecture for the $d$-th algebraic transfer is true, then $\operatorname{Tr}_{d}$ does not detect the non-zero elements $u \in \operatorname{Ext}_{\mathcal{A}_{2}}^{d, d+n}(\mathbb{Z} / 2, \mathbb{Z} / 2)$ such that $S q^{0}(u)=0$ and $\mu(2 n+d)=d$. In this case, $u$ is called critical. This leads us to the study of the kernel of $\widetilde{S q^{0}}$. Recall that $\overline{S q}^{0}$ is a monomorphism, but the squaring operation $\widetilde{S q^{0}}=i d_{\mathbb{Z} / 2} \otimes_{G L_{d}} \overline{S q}^{0}$ is not a monomorphism in general. Indeed, by using a computer calculation, Hưng provided a counter-example in [19] that $\widetilde{S q^{0}}$ is not a monomorphism when acting on $\mathbb{Z} / 2 \otimes_{G L_{5}} P_{\mathcal{A}_{2}} H_{15}\left(B(\mathbb{Z} / 2)^{\times 5}\right)$. This was confirmed again by the works of Sum $[63,67]$. Thereafter, Hưng [19] conjectured that $\widetilde{S q^{0}}$ is a monomorphism if and only if $d \leq 4$. By Boardman [6], and Singer [57], the conjecture satisfies for $d \leq 3$. We hope that it can be verified for $d=4$ by using the dual of $\widetilde{S q^{0}}$ and the results on the hit problem in [65].

Thus, to verify Singer's conjecture, in addition to the techniques of the hit problem mentioned in this paper, we can use the relationship between the algebraic transfer and critical elements. However, finding critical elements is not easy.

\subsection{On the classical "hit problem" of Peterson}

To study the hit problem, we need some relevant notations and concepts. For a natural number $n$, denote by $\alpha_{t}(n)$ the $t$-th coefficients in dyadic expansion of $n$. This means $\alpha(n)=\sum_{t \geq 0} \alpha_{t}(n)$. Further, $n$ can be represented as follows: $n=\sum_{t \geq 0} \alpha_{t}(n) 2^{t}$, where $\alpha_{t}(n) \in\{0,1\}, t=0,1, \ldots$ Consider the monomial $x=x_{1}^{u_{1}} x_{2}^{u_{2}} \ldots x_{d}^{u_{d}} \in \mathscr{P}_{d}$, we define two sequences associated with $x$ by $\omega(x):=\left(\sum_{1 \leq j \leq d} \alpha_{0}\left(u_{j}\right), \sum_{1 \leq j \leq d} \alpha_{1}\left(u_{j}\right), \ldots, \sum_{1 \leq j \leq d} \alpha_{t-1}\left(u_{j}\right), \ldots\right)$ and $\left(u_{1}, u_{2}, \ldots, u_{d}\right)$, which are called the weight vector and the exponent vector of $x$, respectively. From now on, we shall write $\omega_{t}(x)$ for $\sum_{1<j<d} \alpha_{t-1}\left(u_{j}\right), t=1,2, \ldots$

Let $\omega=\left(\omega_{1}, \omega_{2}, \ldots, \omega_{t}, \ldots\right)$ be a sequence of non-negative integers. Then, the sequence $\omega$ are called the weight vector if $\omega_{t}=0$ for $t \gg 0$. We define $\operatorname{deg}(\omega)=\sum_{t>1} 2^{t-1} \omega_{t}$. The sets of all the weight vectors and the exponent vectors are given the left lexicographical order.

Recall that a homogeneous element $f \in \mathscr{P}_{d}$ is called $\mathcal{A}_{2}$-decomposable (or "hit") if it is in the image of positive degree elements of $\mathcal{A}_{2}$. This means that $f$ belongs to $\mathcal{A}_{2}^{+} \mathscr{P}_{d}$.

The equivalence relations on $\mathscr{P}_{d}$ (see $\left.[21,41]\right)$. For a weight vector $\omega$, we denote two subspaces associated with $\omega$ :

$$
\begin{aligned}
& \mathscr{P}_{d}(\omega)=\left\langle\left\{x \in \mathscr{P}_{d} \mid \operatorname{deg}(x)=\operatorname{deg}(\omega), \omega(x) \leq \omega\right\}\right\rangle, \\
& \mathscr{P}_{d}^{-}(\omega)=\left\langle\left\{x \in \mathscr{P}_{d} \mid \operatorname{deg}(x)=\operatorname{deg}(\omega), \omega(x)<\omega\right\}\right\rangle .
\end{aligned}
$$

Let us now consider the homogeneous polynomials $f$, and $g$ in $\mathscr{P}_{d}$ with $\operatorname{deg}(f)=\operatorname{deg}(g)$. We define the following binary relations $" \equiv "$ and $" \equiv \equiv_{\omega}$ " on $\mathscr{P}_{d}$ :

(i) $f \equiv g$ if and only if $f=g$ modulo $\left(\mathcal{A}_{2}^{+} \mathscr{P}_{d}\right)$. Specifically, if $f \equiv 0$ then $f$ is $\mathcal{A}_{2}$-decomposable.

(ii) $f \equiv_{\omega} g$ if and only if $f, g \in \mathscr{P}_{d}(\omega)$ and $f=g$ modulo $\left(\left(\mathcal{A}_{2}^{+} \mathscr{P}_{d} \cap \mathscr{P}_{d}(\omega)\right)+\mathscr{P}_{d}^{-}(\omega)\right)$. 
It is easily seen that these binary relations are equivalence ones. Let $Q \mathscr{P}_{d}(\omega)$ denote the quotient of $\mathscr{P}_{d}(\omega)$ by the equivalence relation "三 $\omega$ ". Then, by the above definition and Sum's work [67], one has a $G L_{d}$-module $Q \mathscr{P}_{d}(\omega)=\mathscr{P}_{d}(\omega) /\left(\left(\mathcal{A}_{2}^{+} \mathscr{P}_{d} \cap \mathscr{P}_{d}(\omega)\right)+\mathscr{P}_{d}^{-}(\omega)\right)$, from which we have the following, which are shown in [41]; however, to make the paper self-contained, we would like to present again for reader's convenience.

$$
\begin{aligned}
\operatorname{dim}\left(\left(Q \mathscr{P}_{d}\right)_{n}\right) & =\sum_{\operatorname{deg} \omega=n} \operatorname{dim}\left(Q \mathscr{P}_{d}(\omega)\right), \\
\operatorname{dim}\left(\left(Q \mathscr{P}_{d}\right)_{n}^{G L_{d}}\right) & \leq \sum_{\operatorname{deg}(\omega)=n} \operatorname{dim}\left(Q \mathscr{P}_{d}(\omega)^{G L_{d}}\right) .
\end{aligned}
$$

Indeed, by Walker and Wood [73], we have a filtration of $Q \mathscr{P}_{d}$ :

$\{0\} \subseteq \cdots \subseteq \mathscr{P}_{d}^{-}(\omega) /\left(\left(\mathcal{A}_{2}^{+} \mathscr{P}_{d}\right) \cap \mathscr{P}_{d}^{-}(\omega)\right) \subseteq \mathscr{P}_{d}(\omega) /\left(\left(\mathcal{A}_{2}^{+} \mathscr{P}_{d}\right) \cap \mathscr{P}_{d}(\omega)\right) \subseteq \cdots \subseteq \mathscr{P}_{d} /\left(\mathcal{A}_{2}^{+} \mathscr{P}_{d}\right)=Q \mathscr{P}_{d}$

This is not only a filtration of $Q \mathscr{P}_{d}$ as a vector space, but also as a $G L_{d}$-module. The inclusion of $\mathscr{P}_{d}^{-}(\omega)$ into $\mathscr{P}_{d}(\omega)$ induces the monomorphism

$$
\mathscr{P}_{d}^{-}(\omega) /\left(\left(\mathcal{A}_{2}^{+} \mathscr{P}_{d}\right) \cap \mathscr{P}_{d}^{-}(\omega)\right) \rightarrow \mathscr{P}_{d}(\omega) /\left(\left(\mathcal{A}_{2}^{+} \mathscr{P}_{d}\right) \cap \mathscr{P}_{d}(\omega)\right)
$$

and the following sequence is short exact:

$$
\begin{aligned}
0 \rightarrow \mathscr{P}_{d}^{-}(\omega) /\left(\left(\mathcal{A}_{2}^{+} \mathscr{P}_{d}\right) \cap \mathscr{P}_{d}^{-}(\omega)\right) \rightarrow \mathscr{P}_{d}(\omega) /\left(\left(\mathcal{A}_{2}^{+} \mathscr{P}_{d}\right) \cap \mathscr{P}_{d}(\omega)\right) \rightarrow & \rightarrow \\
& \rightarrow \mathscr{P}_{d}(\omega) /\left(\left(\left(\mathcal{A}_{2}^{+} \mathscr{P}_{d}\right) \cap \mathscr{P}_{d}(\omega)\right)+\mathscr{P}_{d}^{-}(\omega)\right) \rightarrow 0 .
\end{aligned}
$$

So, one has an isomorphism of $\mathbb{Z} / 2 G L_{d}$-modules:

$$
Q \mathscr{P}_{d}(\omega) \cong\left(\mathscr{P}_{d}(\omega) /\left(\left(\mathcal{A}_{2}^{+} \mathscr{P}_{d}\right) \cap \mathscr{P}_{d}(\omega)\right)\right) /\left(\mathscr{P}_{d}^{-}(\omega) /\left(\left(\mathcal{A}_{2}^{+} \mathscr{P}_{d}\right) \cap \mathscr{P}_{d}^{-}(\omega)\right)\right) .
$$

These, together with the filtration of $Q \mathscr{P}_{d}$, imply the above claims.

The linear order on $\mathscr{P}_{d}($ see $[21])$. Let $u=x_{1}^{a_{1}} x_{2}^{a_{2}} \ldots x_{d}^{a_{d}}$ and $v=x_{1}^{b_{1}} x_{2}^{b_{2}} \ldots x_{d}^{b_{d}}$ be monomials of the same degree in $\mathscr{P}_{d}$. We write $a, b$ for the exponent vectors of $u, v$, respectively. We say that $a<b$ if there is a positive integer $m$ such that $a_{j}=b_{j}$ for all $j<m$ and $a_{m}<b_{m}$, and that $u<v$ if and only if one of the following holds:

(i) $\omega(u)<\omega(v)$;

(ii) $\omega(u)=\omega(v)$ and $a<b$.

The inadmissible monomial (see [21]). We say that a monomial $u \in \mathscr{P}_{d}$ is inadmissible, if there exist monomials $x_{1}, x_{2}, \ldots, x_{k}$ such that $x_{j}<u$ for $1 \leq j \leq k$ and $u \equiv \sum_{1<i<k} x_{i}$. Then, $u$ is said to be admissible, if it is not inadmissible.

It should be noticed that the set of all the admissible monomials of degree $n$ in $\mathscr{P}_{d}$ is a minimal set of $\mathcal{A}_{2}$-generators for $\mathscr{P}_{d}$ in degree $n$. So, $\left(Q \mathscr{P}_{d}\right)_{n}$ is an $\mathbb{Z} / 2$-vector space with a basis consisting of all the classes represented by the admissible monomials of degree $n$ in $\mathscr{P}_{d}$.

The strictly inadmissible monomial (see [21]). A monomial $u \in \mathscr{P}_{d}$ is said to be strictly inadmissible if and only if there exist monomials $x_{1}, x_{2}, \ldots, x_{k}$ such that $x_{j}<u$ for $1 \leq j \leq k$ and $u=\sum_{1 \leq j \leq k} x_{j}+\sum_{1 \leq t \leq 2^{s}-1} S q^{t}\left(y_{t}\right)$, where $s=\max \left\{i \in \mathbb{Z}: \omega_{i}(u)>0\right\}$ and suitable polynomials $y_{t} \in \mathscr{P}_{d}$.

Note that every the strictly inadmissible monomial is inadmissible but the converses not generally true (see a counter-example in [41]). The following result is used to study $Q \mathscr{P}_{5}$ in the next section.

Theorem 2.2.1 (see [21]). Let $x, y$ and $u$ be monomials in $\mathscr{P}_{d}$ such that $\omega_{i}(x)=0$ for $i>r>$ $0, \omega_{t}(u) \neq 0$ and $\omega_{i}(u)=0$ for $i>t>0$. Then, if $u$ is inadmissible, then $x u^{2^{r}} i s$, too. Furthermore, if $u$ is strictly inadmissible, then $u y^{2^{t}}$ is, too.

Let $\mathscr{P}_{d}^{0}$ and $\mathscr{P}_{d}^{+}$denote the $\mathcal{A}_{2}$-submodules of $\mathscr{P}_{d}$ spanned all the monomials $x_{1}^{t_{1}} x_{2}^{t_{2}} \ldots x_{d}^{t_{d}}$ such that $t_{1} t_{2} \ldots t_{d}=0$, and $t_{1} t_{2} \ldots t_{d}>0$, respectively. Denote by $Q \mathscr{P}_{d}^{0}:=\mathbb{Z} / 2 \otimes_{\mathcal{A}_{2}} \mathscr{P}_{d}^{0}$, and by $Q \mathscr{P}_{d}^{+}:=\mathbb{Z} / 2 \otimes_{\mathcal{A}_{2}} \mathscr{P}_{d}^{+}$. Then, we can see that $Q \mathscr{P}_{d}=Q \mathscr{P}_{d}^{0} \bigoplus Q \mathscr{P}_{d}^{+}$. We end this section by establishing a formula below on the dimension of $Q \mathscr{P}_{d}^{0}$ in degree $n$, which will be used in the next section. Note that this formula is similar to the one of [29].

Let $\mathcal{J}=\left(j_{1}, j_{2}, \ldots, j_{r}\right)$, where $1 \leq j_{1}<\ldots<j_{r} \leq d, 1 \leq r \leq d-1$, and let $r:=\ell(\mathcal{J})$ be the length of $\mathcal{J}$. We denote $\mathscr{P}_{\mathcal{J}}=\left\langle\left\{x_{j_{1}}^{t_{1}} x_{j_{2}}^{t_{2}} \ldots x_{j_{r}}^{t_{r}} \mid t_{s} \in \mathbb{N}, s=1,2, \ldots, r\right\}\right\rangle \subset \mathscr{P}_{d}$. Then, $\mathscr{P}_{\mathcal{J}}$ is $\mathcal{A}_{2}$-submodule of $\mathscr{P}_{d}$. Moreover, it is isomorphic to $\mathscr{P}_{r}$. Straightforward calculations indicate that

$$
Q \mathscr{P}_{d}^{0}=\bigoplus_{1 \leq r \leq d-1} \bigoplus_{\ell(\mathcal{J})=r} Q \mathscr{P}_{\mathcal{J}}^{+},
$$

where $\mathscr{P}_{\mathcal{J}}^{+}=\left\langle\left\{x_{j_{1}}^{t_{1}} x_{j_{2}}^{t_{2}} \ldots x_{j_{r}}^{t_{r}} \in \mathscr{P}_{\mathcal{J}} \mid t_{1} t_{2} \ldots t_{r}>0,1 \leq r \leq d-1\right\}\right\rangle$. It is easily seen that $\operatorname{dim}\left(Q \mathscr{P}_{\mathcal{J}}^{+}\right)_{n}=\operatorname{dim}\left(Q \mathscr{P}_{r}^{+}\right)_{n}$ for all $n$, and that $\left(\begin{array}{l}d \\ r\end{array}\right)$ is the number of the sequences $\mathcal{J}$ of length $r$. Therefore, we get

$$
\operatorname{dim}\left(Q \mathscr{P}_{d}^{0}\right)_{n}=\sum_{1 \leq r \leq d-1}\left(\begin{array}{l}
d \\
r
\end{array}\right) \operatorname{dim}\left(Q \mathscr{P}_{r}^{+}\right)_{n}
$$




\section{Generators of the $\mathcal{A}_{2}$-module $\mathscr{P}_{5}$ in the generic degree $5\left(2^{t}-1\right)+8.2^{t}$}

In this section we study the structure of $Q \mathscr{P}_{5}$ in degree $5\left(2^{t}-1\right)+8.2^{t}$ for $t$ a positive integer. More explicitly, we will prove Theorem 1.1 as given at the beginning. We first review some homomorphisms and related results, Sum's conjecture [65] and Singer's criterion on $\mathcal{A}_{2}$-decomposable [57].

\subsection{Singer's criterion on $\mathcal{A}_{2}$-decomposable}

Definition 3.1.1. A monomial $z=x_{1}^{t_{1}} x_{2}^{t_{2}} \ldots x_{d}^{t_{d}}$ in $\mathscr{P}_{d}$ is called a spike if $t_{j}=2^{a_{j}}-1$ for $a_{j}$ a non-negative integer and $1 \leq j \leq d$. If $z$ is a spike with $a_{1}>a_{2}>\ldots>a_{r-1} \geq a_{r}>0$ and $a_{j}=0$ for $j>r$, then it is called a minimal spike.

Proposition 3.1.2 (see $[39,41]$ ). All the spikes in $\mathscr{P}_{d}$ are admissible and their weight vectors are weakly decreasing. Furthermore, if a weight vector $\omega=\left(\omega_{1}, \omega_{2}, \ldots\right)$ is weakly decreasing and $\omega_{1} \leq d$, then there is a spike $z$ in $\mathscr{P}_{d}$ such that $\omega(z)=\omega$.

We refer the reader to [41] for the detailed proofs of the proposition. Singer showed in [58] that if $\mu(n) \leq d$, then there exists uniquely a minimal spike of degree $n$ in $\mathscr{P}_{d}$. Further, we have the following, which is one of the important keys for examining the hit monomials in generic degrees.

Theorem 3.1.3 (Singer [58]). Suppose that $X \in \mathscr{P}_{d}$ is a monomial of degree $n$, where $\mu(n) \leq d$. Let $z$ be the minimal spike of degree $n$ in $\mathscr{P}_{d}$. If $\omega(X)<\omega(z)$, then $X$ is $\mathcal{A}_{2}$-decomposable.

\subsection{Some homomorphisms and Sum's conjecture}

For $1 \leq k \leq d$, we define the map $\rho_{(k, d)}: \mathscr{P}_{d-1} \rightarrow \mathscr{P}_{d}$ of $\mathbb{Z} / 2$-algebras by setting

$$
\rho_{(k, d)}\left(x_{j}\right)= \begin{cases}x_{j} & \text { if } 1 \leq j<k \\ x_{j+1} & \text { if } k \leq j<d\end{cases}
$$

We consider the following set

$$
\mathcal{N}_{d}:=\left\{(k ; \mathscr{K}) \mid \mathscr{K}=\left(k_{1}, k_{2}, \ldots, k_{r}\right), 1 \leqslant k<k_{1}<k_{2}<\ldots<k_{r} \leq d, 0 \leq r<d\right\},
$$

where by convention, $\mathscr{K}=\emptyset$, if $r=0$. Denote by $r=\ell(\mathscr{K})$ the length of $\mathscr{K}$. For any $(k ; \mathscr{K}) \in \mathcal{N}_{d}$, we have the projections (see [62]) $\pi_{(k ; \mathscr{K})}: \mathscr{P}_{d} \rightarrow \mathscr{P}_{d-1}$, which are determined by

$$
\pi_{(k ; \mathscr{K})}\left(x_{j}\right)= \begin{cases}x_{j} & \text { if } 1 \leq j<k, \\ \sum_{p \in \mathscr{K}} x_{p-1} & \text { if } j=k, \\ x_{j-1} & \text { if } k<j \leq d .\end{cases}
$$

Note that $\rho_{(k, d)}$ and $\pi_{(k ; \mathscr{K})}$ are also the homomorphisms of the $\mathcal{A}_{2}$-modules. In particular, we have $\pi_{(k ; \emptyset)}\left(x_{k}\right)=0$ for $1 \leq k \leq d$ and $\pi_{(k ; \mathscr{K})}\left(\rho_{(k, d)}(u)\right)=u$ for any $u \in \mathscr{P}_{d-1}$.

Proposition 3.2.1 (see [39]). If $x$ is a monomial in $\mathscr{P}_{d}$, then $\pi_{(k ; \mathscr{K})}(x) \in \mathscr{P}_{d-1}(\omega(x))$.

This result implies that if $\omega$ is a weight vector and $x \in \mathscr{P}_{d}(\omega(x))$, then $\pi_{(k ; \mathscr{K})}(x) \in \mathscr{P}_{d-1}(\omega)$. Furthermore, $\pi_{(k ; \mathscr{K})}$ passes to a homomorphism from $Q \mathscr{P}_{d}(\omega)$ to $Q \mathscr{P}_{d-1}(\omega)$.

Let $(k ; \mathscr{K}) \in \mathcal{N}_{d}, 0<r<d$, and let $x_{(\mathscr{K}, u)}=x_{k_{u}}^{2^{r-1}+2^{r-2}+\cdots+2^{r-u}} \prod_{u<m<r} x_{k_{m}}^{2^{r-m}}$ for $1 \leq u \leq$ $r, x_{(\emptyset, 1)}=1$. In [62], Sum defined an $\mathbb{Z} / 2$-linear transformation $\phi_{(k ; \mathscr{K})}: \mathscr{P}_{d-1} \rightarrow \mathscr{P}_{d}$, which is determined by

$$
\phi_{(k ; \mathscr{K})}(x)= \begin{cases}\rho_{(k, d)}(x) & \text { if } \mathscr{K}=\emptyset, \\ \frac{x_{k}^{2^{r}-1} \rho_{(k, d)}(x)}{x_{(\mathscr{K}, u)}} & \text { if there exist } u \text { such that: } \\ & t_{k_{1}-1}=t_{k_{2}-1}=\ldots=t_{k_{(u-1)}-1}=2^{r}-1, \\ & t_{k_{u}-1}>2^{r}-1, \\ & \alpha_{r-m}\left(t_{k_{u}-1}\right)=1, \forall m, 1 \leq m \leq u, \text { and } \\ & \alpha_{r-m}\left(t_{k_{m}-1}\right)=1, \forall m, u<m \leq r, \\ & \text { otherwise, }\end{cases}
$$

for any $x=x_{1}^{t_{1}} x_{2}^{t_{2}} \ldots x_{d-1}^{t_{d-1}}$ in $\mathscr{P}_{d-1}$. Note that $\phi_{(k ; \mathscr{K})}$ is not an $\mathcal{A}_{2}$-homomorphism in general. Moreover, for each $x \in \mathscr{P}_{d-1}$, if $\phi_{(k ; \mathscr{K})}(x) \neq 0$, then $\omega\left(\phi_{(k ; \mathscr{K})}(x)\right)=\omega(x)$.

From now on, we adopt the following notations: For a natural number $d$, we consider $\Gamma_{d}=$ $\{1,2, \ldots, d\}, X_{(S, d)}=X_{\left(\left\{s_{1}, s_{2}, \ldots, s_{r}\right\}, d\right)}=\prod_{s \in \Gamma_{d} \backslash S} x_{s}$, where $S=\left\{s_{1}, s_{2}, \ldots, s_{r}\right\} \subseteq \Gamma_{d}$. In particular, $X_{\left(\Gamma_{d}, d\right)}=1, \quad X_{(\emptyset, d)}=x_{1} x_{2} \ldots x_{d}, X_{(\{s\}, d)}=x_{1} \ldots \hat{x}_{s} \ldots x_{d}, 1 \leq s \leq d$. Now consider $X=x_{1}^{t_{1}} x_{2}^{t_{2}} \ldots x_{d}^{t_{d}} \in \mathscr{P}_{d}$ and let $S_{j}(X)=\left\{s \in \Gamma_{d}: \alpha_{j}\left(t_{s}\right)=0\right\}$ for $j \geq 0$. Then, by a simple computation, we get $X=\prod_{j \geq 0} X_{\left(S_{j}(X), d\right)}^{2^{j}}$.

The following examples on the map $\phi_{(k ; \mathscr{K})}$ can be found in [62]. However, we present them in more detail. 
(i) Let $\mathscr{K}=(j)$ and $1 \leq k<j \leq d$. Then, for any the monomial $x=x_{1}^{a_{1}} x_{2}^{a_{2}} \ldots x_{d-1}^{a_{d-1}} \in \mathscr{P}_{d-1}$ and $\alpha_{0}\left(a_{j-1}\right)=1$, we conclude $\phi_{(k ; \mathscr{K})}(x)=\frac{x_{k} \rho_{(k, d)}(x)}{x_{j}}$.

(ii) Let $m$ be a positive integer and let $x=Y^{2^{m}-1} y^{2^{m}}$ with $y=x_{1}^{b_{1}} x_{2}^{b_{2}} \ldots x_{d-1}^{b_{d-1}}$ and $Y=$ $X_{(\{d\}, d)}=x_{1} x_{2} \ldots x_{d-1} \in \mathscr{P}_{d-1}$. Then if $m>r=\ell(\mathscr{K})$ and $u=1$ then

$$
\phi_{(k ; \mathscr{K})}(x)=\phi_{(k ; \mathscr{K})}\left(Y^{2^{m}-1}\right)\left(\rho_{(k, d)}(y)\right)^{2^{m}}=x_{k}^{2^{r}-1} \prod_{1 \leqslant t \leqslant r} x_{k_{t}}^{2^{m}-2^{r-t}-1} X_{\left(\left\{k, k_{1}, \ldots, k_{r}\right\}, d\right)}^{2^{m}-1}\left(\rho_{(k, d)}(y)\right)^{2^{m}} .
$$

Indeed, since $\rho_{(k, d)}$ is an $\mathbb{Z} / 2$-algebras homomorphism,

$$
\rho_{(k, d)}(x)=\rho_{(k, d)}\left(X^{2^{m}-1}\right)\left(\rho_{(k, d)}(y)\right)^{2^{m}}, 1 \leq k \leq d .
$$

Since $Y^{2^{m}-1}=x_{1}^{2^{m}-1} \ldots x_{d-1}^{2^{m}-1}$ and $2^{d}-1>2^{r}-1$, for each $(k ; \mathscr{K}), \mathscr{K}=\left(k_{1}, k_{2}, \ldots, k_{r}\right)$ and $u=1$, we have

$$
\begin{aligned}
\phi_{(k ; \mathscr{K})}\left(Y^{2^{m}-1}\right) & =\frac{x_{k}^{2^{r}-1} x_{2}^{2^{m}-1} \ldots x_{k_{1}}^{2^{m}-1} \ldots x_{k_{r}}^{2^{m}-1} \ldots x_{d}^{2^{m}-1}}{x_{k_{1}}^{2^{r-1}} x_{k_{2}}^{2^{r-2}} \ldots x_{k_{r}}^{2^{r-r}}} \\
& =x_{k}^{2^{r}-1} x_{k_{1}}^{2^{m}-2^{r^{-1}}-1} \ldots x_{k_{2}}^{2^{m}-2^{r-2}-1} \ldots x_{k_{r}}^{2^{m}-2^{r-r}-1} X_{\left(\left\{k, k_{1}, \ldots, k_{r}\right\}, d\right)}^{2^{m}-1} \\
& =x_{k}^{2^{r}-1} \prod_{1 \leqslant t \leqslant r} x_{k_{t}}^{2^{m}-2^{r-t}-1} X_{\left(\left\{k, k_{1}, \ldots, k_{r}\right\}, d\right)}^{2^{m}-1} .
\end{aligned}
$$

Then, one gets

$$
\begin{aligned}
\phi_{(k ; \mathscr{K})}(x) & =\phi_{(k ; \mathscr{K})}\left(Y^{2^{m}-1} y^{2^{m}}\right) \\
& =\frac{x_{k}^{2^{r}-1} \rho_{(k, d)}\left(X^{2^{m}-1} y^{2^{m}}\right)}{x_{(\mathscr{K} ; 1)}}=\left(\frac{x_{k}^{2^{r}-1} \rho_{(k, d)}\left(Y^{2^{m}-1}\right)}{x_{(\mathscr{K} ; 1)}}\right)\left(\rho_{(k, d)}(y)\right)^{2^{m}} \\
& =\phi_{(k ; \mathscr{K})}\left(X^{2^{m}-1}\right)\left(\rho_{(k, d)}(y)\right)^{2^{m}} \\
& =x_{k}^{2^{r}-1} \prod_{1 \leqslant t \leqslant r} x_{k_{t}}^{2^{m}-2^{r-t}-1} X_{\left(\left\{k, k_{1}, \ldots, k_{r}\right\}, d\right)}^{2^{m}-1}\left(\rho_{(k, d)}(y)\right)^{2^{m}} .
\end{aligned}
$$

Now, if $m=r, b_{j-1}=0, j=k_{1}, k_{2}, \ldots, k_{u-1}$ and $b_{k_{u-1}}>0$ then for each $(k ; \mathscr{K})$ and $1 \leq u \leq$ $r=m$, we deduce

$$
\phi_{(k ; \mathscr{K})}(x)=\phi_{\left(k_{u} ;\left\{k_{u+1}, \ldots, k_{m}\right\}\right)}\left(Y^{2^{m}-1}\right)\left(\rho_{(k, d)}(y)\right)^{2^{m}} .
$$

Indeed, we have

$$
\begin{aligned}
Y^{2^{m}-1} y^{2^{m}} & =\left(x_{1} \ldots x_{d-1}\right)^{2^{m}-1}\left(x_{1}^{b_{1}} \ldots \hat{x}_{k_{1}}^{b_{k_{1}}} \ldots \hat{x}_{k_{(u-1)}-1}^{b_{k_{(u-1}-1}} x_{k_{u}-1}^{b_{k_{u}-1}} \ldots x_{d-1}^{b_{d-1}}\right)^{2^{m}} \\
& =\left(x_{1} \ldots x_{k_{1}} \ldots x_{k_{u-1}-1} x_{k_{u}-1} \ldots x_{d-1}\right)^{2^{m}-1}\left(x_{1}^{b_{1}} \ldots \hat{x}_{k_{1}}^{b_{k_{1}}} \ldots \hat{x}_{k_{(u-1)}-1}^{b_{k_{(u-1}-1}} x_{k_{u}-1}^{b_{k_{u}-1}} \ldots x_{d-1}^{b_{d-1}}\right)^{2^{m}} .
\end{aligned}
$$

Then, we get

$$
\begin{aligned}
\phi_{(k ; \mathscr{K})}(x)= & \frac{x_{k}^{2^{m}-1} \rho_{(k, d)}\left(Y^{2^{m}-1}\right)}{x_{k_{u}}^{2^{m-1}+2^{m-2}+\cdots+2^{m-u}} \prod_{u<t \leqslant r} x_{k_{t}}^{2^{m-t}}}\left(\rho_{(k, d)}(y)\right)^{2^{m}} . \\
= & \frac{x_{k}^{2^{m}-1} x_{2}^{2^{m}-1} \ldots x_{k_{u-1}}^{2^{m}-1} x_{k_{u}}^{2^{m}}-1 \prod_{u+1 \leqslant t \leqslant m} x_{k_{t}}^{2^{m}-1} \ldots x_{d}^{2^{m}-1}}{x_{k_{u}}^{2^{m-1}+2^{m-2}+\cdots+2^{m-u}} \prod_{u<t \leqslant m} x_{k_{t}}^{2^{m-t}}}\left(\rho_{(k, d)}(y)\right)^{2^{m}} \\
= & x_{k}^{2^{m}-1} x_{2}^{2^{m}-1} \ldots x_{k_{u-1}}^{2^{m}-1} x_{k_{u}}^{2^{m-(u+1)}+\cdots+2^{m-m}} \ldots x_{d}^{2^{m}-1} \\
& \prod_{u+1 \leqslant t \leqslant m} x_{k_{t}}^{2^{m}-2^{m-t}-1} \ldots x_{d}^{2^{m}-1}\left(\rho_{(k, d)}(y)\right)^{2^{m}} \\
= & \left(x_{k_{u}}^{2^{m-(u+1)}+\cdots+2^{m-m}} \prod_{\left(k_{u} ;\left\{k_{u+1}, \ldots, k_{m}\right\}\right)}\left(Y^{2^{m}}-1\right)\left(\rho_{(k, d)}(y)\right)^{2^{m}} .\right.
\end{aligned}
$$

We end this subsection by reviewing Sum's conjecture [65] on the relation between the admissible monomials for the polynomial algebras.

For a subset $\mathcal{U} \subset \mathscr{P}_{d-1}$, we denote

$$
\begin{aligned}
& \bar{\Phi}^{0}(\mathcal{U})=\bigcup_{1 \leq k \leq d} \phi_{(k ; \emptyset)}(\mathcal{U})=\bigcup_{1 \leq k \leq d} \rho_{(k, d)}(\mathcal{U}), \\
& \bar{\Phi}^{+}(\mathcal{U})=\bigcup_{(k ; \mathscr{K}) \in \mathcal{N}_{d}, 0<\ell(\mathscr{K})<d} \phi_{(k ; \mathscr{K})}(\mathcal{U}) \backslash \mathscr{P}_{d}^{0}, \\
& \bar{\Phi}(\mathcal{U})=\bar{\Phi}^{0}(\mathcal{U}) \bigcup \bar{\Phi}^{+}(\mathcal{U}) .
\end{aligned}
$$

Since $\rho_{(k, d)}$ is a homomorphism of the $\mathcal{A}_{2}$-modules, if $\mathcal{U}$ is a minimal set of generators for the $\mathcal{A}_{2}$-module $\mathscr{P}_{d-1}$ in degree $n$, then $\bar{\Phi}^{0}(\mathcal{U})$ is also a minimal set of generators for the $\mathcal{A}_{2}$-module $\mathscr{P}_{d}^{0}$ in degree $n$. 
Now, for a polynomial $f \in \mathscr{P}_{d}$, we denote by $[f]$ the classes in $Q \mathscr{P}_{d}$ represented by $f$. If $\omega$ is a weight vector and $f \in \mathscr{P}_{d}(\omega)$, then denote by $[f]_{\omega}$ the classes in $Q \mathscr{P}_{d}(\omega)$ represented by $f$. For a subset $\mathscr{B} \subset \mathscr{P}_{d}$, we denote $[\mathscr{B}]=\{[f]: f \in \mathscr{B}\}$. If $\mathscr{B} \subset \mathscr{P}_{d}(\omega)$, then we set $[\mathscr{B}]_{\omega}=\left\{[f]_{\omega}: f \in \mathscr{B}\right\}$.

Denote by $\mathscr{B}_{d}(n)$ the set of all admissible monomials of degree $n$ in $\mathscr{P}_{d}$. Thus when we write $x \in \mathscr{B}_{d}(n)$ we mean that it is an admissible monomial of degree $n$. We set

$$
\mathscr{B}_{d}^{0}(n):=\mathscr{B}_{d}(n) \cap\left(\mathscr{P}_{d}^{0}\right)_{n}, \mathscr{B}_{d}^{+}(n):=\mathscr{B}_{d}(n) \cap\left(\mathscr{P}_{d}^{+}\right)_{n} .
$$

If $\omega$ is a weight vector of degree $n$, we set

$$
\mathscr{B}_{d}(\omega):=\mathscr{B}_{d}(n) \cap \mathscr{P}_{d}(\omega), \mathscr{B}_{d}^{0}(\omega):=\mathscr{B}_{d}(\omega) \cap\left(\mathscr{P}_{d}^{0}\right)_{n}, \mathscr{B}_{d}^{+}(\omega):=\mathscr{B}_{d}(\omega) \cap\left(\mathscr{P}_{d}^{+}\right)_{n} .
$$

Then, $\left[\mathscr{B}_{d}(\omega)\right]_{\omega},\left[\mathscr{B}_{d}^{0}(\omega)\right]_{\omega}$ and $\left[\mathscr{B}_{d}^{+}(\omega)\right]_{\omega}$ are respectively the bases of the $\mathbb{Z} / 2$-vector spaces

$$
Q \mathscr{P}_{d}(\omega), Q \mathscr{P}_{d}^{0}(\omega):=Q \mathscr{P}_{d}(\omega) \cap\left(Q \mathscr{P}_{d}^{0}\right)_{n} \text { and } Q \mathscr{P}_{d}^{+}(\omega):=Q \mathscr{P}_{d}(\omega) \cap\left(Q \mathscr{P}_{d}^{+}\right)_{n} .
$$

Throughout this paper, to prove a certain subset of $Q \mathscr{P}_{d}$ is linearly independent, we use a result in Sum [62] combining with Theorem 3.1.3 (Singer's criterion on the $\mathcal{A}_{2}$-decomposable) and Proposition 3.2.1. More precisely, let $\mathscr{B}$ be a finite subset of $\mathscr{P}_{d}$ consisting of some monomials of degree $n$. Denote by $|\mathscr{B}|$ the cardinal of $\mathscr{B}$. To prove the set $[\mathscr{B}]$ is linearly independent in $\left(Q \mathscr{P}_{d}\right)_{n}$, we denote the elements of $\mathscr{B}$ by $y_{n, i}, 1 \leq i \leq m=|\mathscr{B}|$ and assume that there is a linear relation

$$
\mathcal{S}=\sum_{1 \leq i \leq m} \gamma_{i} y_{n, i}=0 \text { modulo }\left(\mathcal{A}_{2}^{+} \mathscr{P}_{d}+\mathscr{P}_{d}^{-}(\omega)\right),
$$

with $\gamma_{i} \in \mathbb{Z} / 2$ for all $i, 1 \leq i \leq m$. For $(k ; \mathscr{K}) \in \mathcal{N}_{d}$, we explicitly compute $\pi_{(k ; \mathscr{K})}(\mathcal{S})$ in terms of the admissible monomials in $\mathscr{P}_{d-1}\left(\operatorname{modulo}\left(\mathcal{A}_{2}^{+} \mathscr{P}_{d-1}+\mathscr{P}_{d-1}^{-}(\omega)\right)\right)$. Computing from some relations $\pi_{(k ; \mathscr{K})}(\mathcal{S})=0$ modulo $\left(\mathcal{A}_{2}^{+} \mathscr{P}_{d-1}+\mathscr{P}_{d-1}^{-}(\omega)\right)$ with $(k ; \mathscr{K}) \in \mathcal{N}_{d}$, we obtain $\gamma_{i}=0$ for all $i$.

In [65], Sum made the following conjecture, which plays an important role in studying the minimal set of $\mathcal{A}_{2}$-module $\mathscr{P}_{d}$ in certain generic degree.

Conjecture 3.2.2 (Sum [65]). If $\omega$ is a weight vector, then $\bar{\Phi}\left(\mathscr{B}_{d-1}(\omega)\right) \subseteq \mathscr{B}_{d}(\omega)$.

Obviously, if this conjecture is true, then $\bar{\Phi}\left(\mathscr{B}_{d-1}(n)\right) \subseteq \mathscr{B}_{d}(n)$ for any positive integer $n$. In other words, if $x \in \mathscr{B}_{d-1}(n)$, then $\phi_{(k ; \mathscr{K})}(x) \in \mathscr{B}_{d}(n)$. By previous results of Peterson [37], Kameko [21] and Sum [62], the conjecture is true for $d \leq 4$. In particular, we have the remark below.

Remark 3.2.3. Consider the spike monomial $Y=X_{(\{d\}, d)}=x_{1} x_{2} \ldots x_{d-1} \in \mathscr{P}_{d-1}$. Let $m$ be a positive integer such that $m>r=\ell(\mathscr{K})$. Then, from the above calculations, we have

$$
\phi_{(k ; \mathscr{K})}\left(Y^{2^{m}-1}\right)=x_{k}^{2^{r}-1} \prod_{1 \leq t \leq r} x_{i_{t}}^{2^{m}-2^{r-t}-1} X_{\left(\left\{k, k_{1}, \ldots, k_{r}\right\}, d\right)}^{2^{m}-1} .
$$

It is easy to see that $\omega(Y)=\underbrace{(d-1, d-1, \ldots, d-1)}_{m \text { times of }(d-1)}$. Based on the results in $[40,66]$, the set $\left\{\phi_{(k ; \mathscr{K})}\left(Y^{2^{m}-1}\right):(k ; \mathscr{K}) \in \mathcal{N}_{d}\right\}$ is a basis of $Q \mathscr{P}_{d}(\omega(Y))$. Note that this also holds true for $m \leq r$ (see Sum [66]). By Proposition 3.1.2, $Y^{2^{m}-1}$ is admissible. These data imply that Sum's conjecture is true for the weight vector $\omega(Y)$, where $d$ is an arbitrary positive integer.

In [65], Sum showed that Conjecture 3.2.2 is true for $d=5$ and any weight vector of generic degree of (1.1) with $r=d=5, s=10$ and $t \geq 0$. In the next subsection, we will show that this conjecture is also satisfying for $d=5$ and in generic degree of Theorem 1.1.

\subsection{Proof of Theorem 1.1}

As shown in Sect.1, we have $\mu\left(13.2^{t}-5\right)=5$ for every $t>2$, hence the inverse function $\widetilde{\phi}:\left(Q \mathscr{P}_{5}\right)_{13.2^{t-1}-5} \rightarrow\left(Q \mathscr{P}_{5}\right)_{13.2^{t}-5}$ of $\left(\widetilde{S q_{*}^{0}}\right)_{\left(5,13.2^{t}-5\right)}$ defined by $\widetilde{\phi}([u])=\left[X_{(\emptyset, 5)} u^{2}\right]$ for all $[u] \in$ $\left(Q \mathscr{P}_{5}\right)_{13.2^{t-1}-5}, t>2$. On the other hand, since the iterated Kameko squaring operation

$$
\left(\widetilde{S q_{*}^{0}}\right)_{\left(5,13.2^{t}-5\right)}^{t-2}:\left(Q \mathscr{P}_{5}\right)_{13.2^{t}-5} \rightarrow\left(Q \mathscr{P}_{5}\right)_{13.2^{2}-5}
$$

is an $\mathbb{Z} / 2$-vector space isomorphism for every $t \geq 2$, a basis of $Q \mathscr{P}_{5}$ in degree $13.2^{t}-5$ is the set

$$
\left[\mathscr{B}_{5}\left(13.2^{t}-5\right)\right]=\widetilde{\phi}^{t-2}\left(\left[\mathscr{B}_{5}\left(13.2^{2}-5\right)\right]\right)
$$

for $t>2$. Thus, we need only to find the minimal set of $\mathbb{Z} / 2$-generators for $\left(Q \mathscr{P}_{5}\right)_{13.2^{t}-5}$ with $t \in\{0,1,2\}$. It has been determined by Tín [69] for $t=0$. Note that our methods of studying $Q \mathscr{P}_{5}$ in this paper are different from the ones of Tín. 
3.3.1. The case $t=1$. Consider Kameko's homomorphism $\left(\widetilde{S q_{*}^{0}}\right)_{(5,21)}:\left(Q \mathscr{P}_{5}\right)_{21} \longrightarrow\left(Q \mathscr{P}_{5}\right)_{8}$. We know that it is an epimorphism of $\mathbb{Z} / 2$-vector spaces, hence $\left(Q \mathscr{P}_{5}\right)_{21} \cong \operatorname{Ker}\left(\widetilde{S q_{*}^{0}}\right)_{(5,21)} \bigoplus\left(Q \mathscr{P}_{5}\right)_{8}$. We note that $\operatorname{Ker}\left(\widetilde{S q_{*}^{0}}\right)_{(5,21)}$ is isomorphic to $\left(Q \mathscr{P}_{5}^{0}\right)_{21} \bigoplus\left(\operatorname{Ker}\left(\widetilde{S q_{*}^{0}}\right)_{(5,21)} \cap\left(Q \mathscr{P}_{5}^{+}\right)_{21}\right)$. From the calculations of $Q \mathscr{P}_{d}$ in degree 21 for $1 \leq d \leq 4$ (see [21], [37], [62]) and $Q \mathscr{P}_{5}$ in degree 8 (see [69]), we have

$$
\left|\mathscr{B}_{1}^{+}(21)\right|=0,\left|\mathscr{B}_{2}^{+}(21)\right|=0,\left|\mathscr{B}_{3}^{+}(21)\right|=7,\left|\mathscr{B}_{4}^{+}(21)\right|=66,\left|\mathscr{B}_{5}(8)\right|=174 .
$$

Note that $\left(Q \mathscr{P}_{3}\right)_{21} \cong\left(Q \mathscr{P}_{3}\right)_{3}$ and $\mathscr{B}_{3}(21)=\mathscr{B}_{3}^{+}(21)=\widetilde{\varphi}^{2}\left(\mathscr{B}_{3}(3)\right)$ with the $\mathbb{Z} / 2$-linear map $\widetilde{\varphi}: \mathscr{P}_{3} \rightarrow \mathscr{P}_{3}$, determined by $\widetilde{\varphi}(u)=X_{(\emptyset, 3)} u^{2}, \forall u \in \mathscr{P}_{3}$. As well known,

$$
\left(Q \mathscr{P}_{5}^{0}\right)_{21}=\bigoplus_{1 \leq r \leq 4} \bigoplus_{1 \leq j \leq\left(\begin{array}{c}
5 \\
r
\end{array}\right)}\left(Q \mathscr{P}_{r}^{+}\right)_{21},
$$

hence $\operatorname{dim}\left(Q \mathscr{P}_{5}^{0}\right)_{21}=\left(\begin{array}{l}5 \\ 3\end{array}\right) .7+\left(\begin{array}{l}5 \\ 4\end{array}\right) \cdot 66=400$. Moreover, a direct computation shows that

$$
\mathscr{B}_{5}^{0}(21)=\bar{\Phi}^{0}\left(\mathscr{B}_{4}(21)\right)=\left\{y_{21, i}: 1 \leq i \leq 400\right\},
$$

where the monomials $y_{21, i}, 1 \leq i \leq 400$, are listed in Sect.6.2 of the online version [44].

Proposition 3.3.1. The set $\left\{\left[y_{21, i}\right]: 401 \leq i \leq 666\right\}$ is the basis of the $\mathbb{Z} / 2$-vector space $\operatorname{Ker}\left(\widetilde{S q_{*}^{0}}\right)_{(5,21)} \cap\left(Q \mathscr{P}_{5}^{+}\right)_{21}$. Here the monomials $y_{i}:=y_{21, i}, 401 \leq i \leq 666$, which are determined in Sect. 6.3 of [44].

Combining Proposition 3.3.1 and the above data, we deduce that the $\mathbb{Z} / 2$-vector space $\left(Q \mathscr{P}_{5}\right)_{21}$ is 840-dimensional. This completes the proof of the theorem for the case $t=1$.

We now need to some results for the proof of Proposition 3.3.1. First, we have the following lemma.

Lemma 3.3.2. If $u \in \mathscr{B}_{5}(21)$ and $[u] \in \operatorname{Ker}\left(\widetilde{S q_{*}^{0}}\right)_{(5,21)}$, then the weight vector of $u$ is either $\omega(u)=(3,3,1,1)$ or $\omega(u)=(3,3,3)$.

Proof. Note that $x_{1}^{15} x_{2}^{3} x_{3}^{3} \in\left(\mathscr{P}_{5}\right)_{21}$ is the minimal spike, and that by Proposition 3.1.2, it is an admissible monomial. Moreover, $\omega\left(x_{1}^{15} x_{2}^{3} x_{3}^{3}\right)=(3,3,1,1)$. Since $[u] \neq[0]$, by Theorem 3.1 .3 , we get either $\omega_{1}(u)=3$ or $\omega_{1}(u)=5$. If $\omega_{1}(u)=5$ then $u=X_{(\emptyset, 5)} y^{2}$ with $y$ a monomial of degree 8 in $\mathscr{P}_{5}$. Since $u$ is admissible, by Theorem 2.2.1, one gets $y \in \mathscr{B}_{5}(8)$. So $\left(\widetilde{S q_{*}^{0}}\right)_{(5,21)}([u])=[y] \neq[0]$. This contradicts the fact that $[u] \in \operatorname{Ker}\left(\widetilde{S q_{*}^{0}}\right)_{(5,21)}$. Hence, $\omega_{1}(u)=3$. Then, we have $u=X_{(\{i, j\}, 5)} y_{1}^{2}$ with $1 \leq i<j \leq 5$ and $y_{1} \in \mathscr{B}_{5}(9)$. Since $y_{1}$ is admissible, according to a result in [?], we have either $\omega\left(y_{1}\right)=(3,1,1)$ or $\omega\left(y_{1}\right)=(3,3)$. The lemma is proved.

As an immediate consequence, we see that the dimension of $\left(\operatorname{Ker}\left(\widetilde{S q_{*}^{0}}\right)_{(5,21)} \cap\left(Q \mathscr{P}_{5}^{+}\right)_{21}\right)$ is equal to the sum of dimensions of $Q \mathscr{P}_{5}^{+}(3,3,1,1)$ and $Q \mathscr{P}_{5}^{+}(3,3,3)$. This leads us to determine the subspaces $Q \mathscr{P}_{5}^{+}(\omega)$, where the weight vectors $\omega$ are $(3,3,1,1)$ and $(3,3,3)$.

The following lemma is an immediate corollary from a result in [62].

Lemma 3.3.3. The following monomials are strictly inadmissible:

(i) $x_{i}^{2} x_{j} x_{k}^{3} x_{\ell}^{7}, x_{i}^{6} x_{j} x_{k}^{3} x_{\ell}^{3}, x_{i}^{2} x_{j}^{5} x_{k}^{3} x_{\ell}^{3}, x_{i}^{2} x_{j} x_{k}^{3} x_{\ell}^{3}$, where $1 \leq i<j \leq 5,1 \leq k, \ell \leq 5$, $k \neq \ell$, and $k, \ell \neq i, j$

(ii) $x_{i}^{3} x_{j}^{4} x_{k}^{3} x_{\ell}^{3}, 1 \leq i<j<k<\ell \leq 5$;

(iii) $\rho_{(k, 5)}(X), 1 \leq k \leq 5$, where $X$ is one of the following monomials:

$$
\begin{array}{llll}
x_{1}^{3} x_{2}^{4} x_{3}^{7} x_{4}^{7}, & x_{1}^{3} x_{2}^{7} x_{3}^{4} x_{4}^{7}, & x_{1}^{3} x_{2}^{7} x_{3}^{7} x_{4}^{4}, & x_{1}^{7} x_{2}^{3} x_{3}^{4} x_{4}^{7}, \\
x_{1}^{7} x_{2}^{3} x_{3}^{7} x_{4}^{4}, & x_{1}^{7} x_{2}^{7} x_{3}^{3} x_{4}^{4}, & x_{1}^{7} x_{2}^{8} x_{3}^{3} x_{4}^{3} .
\end{array}
$$

Lemma 3.3.4. The following monomials are strictly inadmissible:

(i) $x_{i}^{2} x_{j}^{2} x_{k} x_{\ell} x_{m}^{7}, x_{i}^{2} x_{j}^{6} x_{k} x_{\ell} x_{m}^{3}, x_{i}^{6} x_{j}^{2} x_{k} x_{\ell} x_{m}^{3}, x_{i}^{2} x_{j}^{2} x_{k}^{5} x_{\ell} x_{m}^{3}, x_{i}^{2} x_{j}^{4} x_{k} x_{\ell}^{3} x_{m}^{3}$, $x_{i}^{4} x_{j}^{2} x_{k} x_{\ell}^{3} x_{m}^{3}, x_{i}^{2} x_{j} x_{k}^{4} x_{\ell}^{3} x_{m}^{3}$, where $(i, j, k, \ell, m)$ is a permutation of $(1,2,3,4,5)$;

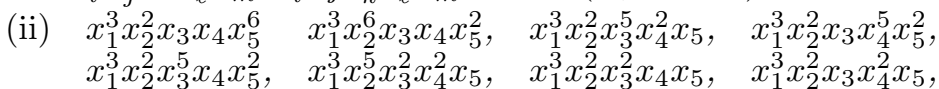
$x_{1}^{3} x_{2}^{2} x_{3} x_{4} x_{5}^{2}$.

Proof. Consider the monomials $X=x_{i}^{2} x_{j}^{2} x_{k} x_{\ell} x_{m}^{7}$ and $Y=x_{1}^{3} x_{2}^{2} x_{3} x_{4} x_{5}^{6}$. We prove that these monomials are strictly inadmissible. The others can be proved by a similar computation. Obviously, $\omega(X)=\omega(Y)=(3,3,1)$. By a direct computation using the Cartan formula, we obtain

$$
\begin{aligned}
X= & S q^{2}\left(x_{i} x_{j} x_{k} x_{\ell} x_{m}^{7}\right)+S q^{4}\left(x_{i} x_{j} x_{k} x_{\ell} x_{m}^{5}\right)+x_{i} x_{j} x_{k}^{2} x_{\ell}^{2} x_{m}^{7} \\
& +x_{i} x_{j}^{2} x_{k} x_{\ell}^{2} x_{m}^{7}+x_{i} x_{j}^{2} x_{k}^{2} x_{\ell} x_{m}^{7} \text { modulo }\left(\mathscr{P}_{5}^{-}(3,3,1)\right) ; \\
Y= & S q^{1}\left(x_{1}^{3} x_{2} x_{3} x_{4} x_{5}^{6}\right)+x_{1}^{3} x_{2} x_{3}^{2} x_{4} x_{5}^{6}+x_{1}^{3} x_{2} x_{3} x_{4}^{2} x_{5}^{6} \text { modulo }\left(\mathscr{P}_{5}^{-}(3,3,1)\right) .
\end{aligned}
$$

These equalities show that $X$ and $Y$ are strictly inadmissible. The lemma follows. 
Lemma 3.3.5. The following monomials are strictly inadmissible:

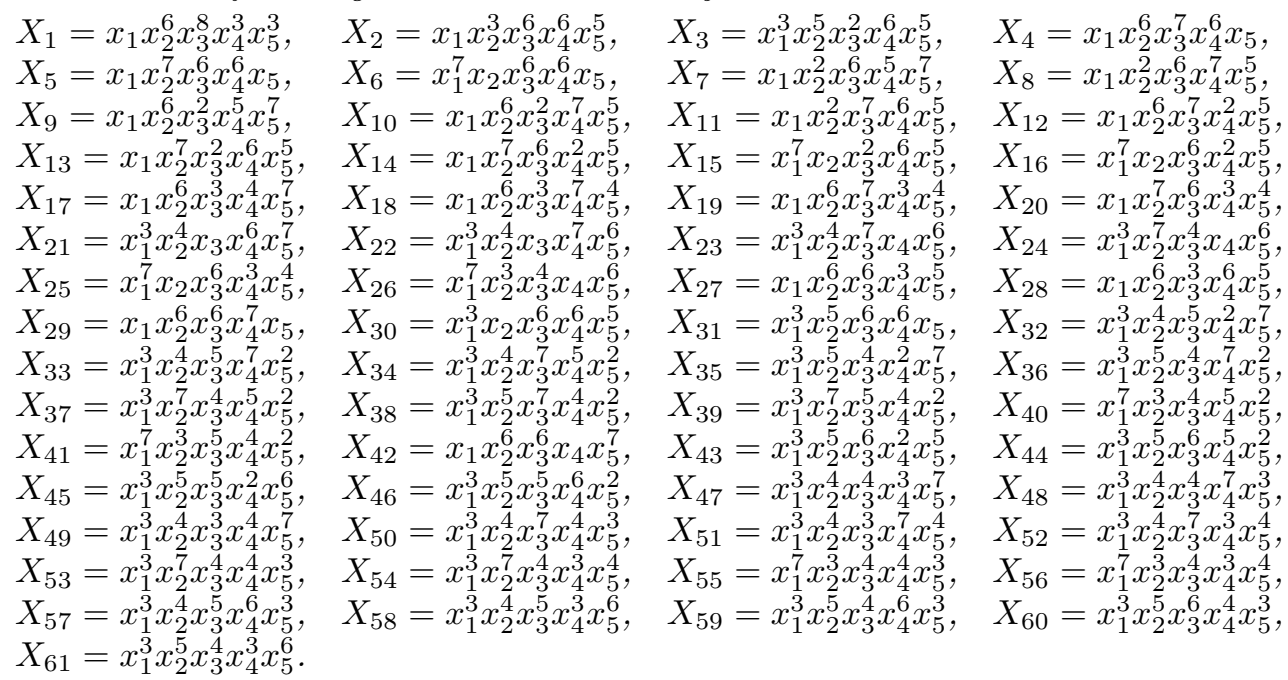

Proof. It is easily seen that $\omega\left(X_{1}\right)=(3,3,1,1)$ and $\omega\left(X_{j}\right)=(3,3,3)$ for $j=2,3, \ldots, 61$. We prove the lemma for the monomial $X_{1}=x_{1} x_{2}^{6} x_{3}^{8} x_{4}^{3} x_{5}^{3}$, and $X_{45}=x_{1}^{3} x_{2}^{5} x_{3}^{5} x_{4}^{2} x_{5}^{6}$. The others can be proven by a similar computation. By a direct computation, we have

$$
\begin{aligned}
X_{1}= & x_{1} x_{2}^{3} x_{3}^{3} x_{4}^{6} x_{5}^{8}+x_{1} x_{2}^{3} x_{3}^{3} x_{4}^{8} x_{5}^{6}+x_{1} x_{2}^{3} x_{3}^{6} x_{4}^{3} x_{5}^{8}+x_{1} x_{2}^{3} x_{3}^{6} x_{4}^{8} x_{5}^{3}+x_{1} x_{2}^{3} x_{3}^{8} x_{4}^{3} x_{5}^{6} \\
& +x_{1} x_{2}^{3} x_{3}^{8} x_{4}^{6} x_{5}^{3}+x_{1} x_{2}^{4} x_{3}^{3} x_{4}^{3} x_{5}^{10}+x_{1} x_{2}^{4} x_{3}^{3} x_{4}^{10} x_{5}^{3}+x_{1} x_{2}^{4} x_{3}^{10} x_{4}^{3} x_{5}^{3} \\
& +x_{1} x_{2}^{6} x_{3}^{3} x_{4}^{3} x_{5}^{8}+x_{1} x_{2}^{6} x_{3}^{3} x_{4}^{8} x_{5}^{3}+S q^{1}\left(A_{1}\right)+S q^{2}\left(A_{2}\right)+S q^{4}\left(A_{4}\right) \text { modulo }\left(\mathscr{P}_{5}^{-}(3,3,1,1)\right),
\end{aligned}
$$

where

$$
\begin{aligned}
A_{1}= & x_{1}^{2} x_{2}^{3} x_{3}^{5} x_{4}^{5} x_{5}^{5}+x_{1}^{2} x_{2}^{5} x_{3}^{3} x_{4}^{5} x_{5}^{5}+x_{1}^{2} x_{2}^{5} x_{3}^{5} x_{4}^{3} x_{5}^{5}+x_{1}^{2} x_{2}^{5} x_{3}^{5} x_{4}^{5} x_{5}^{3}, \\
A_{2}= & x_{1} x_{2}^{3} x_{3}^{3} x_{4}^{6} x_{5}^{6}+x_{1} x_{2}^{3} x_{3}^{5} x_{4}^{5} x_{5}^{5}+x_{1} x_{2}^{3} x_{3}^{6} x_{4}^{3} x_{5}^{6}+x_{1} x_{2}^{3} x_{3}^{6} x_{4}^{6} x_{5}^{3}+x_{1} x_{2}^{5} x_{3}^{3} x_{4}^{5} x_{5}^{5} \\
& +x_{1} x_{2}^{5} x_{3}^{5} x_{4}^{3} x_{5}^{5}+x_{1} x_{2}^{5} x_{3}^{5} x_{4}^{5} x_{5}^{3}+x_{1} x_{2}^{6} x_{3}^{3} x_{4}^{3} x_{5}^{6}+x_{1} x_{2}^{6} x_{3}^{3} x_{4}^{6} x_{5}^{3}+x_{1} x_{2}^{6} x_{3}^{6} x_{4}^{3} x_{5}^{3}, \\
A_{4}= & x_{1} x_{2}^{4} x_{3}^{3} x_{4}^{3} x_{5}^{6}+x_{1} x_{2}^{4} x_{3}^{3} x_{4}^{6} x_{5}^{3}+x_{1} x_{2}^{4} x_{3}^{6} x_{4}^{3} x_{5}^{3} .
\end{aligned}
$$

This relation implies that $X_{1}$ is strictly inadmissible. Next, an elementary calculation shows that

$$
X_{45}=S q^{1}\left(B_{1}\right)+S q^{2}\left(B_{2}\right)+S q^{4}\left(B_{4}\right)+x_{1}^{3} x_{2}^{3} x_{3}^{5} x_{4}^{4} x_{5}^{6}+x_{1}^{3} x_{2}^{5} x_{3}^{3} x_{4}^{4} x_{5}^{6} \text { modulo }\left(\mathscr{P}_{5}^{-}(3,3,3)\right),
$$

where

$$
B_{1}=x_{1}^{3} x_{2}^{3} x_{3}^{3} x_{4} x_{5}^{10}, \quad B_{2}=x_{1}^{5} x_{2}^{3} x_{3}^{3} x_{4}^{2} x_{5}^{6}, \quad \text { and } \quad B_{4}=x_{1}^{3} x_{2}^{3} x_{3}^{3} x_{4}^{2} x_{5}^{6} .
$$

The lemma is proved.

Now we denote by $\mathcal{C}$ the set of the following monomials:

$$
\begin{array}{llll}
x_{1}^{3} x_{2}^{12} x_{3} x_{4}^{2} x_{5}^{3} & x_{1}^{3} x_{2}^{12} x_{3} x_{4}^{3} x_{5}^{2}, & x_{1}^{3} x_{2}^{12} x_{3}^{3} x_{4} x_{5}^{2}, & x_{1}^{3} x_{2}^{4} x_{3} x_{4}^{2} x_{5}^{11}, \\
x_{1}^{3} x_{2}^{4} x_{3} x_{4}^{11} x_{5}^{2}, & x_{1}^{3} x_{2}^{4} x_{3}^{11} x_{4} x_{5}^{2}, & x_{1}^{7} x_{2}^{8} x_{3} x_{4}^{2} x_{5}^{3}, & x_{1}^{7} x_{2}^{8} x_{3} x_{4}^{3} x_{5}^{2}, \\
x_{1}^{7} x_{2}^{8} x_{3}^{3} x_{4} x_{5}^{2}, & x_{1}^{3} x_{2}^{4} x_{3} x_{4}^{3} x_{5}^{10}, & x_{1}^{3} x_{2}^{4} x_{3} x_{4}^{10} x_{5}^{3}, & x_{1}^{3} x_{2}^{4} x_{3}^{3} x_{4} x_{5}^{10}, \\
x_{1}^{3} x_{2}^{4} x_{3}^{3} x_{4}^{9} x_{5}^{2}, & x_{1}^{3} x_{2}^{4} x_{3}^{9} x_{4}^{2} x_{5}^{3}, & x_{1}^{3} x_{2}^{4} x_{3}^{9} x_{4}^{3} x_{5}^{2} . &
\end{array}
$$

A direct computation shows that $\bar{\Phi}^{+}\left(\mathscr{B}_{4}(3,3,1,1)\right) \cup \mathcal{C}$ is the set of 196 monomials: $y_{j}:=$ $y_{21, j}, 401 \leq j \leq 596$ (see Sect.6.3 of the online version [44].)

Proposition 3.3.6. Under the above notations, the $\mathbb{Z} / 2$-vector space $Q \mathscr{P}_{5}^{+}(3,3,1,1)$ is spanned by the set

$$
\left[\bar{\Phi}^{+}\left(\mathscr{B}_{4}(3,3,1,1)\right) \cup \mathcal{C}\right] .
$$

Proof. Let $X$ be an admissible monomial in $\mathscr{P}_{5}$ such that $\omega(X)=(3,3,1,1)$. Then $X=X_{(\{k, \ell\}, 5)} Y^{2}$ with $1 \leq k<\ell \leq 5$ and $Y$ a monomial of degree 9 in $\mathscr{P}_{5}$. Since $X$ is admissible, according to Theorem 2.2.1, $Y \in \mathscr{B}_{5}(3,1,1)$.

A direct computation shows that if $z \in \mathscr{B}_{5}^{+}(3,1,1), 1 \leq k<\ell \leq 5$, and $X_{(\{k, \ell\}, 5)} z^{2} \neq$ $y_{j}, \forall j, 401 \leq j \leq 596$ then there exists a monomial $w$ which is given in one of Lemmas 3.3.3- 3.3.5 such that $X_{(\{k, \ell\}, 5)} z^{2}=w z_{1}^{2^{a}}$ with a monomial $z_{1} \in \mathscr{P}_{5}$ and $a=\max \left\{m \in \mathbb{Z}: \omega_{m}(w)>0\right\}$. By Theorem 2.2.1, $X_{(\{k, \ell\}, 5)} z^{2}$ is inadmissible. Since $X=X_{(\{k, \ell\}, 5)} Y^{2}$ with $Y \in \mathscr{B}_{5}(3,1,1)$ and $X$ is admissible, one can see that $X=y_{j}, 401 \leq j \leq 596$. The lemma follows.

By a direct computation, we see that

$$
\bar{\Phi}^{+}\left(\mathscr{B}_{4}(3,3,3)\right) \cup\left\{x_{1}^{3} x_{2}^{4} x_{3} x_{4}^{5} x_{5}^{6}, x_{1}^{3} x_{2}^{5} x_{3} x_{4}^{6} x_{5}^{4}, x_{1}^{3} x_{2}^{5} x_{3}^{6} x_{4}^{3} x_{5}^{4}\right\}
$$

is the set consisting of 70 monomials: $y_{t}:=y_{21, t}, 597 \leq t \leq 666$ (see Sect.6.3 of the online version [44].) 
Proposition 3.3.7. The $\mathbb{Z} / 2$-vector space $Q \mathscr{P}_{5}^{+}(3,3,3)$ is spanned by the set $\left\{\left[y_{t}\right]: 597 \leq t \leq\right.$ $666\}$.

Proof. Let $u$ be an admissible monomial in $\mathscr{P}_{5}$ such that $\omega(u)=(3,3,3)$. Then $u=x_{i} x_{j} x_{\ell} y^{2}$ with $1 \leq i<j<\ell \leq 5$ and $y \in \mathscr{B}_{5}(3,3)$.

By a direct computation, we can verify that for any $X \in \mathscr{B}_{5}(3,3), 1 \leq i<j<\ell \leq 5$, such that $x_{i} x_{j} x_{\ell} X^{2} \neq y_{t}, \forall t, 597 \leq t \leq 666$, there is a monomial $z$ which is given in one of Lemmas 3.3.33.3.5 such that $x_{i} x_{j} x_{\ell} X^{2}=z w^{2^{b}}$ with suitable monomial $w \in \mathscr{P}_{5}$ and $b=\max \left\{r \in \mathbb{Z}: \omega_{r}(z)>0\right\}$. Then, according to Theorem 2.2.1, $x_{i} x_{j} x_{\ell} X^{2}$ nadmissible. Since $u=x_{i} x_{j} x_{\ell} y^{2}$ is admissible and $y \in \mathscr{B}_{5}(3,3)$, one gets $u=y_{t}$, for some $t$. This proves the proposition.

Proof of Proposition 3.3.1. From Propositions 3.3.6 and 3.3.7, the space $\operatorname{Ker}\left(\left(\widetilde{S q_{*}^{0}}\right)_{(5,21)}\right) \cap\left(Q \mathscr{P}_{5}^{+}\right)_{21}$ is spanned by the set $\left\{\left[y_{i}:=y_{21, i}\right]: 401 \leq i \leqslant 666\right\}$. Futhermore, this set is linearly independent in $\left(Q \mathscr{P}_{5}\right)_{21}$. Indeed, suppose there is a linear relation

$$
\mathcal{S}=\sum_{401 \leq i \leqslant 666} \gamma_{i} y_{i}=0 \operatorname{modulo}\left(\mathcal{A}_{2}^{+} \mathscr{P}_{5}\right),
$$

where $\gamma_{i} \in \mathbb{Z} / 2$. Based on Theorem 3.1.3 and Proposition 3.2.1, for $(k ; \mathscr{K}) \in \mathcal{N}_{5}$, we explicitly compute $\pi_{(k ; \mathscr{K})}(\mathcal{S})$ in terms of a given minimal set of $\mathcal{A}_{2}$-generators in $\mathscr{P}_{4}\left(\operatorname{modulo}\left(\mathcal{A}_{2}^{+} \mathscr{P}_{4}\right)\right)$. Computing directly from the relations $\pi_{(k ; p)}(\mathcal{S}) \equiv 0,1 \leq k<p \leq 5, \pi_{(1,(2 ; j))}(\mathcal{S}) \equiv 0, j=3,4,5$, and $\pi_{(1,(3 ; 4))}(\mathcal{S}) \equiv 0$, we obtain $\gamma_{i}=0, \forall i, 401 \leq i \leq 666$. This finishes the proof.

3.3.2. The case $t=2$. Note that $13.2^{2}-5=47$ and $\mu(47)=3<5$. Since Kameko's operation

$$
\left(\widetilde{S q_{*}^{0}}\right)_{(5,47)}:\left(Q \mathscr{P}_{5}\right)_{47} \rightarrow\left(Q \mathscr{P}_{5}\right)_{21}
$$

is an epimorphism of the $\mathbb{Z} / 2$-vector spaces, hence $\left(Q \mathscr{P}_{5}\right)_{47} \cong \operatorname{Ker}\left(\left(\widetilde{S q_{*}^{0}}\right)_{(5,47)}\right) \bigoplus\left(Q \mathscr{P}_{5}\right)_{21}$. This implies that we need only to determine $\operatorname{Ker}\left(\left(\widetilde{S q_{*}^{0}}\right)_{(5,47)}\right)$.

Remark 3.3.8. If $Y \in \mathscr{B}_{5}(47)$ and $[Y] \in \operatorname{Ker}\left(\left(\widetilde{S q_{*}^{0}}\right)_{(5,47)}\right)$, then $\omega_{1}(Y)=3$.

Indeed, we see that $z=x_{1}^{31} x_{2}^{15} x_{3}$ is the minimal spike of degree 47 in $\mathscr{P}_{5}$. By Proposition 3.1.2, $z \in \mathscr{B}_{5}(47)$. Since $[Y] \neq 0$, by Theorem 3.1.3, either $\omega_{1}(Y)=3$ or $\omega_{1}(Y)=5$. If $\omega_{1}(Y)=5$, then $Y=X_{(\emptyset, 5)} Z^{2}$ with $Z$ a monomial of degree 21 in $\mathscr{P}_{5}$. Since $Y$ is admissible, by Theorem 2.2.1, $Z \in \mathscr{B}_{5}(21)$. So, we have $\left(\widetilde{S q_{*}^{0}}\right)_{(5,47)}([Y])=[Z] \neq[0]$. This contradicts the face that $[Y] \in$ $\operatorname{Ker}\left(\left(\widetilde{S q_{*}^{0}}\right)_{(5,47)}\right)$; hence we get $\omega_{1}(Y)=3$.

From Remark 3.3.8, we have $Y=x_{k} x_{\ell} x_{m} g^{2}$ with $1 \leq k<\ell<m \leq 5$ and $g \in \mathscr{B}_{5}(22)$. Thus, to determine $\operatorname{Ker}\left(\left(\widetilde{S q_{*}^{0}}\right)_{(5,47)}\right)$, we need to compute all the admissible monomials of degree 22 in the $\mathcal{A}_{2}$-module $\mathscr{P}_{5}$.

\section{Computation of $\left(Q \mathscr{P}_{5}\right)_{22}$}

We consider the following weight vectors:

$$
\omega_{(1)}=(2,2,2,1), \omega_{(2)}=(2,4,1,1), \omega_{(3)}=(2,4,3), \omega_{(4)}=(4,3,1,1), \omega_{(5)}=(4,3,3) .
$$

It is easy to see that $\operatorname{deg} \omega_{(i)}=22,1 \leq i \leq 5$. By Proposition $3.1 .2, x_{1}^{15} x_{2}^{7}$ is the minimal spike in $\mathscr{B}_{5}(22)$ and $\omega\left(x_{1}^{15} x_{2}^{7}\right)=\omega_{(1)}$. Let $u$ be an admissible monomial of degree 22 in $\mathscr{P}_{5}$. Then $[u] \neq[0]$ and by Theorem 3.1.3, either $\omega_{1}(u)=2$ or $\omega_{1}(u)=4$. Since $u \in \mathscr{B}_{5}(22)$, by Theorem 2.2.1, if $\omega_{1}(u)=2$, then $u=X_{(\{i, j, k\}, 5)} y^{2}$ with $y \in \mathscr{B}_{5}(10)$ and $1 \leqslant i<j<k \leq 5$. According to Tín [69], $\omega(y)$ is one of the sequences $(2,2,1),(4,1,1)$, and $(4,3)$. If $\omega_{1}(u)=4$, then $u=X_{(\{i\}, 5)} y_{1}^{2}$ with $y_{1}$ a monomial of degree 9 in $\mathscr{P}_{5}$ and $1 \leq i \leq 5$. By Tín [69], either $\omega\left(y_{1}\right)=(3,1,1)$ or $\omega\left(y_{1}\right)=(3,3)$. Hence, we have the following.

Remark 3.3.9. If $u \in \mathscr{B}_{5}(22)$, then $\omega(u)$ is one of the sequences $\omega_{(t)}, 1 \leq t \leq 5$.

As it is known, $\left(Q \mathscr{P}_{5}\right)_{22}=\left(Q \mathscr{P}_{5}^{0}\right)_{22} \bigoplus\left(Q \mathscr{P}_{5}^{+}\right)_{22}$. By Sum [62], $Q \mathscr{P}_{5}^{+}$has dimension 72 in degree 22. Then, based on the rerults in [37] and [21] with fact that $\left(Q \mathscr{P}_{5}^{0}\right)_{22}=\bigoplus_{1 \leq s \leq 4} \bigoplus_{1 \leq u \leq\left(\begin{array}{l}5 \\ u\end{array}\right)}\left(Q \mathscr{P}_{s}^{+}\right)_{22}$, we deduce that $\operatorname{dim}\left(Q \mathscr{P}_{5}^{0}\right)_{22}=\left(\begin{array}{l}5 \\ 2\end{array}\right) .2+\left(\begin{array}{l}5 \\ 3\end{array}\right) .8+\left(\begin{array}{l}5 \\ 4\end{array}\right) \cdot 72=460$, and that

$$
\mathscr{B}_{5}^{0}(22)=\bar{\Phi}^{0}\left(\mathscr{B}_{4}(22)\right)=\left\{y_{22, t}: 1 \leq t \leq 460\right\},
$$

where the monomials $y_{22, t}, 1 \leq t \leq 460$, are determined in Sect.6.4 of the online version [44].

Next, we compute $\left(Q \mathscr{P}_{5}^{+}\right)_{22}$. For $r, k \in \mathbb{N}$ and $1 \leq k \leq 5$, we denote

$$
\overline{\mathscr{B}}(k, 22):=\left\{x_{k}^{2^{r}-1} \rho_{(k, 5)}(x) \in\left(\mathscr{P}_{5}\right)_{22}: x=\prod_{1 \leq i \leq 4} x_{i}^{a_{i}} \in \mathscr{B}_{4}\left(23-2^{r}\right), \alpha\left(27-2^{r}\right) \leq 4\right\} .
$$


By Mothebe and Uys [28], $\overline{\mathscr{B}}(5,22) \subseteq \mathscr{B}_{5}(22), 1 \leq k \leq 5$. We set

$$
\overline{\mathscr{B}}\left(k, \omega_{(t)}\right):=\overline{\mathscr{B}}(k, 22) \cap \mathscr{P}_{5}\left(\omega_{(t)}\right), \overline{\mathscr{B}}^{+}\left(k, \omega_{(t)}\right):=\overline{\mathscr{B}}\left(k, \omega_{(t)}\right) \cap\left(\mathscr{P}_{5}^{+}\right)_{22},
$$

for all $1 \leq t, k \leq 5$. By a simple computation, we find that

$$
\bar{\Phi}^{+}\left(\mathscr{B}_{4}\left(\omega_{(1)}\right) \bigcup\left(\bigcup_{1 \leq k \leq 5} \overline{\mathscr{B}}^{+}\left(k, \omega_{(1)}\right)\right)\right.
$$

is the set of 31 admissible monomials: $y_{22, i}, 461 \leq i \leq 491$ (see Sect.6.5 of the online version [44]).

Denote by $\mathcal{D}$ is the set of the following monomials:

$$
\begin{array}{lll}
y_{22,492}=x_{1} x_{2} x_{3}^{6} x_{4}^{6} x_{5}^{8}, & y_{22,493}=x_{1} x_{2} x_{3}^{6} x_{4}^{10} x_{5}^{4}, & y_{22,494}=x_{1} x_{2}^{2} x_{3}^{3} x_{4}^{4} x_{5}^{12}, \\
y_{22,495}=x_{1} x_{2}^{2} x_{3}^{3} x_{4}^{12} x_{5}^{4}, & y_{22,496}=x_{1} x_{2}^{2} x_{3}^{4} x_{4}^{9} x_{5}^{6}, & y_{22,497}=x_{1} x_{2}^{2} x_{3}^{5} x_{4}^{8} x_{5}^{6}, \\
y_{22,498}=x_{1} x_{2}^{3} x_{3}^{2} x_{4}^{4} x_{5}^{12}, & y_{22,499}=x_{1} x_{2}^{3} x_{3}^{2} x_{4}^{12} x_{5}^{4}, & y_{22,500}=x_{1} x_{2}^{3} x_{3}^{4} x_{4}^{8} x_{5}^{6}, \\
y_{22,501}=x_{1} x_{2}^{3} x_{3}^{6} x_{4}^{4} x_{5}^{8}, & y_{22,502}=x_{1} x_{2}^{3} x_{3}^{6} x_{4}^{8} x_{5}^{4}, & y_{22,503}=x_{1}^{3} x_{2} x_{3}^{2} x_{4}^{4} x_{5}^{12}, \\
y_{22,504}=x_{1}^{3} x_{2} x_{3}^{2} x_{4}^{12} x_{5}^{4}, & y_{22,505}=x_{1}^{3} x_{2} x_{3}^{4} x_{4}^{8} x_{5}^{6}, & y_{22,506}=x_{1}^{3} x_{2} x_{3}^{6} x_{4}^{4} x_{5}^{8}, \\
y_{22,507}=x_{1}^{3} x_{2} x_{3}^{6} x_{4}^{8} x_{5}^{4}, & y_{22,508}=x_{1}^{3} x_{2}^{5} x_{3}^{2} x_{4}^{4} x_{5}^{8}, & y_{22,509}=x_{1}^{3} x_{2}^{5} x_{3}^{2} x_{4}^{8} x_{5}^{4}, \\
y_{22,510}=x_{1}^{3} x_{2}^{5} x_{3}^{8} x_{4}^{2} x_{5}^{4} . & &
\end{array}
$$

Proposition 3.3.10. $\mathscr{B}_{5}^{+}\left(\omega_{(1)}\right)=\bar{\Phi}^{+}\left(\mathscr{B}_{4}\left(\omega_{(1)}\right) \bigcup\left(\bigcup_{1 \leq k \leq 5} \overline{\mathscr{B}}^{+}\left(k, \omega_{(1)}\right)\right) \bigcup \mathcal{D}\right.$.

In order to prove the proposition, we need some lemmas.

Lemma 3.3.11. The following monomials are strictly inadmissible:

(i) $x_{1}^{2} x_{j} x_{k}^{2} x_{l}^{3} x_{m}^{6}, x_{1}^{6} x_{j} x_{k} x_{l}^{2} x_{m}^{4}, l<m, x_{1}^{2} x_{j} x_{k} x_{l}^{4} x_{m}^{6}$.

Here $(j, k, l, m)$ is a permutation of $(2,3,4,5)$;

(ii) $\quad x_{1} x_{2}^{2} x_{3}^{4} x_{4}^{6} x_{5}, \quad x_{1} x_{2}^{2} x_{3}^{6} x_{4} x_{5}^{4}, \quad x_{1} x_{2}^{2} x_{3}^{6} x_{4}^{4} x_{5}, \quad x_{1} x_{2}^{6} x_{3}^{2} x_{4} x_{5}^{4}$, $x_{1} x_{2}^{6} x_{3}^{2} x_{4}^{4} x_{5}, \quad x_{1}^{3} x_{2}^{3} x_{3}^{4} x_{4}^{2} x_{5}^{2}, \quad x_{1}^{3} x_{2}^{4} x_{3}^{2} x_{4}^{2} x_{5}^{3}, \quad x_{1}^{3} x_{2}^{4} x_{3}^{2} x_{4}^{3} x_{5}^{2}$, $x_{1}^{3} x_{2}^{4} x_{3}^{3} x_{4}^{2} x_{5}^{2}, \quad x_{1}^{3} x_{2}^{2} x_{3} x_{4}^{2} x_{5}^{6}, \quad x_{1}^{3} x_{2}^{2} x_{3} x_{4}^{6} x_{5}^{2}, \quad x_{1}^{3} x_{2}^{2} x_{3}^{2} x_{4} x_{5}^{6}$, $x_{1}^{3} x_{2}^{2} x_{3}^{2} x_{4}^{6} x_{5}, \quad x_{1}^{3} x_{2}^{2} x_{3}^{6} x_{4} x_{5}^{2}, \quad x_{1}^{3} x_{2}^{2} x_{3}^{6} x_{4}^{2} x_{5}, \quad x_{1}^{3} x_{2}^{6} x_{3} x_{4}^{2} x_{5}^{2}$, $x_{1}^{3} x_{2}^{6} x_{3}^{2} x_{4} x_{5}^{2}, \quad x_{1}^{3} x_{2}^{6} x_{3}^{2} x_{4}^{2} x_{5}, \quad x_{1}^{2} x_{2}^{3} x_{3}^{3} x_{4}^{3} x_{5}^{3}, \quad x_{1}^{3} x_{2}^{2} x_{3}^{3} x_{4}^{3} x_{5}^{3}$, $x_{1}^{3} x_{2}^{3} x_{3}^{2} x_{4}^{3} x_{5}^{3}, \quad x_{1}^{3} x_{2}^{3} x_{3}^{3} x_{4}^{2} x_{5}^{3}, \quad x_{1}^{3} x_{2}^{3} x_{3}^{3} x_{4}^{3} x_{5}^{2}$.

Proof. We prove the lemma for the monomials $u=x_{1}^{2} x_{j} x_{k}^{2} x_{l}^{3} x_{m}^{6}$, and $v=x_{1} x_{2}^{2} x_{3}^{4} x_{4}^{6} x_{5}$. The others can be proved by a similar computation. We have $\omega(u)=(2,4,1)$ and $\omega(v)=(2,2,2)$. By a simple computation, one gets

$$
\begin{aligned}
u & =x_{1} x_{j}^{2} x_{k}^{2} x_{l}^{3} x_{m}^{6}+S q^{1}\left(x_{1} x_{j} x_{k}^{2} x_{l}^{3} x_{m}^{6}\right) \operatorname{modulo}\left(\mathscr{P}_{5}^{-}(2,4,1)\right), \\
v & =x_{1} x_{2} x_{3}^{4} x_{4}^{6} x_{5}^{2}+x_{1} x_{2}^{2} x_{3}^{4} x_{4}^{5} x_{5}^{2}+S q^{1}\left(f_{1}\right)+S q^{2}\left(f_{2}\right) \operatorname{modulo}\left(\mathscr{P}_{5}^{-}(2,2,2)\right),
\end{aligned}
$$

where $f_{1}=x_{1}^{2} x_{2} x_{3}^{4} x_{4}^{5} x_{5}$ and $f_{2}=x_{1} x_{2} x_{3}^{4} x_{4}^{5} x_{5}$. Hence, $u$ and $v$ are strictly inadmissible. The lemma follows.

The following lemma can easily be proved by a direct computation.

Lemma 3.3.12. If $(i, j, k, l, m)$ is a permutation of $(1,2,3,4,5)$, then the following monomials are strictly inadmissible:

(i) $x_{i}^{6} x_{j} x_{k}^{7}, x_{i}^{2} x_{j}^{5} x_{k}^{7}, x_{i}^{3} x_{j}^{4} x_{k}^{7}, x_{i}^{2} x_{j} x_{k}^{2} x_{l}^{2} x_{m}^{7}$, $x_{i}^{2} x_{j}^{5} x_{k}^{2} x_{l}^{2} x_{m}^{3}, x_{i}^{2} x_{j}^{4} x_{k}^{2} x_{l}^{3} x_{m}^{3}, x_{i}^{2} x_{j} x_{k}^{2} x_{l}^{2} x_{m}^{3}, i<j$

(ii) $x_{i} x_{j}^{6} x_{k}^{3} x_{l}^{2} x_{m}^{2}, j<k, x_{i}^{3} x_{j}^{6} x_{k}^{5}, x_{i}^{6} x_{j}^{3} x_{k}^{5}, x_{i}^{2} x_{j} x_{k} x_{l}^{3} x_{m}^{3}, x_{i}^{2} x_{j} x_{k} x_{l}^{2}, i<j<k$;

(iii) $x_{i}^{2} x_{j} x_{k}^{4} x_{l}^{3} x_{m}^{4}, x_{i}^{2} x_{j}^{4} x_{k} x_{l}^{3} x_{m}^{4}, i<j<k, l<m$.

Lemma 3.3.13. The following monomials are strictly inadmissible:

$\begin{array}{llll}x_{1} x_{2}^{2} x_{3}^{2} x_{4}^{7} x_{5}^{10}, & x_{1} x_{2}^{2} x_{3}^{4} x_{4}^{3} x_{5}^{12}, & x_{1} x_{2}^{2} x_{3}^{4} x_{4}^{11} x_{5}^{4}, & x_{1} x_{2}^{2} x_{3}^{7} x_{4}^{2} x_{5}^{10}, \\ x_{1} x_{2}^{2} x_{3}^{7} x_{4}^{8} x_{5}^{4}, & x_{1} x_{2}^{2} x_{3}^{7} x_{4}^{10} x_{5}^{2}, & x_{1} x_{2}^{2} x_{3}^{12} x_{4}^{3} x_{5}^{4}, & x_{1} x_{2}^{6} x_{3} x_{4}^{6} x_{5}^{8}, \\ x_{1} x_{2}^{6} x_{3} x_{4}^{10} x_{5}^{4}, & x_{1} x_{2}^{6} x_{3}^{3} x_{4}^{6} x_{5}^{6}, & x_{1} x_{2}^{6} x_{3}^{6} x_{4}^{3} x_{5}^{6}, & x_{1} x_{2}^{6} x_{3}^{6} x_{4}^{6} x_{5}^{3}, \\ x_{1} x_{2}^{6} x_{3}^{9} x_{4}^{2} x_{5}^{4}, & x_{1} x_{2}^{7} x_{3}^{2} x_{4}^{2} x_{5}^{10}, & x_{1} x_{2}^{7} x_{3}^{2} x_{4}^{8} x_{5}^{4}, & x_{1} x_{2}^{7} x_{3}^{2} x_{4}^{10} x_{5}^{2}, \\ x_{1} x_{2}^{7} x_{3}^{8} x_{4}^{2} x_{5}^{4}, & x_{1} x_{2}^{7} x_{3}^{10} x_{4}^{2} x_{5}^{2}, & x_{1}^{3} x_{2}^{3} x_{3}^{4} x_{4}^{4} x_{5}^{8}, & x_{1}^{3} x_{2}^{3} x_{3}^{4} x_{4}^{8} x_{5}^{4}, \\ x_{1}^{3} x_{2}^{4} x_{3} x_{4}^{2} x_{5}^{12}, & x_{1}^{3} x_{2}^{4} x_{3} x_{4}^{10} x_{5}^{4}, & x_{1}^{3} x_{2}^{4} x_{3}^{4} x_{4}^{4} x_{5}^{7}, & x_{1}^{3} x_{2}^{4} x_{3}^{4} x_{4}^{5} x_{5}^{6}, \\ x_{1}^{3} x_{2}^{4} x_{3}^{4} x_{4}^{7} x_{5}^{4}, & x_{1}^{3} x_{2}^{4} x_{3}^{5} x_{4}^{4} x_{5}^{6}, & x_{1}^{3} x_{2}^{4} x_{3}^{5} x_{4}^{6} x_{5}^{4}, & x_{1}^{3} x_{2}^{4} x_{3}^{7} x_{4}^{4} x_{5}^{4}, \\ x_{1}^{3} x_{2}^{4} x_{3}^{9} x_{4}^{2} x_{5}^{4}, & x_{1}^{3} x_{2}^{5} x_{3}^{4} x_{4}^{4} x_{5}^{6}, & x_{1}^{3} x_{2}^{5} x_{3}^{4} x_{4}^{6} x_{5}^{4}, & x_{1}^{3} x_{2}^{5} x_{3}^{6} x_{4}^{4} x_{5}^{4}, \\ x_{1}^{3} x_{2}^{7} x_{3}^{4} x_{4}^{4} x_{5}^{4}, & x_{1}^{3} x_{2}^{12} x_{3} x_{4}^{2} x_{5}^{4}, & x_{1}^{7} x_{2} x_{3}^{2} x_{4}^{2} x_{5}^{10}, & x_{1}^{7} x_{2} x_{3}^{2} x_{4}^{8} x_{5}^{4}, \\ x_{1}^{7} x_{2} x_{3}^{2} x_{4}^{10} x_{5}^{2}, & x_{1}^{7} x_{2} x_{3}^{8} x_{4}^{2} x_{5}^{4}, & x_{1}^{7} x_{2} x_{3}^{10} x_{4}^{2} x_{5}^{2}, & x_{1}^{7} x_{2}^{3} x_{3}^{4} x_{4}^{4} x_{5}^{4}, \\ x_{1}^{7} x_{2}^{8} x_{3} x_{4}^{2} x_{5}^{4}, & x_{1}^{7} x_{2}^{9} x_{3}^{2} x_{4}^{2} x_{5}^{2} . & & \end{array}$

Proof. We prove the lemma for the monomials $x=x_{1} x_{2}^{2} x_{3}^{4} x_{4}^{3} x_{5}^{12}$, and $y=x_{1}^{3} x_{2}^{3} x_{3}^{4} x_{4}^{4} x_{5}^{8}$. The others can be proved by a similar computation. By a direct computation using the Cartan formula, we have

$$
\begin{aligned}
x= & x_{1} x_{2}^{2} x_{3}^{3} x_{4}^{4} x_{5}^{12}+x_{1} x_{2}^{2} x_{3}^{4} x_{4}^{2} x_{5}^{13}+x_{1} x_{2}^{2} x_{3}^{2} x_{4}^{4} x_{5}^{13}+x_{1} x_{2} x_{3}^{6} x_{4}^{6} x_{5}^{8}+x_{1} x_{2} x_{3}^{6} x_{4}^{4} x_{5}^{10} \\
& +x_{1} x_{2} x_{3}^{4} x_{4}^{6} x_{5}^{10}+S q^{1}\left(f_{1}\right)+S q^{2}\left(f_{2}\right)+S q^{4}\left(f_{4}\right) \text { modulo }\left(\mathscr{P}_{5}^{-}\left(\omega_{(1)}\right)\right)
\end{aligned}
$$


where

$$
\begin{aligned}
f_{1}= & x_{1} x_{2}^{4} x_{3}^{3} x_{4}^{4} x_{5}^{9}+x_{1} x_{2}^{4} x_{3}^{3} x_{4}^{5} x_{5}^{8}+x_{1} x_{2}^{4} x_{3}^{4} x_{4}^{3} x_{5}^{9}+x_{1} x_{2}^{4} x_{3}^{4} x_{4}^{5} x_{5}^{7} \\
& +x_{1} x_{2}^{4} x_{3}^{5} x_{4}^{3} x_{5}^{8}+x_{1} x_{2}^{4} x_{3}^{5} x_{4}^{4} x_{5}^{7}+x_{1}^{2} x_{2} x_{3}^{4} x_{4}^{5} x_{5}^{9}+x_{1}^{2} x_{2} x_{3}^{5} x_{4}^{4} x_{5}^{9} \\
& +x_{1}^{2} x_{2} x_{3}^{5} x_{4}^{5} x_{5}^{8}+x_{1}^{4} x_{2}^{4} x_{3}^{3} x_{4}^{3} x_{5}^{7}, \\
f_{2}= & x_{1} x_{2} x_{3}^{4} x_{4}^{5} x_{5}^{9}+x_{1} x_{2} x_{3}^{5} x_{4}^{4} x_{5}^{9}+x_{1} x_{2} x_{3}^{5} x_{4}^{5} x_{5}^{8}+x_{1} x_{2}^{2} x_{3}^{3} x_{4}^{4} x_{5}^{10} \\
& +x_{1} x_{2}^{2} x_{3}^{3} x_{4}^{6} x_{5}^{8}+x_{1} x_{2}^{2} x_{3}^{4} x_{4}^{3} x_{5}^{10}+x_{1} x_{2}^{2} x_{3}^{4} x_{4}^{6} x_{5}^{7}+x_{1} x_{2}^{2} x_{3}^{6} x_{4}^{3} x_{5}^{8} \\
& +x_{1} x_{2}^{2} x_{3}^{6} x_{4}^{4} x_{5}^{7}+x_{1} x_{2}^{4} x_{3}^{2} x_{4}^{2} x_{5}^{11}+x_{1}^{2} x_{2}^{4} x_{3}^{3} x_{4}^{3} x_{5}^{8}+x_{1}^{2} x_{2}^{4} x_{3}^{3} x_{4}^{4} x_{5}^{7} \\
& +x_{1}^{2} x_{2}^{4} x_{3}^{4} x_{4}^{3} x_{5}^{7}, \\
f_{4}= & x_{1} x_{2}^{2} x_{3}^{2} x_{4}^{2} x_{5}^{11}+x_{1} x_{2}^{2} x_{3}^{4} x_{4}^{4} x_{5}^{7} .
\end{aligned}
$$

The above equalities show that $x$ is strictly inadmissible. By a similar computation, we obtain

$$
\begin{aligned}
& y=x_{1}^{2} x_{2} x_{3}^{2} x_{4}^{13} x_{5}^{4}+x_{1}^{2} x_{2} x_{3}^{3} x_{4}^{12} x_{5}^{4}+x_{1}^{2} x_{2} x_{3}^{4} x_{4}^{7} x_{5}^{8}+x_{1}^{2} x_{2} x_{3}^{4} x_{4}^{13} x_{5}^{2}+x_{1}^{2} x_{2} x_{3}^{5} x_{4}^{6} x_{5}^{8} \\
& +x_{1}^{2} x_{2} x_{3}^{6} x_{4}^{9} x_{5}^{4}+x_{1}^{2} x_{2} x_{3}^{8} x_{4}^{7} x_{5}^{4}+x_{1}^{2} x_{2} x_{3}^{12} x_{4}^{5} x_{5}^{2}+x_{1}^{2} x_{2}^{3} x_{3}^{5} x_{4}^{8} x_{5}^{4}+x_{1}^{2} x_{2}^{3} x_{3}^{8} x_{4}^{5} x_{5}^{4} \\
& +x_{1}^{2} x_{2}^{5} x_{3}^{2} x_{4}^{5} x_{5}^{8}+x_{1}^{2} x_{2}^{5} x_{3}^{2} x_{4}^{9} x_{5}^{4}+x_{1}^{2} x_{2}^{5} x_{3}^{3} x_{4}^{8} x_{5}^{4}+x_{1}^{2} x_{2}^{5} x_{3}^{4} x_{4}^{3} x_{5}^{8}+x_{1}^{2} x_{2}^{5} x_{3}^{4} x_{4}^{9} x_{5}^{2} \\
& +x_{1}^{2} x_{2}^{5} x_{3}^{8} x_{4}^{5} x_{5}^{2}+x_{1}^{3} x_{2} x_{3}^{2} x_{4}^{12} x_{5}^{4}+x_{1}^{3} x_{2} x_{3}^{4} x_{4}^{6} x_{5}^{8}+x_{1}^{3} x_{2} x_{3}^{4} x_{4}^{10} x_{5}^{4}+x_{1}^{3} x_{2} x_{3}^{4} x_{4}^{12} x_{5}^{2} \\
& +x_{1}^{3} x_{2} x_{3}^{4} x_{4}^{12} x_{5}^{2}+x_{1}^{3} x_{2}^{2} x_{3}^{4} x_{4}^{9} x_{5}^{4}+x_{1}^{3} x_{2}^{2} x_{3}^{8} x_{4}^{5} x_{5}^{4} \\
& +S q^{1}\left(g_{1}\right)+S q^{2}\left(g_{2}\right)+S q^{4}\left(g_{4}\right)+S q^{8}\left(x_{1}^{3} x_{2}^{3} x_{3}^{2} x_{4}^{4} x_{5}^{2}\right) \text { modulo }\left(\mathscr{P}_{5}^{-}\left(\omega_{(1)}\right)\right) \text {, where } \\
& g_{1}=x_{1}^{3} x_{2}^{3} x_{3}^{2} x_{4}^{9} x_{5}^{4}+x_{1}^{3} x_{2}^{3} x_{3}^{4} x_{4}^{9} x_{5}^{2}+x_{1}^{3} x_{2}^{3} x_{3}^{2} x_{4}^{5} x_{5}^{8}+x_{1}^{3} x_{2}^{3} x_{3}^{8} x_{4}^{5} x_{5}^{2}+x_{1}^{5} x_{2} x_{3}^{2} x_{4}^{9} x_{5}^{4} \\
& +x_{1}^{3} x_{2} x_{3}^{4} x_{4}^{9} x_{5}^{4}+x_{1}^{5} x_{2} x_{3}^{4} x_{4}^{9} x_{5}^{2}+x_{1}^{5} x_{2} x_{3}^{8} x_{4}^{5} x_{5}^{2}+x_{1}^{3} x_{2} x_{3}^{8} x_{4}^{5} x_{5}^{4}+x_{1}^{5} x_{2} x_{3}^{6} x_{4}^{5} x_{5}^{4} \\
& +x_{1}^{3} x_{2}^{4} x_{3}^{5} x_{4}^{5} x_{5}^{4}+x_{1}^{5} x_{2} x_{3}^{5} x_{4}^{6} x_{5}^{4}+x_{1}^{5} x_{2} x_{3}^{3} x_{4}^{8} x_{5}^{4}+x_{1}^{3} x_{2}^{3} x_{3}^{3} x_{4}^{8} x_{5}^{4}+x_{1}^{5} x_{2} x_{3}^{2} x_{4}^{5} x_{5}^{8} \\
& +x_{1}^{5} x_{2} x_{3}^{4} x_{4}^{3} x_{5}^{8}+x_{1}^{3} x_{2}^{3} x_{3}^{4} x_{4}^{3} x_{5}^{8}, \\
& g_{2}=x_{1}^{5} x_{2}^{3} x_{3}^{2} x_{4}^{8} x_{5}^{2}+x_{1}^{5} x_{2}^{3} x_{3}^{2} x_{4}^{6} x_{5}^{4}+x_{1}^{5} x_{2}^{3} x_{3}^{4} x_{4}^{6} x_{5}^{2}+x_{1}^{2} x_{2}^{3} x_{3}^{2} x_{4}^{9} x_{5}^{4}+x_{1}^{3} x_{2}^{2} x_{3}^{2} x_{4}^{9} x_{5}^{4} \\
& +x_{1}^{3} x_{2} x_{3}^{2} x_{4}^{10} x_{5}^{4}+x_{1}^{6} x_{2} x_{3}^{2} x_{4}^{7} x_{5}^{4}+x_{1}^{2} x_{2}^{3} x_{3}^{4} x_{4}^{9} x_{5}^{2}+x_{1}^{3} x_{2}^{2} x_{3}^{4} x_{4}^{9} x_{5}^{2}+x_{1}^{3} x_{2} x_{3}^{4} x_{4}^{10} x_{5}^{2} \\
& +x_{1}^{6} x_{2} x_{3}^{4} x_{4}^{7} x_{5}^{2}+x_{1}^{2} x_{2}^{3} x_{3}^{8} x_{4}^{5} x_{5}^{2}+x_{1}^{2} x_{2}^{3} x_{3}^{2} x_{4}^{5} x_{5}^{8}+x_{1}^{3} x_{2}^{2} x_{3}^{8} x_{4}^{5} x_{5}^{2}+x_{1}^{3} x_{2}^{2} x_{3}^{2} x_{4}^{5} x_{5}^{8} \\
& +x_{1}^{3} x_{2} x_{3}^{8} x_{4}^{6} x_{5}^{2}+x_{1}^{6} x_{2} x_{3}^{6} x_{4}^{5} x_{5}^{2}+x_{1}^{3} x_{2}^{2} x_{3}^{6} x_{4}^{5} x_{5}^{4}+x_{1}^{3} x_{2}^{2} x_{3}^{5} x_{4}^{6} x_{5}^{4}+x_{1}^{3} x_{2}^{2} x_{3}^{3} x_{4}^{8} x_{5}^{4} \\
& +x_{1}^{2} x_{2}^{3} x_{3}^{3} x_{4}^{8} x_{5}^{4}+x_{1}^{6} x_{2} x_{3}^{2} x_{4}^{3} x_{5}^{8}+x_{1}^{3} x_{2} x_{3}^{2} x_{4}^{6} x_{5}^{8}+x_{1}^{3} x_{2}^{2} x_{3}^{4} x_{4}^{3} x_{5}^{8}+x_{1}^{6} x_{2} x_{3}^{3} x_{4}^{6} x_{5}^{4} \\
& +x_{1}^{2} x_{2}^{3} x_{3}^{4} x_{4}^{3} x_{5}^{8}+x_{1}^{2} x_{2} x_{3}^{10} x_{4}^{5} x_{5}^{2}+x_{1}^{2} x_{2} x_{3}^{2} x_{4}^{7} x_{5}^{8}+x_{1}^{2} x_{2} x_{3}^{2} x_{4}^{11} x_{5}^{4}+x_{1}^{2} x_{2} x_{3}^{3} x_{4}^{6} x_{5}^{8} \\
& +x_{1}^{2} x_{2} x_{3}^{3} x_{4}^{10} x_{5}^{4}+x_{1}^{2} x_{2} x_{3}^{4} x_{4}^{11} x_{5}^{2}+x_{1}^{2} x_{2} x_{3}^{6} x_{4}^{9} x_{5}^{2}+x_{1}^{2} x_{2} x_{3}^{8} x_{4}^{7} x_{5}^{2} \text {, } \\
& g_{4}=x_{1}^{3} x_{2}^{3} x_{3}^{2} x_{4}^{6} x_{5}^{4}+x_{1}^{3} x_{2}^{3} x_{3}^{4} x_{4}^{6} x_{5}^{2}+x_{1}^{4} x_{2} x_{3}^{2} x_{4}^{7} x_{5}^{4} \\
& +x_{1}^{4} x_{2} x_{3}^{4} x_{4}^{7} x_{5}^{2}+x_{1}^{4} x_{2} x_{3}^{6} x_{4}^{5} x_{5}^{2}+x_{1}^{4} x_{2} x_{3}^{3} x_{4}^{6} x_{5}^{4} \text {. }
\end{aligned}
$$

The above relations imply that $y$ is also strictly inadmissible. The lemma is proved.

Proof of Proposition 3.3.10. We denote by $y_{t}:=y_{22, t}, 461 \leq t \leq 510$ the admissible monomials in $\mathscr{B}_{5}^{+}\left(\omega_{(1)}\right)$ (see Sect.6.5 of the online version [44].) For $x \in \mathscr{B}_{5}^{+}\left(\omega_{(1)}\right)$, we have $x=X_{\{i, j, k\}} y^{2}$ with $y$ a monomial of degree 10 in $\mathscr{P}_{5}$, and $1 \leq i<j<k \leq 5$. Since $x$ is admissible, by Theorem 2.2.1, $y \in \mathscr{B}_{5}(2,2,1)$.

Let $y_{1} \in \mathscr{B}_{5}(2,2,1)$ such that $X_{(\{i, j, k\}, 5)} y_{1}^{2} \in \mathscr{P}_{5}^{+}$. By a direct computation using a result in [69], we see that if $X_{(\{i, j, k\}, 5)} y_{1}^{2} \neq y_{t}$, for all $t, 461 \leq t \leq 510$, then there is a monomial $w$ which is given in one of Lemmas 3.3.12 - 3.3.13 such that $X_{(\{i, j, k\}, 5)} y_{1}^{2}=w z^{2^{u}}$ with suitable monomial $z \in \mathscr{P}_{5}$ and $u=\max \left\{j \in \mathbb{Z}: \omega_{j}(w)>0\right\}$. By Theorem 2.2.1, $X_{(\{i, j, k\}, 5)} y_{1}^{2}$ is inadmissible. Since $x=X_{(\{i, j, k\}, 5)} y^{2}$ and $x$ is admissible, one gets $x=y_{t}$. This implies $Q \mathscr{P}_{5}^{+}\left(\omega_{(1)}\right)$ is spanned by the set $\left\{\left[y_{t}:=y_{22, t}\right]_{\omega_{(1)}}: 461 \leq t \leq 510\right\}$.

We now prove the set $\left\{\left[y_{t}\right]_{\omega_{(1)}}: 461 \leq t \leq 510\right\}$ is linearly independent in $Q \mathscr{P}_{5}\left(\omega_{(1)}\right)$. Suppose there is a linear relation

$$
\mathcal{S}=\sum_{461 \leq t \leq 510} \gamma_{t} y_{t} \equiv \omega_{(1)} 0
$$

where $\gamma_{t} \in \mathbb{Z} / 2$. From a result in $[62], \operatorname{dim} Q \mathscr{P}_{4}^{+}\left(\omega_{(1)}\right)=26$, with the basis $\left\{\left[u_{j}\right]_{\omega_{(1)}}: 1 \leq j \leq 26\right\}$, where
$u_{1} \cdot x_{1} x_{2} x_{3}^{6} x_{4}^{14}$,
$u_{2} . x_{1} x_{2} x_{3}^{14} x_{4}^{6}$,
$u_{6} \cdot x_{1} x_{2}^{2} x_{3}^{12} x_{4}^{7}$, $u_{7} . x_{1} x_{2}^{2} x_{3}^{13} x_{4}^{6}$,
$u_{11} . x_{1} x_{2}^{3} x_{3}^{12} x_{4}^{6}$,
$u_{12} . x_{1} x_{2}^{3} x_{3}^{14} x_{4}^{4}$,
$u_{3} . x_{1} x_{2}^{2} x_{3}^{4} x_{4}^{15}$, $u_{8} . x_{1} x_{2}^{2} x_{3}^{15} x_{4}^{4}$,
$u_{4} . x_{1} x_{2}^{2} x_{3}^{5} x_{4}^{14}$,
$u_{5} . x_{1} x_{2}^{2} x_{3}^{7} x_{4}^{12}$,
$u_{16} \cdot x_{1} x_{2}^{15} x_{3}^{2} x_{4}^{4}$,
$u_{17} \cdot x_{1}^{3} x_{2} x_{3}^{4} x_{4}^{14}$,
$u_{13} . x_{1} x_{2}^{6} x_{3} x_{4}^{14}$,
$u_{9} . x_{1} x_{2}^{3} x_{3}^{4} x_{4}^{14}$,
$u_{10} . x_{1} x_{2}^{3} x_{3}^{6} x_{4}^{12}$,
$u_{21} . x_{1}^{3} x_{2}^{5} x_{3}^{2} x_{4}^{12}$,
$u_{26} \cdot x_{1}^{15} x_{2} x_{3}^{2} x_{4}^{4}$.
$u_{18} \cdot x_{1}^{3} x_{2} x_{3}^{6} x_{4}^{12}$,
$u_{14} . x_{1} x_{2}^{7} x_{3}^{2} x_{4}^{12}$,
$u_{19} \cdot x_{1}^{3} x_{2} x_{3}^{12} x_{4}^{6}$,
$u_{15} \cdot x_{1} x_{2}^{14} x_{3} x_{4}^{6}$,
$u_{22} . x_{1}^{3} x_{2}^{5} x_{3}^{6} x_{4}^{8}$,
$u_{23} \cdot x_{1}^{3} x_{2}^{5} x_{3}^{10} x_{4}^{4}$,
$u_{20} \cdot x_{1}^{3} x_{2} x_{3}^{14} x_{4}^{4}$,
$u_{25} \cdot x_{1}^{7} x_{2} x_{3}^{2} x_{4}^{12}$, 
Consider the homomorphism $\pi_{(1 ; 2)}: \mathscr{P}_{5} \rightarrow \mathscr{P}_{4}$. By a direct computation using Theorem 3.1.3 and Proposition 3.2.1, we have

$$
\begin{aligned}
\pi_{(1 ; 2)}(\mathcal{S}) & \equiv \omega_{(1)}\left(\gamma_{473}+\gamma_{475}+\gamma_{481}+\gamma_{483}\right) u_{1}+\gamma_{477} u_{6}+\gamma_{478} u_{7} \\
& +\left(\gamma_{476}+\gamma_{477}+\gamma_{478}\right) u_{4}+\gamma_{483}\left(u_{9}+u_{14}\right)+\gamma_{481}\left(u_{10}+u_{11}\right) \\
& +\left(\gamma_{475}+\gamma_{476}+\gamma_{477}+\gamma_{478}\right) u_{13}+\left(\gamma_{470}+\gamma_{481}+\gamma_{484}\right) u_{18} \\
& +\left(\gamma_{471}+\gamma_{478}+\gamma_{481}+\gamma_{484}\right) u_{19}+\gamma_{472} u_{20}+\left(\gamma_{479}+\gamma_{484}\right) u_{21} \\
& +\left(\gamma_{480}+\gamma_{481}+\gamma_{484}\right) u_{22}+\gamma_{482} u_{23}+\gamma_{485} u_{24}+\gamma_{495} u_{25}+\gamma_{497} u_{26} \\
& +\left(\gamma_{474}+\gamma_{478}+\gamma_{481}+\gamma_{484}\right) u_{2}+\gamma_{476} u_{5}+\left(\gamma_{469}+\gamma_{475}\right) u_{17}+\gamma_{484} u_{15} \equiv_{\omega_{(1)}} 0 .
\end{aligned}
$$

This relation implies

$$
\begin{aligned}
\gamma_{469} & =\gamma_{470}=\gamma_{471}=\gamma_{472}=\gamma_{473}=\gamma_{474}=\gamma_{475}=\gamma_{476} \\
& =\gamma_{477}=\gamma_{478}=\gamma_{479}=\gamma_{480}=\gamma_{481}=\gamma_{482}=\gamma_{483} \\
& =\gamma_{484}=\gamma_{485}=\gamma_{495}=\gamma_{497}=0
\end{aligned}
$$

Substituting (3.2) into the relation (3.1), we have

$$
\sum_{461 \leq t \leq 468} \gamma_{t} y_{t}+\sum_{486 \leqslant t \leqslant 494} \gamma_{t} y_{t}+\gamma_{496} y_{36}+\sum_{498 \leq t \leq 510} \gamma_{t} y_{t} \equiv_{\omega_{(1)}} 0 .
$$

Applying the homomorphisms $\pi_{(1 ; 3)}, \pi_{(1 ; 4)}: \mathscr{P}_{5} \rightarrow \mathscr{P}_{4}$ to $(3.3)$, we get

$$
\left\{\begin{array}{l}
\gamma_{t}=0, t \in \mathbb{J}, \\
\gamma_{464}=\gamma_{487}=\gamma_{499}=\gamma_{508}, \gamma_{468}=\gamma_{494}=\gamma_{506}=\gamma_{509}, \gamma_{498}=\gamma_{502}, \\
\gamma_{467}+\gamma_{468}+\gamma_{503}=\gamma_{467}+\gamma_{468}+\gamma_{505}=0 \\
\gamma_{462}+\gamma_{468}+\gamma_{502}+\gamma_{508}=\gamma_{463}+\gamma_{468}+\gamma_{502}+\gamma_{508}=0 \\
\gamma_{466}+\gamma_{501}+\gamma_{502}+\gamma_{508}=\gamma_{466}+\gamma_{468}+\gamma_{504}+\gamma_{507}+\gamma_{508}=0
\end{array}\right.
$$

Here $\mathbb{J}=\{461,465,486,488,489,490,491,492,493,496,500\}$. Then, combining (3.2), (3.4), and the relation $\pi_{(1 ; 5)}(\mathcal{S}) \equiv \omega_{\omega_{(1)}} 0$, we obtain $\gamma_{t}=0$ for $461 \leq t \leq 510$. The proposition is proved.

Using a similar technique as mentioned in the proof of Proposition 3.3.10, we obtain

\section{Proposition 3.3.14.}

(I) $\mathscr{B}_{5}^{+}\left(\omega_{(2)}\right)=\mathscr{B}^{+}\left(5, \omega_{(2)}\right) \cup \mathcal{E}$, where $\mathcal{E}$ is the set of the following monomials:

$\begin{array}{lllll}x_{1} x_{2}^{2} x_{3}^{2} x_{4}^{3} x_{5}^{14}, & x_{1} x_{2}^{2} x_{3}^{3} x_{4}^{2} x_{5}^{14}, & x_{1} x_{2}^{2} x_{3}^{3} x_{4}^{6} x_{5}^{10}, & x_{1} x_{2}^{2} x_{3}^{3} x_{4}^{14} x_{5}^{2}, & x_{1} x_{2}^{3} x_{3}^{2} x_{4}^{2} x_{5}^{14}, \\ x_{1} x_{2}^{3} x_{3}^{2} x_{4}^{6} x_{5}^{10}, & x_{1} x_{2}^{3} x_{3}^{2} x_{4}^{14} x_{5}^{2}, & x_{1} x_{2}^{3} x_{3}^{6} x_{4}^{2} x_{5}^{10}, & x_{1} x_{2}^{3} x_{3}^{6} x_{4}^{10} x_{5}^{2}, & x_{1} x_{2}^{3} x_{3}^{14} x_{4}^{2} x_{5}^{2}, \\ x_{1}^{3} x_{2} x_{3}^{2} x_{4}^{2} x_{5}^{14}, & x_{1}^{3} x_{2} x_{3}^{2} x_{4}^{6} x_{5}^{10}, & x_{1}^{3} x_{2} x_{3}^{2} x_{4}^{14} x_{5}^{2}, & x_{1}^{3} x_{2} x_{3}^{6} x_{4}^{2} x_{5}^{10}, & x_{1}^{3} x_{2} x_{3}^{6} x_{4}^{10} x_{5}^{2}, \\ x_{1}^{3} x_{2} x_{3}^{14} x_{4}^{2} x_{5}^{2}, & x_{1}^{3} x_{2}^{5} x_{3}^{2} x_{4}^{2} x_{5}^{10}, & x_{1}^{3} x_{2}^{5} x_{3}^{2} x_{4}^{10} x_{5}^{2}, & x_{1}^{3} x_{2}^{5} x_{3}^{10} x_{4}^{2} x_{5}^{2}, & x_{1}^{3} x_{2}^{13} x_{3}^{2} x_{4}^{2} x_{5}^{2},\end{array}$

(II) $\mathscr{B}_{5}^{+}\left(\omega_{(3)}\right)=\left\{x_{1} x_{2}^{3} x_{3}^{6} x_{4}^{6} x_{5}^{6}, x_{1}^{3} x_{2} x_{3}^{6} x_{4}^{6} x_{5}^{6}, x_{1}^{3} x_{2}^{5} x_{3}^{2} x_{4}^{6} x_{5}^{6}, x_{1}^{3} x_{2}^{5} x_{3}^{6} x_{4}^{2} x_{5}^{6}, x_{1}^{3} x_{2}^{5} x_{3}^{6} x_{4}^{6} x_{5}^{2}\right\}$,

(III) $\mathscr{B}_{5}^{+}\left(\omega_{(4)}\right)=\bar{\Phi}^{+}\left(\mathscr{B}_{4}\left(\omega_{(4)}\right) \bigcup\left(\bigcup_{1 \leq k \leq 5} \overline{\mathscr{B}}^{+}\left(k, \omega_{(4)}\right)\right)\right.$,

(IV) $\mathscr{B}_{5}^{+}\left(\omega_{(5)}\right)=\bar{\Phi}^{+}\left(\mathscr{B}_{4}\left(\omega_{(5)}\right) \bigcup\left(\bigcup_{1 \leq k \leq 5} \overline{\mathscr{B}}^{+}\left(k, \omega_{(5)}\right)\right)\right.$.

A direct computation shows: $\left|\mathscr{B}_{5}^{+}\left(\omega_{(2)}\right)\right|=25,\left|\mathscr{B}_{5}^{+}\left(\omega_{(4)}\right)\right|=300$ and $\left|\mathscr{B}_{5}^{+}\left(\omega_{(5)}\right)\right|=125$. On the other hand, by Remark 3.3.9, we have $\left(Q \mathscr{P}_{5}\right)_{22}^{+} \cong \bigoplus_{1 \leq j \leq 5} Q \mathscr{P}_{5}^{+}\left(\omega_{(j)}\right)$. Combining this with the above results, we obtain

Corollary 3.3.15. $\left(Q \mathscr{P}_{5}^{+}\right)_{22}$ is the $\mathbb{Z} / 2$-vector space of dimension 505 with a basis consisting of all the classes represented by the monomials $y_{22, t}, 1 \leq t \leq 505$, which are described in Sect.6.5 of the online version [44].

\section{Structure of the kernel of Kameko's map $\left(\widetilde{S q_{*}^{0}}\right)_{(5,47)}$}

The following weight vectors that have the same degrees are 47 :

$$
\begin{gathered}
\bar{\omega}_{(1)}=(3,2,2,2,1), \bar{\omega}_{(2)}=(3,2,4,1,1), \bar{\omega}_{(3)}=(3,2,4,3), \\
\bar{\omega}_{(4)}=(3,4,3,1,1), \bar{\omega}_{(5)}=(3,4,3,3) .
\end{gathered}
$$

From Remarks 3.3.8 and 3.3.9, we conclude that if $X \in \mathscr{B}_{5}(47)$ and $[X]$ belongs to the kernel of $\left(\widetilde{S q_{*}^{0}}\right)_{(5,47)}$ then the weight vector of $X$ is one of the above sequences $\bar{\omega}_{(k)}, 1 \leq k \leq 5$. This implies that the dimension of $\operatorname{Ker}\left(\widetilde{S q_{*}^{0}}\right)_{(5,47)}$ is equal to the sum of the dimensions of $Q \mathscr{P}_{5}^{0}$ and $Q \mathscr{P}_{5}^{+}\left(\bar{\omega}_{(k)}\right)$ in degree 47 for all $1 \leq k \leq 5$. Since $\left(Q \mathscr{P}_{5}^{0}\right)_{47}$ is isomorphic to $\bigoplus_{1 \leq t \leq 4} \bigoplus_{1 \leq \ell \leq\left(\begin{array}{c}5 \\ t\end{array}\right)}\left(Q \mathscr{P}_{t}^{+}\right)_{47}$, by a direct computation using a result in [21], [37] and [62], we claim

$$
\operatorname{dim}\left(Q \mathscr{P}_{5}^{0}\right)_{47}=\left(\begin{array}{l}
5 \\
3
\end{array}\right) .14+\left(\begin{array}{l}
5 \\
4
\end{array}\right) .84=560 .
$$

Furthermore, $\mathscr{B}_{5}^{0}(47)=\mathscr{B}_{5}^{0}\left(\bar{\omega}_{(1)}\right)=\bar{\Phi}^{0}\left(\mathscr{B}_{4}(47)\right)=\left\{y_{47, i}: 1 \leq i \leq 560\right\}$, where the monomials $y_{47, i} \in \mathscr{B}_{5}^{0}(47)$ are explicitly described in Sect.6.6. of the online version [44].

We now determine the $\mathbb{Z} / 2$-subspaces $Q \mathscr{P}_{5}^{+}\left(\bar{\omega}_{(k)}\right)$ for $k=1,2, \ldots, 5$. 
Lemma 3.3.16. The following monomials are strictly inadmissible:

I) $X_{1}=x_{1}^{3} x_{2}^{12} x_{k} x_{\ell}^{3} x_{m}^{12}, X_{2}=x_{1}^{3} x_{2}^{4} x_{k}^{3} x_{\ell}^{8} x_{m}^{13}, X_{3}=x_{1} x_{2}^{14} x_{k}^{2} x_{\ell} x_{m}^{13}$,

$X_{5}=x_{1}^{3} x_{2}^{14} x_{k}^{12} x_{\ell} x_{m}, X_{6}=x_{1}^{7} x_{2}^{10} x_{k}^{12} x_{\ell} x_{m}, X_{7}=x_{1}^{3} x_{2}^{2} x_{k}^{12} x_{\ell} x_{m}^{13}$,

$X_{8}=x_{1}^{3} x_{2}^{12} x_{k}^{2} x_{\ell} x_{m}^{13}, X_{9}=x_{1}^{15} x_{2}^{2} x_{k} x_{\ell}^{4} x_{m}^{9}, X_{10}=x_{1}^{15} x_{2}^{2} x_{k} x_{\ell}^{5} x_{m}^{8}$,

$X_{11}=x_{1}^{7} x_{2}^{2} x_{k} x_{\ell}^{8} x_{m}^{13}, X_{12}=x_{1}^{7} x_{2}^{2} x_{k} x_{\ell}^{9} x_{m}^{12}, X_{13}=x_{1}^{15} x_{2}^{2} x_{k}^{12} x_{\ell} x_{m}$,

$X_{14}=x_{1}^{3} x_{2}^{4} x_{k} x_{\ell}^{8} x_{m}^{15}, X_{15}=x_{1}^{3} x_{2}^{4} x_{k} x_{\ell}^{9} x_{m}^{14}, X_{16}=x_{1}^{3} x_{2}^{14} x_{k} x_{\ell}^{4} x_{m}^{9}$,

$X_{17}=x_{1}^{3} x_{2}^{5} x_{k} x_{\ell}^{8} x_{m}^{14}, X_{18}=x_{1}^{3} x_{2}^{6} x_{k} x_{\ell}^{8} x_{m}^{13}, X_{19}=x_{1}^{3} x_{2}^{6} x_{k} x_{\ell}^{9} x_{m}^{12}$,

$X_{20}=x_{1}^{7} x_{2}^{10} x_{k} x_{\ell}^{4} x_{m}^{9}, X_{21}=x_{1}^{7} x_{2}^{10} x_{k} x_{\ell}^{5} x_{m}^{8}, X_{22}=x_{1}^{3} x_{2}^{2} x_{k}^{5} x_{\ell}^{8} x_{m}^{13}$,

$X_{23}=x_{1}^{3} x_{2}^{2} x_{k}^{5} x_{\ell}^{9} x_{m}^{12}, X_{24}=x_{1}^{7} x_{2}^{2} x_{k}^{5} x_{\ell}^{8} x_{m}^{9}, X_{25}=x_{1} x_{2}^{2} x_{k}^{14} x_{\ell} x_{m}^{13}$,

$X_{26}=x_{1}^{3} x_{2}^{6} x_{k}^{5} x_{\ell}^{8} x_{m}^{9}, X_{27}=x_{1}^{3} x_{2}^{12} x_{k}^{14} x_{\ell} x_{m}$. Here $(k, \ell, m)$ is a permutation of $(3,4,5)$;

II) $X_{27}=x_{i}^{3} x_{j}^{2} x_{k}^{13} x_{\ell}^{4} x_{m}^{9}, j<k, X_{28}=x_{i} x_{j}^{2} x_{k}^{6} x_{\ell}^{9} x_{m}^{13}, X_{29}=x_{i}^{3} x_{j}^{4} x_{k}^{6} x_{\ell}^{9} x_{m}^{9}$, where $(i, j, k, \ell, m)$ is a permutation of $(1,2,3,4,5)$.

Proof. It is easy to see that $\omega\left(X_{t}\right)=\omega^{*}:=(3,2,2,2)$ for $1 \leq t \leq 29$. We prove the lemma for the monomials $X_{1}=x_{1}^{3} x_{2}^{12} x_{k} x_{\ell}^{3} x_{m}^{12}$ and $X_{2}=x_{1}^{3} x_{2}^{4} x_{k}^{3} x_{\ell}^{8} x_{m}^{13}$, where $(k, \ell, m)$ is a permutation of $(3,4,5)$. The others can be proved by a similar technique. We have

$$
\begin{aligned}
X_{1}= & x_{1}^{2} x_{2}^{11} x_{k} x_{\ell}^{5} x_{m}^{12}+x_{1}^{2} x_{2}^{11} x_{k}^{4} x_{\ell}^{5} x_{m}^{9}+x_{1}^{2} x_{2}^{11} x_{k}^{8} x_{\ell}^{5} x_{m}^{5}+x_{1}^{2} x_{2}^{13} x_{k} x_{\ell}^{3} x_{m}^{12} \\
& +x_{1}^{2} x_{2}^{13} x_{k}^{4} x_{\ell}^{3} x_{m}^{9}+x_{1}^{2} x_{2}^{13} x_{k}^{8} x_{\ell}^{3} x_{m}^{5}+x_{1}^{3} x_{2}^{3} x_{k}^{8} x_{\ell}^{5} x_{m}^{12}+x_{1}^{3} x_{2}^{5} x_{k}^{8} x_{\ell}^{5} x_{m}^{10} \\
& +x_{1}^{3} x_{2}^{5} x_{k}^{8} x_{\ell}^{6} x_{m}^{9}+x_{1}^{3} x_{2}^{7} x_{k}^{4} x_{\ell}^{8} x_{m}^{9}+x_{1}^{3} x_{2}^{7} x_{k}^{8} x_{\ell}^{5} x_{m}^{8}+x_{1}^{3} x_{2}^{7} x_{k}^{8} x_{\ell}^{8} x_{m}^{5} \\
& +x_{1}^{3} x_{2}^{9} x_{k}^{2} x_{\ell}^{5} x_{m}^{12}+x_{1}^{3} x_{2}^{9} x_{k}^{4} x_{\ell}^{3} x_{m}^{12}+x_{1}^{3} x_{2}^{9} x_{k}^{4} x_{\ell}^{5} x_{m}^{10}+x_{1}^{3} x_{2}^{9} x_{k}^{4} x_{\ell}^{6} x_{m}^{9} \\
& +x_{1}^{3} x_{2}^{9} x_{k}^{8} x_{\ell}^{5} x_{m}^{6}+x_{1}^{3} x_{2}^{9} x_{k}^{8} x_{\ell}^{6} x_{m}^{5}+x_{1}^{3} x_{2}^{11} x_{k} x_{\ell}^{4} x_{m}^{12} \\
& +S q^{1}\left(g_{1}\right)+S q^{2}\left(g_{2}\right)+S q^{4}\left(g_{4}\right)+S q^{8}\left(x_{1}^{6} x_{2}^{5} x_{k}^{4} x_{\ell}^{3} x_{m}^{5}\right) \text { modulo}\left(\mathscr{P}_{5}^{-}\left(\omega^{*}\right)\right), \text { where } \\
g_{1}= & x_{1}^{3} x_{2}^{7} x_{k} x_{\ell}^{3} x_{m}^{16}+x_{1}^{3} x_{2}^{11} x_{k} x_{\ell}^{3} x_{m}^{12}+x_{1}^{5} x_{2}^{3} x_{k}^{8} x_{\ell}^{5} x_{m}^{9}+x_{1}^{5} x_{2}^{7} x_{k}^{4} x_{\ell}^{5} x_{m}^{9}+x_{1}^{5} x_{2}^{7} x_{k}^{8} x_{\ell}^{5} x_{m}^{5}, \\
g_{2}, & x_{1}^{2} x_{2}^{11} x_{k} x_{\ell}^{3} x_{m}^{12}+x_{1}^{2} x_{2}^{11} x_{k}^{4} x_{\ell}^{3} x_{m}^{9}+x_{1}^{2} x_{2}^{11} x_{k}^{8} x_{\ell}^{3} x_{m}^{5}+x_{1}^{3} x_{2}^{3} x_{k}^{8} x_{\ell}^{5} x_{m}^{10}+x_{1}^{3} x_{2}^{3} x_{k}^{8} x_{\ell}^{6} x_{m}^{9} \\
& +x_{1}^{3} x_{2}^{7} x_{k}^{4} x_{\ell}^{5} x_{m}^{12}+x_{1}^{3} x_{2}^{7} x_{k}^{4} x_{\ell}^{6} x_{m}^{9}+x_{1}^{3} x_{2}^{7} x_{k}^{8} x_{\ell}^{5} x_{m}^{6}+x_{1}^{3} x_{2}^{7} x_{k}^{8} x_{\ell}^{6} x_{m}^{5}+x_{1}^{5} x_{2}^{7} x_{k}^{2} x_{\ell}^{3} x_{m}^{12} \\
& +x_{1}^{6} x_{2}^{3} x_{k}^{8} x_{\ell}^{3} x_{m}^{9}+x_{1}^{6} x_{2}^{7} x_{k}^{4} x_{\ell}^{3} x_{m}^{9}+x_{1}^{6} x_{2}^{7} x_{k}^{8} x_{\ell}^{3} x_{m}^{5}, \\
= & x_{1}^{3} x_{2}^{7} x_{k}^{2} x_{\ell}^{3} x_{m}^{12}+x_{1}^{4} x_{2}^{7} x_{k}^{4} x_{\ell}^{3} x_{m}^{9}+x_{1}^{4} x_{2}^{7} x_{k}^{8} x_{\ell}^{3} x_{m}^{5}+x_{1}^{10} x_{2}^{5} x_{k}^{4} x_{\ell}^{3} x_{m}^{5} .
\end{aligned}
$$

This equality implies that $X_{1}$ is strictly inadmissible.

Next, we show that $X_{2}$ is also strictly inadmissible. Indeed, using Cartan's formula, we obtain

$$
\begin{aligned}
& X_{2}=x_{1}^{2} x_{2} x_{k}^{3} x_{\ell}^{12} x_{m}^{13}+x_{1}^{2} x_{2} x_{k}^{5} x_{\ell}^{9} x_{m}^{14}+x_{1}^{2} x_{2} x_{k}^{5} x_{\ell}^{10} x_{m}^{13}+x_{1}^{2} x_{2} x_{k}^{6} x_{\ell}^{9} x_{m}^{13}+x_{1}^{2} x_{2} x_{k}^{10} x_{\ell}^{5} x_{m}^{13} \\
& +x_{1}^{2} x_{2} x_{k}^{12} x_{\ell}^{5} x_{m}^{11}+x_{1}^{2} x_{2}^{5} x_{k} x_{\ell}^{9} x_{m}^{14}+x_{1}^{2} x_{2}^{5} x_{k} x_{\ell}^{10} x_{m}^{13}+x_{1}^{2} x_{2}^{5} x_{k}^{8} x_{\ell}^{3} x_{m}^{13}+x_{1}^{2} x_{2}^{5} x_{k}^{8} x_{\ell}^{5} x_{m}^{11} \\
& +x_{1}^{3} x_{2} x_{k}^{4} x_{\ell}^{9} x_{m}^{14}+x_{1}^{3} x_{2} x_{k}^{4} x_{\ell}^{10} x_{m}^{13}+x_{1}^{3} x_{2} x_{k}^{6} x_{\ell}^{8} x_{m}^{13}+x_{1}^{3} x_{2} x_{k}^{8} x_{\ell}^{6} x_{m}^{13}+x_{1}^{3} x_{2}^{2} x_{k}^{4} x_{\ell}^{9} x_{m}^{13} \\
& +x_{1}^{3} x_{2}^{2} x_{k}^{5} x_{\ell}^{8} x_{m}^{13}+x_{1}^{3} x_{2}^{3} x_{k}^{8} x_{\ell}^{4} x_{m}^{13}+x_{1}^{3} x_{2}^{3} x_{k}^{8} x_{\ell}^{5} x_{m}^{12}+x_{1}^{3} x_{2}^{4} x_{k} x_{\ell}^{9} x_{m}^{14}+x_{1}^{3} x_{2}^{4} x_{k} x_{\ell}^{10} x_{m}^{13} \\
& +x_{1}^{3} x_{2}^{4} x_{k}^{2} x_{\ell}^{9} x_{m}^{13}+S q^{1}\left(Z_{1}\right)+S q^{2}\left(Z_{2}\right)+S q^{4}\left(Z_{4}\right)+S q^{8}\left(x_{1}^{3} x_{2}^{4} x_{k}^{4} x_{\ell}^{5} x_{m}^{7}\right) \text { modulo }\left(\mathscr{P}_{5}^{-}\left(\omega^{*}\right)\right) \text {, where } \\
& Z_{1}=x_{1}^{3} x_{2} x_{k}^{3} x_{\ell}^{5} x_{m}^{18}+x_{1}^{3} x_{2} x_{k}^{3} x_{\ell}^{6} x_{m}^{17}+x_{1}^{3} x_{2} x_{k}^{3} x_{\ell}^{9} x_{m}^{14}+x_{1}^{3} x_{2} x_{k}^{3} x_{\ell}^{10} x_{m}^{13} \\
& +x_{1}^{3} x_{2} x_{k}^{6} x_{\ell}^{9} x_{m}^{11}+x_{1}^{3} x_{2} x_{k}^{10} x_{\ell}^{5} x_{m}^{11}+x_{1}^{3} x_{2}^{3} x_{k} x_{\ell}^{6} x_{m}^{17}+x_{1}^{3} x_{2}^{3} x_{k} x_{\ell}^{10} x_{m}^{13} \\
& +x_{1}^{3} x_{2}^{3} x_{k} x_{\ell}^{12} x_{m}^{11}+x_{1}^{3} x_{2}^{3} x_{k} x_{\ell}^{16} x_{m}^{7}+x_{1}^{3} x_{2}^{3} x_{k}^{4} x_{\ell}^{3} x_{m}^{17}+x_{1}^{3} x_{2}^{3} x_{k}^{8} x_{\ell}^{3} x_{m}^{13} \\
& +x_{1}^{3} x_{2}^{3} x_{k}^{8} x_{\ell}^{5} x_{m}^{11}+x_{1}^{3} x_{2}^{3} x_{k}^{8} x_{\ell}^{9} x_{m}^{7}+x_{1}^{3} x_{2}^{4} x_{k}^{5} x_{\ell}^{5} x_{m}^{13}+x_{1}^{5} x_{2}^{5} x_{k} x_{\ell}^{5} x_{m}^{14}, \\
& Z_{2}=x_{1}^{2} x_{2} x_{k}^{3} x_{\ell}^{9} x_{m}^{14}+x_{1}^{2} x_{2} x_{k}^{3} x_{\ell}^{10} x_{m}^{13}+x_{1}^{2} x_{2} x_{k}^{6} x_{\ell}^{9} x_{m}^{11}+x_{1}^{2} x_{2} x_{k}^{10} x_{\ell}^{5} x_{m}^{11} \\
& +x_{1}^{2} x_{2}^{3} x_{k} x_{\ell}^{9} x_{m}^{14}+x_{1}^{2} x_{2}^{3} x_{k} x_{\ell}^{10} x_{m}^{13}+x_{1}^{2} x_{2}^{3} x_{k} x_{\ell}^{12} x_{m}^{11}+x_{1}^{2} x_{2}^{3} x_{k}^{8} x_{\ell}^{3} x_{m}^{13} \\
& +x_{1}^{2} x_{2}^{3} x_{k}^{8} x_{\ell}^{5} x_{m}^{11}+x_{1}^{2} x_{2}^{3} x_{k}^{8} x_{\ell}^{9} x_{m}^{7}+x_{1}^{3} x_{2}^{5} x_{k} x_{\ell}^{6} x_{m}^{14}+x_{1}^{3} x_{2}^{5} x_{k}^{2} x_{\ell}^{5} x_{m}^{14} \\
& +x_{1}^{3} x_{2}^{6} x_{k} x_{\ell}^{5} x_{m}^{14}+x_{1}^{5} x_{2} x_{k}^{6} x_{\ell}^{6} x_{m}^{11}+x_{1}^{5} x_{2}^{2} x_{k}^{2} x_{\ell}^{9} x_{m}^{11}+x_{1}^{5} x_{2}^{2} x_{k}^{3} x_{\ell}^{5} x_{m}^{14} \\
& +x_{1}^{5} x_{2}^{2} x_{k}^{3} x_{\ell}^{6} x_{m}^{13}+x_{1}^{5} x_{2}^{2} x_{k}^{6} x_{\ell}^{5} x_{m}^{11}+x_{1}^{5} x_{2}^{2} x_{k}^{8} x_{\ell}^{3} x_{m}^{11}+x_{1}^{5} x_{2}^{3} x_{k} x_{\ell}^{6} x_{m}^{14} \\
& +x_{1}^{5} x_{2}^{3} x_{k}^{2} x_{\ell}^{6} x_{m}^{13}+x_{1}^{5} x_{2}^{3} x_{k}^{2} x_{\ell}^{8} x_{m}^{11}+x_{1}^{5} x_{2}^{3} x_{k}^{2} x_{\ell}^{10} x_{m}^{9}+x_{1}^{5} x_{2}^{3} x_{k}^{2} x_{\ell}^{12} x_{m}^{7} \\
& +x_{1}^{5} x_{2}^{3} x_{k}^{4} x_{\ell}^{3} x_{m}^{14}+x_{1}^{5} x_{2}^{3} x_{k}^{4} x_{\ell}^{6} x_{m}^{11}+x_{1}^{5} x_{2}^{3} x_{k}^{4} x_{\ell}^{10} x_{m}^{7}+x_{1}^{6} x_{2}^{3} x_{k} x_{\ell}^{5} x_{m}^{14}, \\
& Z_{4}=x_{1}^{3} x_{2} x_{k}^{6} x_{\ell}^{5} x_{m}^{12}+x_{1}^{3} x_{2} x_{k}^{6} x_{\ell}^{6} x_{m}^{11}+x_{1}^{3} x_{2}^{2} x_{k}^{2} x_{\ell}^{9} x_{m}^{11}+x_{1}^{3} x_{2}^{2} x_{k}^{3} x_{\ell}^{6} x_{m}^{13}+x_{1}^{3} x_{2}^{2} x_{k}^{6} x_{\ell}^{5} x_{m}^{11} \\
& +x_{1}^{3} x_{2}^{2} x_{k}^{8} x_{\ell}^{3} x_{m}^{11}+x_{1}^{3} x_{2}^{3} x_{k} x_{\ell}^{6} x_{m}^{14}+x_{1}^{3} x_{2}^{3} x_{k}^{2} x_{\ell}^{6} x_{m}^{13}+x_{1}^{3} x_{2}^{3} x_{k}^{2} x_{\ell}^{8} x_{m}^{11}+x_{1}^{3} x_{2}^{3} x_{k}^{2} x_{\ell}^{10} x_{m}^{9} \\
& +x_{1}^{3} x_{2}^{3} x_{k}^{2} x_{\ell}^{12} x_{m}^{7}+x_{1}^{3} x_{2}^{3} x_{k}^{4} x_{\ell}^{3} x_{m}^{14}+x_{1}^{3} x_{2}^{3} x_{k}^{4} x_{\ell}^{6} x_{m}^{11}+x_{1}^{3} x_{2}^{3} x_{k}^{4} x_{\ell}^{10} x_{m}^{7}+x_{1}^{3} x_{2}^{4} x_{k} x_{\ell}^{5} x_{m}^{14} \\
& +x_{1}^{3} x_{2}^{8} x_{k}^{4} x_{\ell}^{5} x_{m}^{7}+x_{1}^{4} x_{2}^{3} x_{k} x_{\ell}^{5} x_{m}^{14} .
\end{aligned}
$$

The above relations imply that $X_{2}$ is strictly inadmissible. The lemma follows. 
Lemma 3.3.17. The following monomials are strictly inadmissible:

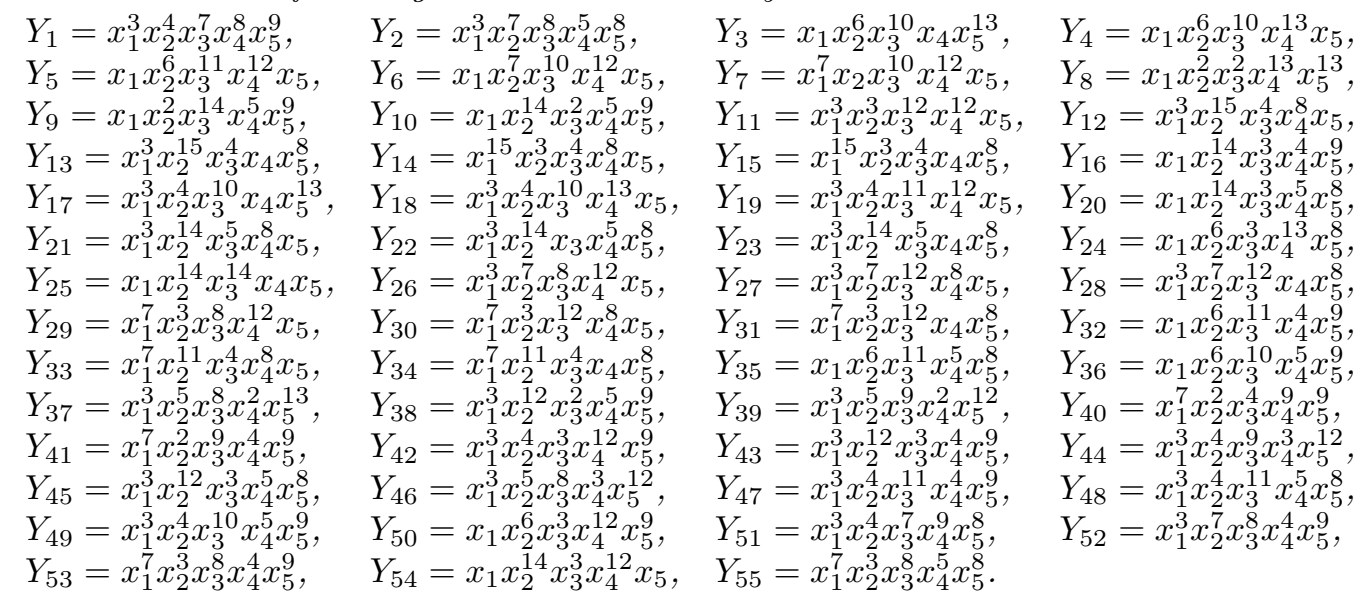

Proof. Note that $\omega\left(Y_{j}\right)=\omega^{*}, j=1,2, \ldots, 55$. We prove this lemma for the monomials $Y_{1}=$ $x_{1}^{3} x_{2}^{4} x_{3}^{7} x_{4}^{8} x_{5}^{9}$, and $Y_{2}=x_{1}^{3} x_{2}^{7} x_{3}^{8} x_{4}^{5} x_{5}^{8}$. The others can be proved by a similar computation. A direct computation shows:

$$
\begin{aligned}
& Y_{1}=x_{1}^{2} x_{2} x_{3}^{7} x_{4}^{8} x_{5}^{13}+x_{1}^{2} x_{2} x_{3}^{13} x_{4} x_{5}^{14}+x_{1}^{2} x_{2} x_{3}^{13} x_{4}^{8} x_{5}^{7}+x_{1}^{2} x_{2}^{3} x_{3}^{9} x_{4}^{4} x_{5}^{13}+x_{1}^{2} x_{2}^{3} x_{3}^{12} x_{4} x_{5}^{13} \\
& +x_{1}^{2} x_{2}^{5} x_{3}^{9} x_{4}^{4} x_{5}^{11}+x_{1}^{2} x_{2}^{5} x_{3}^{9} x_{4}^{8} x_{5}^{7}+x_{1}^{2} x_{2}^{5} x_{3}^{12} x_{4} x_{5}^{11}+x_{1}^{3} x_{2} x_{3}^{5} x_{4}^{8} x_{5}^{14}+x_{1}^{3} x_{2} x_{3}^{7} x_{4}^{8} x_{5}^{12} \\
& +x_{1}^{3} x_{2} x_{3}^{12} x_{4} x_{5}^{14}+x_{1}^{3} x_{2} x_{3}^{12} x_{4}^{4} x_{5}^{11}+x_{1}^{3} x_{2}^{2} x_{3}^{9} x_{4}^{4} x_{5}^{13}+x_{1}^{3} x_{2}^{3} x_{3}^{9} x_{4}^{4} x_{5}^{12}+x_{1}^{3} x_{2}^{3} x_{3}^{12} x_{4} x_{5}^{12} \\
& +x_{1}^{3} x_{2}^{3} x_{3}^{12} x_{4}^{4} x_{5}^{9}+S q^{1}\left(u_{1}\right)+S q^{2}\left(u_{2}\right)+S q^{4}\left(u_{4}\right)+S q^{8}\left(u_{8}\right) \text { modulo }\left(\mathscr{P}_{5}^{-}\left(\omega^{*}\right)\right) \text {, where } \\
& u_{1}=x_{1}^{3} x_{2} x_{3}^{5} x_{4}^{8} x_{5}^{13}+x_{1}^{3} x_{2} x_{3}^{7} x_{4} x_{5}^{18}+x_{1}^{3} x_{2} x_{3}^{9} x_{4}^{4} x_{5}^{13}+x_{1}^{3} x_{2} x_{3}^{11} x_{4} x_{5}^{14} \\
& +x_{1}^{3} x_{2}^{3} x_{3}^{9} x_{4}^{4} x_{5}^{11}+x_{1}^{3} x_{2}^{3} x_{3}^{9} x_{4}^{8} x_{5}^{7}+x_{1}^{3} x_{2}^{3} x_{3}^{12} x_{4} x_{5}^{11}+x_{1}^{3} x_{2}^{3} x_{3}^{16} x_{4} x_{5}^{7} \\
& +x_{1}^{3} x_{2}^{4} x_{3}^{9} x_{4} x_{5}^{13}+x_{1}^{5} x_{2} x_{3}^{5} x_{4}^{8} x_{5}^{11}+x_{1}^{5} x_{2} x_{3}^{7} x_{4}^{8} x_{5}^{9} \text {, } \\
& u_{2}=x_{1}^{2} x_{2} x_{3}^{7} x_{4}^{8} x_{5}^{11}+x_{1}^{2} x_{2} x_{3}^{11} x_{4} x_{5}^{14}+x_{1}^{2} x_{2} x_{3}^{11} x_{4}^{8} x_{5}^{7}+x_{1}^{2} x_{2}^{3} x_{3}^{9} x_{4}^{4} x_{5}^{11} \\
& +x_{1}^{2} x_{2}^{3} x_{3}^{9} x_{4}^{8} x_{5}^{7}+x_{1}^{2} x_{2}^{3} x_{3}^{12} x_{4} x_{5}^{11}+x_{1}^{3} x_{2} x_{3}^{6} x_{4}^{8} x_{5}^{11}+x_{1}^{3} x_{2} x_{3}^{7} x_{4}^{8} x_{5}^{10} \\
& +x_{1}^{3} x_{2} x_{3}^{10} x_{4}^{4} x_{5}^{11}+x_{1}^{3} x_{2}^{2} x_{3}^{5} x_{4}^{8} x_{5}^{11}+x_{1}^{3} x_{2}^{2} x_{3}^{7} x_{4}^{8} x_{5}^{9}+x_{1}^{3} x_{2}^{2} x_{3}^{9} x_{4}^{4} x_{5}^{11} \\
& +x_{1}^{5} x_{2} x_{3}^{7} x_{4}^{2} x_{5}^{14}+x_{1}^{5} x_{2}^{2} x_{3}^{7} x_{4} x_{5}^{14}+x_{1}^{5} x_{2}^{2} x_{3}^{9} x_{4}^{2} x_{5}^{11}+x_{1}^{5} x_{2}^{3} x_{3}^{10} x_{4}^{2} x_{5}^{9} \\
& +x_{1}^{5} x_{2}^{3} x_{3}^{10} x_{4}^{4} x_{5}^{7}+x_{1}^{5} x_{2}^{3} x_{3}^{12} x_{4}^{2} x_{5}^{7}+x_{1}^{6} x_{2} x_{3}^{7} x_{4}^{8} x_{5}^{7}, \\
& u_{4}=x_{1}^{3} x_{2} x_{3}^{7} x_{4}^{2} x_{5}^{14}+x_{1}^{3} x_{2}^{2} x_{3}^{7} x_{4} x_{5}^{14}+x_{1}^{3} x_{2}^{2} x_{3}^{9} x_{4}^{2} x_{5}^{11}+x_{1}^{3} x_{2}^{2} x_{3}^{10} x_{4} x_{5}^{11} \\
& +x_{1}^{3} x_{2}^{3} x_{3}^{10} x_{4}^{2} x_{5}^{9}+x_{1}^{3} x_{2}^{3} x_{3}^{10} x_{4}^{4} x_{5}^{7}+x_{1}^{3} x_{2}^{3} x_{3}^{12} x_{4}^{2} x_{5}^{7}+x_{1}^{3} x_{2}^{8} x_{3}^{5} x_{4}^{4} x_{5}^{7} \\
& +x_{1}^{4} x_{2} x_{3}^{7} x_{4}^{8} x_{5}^{7}+x_{1}^{10} x_{2} x_{3}^{5} x_{4}^{4} x_{5}^{7} \text {, } \\
& u_{8}=x_{1}^{3} x_{2}^{4} x_{3}^{5} x_{4}^{4} x_{5}^{7}+x_{1}^{6} x_{2} x_{3}^{5} x_{4}^{4} x_{5}^{7} \text {. }
\end{aligned}
$$

By a similar computation, we have

$$
\begin{aligned}
& Y_{2}=x_{1}^{2} x_{2}^{7} x_{3}^{8} x_{4}^{5} x_{5}^{9}+x_{1}^{2} x_{2}^{11} x_{3} x_{4}^{5} x_{5}^{12}+x_{1}^{2} x_{2}^{11} x_{3}^{8} x_{4}^{5} x_{5}^{5}+x_{1}^{2} x_{2}^{13} x_{3} x_{4}^{5} x_{5}^{10}+x_{1}^{2} x_{2}^{13} x_{3} x_{4}^{9} x_{5}^{6} \\
& +x_{1}^{2} x_{2}^{13} x_{3}^{8} x_{4}^{3} x_{5}^{5}+x_{1}^{3} x_{2}^{3} x_{3} x_{4}^{12} x_{5}^{12}+x_{1}^{3} x_{2}^{3} x_{3}^{4} x_{4}^{9} x_{5}^{12}+x_{1}^{3} x_{2}^{3} x_{3}^{8} x_{4}^{5} x_{5}^{12}+x_{1}^{3} x_{2}^{5} x_{3}^{4} x_{4}^{9} x_{5}^{10} \\
& +x_{1}^{3} x_{2}^{5} x_{3}^{4} x_{4}^{10} x_{5}^{9}+x_{1}^{3} x_{2}^{5} x_{3}^{8} x_{4}^{5} x_{5}^{10}+x_{1}^{3} x_{2}^{5} x_{3}^{8} x_{4}^{6} x_{5}^{9}+x_{1}^{3} x_{2}^{5} x_{3}^{8} x_{4}^{9} x_{5}^{6}+x_{1}^{3} x_{2}^{5} x_{3}^{8} x_{4}^{10} x_{5}^{5} \\
& +x_{1}^{3} x_{2}^{7} x_{3} x_{4}^{8} x_{5}^{12}+x_{1}^{3} x_{2}^{7} x_{3} x_{4}^{12} x_{5}^{8}+x_{1}^{3} x_{2}^{7} x_{3}^{4} x_{4}^{9} x_{5}^{8}+x_{1}^{3} x_{2}^{7} x_{3}^{8} x_{4}^{4} x_{5}^{9} \\
& +S q^{1}\left(v_{1}\right)+S q^{2}\left(v_{2}\right)+S q^{4}\left(v_{4}\right)+S q^{8}\left(v_{8}\right) \text { modulo }\left(\mathscr{P}_{5}^{-}\left(\omega^{*}\right)\right) \text {, where } \\
& v_{1}=x_{1}^{5} x_{2}^{3} x_{3} x_{4}^{9} x_{5}^{12}+x_{1}^{5} x_{2}^{3} x_{3}^{8} x_{4}^{5} x_{5}^{9}+x_{1}^{5} x_{2}^{7} x_{3} x_{4}^{5} x_{5}^{12} \\
& +x_{1}^{5} x_{2}^{7} x_{3} x_{4}^{9} x_{5}^{8}+x_{1}^{5} x_{2}^{7} x_{3}^{4} x_{4}^{5} x_{5}^{9}+x_{1}^{5} x_{2}^{7} x_{3}^{8} x_{4}^{5} x_{5}^{5}, \\
& v_{2}=x_{1}^{2} x_{2}^{7} x_{3}^{8} x_{4}^{3} x_{5}^{9}+x_{1}^{2} x_{2}^{11} x_{3} x_{4}^{5} x_{5}^{10}+x_{1}^{2} x_{2}^{11} x_{3} x_{4}^{9} x_{5}^{6}+x_{1}^{2} x_{2}^{11} x_{3}^{8} x_{4}^{3} x_{5}^{5}+x_{1}^{3} x_{2}^{3} x_{3} x_{4}^{10} x_{5}^{12} \\
& +x_{1}^{3} x_{2}^{3} x_{3}^{2} x_{4}^{9} x_{5}^{12}+x_{1}^{3} x_{2}^{3} x_{3}^{8} x_{4}^{5} x_{5}^{10}+x_{1}^{3} x_{2}^{3} x_{3}^{8} x_{4}^{6} x_{5}^{9}+x_{1}^{3} x_{2}^{7} x_{3} x_{4}^{6} x_{5}^{12}+x_{1}^{3} x_{2}^{7} x_{3} x_{4}^{10} x_{5}^{8} \\
& +x_{1}^{3} x_{2}^{7} x_{3}^{2} x_{4}^{5} x_{5}^{12}+x_{1}^{3} x_{2}^{7} x_{3}^{2} x_{4}^{9} x_{5}^{8}+x_{1}^{3} x_{2}^{7} x_{3}^{4} x_{4}^{5} x_{5}^{10}+x_{1}^{3} x_{2}^{7} x_{3}^{4} x_{4}^{6} x_{5}^{9}+x_{1}^{3} x_{2}^{7} x_{3}^{8} x_{4}^{5} x_{5}^{6} \\
& +x_{1}^{3} x_{2}^{7} x_{3}^{8} x_{4}^{6} x_{5}^{5}+x_{1}^{6} x_{2}^{3} x_{3} x_{4}^{9} x_{5}^{10}+x_{1}^{6} x_{2}^{3} x_{3}^{8} x_{4}^{3} x_{5}^{9}+x_{1}^{6} x_{2}^{7} x_{3} x_{4}^{5} x_{5}^{10}+x_{1}^{6} x_{2}^{7} x_{3} x_{4}^{9} x_{5}^{6} \\
& +x_{1}^{6} x_{2}^{7} x_{3}^{4} x_{4}^{3} x_{5}^{9}+x_{1}^{6} x_{2}^{7} x_{3}^{8} x_{4}^{3} x_{5}^{5}, \\
& v_{4}=x_{1}^{3} x_{2}^{5} x_{3} x_{4}^{6} x_{5}^{12}+x_{1}^{3} x_{2}^{5} x_{3}^{2} x_{4}^{5} x_{5}^{12}+x_{1}^{3} x_{2}^{5} x_{3}^{4} x_{4}^{5} x_{5}^{10}+x_{1}^{3} x_{2}^{5} x_{3}^{4} x_{4}^{6} x_{5}^{9}+x_{1}^{3} x_{2}^{5} x_{3}^{8} x_{4}^{5} x_{5}^{6} \\
& +x_{1}^{3} x_{2}^{5} x_{3}^{8} x_{4}^{6} x_{5}^{5}+x_{1}^{3} x_{2}^{11} x_{3}^{4} x_{4}^{4} x_{5}^{5}+x_{1}^{4} x_{2}^{7} x_{3} x_{4}^{5} x_{5}^{10}+x_{1}^{4} x_{2}^{7} x_{3} x_{4}^{9} x_{5}^{6}+x_{1}^{4} x_{2}^{7} x_{3}^{8} x_{4}^{3} x_{5}^{5} \\
& +x_{1}^{10} x_{2}^{5} x_{3} x_{4}^{5} x_{5}^{6}+x_{1}^{10} x_{2}^{5} x_{3}^{4} x_{4}^{3} x_{5}^{5} \text {, } \\
& v_{8}=x_{1}^{3} x_{2}^{7} x_{3}^{4} x_{4}^{4} x_{5}^{5}+x_{1}^{6} x_{2}^{5} x_{3}^{1} x_{4}^{5} x_{5}^{6}+x_{1}^{6} x_{2}^{5} x_{3}^{4} x_{4}^{3} x_{5}^{5} \text {. }
\end{aligned}
$$

From the above equalities, we see that $Y_{1}$ and $Y_{2}$ are strictly inadmissible. The lemma is proved.

For $s, k \in \mathbb{N}$ and $1 \leq k \leq 5$, we denote

$$
\overline{\mathscr{B}}(k, 47):=\left\{x_{k}^{2^{s}-1} \rho_{(k, 5)}(x) \in\left(\mathscr{P}_{5}\right)_{47}: x \in \mathscr{B}_{4}\left(45-2^{s}\right), \alpha\left(52-2^{s}\right) \leq 4\right\} .
$$

It has been shown (see [28]) that $\overline{\mathscr{B}}(k, 47) \subseteq \mathscr{B}_{5}(47)$ for $k=1,2, \ldots, 5$. We set $\overline{\mathscr{B}}\left(k, \bar{\omega}_{(1)}\right):=$ $\overline{\mathscr{B}}(k, 47) \cap \mathscr{P}_{5}\left(\bar{\omega}_{(1)}\right)$ and $\overline{\mathscr{B}}^{+}\left(k, \bar{\omega}_{(1)}\right):=\overline{\mathscr{B}}\left(k, \bar{\omega}_{(1)}\right) \cap\left(\mathscr{P}_{5}^{+}\right)_{47}$. Then, we obtain the following result. 
Proposition 3.3.18. We have

$$
\begin{aligned}
Q \mathscr{P}_{5}^{+}\left(\bar{\omega}_{(1)}\right) & =\left\langle\left[\bar{\Phi}^{+}\left(\mathscr{B}_{4}\left(\bar{\omega}_{(1)}\right)\right) \bigcup\left(\bigcup_{1 \leq k<5} \overline{\mathscr{B}}^{+}\left(k, \bar{\omega}_{(1)}\right)\right) \bigcup \mathcal{F}\right]_{\bar{\omega}_{(1)}}\right\rangle \\
& =\left\langle\left[y_{47, j}\right]_{\bar{\omega}_{(1)}}: 1 \leq j \leq 370\right\rangle,
\end{aligned}
$$

where the monomials $y_{47, j}, j=1,2, \ldots, 370$, are determined in Sect.6.7 of the online version [44].

The proof of the proposition is based on Lemmas 3.3.16, 3.3.17 and some below results. The follwing lemma is an immediate consequence of a result in Sum [62].

Lemma 3.3.19. The following monomials are strictly inadmissible:

I) $x_{i}^{15} x_{j}^{2} x_{k} x_{\ell}^{13}, x_{i}^{3} x_{j}^{12} x_{k} x_{\ell}^{15}, x_{i}^{3} x_{j}^{14} x_{k} x_{\ell}^{13}$, $x_{i}^{7} x_{j}^{10} x_{k} x_{\ell}^{13}, x_{i}^{3} x_{j}^{1} 3 x_{k}^{8} x_{\ell}^{7}, x_{i}^{3} x_{j}^{12} x_{k}^{9} x_{\ell}^{7}, 1 \leq j<k \leq 5,1 \leq i, \ell \leq 5, i \neq \ell, i, \ell \neq j, k ;$

II) $x_{i}^{15} x_{j}^{14} x_{k} x_{\ell}, x_{i}^{3} x_{j}^{2} x_{k}^{13} x_{\ell}^{13}, x_{i}^{15} x_{j}^{2} x_{k}^{5} x_{\ell}^{9}, x_{i}^{13} x_{j}^{2} x_{k}^{7} x_{\ell}^{9}, x_{i}^{13} x_{j}^{7} x_{k}^{2} x_{\ell}^{9}, x_{i}^{15} x_{j}^{3} x_{k}^{4} x_{\ell}^{9}, x_{i}^{15} x_{j}^{3} x_{k}^{5} x_{\ell}^{8}$, $x_{i}^{14} x_{j}^{3} x_{k}^{5} x_{\ell}^{9}, x_{i}^{13} x_{j}^{3} x_{k}^{6} x_{\ell}^{9}, x_{i}^{13} x_{j}^{6} x_{k}^{3} x_{\ell}^{9}, 1 \leq j<k<\ell \leq 5,1 \leq i \leq 5, i \neq j, k, \ell ;$

III) $\rho_{(1,5)}\left(x_{1}^{2} x_{2}^{5} x_{3}^{11} x_{4}^{13}\right), \rho_{(1,5)}\left(x_{1}^{6} x_{2}^{11} x_{3}^{5} x_{4}^{9}\right), \rho_{(1,5)}\left(x_{1}^{6} x_{2} x_{3}^{11} x_{4}^{13}\right)$, $\rho_{(1,5)}\left(x_{1}^{6} x_{2}^{11} x_{3} x_{4}^{13}\right), \rho_{(1,5)}\left(x_{1}^{6} x_{2}^{11} x_{3}^{13} x_{4}\right)$.

Lemma 3.3.20. The following monomials are strictly inadmissible:

$$
\begin{aligned}
& Z_{1}=x_{1}^{3} x_{2}^{3} x_{3}^{12} x_{4}^{5} x_{5}^{24}, \\
& Z_{5}=x_{1} x_{2}^{7} x_{3}^{2} x_{4}^{12} x_{5}^{25} \text {, } \\
& Z_{9}=x_{1}^{3} x_{2}^{7} x_{3}^{8} x_{4} x_{5}^{28} \text {, } \\
& Z_{13}=x_{1} x_{2}^{7} x_{3}^{26} x_{4}^{4} x_{5}^{9} \text {, } \\
& Z_{2}=x_{1}^{7} x_{2} x_{3}^{11} x_{4}^{20} x_{5}^{8}, \\
& Z_{6}=x_{1}^{7} x_{2} x_{3}^{2} x_{4}^{12} x_{5}^{25} \text {, } \\
& Z_{10}=x_{1}^{7} x_{2}^{3} x_{3}^{8} x_{4} x_{5}^{28} \text {, } \\
& Z_{14}=x_{1}^{7} x_{2} x_{3}^{26} x_{4}^{4} x_{5}^{9} \text {, } \\
& Z_{3}=x_{1}^{7} x_{2} x_{3}^{2} x_{4}^{28} x_{5}^{9}, \\
& Z_{7}=x_{1} x_{2}^{6} x_{3}^{3} x_{4}^{8} x_{5}^{29} \text {, } \\
& Z_{4}=x_{1} x_{2}^{2} x_{3}^{7} x_{4}^{12} x_{5}^{25}, \\
& Z_{11}=x_{1}^{3} x_{2}^{7} x_{3}^{24} x_{4} x_{5}^{12} \text {, } \\
& Z_{8}=x_{1} x_{2}^{6} x_{3}^{3} x_{4}^{24} x_{5}^{13} \text {, } \\
& Z_{17}=x_{1} x_{2}^{7} x_{3}^{26} x_{4}^{5} x_{5}^{8} \text {, } \\
& Z_{18}=x_{1}^{7} x_{2} x_{3}^{26} x_{4}^{5} x_{5}^{8} \text {, } \\
& Z_{21}=x_{1} x_{2}^{6} x_{3}^{11} x_{4}^{13} x_{5}^{16} \text {, } \\
& Z_{22}=x_{1} x_{2}^{7} x_{3}^{10} x_{4}^{21} x_{5}^{8} \text {, } \\
& Z_{15}=x_{1} x_{2}^{7} x_{3}^{10} x_{4}^{4} x_{5}^{25} \text {, } \\
& Z_{12}=x_{1}^{7} x_{2}^{3} x_{3}^{24} x_{4} x_{5}^{12} \text {, } \\
& Z_{19}=x_{1} x_{2}^{7} x_{3}^{10} x_{4}^{5} x_{5}^{24} \text {, } \\
& Z_{16}=x_{1}^{7} x_{2} x_{3}^{10} x_{4}^{4} x_{5}^{25} \text {, } \\
& Z_{25}=x_{1} x_{2}^{2} x_{3}^{7} x_{4}^{28} x_{5}^{9} \text {, } \\
& Z_{26}=x_{1}^{7} x_{2}^{11} x_{3} x_{4}^{20} x_{5}^{8} \text {, } \\
& Z_{23}=x_{1}^{7} x_{2} x_{3}^{10} x_{4}^{21} x_{5}^{8} \text {, } \\
& Z_{20}=x_{1}^{7} x_{2} x_{3}^{10} x_{4}^{5} x_{5}^{24} \text {, } \\
& Z_{29}=x_{1} x_{2}^{7} x_{3}^{10} x_{4}^{13} x_{5}^{16} \text {, } \\
& Z_{33}=x_{1}^{7} x_{2}^{11} x_{3} x_{4}^{12} x_{5}^{16} \text {, } \\
& Z_{30}=x_{1}^{7} x_{2} x_{3}^{10} x_{4}^{13} x_{5}^{16} \text {, } \\
& Z_{27}=x_{1} x_{2}^{7} x_{3}^{10} x_{4}^{20} x_{5}^{9} \text {, } \\
& Z_{24}=x_{1} x_{2}^{7} x_{3}^{11} x_{4}^{20} x_{5}^{8} \text {, } \\
& Z_{31}=x_{1} x_{2}^{7} x_{3}^{11} x_{4}^{12} x_{5}^{16} \text {, } \\
& Z_{34}=x_{1}^{3} x_{2}^{3} x_{3}^{4} x_{4}^{28} x_{5}^{9} \text {, } \\
& Z_{35}=x_{1}^{3} x_{2}^{3} x_{3}^{28} x_{4}^{4} x_{5}^{9} \text {, } \\
& Z_{28}=x_{1}^{7} x_{2} x_{3}^{10} x_{4}^{20} x_{5}^{9} \text {, } \\
& Z_{32}=x_{1}^{7} x_{2} x_{3}^{11} x_{4}^{12} x_{5}^{16} \text {, } \\
& Z_{36}=x_{1}^{3} x_{2}^{3} x_{3}^{4} x_{4}^{12} x_{5}^{25} \text {, } \\
& Z_{37}=x_{1}^{3} x_{2}^{3} x_{3}^{12} x_{4}^{4} x_{5}^{25}, \\
& Z_{38}=x_{1}^{3} x_{2}^{3} x_{3}^{28} x_{4}^{5} x_{5}^{8}, \\
& Z_{39}=x_{1} x_{2}^{7} x_{3}^{2} x_{4}^{28} x_{5}^{9}, \\
& Z_{40}=x_{1}^{3} x_{2}^{3} x_{3}^{12} x_{4}^{21} x_{5}^{8} \text {. }
\end{aligned}
$$

Proof. We have $\omega\left(Z_{u}\right)=\bar{\omega}_{(1)}, \forall u, 1 \leq u \leq 40$. Consider the monomials $Z_{1}=x_{1}^{3} x_{2}^{3} x_{3}^{12} x_{4}^{5} x_{5}^{24}$ and $Z_{2}=x_{1}^{7} x_{2} x_{3}^{11} x_{4}^{20} x_{5}^{8}$. We prove that these monomials are strictly inadmissible. The others can be proved by a similar technique. Computing the monomials $Z_{1}, Z_{2}$ is long and technical. Indeed, by using Cartan's formula, we get

$$
\begin{aligned}
& Z_{1}=\sum X+S q^{1}\left(\sum \sigma_{1}\right)+S q^{2}\left(\sum \sigma_{2}\right)+S q^{4}\left(\sum \sigma_{4}\right)+S q^{8}\left(\sum \sigma_{8}\right) \operatorname{modulo}\left(\mathscr{P}_{5}^{-}\left(\bar{\omega}_{(1)}\right)\right) \text {, where } \\
& \sum X=x_{1}^{2} x_{2} x_{3}^{3} x_{4}^{12} x_{5}^{29}+x_{1}^{2} x_{2} x_{3}^{3} x_{4}^{13} x_{5}^{28}+x_{1}^{2} x_{2} x_{3}^{5} x_{4}^{12} x_{5}^{27}+x_{1}^{2} x_{2} x_{3}^{5} x_{4}^{13} x_{5}^{26}+x_{1}^{2} x_{2} x_{3}^{5} x_{4}^{24} x_{5}^{15} \\
& +x_{1}^{2} x_{2} x_{3}^{5} x_{4}^{25} x_{5}^{14}+x_{1}^{2} x_{2} x_{3}^{7} x_{4}^{9} x_{5}^{28}+x_{1}^{2} x_{2} x_{3}^{7} x_{4}^{13} x_{5}^{24}+x_{1}^{2} x_{2} x_{3}^{12} x_{4}^{3} x_{5}^{29}+x_{1}^{2} x_{2} x_{3}^{12} x_{4}^{5} x_{5}^{27} \\
& +x_{1}^{2} x_{2} x_{3}^{13} x_{4}^{7} x_{5}^{24}+x_{1}^{2} x_{2} x_{3}^{13} x_{4}^{9} x_{5}^{22}+x_{1}^{2} x_{2} x_{3}^{13} x_{4}^{16} x_{5}^{15}+x_{1}^{2} x_{2} x_{3}^{13} x_{4}^{17} x_{5}^{14}+x_{1}^{2} x_{2} x_{3}^{16} x_{4}^{13} x_{5}^{15} \\
& +x_{1}^{2} x_{2} x_{3}^{24} x_{4}^{5} x_{5}^{15}+x_{1}^{2} x_{2}^{3} x_{3}^{5} x_{4}^{9} x_{5}^{28}+x_{1}^{2} x_{2}^{3} x_{3}^{5} x_{4}^{12} x_{5}^{25}+x_{1}^{2} x_{2}^{3} x_{3}^{9} x_{4}^{5} x_{5}^{28}+x_{1}^{2} x_{2}^{3} x_{3}^{12} x_{4}^{5} x_{5}^{25} \\
& +x_{1}^{2} x_{2}^{5} x_{3}^{3} x_{4}^{9} x_{5}^{28}+x_{1}^{2} x_{2}^{5} x_{3}^{3} x_{4}^{12} x_{5}^{25}+x_{1}^{2} x_{2}^{5} x_{3}^{9} x_{4}^{5} x_{5}^{26}+x_{1}^{2} x_{2}^{5} x_{3}^{12} x_{4}^{3} x_{5}^{25}+x_{1}^{3} x_{2} x_{3}^{3} x_{4}^{12} x_{5}^{28} \\
& +x_{1}^{3} x_{2} x_{3}^{4} x_{4}^{12} x_{5}^{27}+x_{1}^{3} x_{2} x_{3}^{4} x_{4}^{13} x_{5}^{26}+x_{1}^{3} x_{2} x_{3}^{4} x_{4}^{24} x_{5}^{15}+x_{1}^{3} x_{2} x_{3}^{4} x_{4}^{25} x_{5}^{14}+x_{1}^{3} x_{2} x_{3}^{7} x_{4}^{12} x_{5}^{24} \\
& +x_{1}^{3} x_{2} x_{3}^{7} x_{4}^{12} x_{5}^{24}+x_{1}^{3} x_{2} x_{3}^{9} x_{4}^{12} x_{5}^{22}+x_{1}^{3} x_{2} x_{3}^{12} x_{4}^{3} x_{5}^{28}+x_{1}^{3} x_{2} x_{3}^{12} x_{4}^{4} x_{5}^{27}+x_{1}^{3} x_{2} x_{3}^{12} x_{4}^{7} x_{5}^{24} \\
& +x_{1}^{3} x_{2} x_{3}^{12} x_{4}^{9} x_{5}^{22}+x_{1}^{3} x_{2} x_{3}^{12} x_{4}^{16} x_{5}^{15}+x_{1}^{3} x_{2} x_{3}^{12} x_{4}^{17} x_{5}^{14}+x_{1}^{3} x_{2} x_{3}^{16} x_{4}^{12} x_{5}^{15}+x_{1}^{3} x_{2} x_{3}^{24} x_{4}^{4} x_{5}^{15} \\
& +x_{1}^{3} x_{2}^{2} x_{3}^{5} x_{4}^{9} x_{5}^{28}+x_{1}^{3} x_{2}^{2} x_{3}^{5} x_{4}^{12} x_{5}^{25}+x_{1}^{3} x_{2}^{2} x_{3}^{9} x_{4}^{12} x_{5}^{21}+x_{1}^{3} x_{2}^{2} x_{3}^{12} x_{4}^{5} x_{5}^{25}+x_{1}^{3} x_{2}^{2} x_{3}^{12} x_{4}^{9} x_{5}^{21} \\
& +x_{1}^{3} x_{2}^{3} x_{3}^{4} x_{4}^{9} x_{5}^{28}+x_{1}^{3} x_{2}^{3} x_{3}^{4} x_{4}^{12} x_{5}^{25}+x_{1}^{3} x_{2}^{3} x_{3}^{5} x_{4}^{8} x_{5}^{28}+x_{1}^{3} x_{2}^{3} x_{3}^{5} x_{4}^{12} x_{5}^{24} \\
& \sum \sigma_{1}=x_{1}^{3} x_{2} x_{3}^{3} x_{4}^{12} x_{5}^{27}+x_{1}^{3} x_{2} x_{3}^{3} x_{4}^{13} x_{5}^{26}+x_{1}^{3} x_{2} x_{3}^{3} x_{4}^{16} x_{5}^{23}+x_{1}^{3} x_{2} x_{3}^{3} x_{4}^{17} x_{5}^{22}+x_{1}^{3} x_{2} x_{3}^{3} x_{4}^{20} x_{5}^{19} \\
& +x_{1}^{3} x_{2} x_{3}^{3} x_{4}^{21} x_{5}^{18}+x_{1}^{3} x_{2} x_{3}^{3} x_{4}^{24} x_{5}^{15}+x_{1}^{3} x_{2} x_{3}^{3} x_{4}^{25} x_{5}^{14}+x_{1}^{3} x_{2} x_{3}^{7} x_{4}^{9} x_{5}^{26}+x_{1}^{3} x_{2} x_{3}^{7} x_{4}^{11} x_{5}^{24} \\
& +x_{1}^{3} x_{2} x_{3}^{7} x_{4}^{16} x_{5}^{19}+x_{1}^{3} x_{2} x_{3}^{7} x_{4}^{17} x_{5}^{18}+x_{1}^{3} x_{2} x_{3}^{9} x_{4}^{13} x_{5}^{20}+x_{1}^{3} x_{2} x_{3}^{11} x_{4}^{7} x_{5}^{24}+x_{1}^{3} x_{2} x_{3}^{11} x_{4}^{9} x_{5}^{22} \\
& +x_{1}^{3} x_{2} x_{3}^{11} x_{4}^{16} x_{5}^{15}+x_{1}^{3} x_{2} x_{3}^{11} x_{4}^{17} x_{5}^{14}+x_{1}^{3} x_{2} x_{3}^{12} x_{4}^{3} x_{5}^{27}+x_{1}^{3} x_{2} x_{3}^{16} x_{4}^{3} x_{5}^{23}+x_{1}^{3} x_{2} x_{3}^{16} x_{4}^{7} x_{5}^{19} \\
& +x_{1}^{3} x_{2} x_{3}^{16} x_{4}^{11} x_{5}^{15}+x_{1}^{3} x_{2} x_{3}^{20} x_{4}^{3} x_{5}^{19}+x_{1}^{3} x_{2} x_{3}^{24} x_{4}^{3} x_{5}^{15}+x_{1}^{3} x_{2}^{3} x_{3}^{3} x_{4}^{5} x_{5}^{32}+x_{1}^{3} x_{2}^{3} x_{3}^{3} x_{4}^{9} x_{5}^{28} \\
& +x_{1}^{3} x_{2}^{3} x_{3}^{3} x_{4}^{12} x_{5}^{25}+x_{1}^{3} x_{2}^{3} x_{3}^{3} x_{4}^{16} x_{5}^{21}+x_{1}^{3} x_{2}^{3} x_{3}^{9} x_{4}^{5} x_{5}^{26}+x_{1}^{3} x_{2}^{3} x_{3}^{9} x_{4}^{9} x_{5}^{22}+x_{1}^{3} x_{2}^{3} x_{3}^{12} x_{4}^{3} x_{5}^{25} \\
& +x_{1}^{3} x_{2}^{3} x_{3}^{16} x_{4}^{3} x_{5}^{21}+x_{1}^{3} x_{2}^{4} x_{3}^{9} x_{4}^{9} x_{5}^{21}, \\
& \sum \sigma_{2}=x_{1}^{2} x_{2} x_{3}^{3} x_{4}^{12} x_{5}^{27}+x_{1}^{2} x_{2} x_{3}^{3} x_{4}^{13} x_{5}^{26}+x_{1}^{2} x_{2} x_{3}^{3} x_{4}^{24} x_{5}^{15}+x_{1}^{2} x_{2} x_{3}^{3} x_{4}^{25} x_{5}^{14}+x_{1}^{2} x_{2} x_{3}^{7} x_{4}^{9} x_{5}^{26} \\
& +x_{1}^{2} x_{2} x_{3}^{7} x_{4}^{11} x_{5}^{24}+x_{1}^{2} x_{2} x_{3}^{11} x_{4}^{7} x_{5}^{24}+x_{1}^{2} x_{2} x_{3}^{11} x_{4}^{9} x_{5}^{22}+x_{1}^{2} x_{2} x_{3}^{11} x_{4}^{16} x_{5}^{15}+x_{1}^{2} x_{2} x_{3}^{11} x_{4}^{17} x_{5}^{14} \\
& +x_{1}^{2} x_{2} x_{3}^{12} x_{4}^{3} x_{5}^{27}+x_{1}^{2} x_{2} x_{3}^{16} x_{4}^{11} x_{5}^{15}+x_{1}^{2} x_{2} x_{3}^{24} x_{4}^{3} x_{5}^{15}+x_{1}^{2} x_{2}^{3} x_{3}^{3} x_{4}^{9} x_{5}^{28}+x_{1}^{2} x_{2}^{3} x_{3}^{3} x_{4}^{12} x_{5}^{25} \\
& +x_{1}^{2} x_{2}^{3} x_{3}^{9} x_{4}^{5} x_{5}^{26}+x_{1}^{2} x_{2}^{3} x_{3}^{9} x_{4}^{9} x_{5}^{22}+x_{1}^{2} x_{2}^{3} x_{3}^{12} x_{4}^{3} x_{5}^{25}+x_{1}^{5} x_{2} x_{3}^{3} x_{4}^{14} x_{5}^{22}+x_{1}^{5} x_{2} x_{3}^{3} x_{4}^{22} x_{5}^{14} \\
& +x_{1}^{5} x_{2} x_{3}^{7} x_{4}^{10} x_{5}^{22}+x_{1}^{5} x_{2} x_{3}^{7} x_{4}^{18} x_{5}^{14}+x_{1}^{5} x_{2}^{2} x_{3}^{3} x_{4}^{9} x_{5}^{26}+x_{1}^{5} x_{2}^{2} x_{3}^{3} x_{4}^{11} x_{5}^{24}+x_{1}^{5} x_{2}^{2} x_{3}^{3} x_{4}^{12} x_{5}^{23} \\
& +x_{1}^{5} x_{2}^{2} x_{3}^{3} x_{4}^{13} x_{5}^{22}+x_{1}^{5} x_{2}^{2} x_{3}^{3} x_{4}^{20} x_{5}^{15}+x_{1}^{5} x_{2}^{2} x_{3}^{3} x_{4}^{21} x_{5}^{14}+x_{1}^{5} x_{2}^{2} x_{3}^{7} x_{4}^{7} x_{5}^{24}+x_{1}^{5} x_{2}^{2} x_{3}^{7} x_{4}^{9} x_{5}^{22} \\
& +x_{1}^{5} x_{2}^{2} x_{3}^{7} x_{4}^{16} x_{5}^{15}+x_{1}^{5} x_{2}^{2} x_{3}^{7} x_{4}^{17} x_{5}^{14}+x_{1}^{5} x_{2}^{2} x_{3}^{9} x_{4}^{10} x_{5}^{19}+x_{1}^{5} x_{2}^{2} x_{3}^{9} x_{4}^{11} x_{5}^{18}+x_{1}^{5} x_{2}^{2} x_{3}^{10} x_{4}^{9} x_{5}^{19} \\
& +x_{1}^{5} x_{2}^{2} x_{3}^{12} x_{4}^{3} x_{5}^{23}+x_{1}^{5} x_{2}^{2} x_{3}^{16} x_{4}^{7} x_{5}^{15}+x_{1}^{5} x_{2}^{2} x_{3}^{20} x_{4}^{3} x_{5}^{15}+x_{1}^{5} x_{2}^{3} x_{3}^{3} x_{4}^{6} x_{5}^{28}+x_{1}^{5} x_{2}^{3} x_{3}^{3} x_{4}^{8} x_{5}^{26} \\
& +x_{1}^{5} x_{2}^{3} x_{3}^{3} x_{4}^{10} x_{5}^{24}+x_{1}^{5} x_{2}^{3} x_{3}^{3} x_{4}^{12} x_{5}^{22}+x_{1}^{5} x_{2}^{3} x_{3}^{5} x_{4}^{6} x_{5}^{26}+x_{1}^{5} x_{2}^{3} x_{3}^{5} x_{4}^{10} x_{5}^{22}+x_{1}^{5} x_{2}^{3} x_{3}^{10} x_{4}^{3} x_{5}^{24} \\
& +x_{1}^{5} x_{2}^{3} x_{3}^{10} x_{4}^{5} x_{5}^{22}+x_{1}^{5} x_{2}^{3} x_{3}^{12} x_{4}^{3} x_{5}^{22} \text {, }
\end{aligned}
$$




$$
\begin{aligned}
\sum \sigma_{4}= & x_{1}^{3} x_{2} x_{3}^{3} x_{4}^{14} x_{5}^{22}+x_{1}^{3} x_{2} x_{3}^{3} x_{4}^{22} x_{5}^{14}+x_{1}^{3} x_{2} x_{3}^{5} x_{4}^{14} x_{5}^{20}+x_{1}^{3} x_{2} x_{3}^{5} x_{4}^{20} x_{5}^{14}+x_{1}^{3} x_{2} x_{3}^{6} x_{4}^{13} x_{5}^{20} \\
& +x_{1}^{3} x_{2} x_{3}^{7} x_{4}^{10} x_{5}^{22}+x_{1}^{3} x_{2} x_{3}^{7} x_{4}^{18} x_{5}^{14}+x_{1}^{3} x_{2}^{2} x_{3}^{3} x_{4}^{9} x_{5}^{26}+x_{1}^{3} x_{2}^{2} x_{3}^{3} x_{4}^{11} x_{5}^{24}+x_{1}^{3} x_{2}^{2} x_{3}^{3} x_{4}^{12} x_{5}^{23} \\
& +x_{1}^{3} x_{2}^{2} x_{3}^{3} x_{4}^{13} x_{5}^{22}+x_{1}^{3} x_{2}^{2} x_{3}^{3} x_{4}^{20} x_{5}^{15}+x_{1}^{3} x_{2}^{2} x_{3}^{3} x_{4}^{21} x_{5}^{14}+x_{1}^{3} x_{2}^{2} x_{3}^{7} x_{4}^{7} x_{5}^{24}+x_{1}^{3} x_{2}^{2} x_{3}^{7} x_{4}^{9} x_{5}^{22} \\
& +x_{1}^{3} x_{2}^{2} x_{3}^{7} x_{4}^{16} x_{5}^{15}+x_{1}^{3} x_{2}^{2} x_{3}^{7} x_{4}^{17} x_{5}^{14}+x_{1}^{3} x_{2}^{2} x_{3}^{9} x_{4}^{10} x_{5}^{19}+x_{1}^{3} x_{2}^{2} x_{3}^{9} x_{4}^{11} x_{5}^{18}+x_{1}^{3} x_{2}^{2} x_{3}^{10} x_{4}^{9} x_{5}^{19} \\
& +x_{1}^{3} x_{2}^{2} x_{3}^{12} x_{4}^{3} x_{5}^{23}+x_{1}^{3} x_{2}^{2} x_{3}^{16} x_{4}^{7} x_{5}^{15}+x_{1}^{3} x_{2}^{2} x_{3}^{20} x_{4}^{3} x_{5}^{15}+x_{1}^{3} x_{2}^{3} x_{3}^{3} x_{4}^{6} x_{5}^{28}+x_{1}^{3} x_{2}^{3} x_{3}^{3} x_{4}^{8} x_{5}^{26} \\
& +x_{1}^{3} x_{2}^{3} x_{3}^{3} x_{4}^{10} x_{5}^{24}+x_{1}^{3} x_{2}^{3} x_{3}^{3} x_{4}^{12} x_{5}^{22}+x_{1}^{3} x_{2}^{3} x_{3}^{5} x_{4}^{6} x_{5}^{26}+x_{1}^{3} x_{2}^{3} x_{3}^{5} x_{4}^{10} x_{5}^{22}+x_{1}^{3} x_{2}^{3} x_{3}^{10} x_{4}^{3} x_{5}^{24} \\
& +x_{1}^{3} x_{2}^{3} x_{3}^{10} x_{4}^{5} x_{5}^{22}+x_{1}^{3} x_{2}^{3} x_{3}^{12} x_{4}^{3} x_{5}^{22}+x_{1}^{3} x_{2}^{8} x_{3}^{5} x_{4}^{5} x_{5}^{22}+x_{1}^{3} x_{2}^{8} x_{3}^{5} x_{4}^{7} x_{5}^{20}+x_{1}^{3} x_{2}^{8} x_{3}^{5} x_{4}^{12} x_{5}^{15} \\
& +x_{1}^{3} x_{2}^{8} x_{3}^{5} x_{4}^{13} x_{5}^{14}+x_{1}^{3} x_{2}^{8} x_{3}^{12} x_{4}^{5} x_{5}^{15} \\
\sum \sigma_{8} & =x_{1}^{3} x_{2}^{4} x_{3}^{5} x_{4}^{5} x_{5}^{22}+x_{1}^{3} x_{2}^{4} x_{3}^{5} x_{4}^{7} x_{5}^{20}+x_{1}^{3} x_{2}^{4} x_{3}^{5} x_{4}^{12} x_{5}^{15}+x_{1}^{3} x_{2}^{4} x_{3}^{5} x_{4}^{13} x_{5}^{14}+x_{1}^{3} x_{2}^{4} x_{3}^{12} x_{4}^{5} x_{5}^{15} .
\end{aligned}
$$

The above relations imply that $Z_{1}$ is strictly inadmissible. Next we prove that $Z_{2}$ is also strictly inadmissible strictly inadmissible. A direct computation shows that

$$
\begin{aligned}
& Z_{2}=\sum Y+S q^{1}\left(\sum \beta_{1}\right)+S q^{2}\left(\sum \beta_{2}\right)+S q^{4}\left(\sum \beta_{4}\right)+S q^{8}\left(\sum \beta_{8}\right) \text { modulo }\left(\mathscr{P}_{5}^{-}\left(\bar{\omega}_{(1)}\right)\right) \text {, where } \\
& \sum Y=x_{1}^{3} x_{2} x_{3}^{7} x_{4}^{12} x_{5}^{24}+x_{1}^{3} x_{2} x_{3}^{7} x_{4}^{28} x_{5}^{8}+x_{1}^{3} x_{2} x_{3}^{9} x_{4}^{20} x_{5}^{14}+x_{1}^{3} x_{2} x_{3}^{9} x_{4}^{28} x_{5}^{6}+x_{1}^{3} x_{2} x_{3}^{11} x_{4}^{20} x_{5}^{12} \\
& +x_{1}^{3} x_{2} x_{3}^{12} x_{4}^{17} x_{5}^{14}+x_{1}^{3} x_{2} x_{3}^{12} x_{4}^{19} x_{5}^{12}+x_{1}^{3} x_{2} x_{3}^{12} x_{4}^{21} x_{5}^{10}+x_{1}^{3} x_{2} x_{3}^{14} x_{4}^{17} x_{5}^{12}+x_{1}^{3} x_{2} x_{3}^{17} x_{4}^{12} x_{5}^{14} \\
& +x_{1}^{3} x_{2} x_{3}^{20} x_{4}^{9} x_{5}^{14}+x_{1}^{3} x_{2}^{2} x_{3}^{13} x_{4}^{13} x_{5}^{16}+x_{1}^{3} x_{2}^{2} x_{3}^{13} x_{4}^{21} x_{5}^{8}+x_{1}^{3} x_{2}^{4} x_{3}^{7} x_{4}^{25} x_{5}^{8}+x_{1}^{3} x_{2}^{4} x_{3}^{9} x_{4}^{11} x_{5}^{20} \\
& +x_{1}^{3} x_{2}^{4} x_{3}^{9} x_{4}^{21} x_{5}^{10}+x_{1}^{3} x_{2}^{4} x_{3}^{9} x_{4}^{25} x_{5}^{6}+x_{1}^{3} x_{2}^{4} x_{3}^{11} x_{4}^{12} x_{5}^{17}+x_{1}^{3} x_{2}^{4} x_{3}^{11} x_{4}^{20} x_{5}^{9}+x_{1}^{3} x_{2}^{4} x_{3}^{12} x_{4}^{11} x_{5}^{17} \\
& +x_{1}^{3} x_{2}^{4} x_{3}^{12} x_{4}^{19} x_{5}^{9}+x_{1}^{4} x_{2} x_{3}^{7} x_{4}^{11} x_{5}^{24}+x_{1}^{4} x_{2} x_{3}^{11} x_{4}^{17} x_{5}^{14}+x_{1}^{4} x_{2} x_{3}^{11} x_{4}^{19} x_{5}^{12}+x_{1}^{4} x_{2} x_{3}^{11} x_{4}^{21} x_{5}^{10} \\
& +x_{1}^{4} x_{2} x_{3}^{17} x_{4}^{11} x_{5}^{14}+x_{1}^{4} x_{2}^{2} x_{3}^{7} x_{4}^{25} x_{5}^{9}+x_{1}^{4} x_{2}^{2} x_{3}^{11} x_{4}^{13} x_{5}^{17}+x_{1}^{4} x_{2}^{2} x_{3}^{11} x_{4}^{21} x_{5}^{9}+x_{1}^{4} x_{2}^{4} x_{3}^{11} x_{4}^{11} x_{5}^{17} \\
& +x_{1}^{4} x_{2}^{4} x_{3}^{11} x_{4}^{19} x_{5}^{9}+x_{1}^{5} x_{2} x_{3}^{7} x_{4}^{24} x_{5}^{10}+x_{1}^{5} x_{2} x_{3}^{11} x_{4}^{12} x_{5}^{18}+x_{1}^{5} x_{2} x_{3}^{11} x_{4}^{14} x_{5}^{16}+x_{1}^{5} x_{2} x_{3}^{11} x_{4}^{20} x_{5}^{10} \\
& +x_{1}^{5} x_{2} x_{3}^{24} x_{4}^{7} x_{5}^{10}+x_{1}^{5} x_{2} x_{3}^{24} x_{4}^{11} x_{5}^{6}+x_{1}^{5} x_{2}^{2} x_{3}^{7} x_{4}^{24} x_{5}^{9}+x_{1}^{5} x_{2}^{2} x_{3}^{11} x_{4}^{12} x_{5}^{17}+x_{1}^{5} x_{2}^{2} x_{3}^{11} x_{4}^{20} x_{5}^{9} \\
& +x_{1}^{5} x_{2}^{8} x_{3}^{7} x_{4}^{17} x_{5}^{10}+x_{1}^{5} x_{2}^{8} x_{3}^{11} x_{4}^{17} x_{5}^{6}+x_{1}^{7} x_{2} x_{3}^{8} x_{4}^{13} x_{5}^{18}+x_{1}^{7} x_{2} x_{3}^{8} x_{4}^{14} x_{5}^{17}+x_{1}^{7} x_{2} x_{3}^{8} x_{4}^{21} x_{5}^{10} \\
& +x_{1}^{7} x_{2} x_{3}^{8} x_{4}^{22} x_{5}^{9}+x_{1}^{7} x_{2} x_{3}^{9} x_{4}^{12} x_{5}^{18}+x_{1}^{7} x_{2} x_{3}^{9} x_{4}^{20} x_{5}^{10}+x_{1}^{7} x_{2} x_{3}^{10} x_{4}^{9} x_{5}^{20}+x_{1}^{7} x_{2} x_{3}^{10} x_{4}^{12} x_{5}^{17} \\
& +x_{1}^{7} x_{2} x_{3}^{10} x_{4}^{13} x_{5}^{16}+x_{1}^{7} x_{2} x_{3}^{10} x_{4}^{17} x_{5}^{12}+x_{1}^{7} x_{2} x_{3}^{10} x_{4}^{20} x_{5}^{9}+x_{1}^{7} x_{2} x_{3}^{10} x_{4}^{21} x_{5}^{8}+x_{1}^{7} x_{2} x_{3}^{11} x_{4}^{16} x_{5}^{12}, \\
& \sum \beta_{1}=x_{1}^{3} x_{2} x_{3}^{7} x_{4}^{11} x_{5}^{24}+x_{1}^{3} x_{2} x_{3}^{11} x_{4}^{17} x_{5}^{14}+x_{1}^{3} x_{2} x_{3}^{11} x_{4}^{19} x_{5}^{12}+x_{1}^{3} x_{2} x_{3}^{11} x_{4}^{21} x_{5}^{10}+x_{1}^{3} x_{2} x_{3}^{17} x_{4}^{11} x_{5}^{14} \\
& +x_{1}^{3} x_{2} x_{3}^{17} x_{4}^{19} x_{5}^{6}+x_{1}^{3} x_{2}^{4} x_{3}^{11} x_{4}^{11} x_{5}^{17}+x_{1}^{3} x_{2}^{4} x_{3}^{11} x_{4}^{19} x_{5}^{9}+x_{1}^{7} x_{2} x_{3}^{9} x_{4}^{17} x_{5}^{12}+x_{1}^{7} x_{2} x_{3}^{11} x_{4}^{17} x_{5}^{10} \\
& +x_{1}^{7} x_{2} x_{3}^{13} x_{4}^{17} x_{5}^{8}+x_{1}^{7} x_{2}^{2} x_{3}^{7} x_{4}^{13} x_{5}^{17}+x_{1}^{7} x_{2}^{2} x_{3}^{7} x_{4}^{21} x_{5}^{9}+x_{1}^{7} x_{2}^{2} x_{3}^{9} x_{4}^{11} x_{5}^{17}+x_{1}^{7} x_{2}^{2} x_{3}^{9} x_{4}^{19} x_{5}^{9} \\
& +x_{1}^{8} x_{2} x_{3}^{7} x_{4}^{13} x_{5}^{17}+x_{1}^{8} x_{2} x_{3}^{7} x_{4}^{21} x_{5}^{9}+x_{1}^{10} x_{2} x_{3}^{7} x_{4}^{11} x_{5}^{17}+x_{1}^{10} x_{2} x_{3}^{7} x_{4}^{19} x_{5}^{9} \text {, } \\
& \sum \beta_{2}=x_{1}^{3} x_{2} x_{3}^{14} x_{4}^{17} x_{5}^{10}+x_{1}^{3} x_{2}^{2} x_{3}^{7} x_{4}^{13} x_{5}^{20}+x_{1}^{3} x_{2}^{2} x_{3}^{7} x_{4}^{21} x_{5}^{12}+x_{1}^{3} x_{2}^{2} x_{3}^{11} x_{4}^{11} x_{5}^{18}+x_{1}^{3} x_{2}^{2} x_{3}^{11} x_{4}^{19} x_{5}^{10} \\
& +x_{1}^{5} x_{2} x_{3}^{7} x_{4}^{18} x_{5}^{14}+x_{1}^{5} x_{2} x_{3}^{7} x_{4}^{26} x_{5}^{6}+x_{1}^{5} x_{2} x_{3}^{18} x_{4}^{7} x_{5}^{14}+x_{1}^{5} x_{2}^{2} x_{3}^{7} x_{4}^{11} x_{5}^{20}+x_{1}^{5} x_{2}^{2} x_{3}^{7} x_{4}^{21} x_{5}^{10} \\
& +x_{1}^{5} x_{2}^{2} x_{3}^{7} x_{4}^{25} x_{5}^{6}+x_{1}^{5} x_{2}^{2} x_{3}^{11} x_{4}^{11} x_{5}^{16}+x_{1}^{5} x_{2}^{2} x_{3}^{11} x_{4}^{13} x_{5}^{14}+x_{1}^{5} x_{2}^{2} x_{3}^{11} x_{4}^{19} x_{5}^{8}+x_{1}^{5} x_{2}^{2} x_{3}^{11} x_{4}^{21} x_{5}^{6} \\
& +x_{1}^{5} x_{2}^{2} x_{3}^{13} x_{4}^{11} x_{5}^{14}+x_{1}^{5} x_{2}^{2} x_{3}^{13} x_{4}^{19} x_{5}^{6}+x_{1}^{5} x_{2}^{4} x_{3}^{7} x_{4}^{11} x_{5}^{18}+x_{1}^{5} x_{2}^{4} x_{3}^{7} x_{4}^{19} x_{5}^{10}+x_{1}^{7} x_{2} x_{3}^{7} x_{4}^{13} x_{5}^{17} \\
& +x_{1}^{7} x_{2} x_{3}^{7} x_{4}^{20} x_{5}^{10}+x_{1}^{7} x_{2} x_{3}^{7} x_{4}^{21} x_{5}^{9}+x_{1}^{7} x_{2} x_{3}^{9} x_{4}^{11} x_{5}^{17}+x_{1}^{7} x_{2} x_{3}^{9} x_{4}^{19} x_{5}^{9}+x_{1}^{7} x_{2} x_{3}^{10} x_{4}^{9} x_{5}^{18} \\
& +x_{1}^{7} x_{2} x_{3}^{10} x_{4}^{11} x_{5}^{16}+x_{1}^{7} x_{2} x_{3}^{10} x_{4}^{19} x_{5}^{8}+x_{1}^{7} x_{2} x_{3}^{11} x_{4}^{18} x_{5}^{8}+x_{1}^{7} x_{2} x_{3}^{13} x_{4}^{14} x_{5}^{10}+x_{1}^{7} x_{2} x_{3}^{14} x_{4}^{17} x_{5}^{6} \\
& +x_{1}^{7} x_{2} x_{3}^{18} x_{4}^{7} x_{5}^{12}+x_{1}^{7} x_{2} x_{3}^{18} x_{4}^{13} x_{5}^{6}+x_{1}^{7} x_{2}^{2} x_{3}^{7} x_{4}^{17} x_{5}^{12}+x_{1}^{7} x_{2}^{2} x_{3}^{9} x_{4}^{17} x_{5}^{10}+x_{1}^{7} x_{2}^{2} x_{3}^{11} x_{4}^{17} x_{5}^{8} \\
& +x_{1}^{7} x_{2}^{2} x_{3}^{13} x_{4}^{17} x_{5}^{6}+x_{1}^{9} x_{2} x_{3}^{7} x_{4}^{11} x_{5}^{17}+x_{1}^{9} x_{2} x_{3}^{7} x_{4}^{19} x_{5}^{9} \text {, } \\
& +x_{1}^{5} x_{2}^{4} x_{3}^{11} x_{4}^{17} x_{5}^{6}+x_{1}^{11} x_{2} x_{3}^{7} x_{4}^{14} x_{5}^{10}+x_{1}^{11} x_{2} x_{3}^{10} x_{4}^{11} x_{5}^{10}+x_{1}^{11} x_{2} x_{3}^{18} x_{4}^{7} x_{5}^{6}+x_{1}^{11} x_{2}^{2} x_{3}^{7} x_{4}^{17} x_{5}^{6} \text {, } \\
& +x_{1}^{7} x_{2} x_{3}^{10} x_{4}^{11} x_{5}^{10}+x_{1}^{7} x_{2} x_{3}^{12} x_{4}^{9} x_{5}^{10}+x_{1}^{7} x_{2} x_{3}^{12} x_{4}^{11} x_{5}^{8}+x_{1}^{7} x_{2} x_{3}^{18} x_{4}^{7} x_{5}^{6}+x_{1}^{7} x_{2}^{2} x_{3}^{7} x_{4}^{17} x_{5}^{6} \text {. }
\end{aligned}
$$

From the above equalities, we conclude that $Z_{2}$ is also strictly inadmissible.

Lemma 3.3.21. The following monomials are strictly inadmissible:

$$
\begin{array}{llll}
d_{1}=x_{1}^{3} x_{2}^{3} x_{3}^{12} x_{4}^{13} x_{5}^{16}, & d_{2}=x_{1}^{3} x_{2}^{3} x_{3}^{13} x_{4}^{12} x_{5}^{16}, & d_{3}=x_{1}^{3} x_{2}^{3} x_{3}^{13} x_{4}^{20} x_{5}^{8}, & d_{4}=x_{1}^{3} x_{2}^{3} x_{3}^{12} x_{4}^{20} x_{5}^{9}, \\
d_{5}=x_{1}^{3} x_{2}^{7} x_{3}^{4} x_{4}^{8} x_{5}^{25}, & d_{6}=x_{1}^{3} x_{2}^{7} x_{3}^{4} x_{4}^{25} x_{5}^{8}, & d_{7}=x_{1}^{7} x_{2}^{3} x_{3}^{4} x_{4}^{8} x_{5}^{25}, & d_{8}=x_{1}^{7} x_{2}^{3} x_{3}^{4} x_{4}^{25} x_{5}^{8}, \\
d_{9}=x_{1}^{3} x_{2}^{7} x_{3}^{4} x_{4}^{24} x_{5}^{9}, & d_{10}=x_{1}^{3} x_{2}^{7} x_{3}^{4} x_{4}^{9} x_{5}^{24}, & d_{11}=x_{1}^{7} x_{2}^{3} x_{3}^{4} x_{4}^{24} x_{5}^{9}, & d_{12}=x_{1}^{7} x_{2}^{3} x_{3}^{4} x_{4}^{9} x_{5}^{24}, \\
d_{13}=x_{1}^{3} x_{2}^{4} x_{3}^{11} x_{4}^{17} x_{5}^{12}, & d_{14}=x_{1}^{3} x_{2}^{4} x_{3}^{11} x_{4}^{16} x_{5}^{13}, & d_{15}=x_{1}^{3} x_{2}^{4} x_{3}^{11} x_{4}^{13} x_{5}^{16}, & d_{16}=x_{1}^{3} x_{2}^{5} x_{3}^{6} x_{4}^{8} x_{5}^{25}, \\
d_{17}=x_{1}^{3} x_{2}^{5} x_{3}^{6} x_{4}^{25} x_{5}^{8}, & d_{18}=x_{1}^{3} x_{2}^{5} x_{3}^{6} x_{4}^{24} x_{5}^{9}, & d_{19}=x_{1}^{3} x_{2}^{5} x_{3}^{6} x_{4}^{9} x_{5}^{24}, & d_{20}=x_{1}^{3} x_{2}^{5} x_{3}^{7} x_{4}^{8} x_{5}^{24}, \\
d_{21}=x_{1}^{3} x_{2}^{5} x_{3}^{7} x_{4}^{24} x_{5}^{8}, & d_{22}=x_{1}^{3} x_{2}^{7} x_{3}^{5} x_{4}^{8} x_{5}^{24}, & d_{23}=x_{1}^{3} x_{2}^{7} x_{3}^{5} x_{4}^{24} x_{5}^{8}, & d_{24}=x_{1}^{7} x_{2}^{3} x_{3}^{5} x_{4}^{8} x_{5}^{24}, \\
d_{25}=x_{1}^{7} x_{2}^{3} x_{3}^{5} x_{4}^{24} x_{5}^{8}, & d_{26}=x_{1}^{3} x_{2}^{5} x_{3}^{10} x_{4}^{17} x_{5}^{12}, & d_{27}=x_{1}^{3} x_{2}^{5} x_{3}^{10} x_{4}^{16} x_{5}^{13}, & d_{28}=x_{1}^{3} x_{2}^{5} x_{3}^{11} x_{4}^{16} x_{5}^{12}, \\
d_{29}=x_{1}^{3} x_{2}^{7} x_{3}^{8} x_{4}^{17} x_{5}^{12}, & d_{30}=x_{1}^{7} x_{2}^{3} x_{3}^{8} x_{4}^{17} x_{5}^{12}, & d_{31}=x_{1}^{3} x_{2}^{7} x_{3}^{8} x_{4}^{16} x_{5}^{13}, & d_{32}=x_{1}^{3} x_{2}^{7} x_{3}^{8} x_{4}^{13} x_{5}^{16}, \\
d_{33}=x_{1}^{7} x_{2}^{3} x_{3}^{8} x_{4}^{16} x_{5}^{13}, & d_{34}=x_{1}^{7} x_{2}^{3} x_{3}^{8} x_{4}^{13} x_{5}^{16}, & d_{35}=x_{1}^{3} x_{2}^{7} x_{3}^{9} x_{4}^{16} x_{5}^{12}, & d_{36}=x_{1}^{7} x_{2}^{3} x_{3}^{9} x_{4}^{16} x_{5}^{12}, \\
d_{37}=x_{1}^{7} x_{2}^{11} x_{3}^{17} x_{4}^{4} x_{5}^{8} . & & &
\end{array}
$$


Proof. Note that the weight vector of $d_{i}$ is $\bar{\omega}_{(1)}$ for $i=1,2, \ldots, 37$. We prove the lemma for the monomial $d_{1}=x_{1}^{3} x_{2}^{3} x_{3}^{12} x_{4}^{13} x_{5}^{16}$. The others can be proved by an argument similar to the proof of Lemma 3.3.20. By a direct computation, we have

$$
\begin{aligned}
& d_{1}=x_{1}^{2} x_{2} x_{3}^{3} x_{4}^{13} x_{5}^{28}+x_{1}^{2} x_{2} x_{3}^{3} x_{4}^{28} x_{5}^{13}+x_{1}^{2} x_{2} x_{3}^{5} x_{4}^{13} x_{5}^{26}+x_{1}^{2} x_{2} x_{3}^{5} x_{4}^{14} x_{5}^{25}+x_{1}^{2} x_{2} x_{3}^{5} x_{4}^{25} x_{5}^{14} \\
& +x_{1}^{2} x_{2} x_{3}^{5} x_{4}^{26} x_{5}^{13}+x_{1}^{2} x_{2} x_{3}^{10} x_{4}^{13} x_{5}^{21}+x_{1}^{2} x_{2} x_{3}^{12} x_{4}^{7} x_{5}^{25}+x_{1}^{2} x_{2} x_{3}^{12} x_{4}^{11} x_{5}^{21}+x_{1}^{2} x_{2} x_{3}^{13} x_{4}^{14} x_{5}^{17} \\
& +x_{1}^{2} x_{2} x_{3}^{13} x_{4}^{17} x_{5}^{14}+x_{1}^{2} x_{2} x_{3}^{18} x_{4}^{13} x_{5}^{13}+x_{1}^{2} x_{2} x_{3}^{20} x_{4}^{11} x_{5}^{13}+x_{1}^{2} x_{2}^{3} x_{3}^{5} x_{4}^{13} x_{5}^{24}+x_{1}^{2} x_{2}^{3} x_{3}^{5} x_{4}^{24} x_{5}^{13} \\
& +x_{1}^{2} x_{2}^{3} x_{3}^{9} x_{4}^{13} x_{5}^{20}+x_{1}^{2} x_{2}^{3} x_{3}^{12} x_{4}^{13} x_{5}^{17}+x_{1}^{2} x_{2}^{3} x_{3}^{16} x_{4}^{13} x_{5}^{13}+x_{1}^{2} x_{2}^{5} x_{3}^{3} x_{4}^{13} x_{5}^{24}+x_{1}^{2} x_{2}^{5} x_{3}^{3} x_{4}^{24} x_{5}^{13} \\
& +x_{1}^{2} x_{2}^{5} x_{3}^{9} x_{4}^{13} x_{5}^{18}+x_{1}^{2} x_{2}^{5} x_{3}^{9} x_{4}^{17} x_{5}^{14}+x_{1}^{2} x_{2}^{5} x_{3}^{12} x_{4}^{11} x_{5}^{17}+x_{1}^{2} x_{2}^{5} x_{3}^{16} x_{4}^{11} x_{5}^{13}+x_{1}^{3} x_{2} x_{3}^{4} x_{4}^{13} x_{5}^{26} \\
& +x_{1}^{3} x_{2} x_{3}^{4} x_{4}^{14} x_{5}^{25}+x_{1}^{3} x_{2} x_{3}^{4} x_{4}^{25} x_{5}^{14}+x_{1}^{3} x_{2} x_{3}^{4} x_{4}^{26} x_{5}^{13}+x_{1}^{3} x_{2} x_{3}^{9} x_{4}^{14} x_{5}^{20}+x_{1}^{3} x_{2} x_{3}^{9} x_{4}^{20} x_{5}^{14} \\
& +x_{1}^{3} x_{2} x_{3}^{10} x_{4}^{12} x_{5}^{21}+x_{1}^{3} x_{2} x_{3}^{12} x_{4}^{7} x_{5}^{24}+x_{1}^{3} x_{2} x_{3}^{12} x_{4}^{9} x_{5}^{22}+x_{1}^{3} x_{2} x_{3}^{12} x_{4}^{14} x_{5}^{17}+x_{1}^{3} x_{2} x_{3}^{12} x_{4}^{17} x_{5}^{14} \\
& +x_{1}^{3} x_{2} x_{3}^{18} x_{4}^{12} x_{5}^{13}+x_{1}^{3} x_{2} x_{3}^{20} x_{4}^{9} x_{5}^{14}+x_{1}^{3} x_{2}^{2} x_{3}^{5} x_{4}^{13} x_{5}^{24}+x_{1}^{3} x_{2}^{2} x_{3}^{5} x_{4}^{24} x_{5}^{13}+x_{1}^{3} x_{2}^{2} x_{3}^{12} x_{4}^{17} x_{5}^{13} \\
& +x_{1}^{3} x_{2}^{3} x_{3}^{4} x_{4}^{13} x_{5}^{24}+x_{1}^{3} x_{2}^{3} x_{3}^{8} x_{4}^{12} x_{5}^{21}+x_{1}^{3} x_{2}^{3} x_{3}^{12} x_{4}^{12} x_{5}^{17} \\
& +S q^{1}\left(\sum X\right)+S q^{2}\left(\sum Y\right)+S q^{4}\left(\sum Z\right)+S q^{8}\left(\sum T\right) \operatorname{modulo}\left(\mathscr{P}_{5}^{-}\left(\bar{\omega}_{(1)}\right)\right) \text {, where } \\
& \sum X=x_{1}^{3} x_{2} x_{3}^{3} x_{4}^{13} x_{5}^{26}+x_{1}^{3} x_{2} x_{3}^{3} x_{4}^{14} x_{5}^{25}+x_{1}^{3} x_{2} x_{3}^{3} x_{4}^{17} x_{5}^{22}+x_{1}^{3} x_{2} x_{3}^{3} x_{4}^{18} x_{5}^{21}+x_{1}^{3} x_{2} x_{3}^{3} x_{4}^{21} x_{5}^{18} \\
& +x_{1}^{3} x_{2} x_{3}^{3} x_{4}^{22} x_{5}^{17}+x_{1}^{3} x_{2} x_{3}^{3} x_{4}^{25} x_{5}^{14}+x_{1}^{3} x_{2} x_{3}^{3} x_{4}^{26} x_{5}^{13}+x_{1}^{3} x_{2} x_{3}^{7} x_{4}^{17} x_{5}^{18}+x_{1}^{3} x_{2} x_{3}^{7} x_{4}^{18} x_{5}^{17} \\
& +x_{1}^{3} x_{2} x_{3}^{10} x_{4}^{7} x_{5}^{25}+x_{1}^{3} x_{2} x_{3}^{10} x_{4}^{11} x_{5}^{21}+x_{1}^{3} x_{2} x_{3}^{11} x_{4}^{14} x_{5}^{17}+x_{1}^{3} x_{2} x_{3}^{11} x_{4}^{17} x_{5}^{14}+x_{1}^{3} x_{2} x_{3}^{18} x_{4}^{7} x_{5}^{17} \\
& +x_{1}^{3} x_{2} x_{3}^{18} x_{4}^{11} x_{5}^{13}+x_{1}^{3} x_{2}^{3} x_{3}^{3} x_{4}^{13} x_{5}^{24}+x_{1}^{3} x_{2}^{3} x_{3}^{3} x_{4}^{17} x_{5}^{20}+x_{1}^{3} x_{2}^{3} x_{3}^{3} x_{4}^{20} x_{5}^{17}+x_{1}^{3} x_{2}^{3} x_{3}^{3} x_{4}^{24} x_{5}^{13} \\
& +x_{1}^{3} x_{2}^{3} x_{3}^{9} x_{4}^{13} x_{5}^{18}+x_{1}^{3} x_{2}^{3} x_{3}^{9} x_{4}^{17} x_{5}^{14}+x_{1}^{3} x_{2}^{3} x_{3}^{12} x_{4}^{11} x_{5}^{17}+x_{1}^{3} x_{2}^{3} x_{3}^{16} x_{4}^{11} x_{5}^{13}+x_{1}^{3} x_{2}^{4} x_{3}^{9} x_{4}^{17} x_{5}^{13}, \\
& \sum Y=x_{1}^{2} x_{2} x_{3}^{3} x_{4}^{13} x_{5}^{26}+x_{1}^{2} x_{2} x_{3}^{3} x_{4}^{14} x_{5}^{25}+x_{1}^{2} x_{2} x_{3}^{3} x_{4}^{25} x_{5}^{14}+x_{1}^{2} x_{2} x_{3}^{3} x_{4}^{26} x_{5}^{13}+x_{1}^{2} x_{2} x_{3}^{10} x_{4}^{7} x_{5}^{25} \\
& +x_{1}^{2} x_{2} x_{3}^{10} x_{4}^{11} x_{5}^{21}+x_{1}^{2} x_{2} x_{3}^{11} x_{4}^{14} x_{5}^{17}+x_{1}^{2} x_{2} x_{3}^{11} x_{4}^{17} x_{5}^{14}+x_{1}^{2} x_{2} x_{3}^{18} x_{4}^{11} x_{5}^{13}+x_{1}^{2} x_{2}^{3} x_{3}^{3} x_{4}^{13} x_{5}^{24} \\
& +x_{1}^{2} x_{2}^{3} x_{3}^{3} x_{4}^{24} x_{5}^{13}+x_{1}^{2} x_{2}^{3} x_{3}^{9} x_{4}^{13} x_{5}^{18}+x_{1}^{2} x_{2}^{3} x_{3}^{9} x_{4}^{17} x_{5}^{14}+x_{1}^{2} x_{2}^{3} x_{3}^{12} x_{4}^{11} x_{5}^{17}+x_{1}^{2} x_{2}^{3} x_{3}^{16} x_{4}^{11} x_{5}^{13} \\
& +x_{1}^{5} x_{2} x_{3}^{7} x_{4}^{14} x_{5}^{18}+x_{1}^{5} x_{2} x_{3}^{7} x_{4}^{18} x_{5}^{14}+x_{1}^{5} x_{2} x_{3}^{10} x_{4}^{7} x_{5}^{22}+x_{1}^{5} x_{2} x_{3}^{18} x_{4}^{7} x_{5}^{14}+x_{1}^{5} x_{2}^{2} x_{3}^{3} x_{4}^{13} x_{5}^{22} \\
& +x_{1}^{5} x_{2}^{2} x_{3}^{3} x_{4}^{14} x_{5}^{21}+x_{1}^{5} x_{2}^{2} x_{3}^{3} x_{4}^{21} x_{5}^{14}+x_{1}^{5} x_{2}^{2} x_{3}^{3} x_{4}^{22} x_{5}^{13}+x_{1}^{5} x_{2}^{2} x_{3}^{7} x_{4}^{14} x_{5}^{17}+x_{1}^{5} x_{2}^{2} x_{3}^{7} x_{4}^{17} x_{5}^{14} \\
& +x_{1}^{5} x_{2}^{2} x_{3}^{10} x_{4}^{7} x_{5}^{21}+x_{1}^{5} x_{2}^{2} x_{3}^{18} x_{4}^{7} x_{5}^{13}+x_{1}^{5} x_{2}^{3} x_{3}^{3} x_{4}^{14} x_{5}^{20}+x_{1}^{5} x_{2}^{3} x_{3}^{3} x_{4}^{20} x_{5}^{14}+x_{1}^{5} x_{2}^{3} x_{3}^{5} x_{4}^{14} x_{5}^{18} \\
& +x_{1}^{5} x_{2}^{3} x_{3}^{5} x_{4}^{18} x_{5}^{14}+x_{1}^{5} x_{2}^{3} x_{3}^{10} x_{4}^{11} x_{5}^{16}+x_{1}^{5} x_{2}^{3} x_{3}^{10} x_{4}^{13} x_{5}^{14}+x_{1}^{5} x_{2}^{3} x_{3}^{12} x_{4}^{11} x_{5}^{14}, \\
& \sum Z=x_{1}^{3} x_{2} x_{3}^{7} x_{4}^{14} x_{5}^{18}+x_{1}^{3} x_{2} x_{3}^{7} x_{4}^{18} x_{5}^{14}+x_{1}^{3} x_{2} x_{3}^{18} x_{4}^{7} x_{5}^{14}+x_{1}^{3} x_{2}^{2} x_{3}^{3} x_{4}^{13} x_{5}^{22}+x_{1}^{3} x_{2}^{2} x_{3}^{3} x_{4}^{14} x_{5}^{21} \\
& +x_{1}^{3} x_{2}^{2} x_{3}^{3} x_{4}^{21} x_{5}^{14}+x_{1}^{3} x_{2}^{2} x_{3}^{3} x_{4}^{22} x_{5}^{13}+x_{1}^{3} x_{2}^{2} x_{3}^{7} x_{4}^{14} x_{5}^{17}+x_{1}^{3} x_{2}^{2} x_{3}^{7} x_{4}^{17} x_{5}^{14}+x_{1}^{3} x_{2}^{2} x_{3}^{10} x_{4}^{7} x_{5}^{21} \\
& +x_{1}^{3} x_{2}^{2} x_{3}^{18} x_{4}^{7} x_{5}^{13}+x_{1}^{3} x_{2}^{3} x_{3}^{3} x_{4}^{14} x_{5}^{20}+x_{1}^{3} x_{2}^{3} x_{3}^{3} x_{4}^{20} x_{5}^{14}+x_{1}^{3} x_{2}^{3} x_{3}^{4} x_{4}^{20} x_{5}^{13}+x_{1}^{3} x_{2}^{3} x_{3}^{5} x_{4}^{14} x_{5}^{18} \\
& +x_{1}^{3} x_{2}^{3} x_{3}^{5} x_{4}^{18} x_{5}^{14}+x_{1}^{3} x_{2}^{3} x_{3}^{10} x_{4}^{11} x_{5}^{16}+x_{1}^{3} x_{2}^{3} x_{3}^{10} x_{4}^{13} x_{5}^{14}+x_{1}^{3} x_{2}^{3} x_{3}^{12} x_{4}^{11} x_{5}^{14}+x_{1}^{3} x_{2}^{8} x_{3}^{5} x_{4}^{13} x_{5}^{14} \\
& +x_{1}^{3} x_{2}^{8} x_{3}^{5} x_{4}^{14} x_{5}^{13}+x_{1}^{3} x_{2}^{8} x_{3}^{12} x_{4}^{7} x_{5}^{13}, \\
& \sum T=x_{1}^{3} x_{2}^{2} x_{3}^{12} x_{4}^{9} x_{5}^{13}+x_{1}^{3} x_{2}^{3} x_{3}^{8} x_{4}^{12} x_{5}^{13}+x_{1}^{3} x_{2}^{4} x_{3}^{5} x_{4}^{13} x_{5}^{14} \\
& +x_{1}^{3} x_{2}^{4} x_{3}^{5} x_{4}^{14} x_{5}^{13}+x_{1}^{3} x_{2}^{4} x_{3}^{10} x_{4}^{9} x_{5}^{13}+x_{1}^{3} x_{2}^{4} x_{3}^{12} x_{4}^{7} x_{5}^{13} \text {. }
\end{aligned}
$$

The above relations show that $d_{1}$ is strictly inadmissible. The lemma follows.

Proof of Proposition 3.3.18. Let $a$ be an admissible monomial in $\mathscr{P}_{5}^{+}$such that $\omega(a)=\bar{\omega}_{(1)}$. Then $a=x_{t} x_{k} x_{m} u^{2}$ with $1 \leq t<k<m \leq 5$ and $u \in\left(\mathscr{P}_{5}^{+}\right)_{22}$. Since $a$ is admissible, by Theorem 2.2.1, $u$ is also admissible. Further, $u \in \mathscr{B}_{5}^{+}\left(\omega_{(1)}\right)$.

Let $X \in \mathscr{B}_{5}^{+}\left(\omega_{(1)}\right)$ such that $x_{t} x_{k} x_{m} X^{2} \in\left(\mathscr{P}_{5}^{+}\right)_{47}$. By a direct computation using Proposition 3.3.10, we see that if $x_{t} x_{k} x_{m} X^{2} \neq y_{47, j}, 1 \leq j \leq 370$, then there is a monomial $b$ which is given in one of Lemmas 3.3.16 - 3.3.21 such that $x_{t} x_{k} x_{m} X^{2}=b Y^{2^{r}}$ with suitable monomia $Y \in \mathscr{P}_{5}$, and $r=\max \left\{s \in \mathbb{Z}: \omega_{s}(b)>0\right\}$. By Theorem 2.2.1, $x_{t} x_{k} x_{m} X^{2}$ is inadmissible. On the other hand, we have $a=x_{t} x_{k} x_{m} u^{2}$ and $a$ is admissible, hence $a=y_{j}$ for some $j=1,2, \ldots, 370$. This completes the proof of the proposition.

Remark 3.3.22. For $1 \leq j \leq 370$, we have $\left[y_{j}:=y_{47, j}\right]_{\bar{\omega}_{(1)}} \neq[0]$. Indeed, suppose that there is a linear relation

$$
\mathcal{S}=\sum_{1 \leq j \leq 170} \gamma_{j} y_{j} \equiv_{\bar{\omega}_{(1)}} 0
$$

where $\gamma_{j} \in \mathbb{Z} / 2$, for all $j$. Based on Theorem 3.1.3 and Proposition 3.2.1, for $(k ; \mathscr{K}) \in \mathcal{N}_{5}$, we explicitly compute $\pi_{(k ; \mathscr{K})}(\mathcal{S})$ in terms of a given minimal set of $\mathcal{A}_{2}$-generators in $\mathscr{P}_{4}\left(\operatorname{modulo}\left(\mathcal{A}_{2}^{+} \mathscr{P}_{4}\right)\right)$. By computing from the relations $\pi_{(k ; \mathscr{K})}(\mathcal{S}) \equiv_{\bar{\omega}_{(1)}} 0, \ell(\mathscr{K})=1$, one gets $\gamma_{j}=0, \forall j, 1 \leq j \leq 370$. Note that these computations are similar to the proof of Propositions 3.3.1 and 3.3.10. Combining this and Proposition 3.3.18, we have a direct corollary.

Corollary 3.3.23. The set $\left[\bar{\Phi}^{+}\left(\mathscr{B}_{4}\left(\bar{\omega}_{(1)}\right)\right) \bigcup\left(\bigcup_{1 \leq k \leq 5} \overline{\mathscr{B}}^{+}\left(k, \bar{\omega}_{(1)}\right)\right) \bigcup \mathcal{F}\right]_{\bar{\omega}_{(1)}}$ is a basis of the $\mathbb{Z} / 2$ vector space $Q \mathscr{P}_{5}^{+}\left(\bar{\omega}_{(1)}\right)$. This implies $\operatorname{dim}\left(Q \mathscr{P}_{5}^{+}\left(\bar{\omega}_{(1)}\right)\right)=370$.

Remark 3.3.24. Consider the weight vector $\omega^{* *}=(3,2,4)$ with $\operatorname{deg} \omega^{* *}=23$. By using a result in [69], we see that the following monomials are strictly inadmissible: 


$$
\begin{array}{llll}
e_{1}=x_{1}^{7} x_{2}^{2} x_{3}^{4} x_{4}^{5} x_{5}^{5}, & e_{2}=x_{1}^{7} x_{2}^{2} x_{3}^{5} x_{4}^{4} x_{5}^{5}, & e_{3}=x_{1}^{7} x_{2}^{2} x_{3}^{5} x_{4}^{5} x_{5}^{4}, & e_{4}=x_{1}^{3} x_{2}^{7} x_{3}^{4} x_{4}^{4} x_{5}^{5}, \\
e_{5}=x_{1}^{3} x_{2}^{7} x_{3}^{4} x_{4}^{5} x_{5}^{4}, & e_{6}=x_{1}^{3} x_{2}^{7} x_{3}^{5} x_{4}^{4} x_{5}^{4}, & e_{7}=x_{1}^{7} x_{2}^{3} x_{3}^{4} x_{4}^{4} x_{5}^{5}, & e_{8}=x_{1}^{7} x_{2}^{3} x_{3}^{4} x_{4}^{5} x_{5}^{4}, \\
e_{9}=x_{1}^{7} x_{2}^{3} x_{3}^{5} x_{4}^{4} x_{5}^{4}, & e_{10}=x_{1}^{3} x_{2}^{4} x_{3}^{4} x_{4}^{5} x_{5}^{7}, & e_{11}=x_{1}^{3} x_{2}^{4} x_{3}^{4} x_{4}^{7} x_{5}^{5}, & e_{12}=x_{1}^{3} x_{2}^{4} x_{3}^{5} x_{4}^{4} x_{5}^{7}, \\
e_{13}=x_{1}^{3} x_{2}^{4} x_{3}^{7} x_{4}^{4} x_{5}^{5}, & e_{14}=x_{1}^{3} x_{2}^{4} x_{3}^{5} x_{4}^{7} x_{5}^{4}, & e_{15}=x_{1}^{3} x_{2}^{4} x_{3}^{7} x_{4}^{5} x_{5}^{4}, & e_{16}=x_{1}^{3} x_{2}^{5} x_{3}^{4} x_{4}^{4} x_{5}^{7}, \\
e_{17}=x_{1}^{3} x_{2}^{5} x_{3}^{4} x_{4}^{7} x_{5}^{4}, & e_{18}=x_{1}^{3} x_{2}^{5} x_{3}^{7} x_{4}^{4} x_{5}^{4}, & e_{19}=x_{1}^{3} x_{2}^{6} x_{3}^{4} x_{4}^{5} x_{5}^{5}, & e_{20}=x_{1}^{3} x_{2}^{6} x_{3}^{5} x_{4}^{4} x_{5}^{5}, \\
e_{21}=x_{1}^{3} x_{2}^{6} x_{3}^{5} x_{4}^{5} x_{5}^{4}, & e_{22}=x_{1}^{3} x_{2}^{4} x_{3}^{6} x_{4}^{5} x_{5}^{5}, & e_{23}=x_{1}^{3} x_{2}^{4} x_{3}^{5} x_{4}^{6} x_{5}^{5}, & e_{24}=x_{1}^{3} x_{2}^{4} x_{3}^{5} x_{4}^{5} x_{5}^{6}, \\
e_{25}=x_{1}^{3} x_{2}^{5} x_{3}^{4} x_{4}^{6} x_{5}^{5}, & e_{26}=x_{1}^{3} x_{2}^{5} x_{3}^{6} x_{4}^{4} x_{5}^{5}, & e_{27}=x_{1}^{3} x_{2}^{5} x_{3}^{4} x_{4}^{5} x_{5}^{6}, & e_{28}=x_{1}^{3} x_{2}^{5} x_{3}^{6} x_{4}^{5} x_{5}^{4}, \\
e_{29}=x_{1}^{3} x_{2}^{5} x_{3}^{5} x_{4}^{4} x_{5}^{6}, & e_{30}=x_{1}^{3} x_{2}^{5} x_{3}^{5} x_{4}^{6} x_{5}^{4} . & &
\end{array}
$$

Note that $\omega\left(e_{k}\right)=\omega^{* *}, k=1,2, \ldots, 30$. Let $X \in \mathscr{B}_{5}^{+}(47)$ such that either $\omega(X)=\bar{\omega}_{(2)}$ or $\omega(X)=\bar{\omega}_{(3)}$. Then $X=X_{(\{\ell, m\}, 5)} y^{2}$ with $1 \leq \ell<m \leq 5$, and $y \in\left(\mathscr{P}_{5}^{+}\right)_{22}$. By a direct computation using Theorem 2.2.1, and Proposition 3.3.14(I), (II), we see that either $y \in \mathscr{B}_{5}^{+}\left(\omega_{(2)}\right)$ or $y \in \mathscr{B}_{5}^{+}\left(\omega_{(3)}\right)$ and there is a monomial $Z=e_{i}$ for some $i, 1 \leq i \leq 30$ such that $X=$ $X_{(\{\ell, m\}, 5)} y^{2}=Z u^{2^{t}}, 1 \leq \ell<m \leq 5$, with suitable monomia $u \in \mathscr{P}_{5}$, and $t=\max \{a \in \mathbb{Z}$ : $\left.\omega_{a}(Z)>0\right\}$. By Theorem 2.2.1, we get either $[X]=[0]_{\bar{\omega}_{(2)}}$ or $[X]=[0]_{\bar{\omega}_{(3)}}$. As a consequence, we get the following.

Proposition 3.3.25. The $\mathbb{Z} / 2$-vector spaces $Q \mathscr{P}_{5}^{+}\left(\bar{\omega}_{(2)}\right)$ and $Q \mathscr{P}_{5}^{+}\left(\bar{\omega}_{(3)}\right)$ are trivial.

Lemma 3.3.26. The set $\left\{\left[y_{47, j}\right]_{\bar{\omega}_{(4)}}: 371 \leq j \leq 479\right\}$ is a minimal system of generators for $\left.Q \mathscr{P}_{5}^{+}\left(\bar{\omega}_{(4)}\right)\right)$, where the monomials $y_{47, j}, j=371, \ldots, 479$, are described in Sect.6.7 of the online version [44].

In order to prove the lemma, we need to use some results. As an immediate consequence of a result in [62], we obtain the following.

Lemma 3.3.27. The following monomials are strictly inadmissible:

I) $x_{a}^{7} x_{b}^{2} x_{c}^{15} x_{d}^{7}, x_{a}^{7} x_{b}^{15} x_{c}^{2} x_{d}^{7}, x_{a}^{7} x_{b}^{6} x_{c}^{3} x_{d}^{15}, x_{a}^{7} x_{b}^{3} x_{c}^{6} x_{d}^{15}, x_{a}^{7} x_{b}^{3} x_{c}^{14} x_{d}^{7}$, $x_{a}^{7} x_{b}^{14} x_{c}^{3} x_{d}^{7}, x_{a}^{7} x_{b}^{6} x_{c}^{11} x_{d}^{7}, x_{a}^{7} x_{b}^{11} x_{c}^{6} x_{d}^{7}, 1 \leq b<c \leq 5,1 \leq a, d \leq 5, a \neq d, a, d \neq b, c$.

II) $\rho_{(k, 5)}(v), 1 \leq k \leq 5$, where $v$ is one of the following monomials:

$$
x_{1}^{7} x_{2}^{7} x_{3}^{7} x_{4}^{10}, x_{1}^{7} x_{2}^{7} x_{3}^{10} x_{4}^{7}, x_{1}^{7} x_{2}^{10} x_{3}^{7} x_{4}^{7} .
$$

We now consider the weight vector $\omega^{* * *}=(3,4,3,1)$ with $\operatorname{deg} \omega^{* * *}=31$. The following lemma can be easily proved by a direct computation.

Lemma 3.3.28. All permutations of the following monomials are strictly inadmissible:

$$
\begin{array}{llll}
x_{1} x_{2}^{2} x_{3}^{6} x_{4}^{7} x_{5}^{15}, & x_{1} x_{2}^{2} x_{3}^{1} 4 x_{4}^{7} x_{5}^{7}, & x_{1} x_{2}^{6} x_{3}^{7} x_{4}^{7} x_{5}^{10}, & x_{1}^{7} x_{2}^{2} x_{3}^{2} x_{4}^{5} x_{5}^{15}, \\
x_{1}^{7} x_{2}^{2} x_{3}^{2} x_{4}^{7} x_{5}^{13}, & x_{1}^{3} x_{2}^{2} x_{3}^{4} x_{4}^{7} x_{5}^{15}, & x_{1}^{3} x_{2}^{2} x_{3}^{7} x_{4}^{7} x_{5}^{12}, & x_{1}^{7} x_{2}^{2} x_{3}^{4} x_{4}^{7} x_{5}^{11}, \\
x_{1}^{7} x_{2}^{2} x_{3}^{5} x_{4}^{7} x_{5}^{10}, & x_{1}^{7} x_{2}^{2} x_{3}^{6} x_{4}^{7} x_{5}^{9}, & x_{1}^{7} x_{2}^{2} x_{3}^{7} x_{4}^{7} x_{5}^{8}, & x_{1}^{3} x_{2}^{3} x_{3}^{4} x_{4}^{6} x_{5}^{15}, \\
x_{1}^{3} x_{2}^{3} x_{3}^{4} x_{4}^{7} x_{5}^{14}, & x_{1}^{3} x_{2}^{3} x_{3}^{6} x_{4}^{7} x_{5}^{12}, & x_{1}^{3} x_{2}^{4} x_{3}^{6} x_{4}^{7} x_{5}^{11}, & x_{1}^{3} x_{2}^{4} x_{3}^{7} x_{4}^{7} x_{5}^{10}, \\
x_{1}^{3} x_{2}^{6} x_{3}^{7} x_{4}^{7} x_{5}^{8} . & &
\end{array}
$$

Lemma 3.3.29. If $(m, n, p, q, r)$ is a permutation of $(1,2,3,4,5)$, then the following monomials are strictly inadmissible:

$$
\begin{aligned}
& x_{m}^{3} x_{n}^{13} x_{p}^{6} x_{q}^{3} x_{r}^{6} \neq X \quad \in\left\{x_{1}^{3} x_{2}^{3} x_{3}^{13} x_{4}^{6} x_{5}^{6}, x_{1}^{3} x_{2}^{13} x_{3}^{3} x_{4}^{6} x_{5}^{6}\right\}, \\
& x_{m}^{7} x_{n}^{2} x_{p}^{5} x_{q}^{6} x_{r}^{11} \neq Y \quad \in\left\{x_{1}^{7} x_{2}^{11} x_{3}^{5} x_{4}^{2} x_{5}^{6}, x_{1}^{7} x_{2}^{11} x_{3}^{5} x_{4}^{6} x_{5}^{2}\right\}, \\
& x_{m}^{3} x_{n}^{5} x_{p}^{6} x_{q}^{6} x_{r}^{11} \neq Z \in\left.\in x_{1}^{3} x_{2}^{5} x_{3}^{6} x_{4}^{6} x_{5}^{11}, x_{1}^{3} x_{2}^{5} x_{3}^{11} x_{4}^{6} x_{5}^{6}\right\}, \\
& x_{m}^{3} x_{n}^{7} x_{p}^{9} x_{q}^{6} x_{r}^{6} \neq G \quad \in\left\{x_{1}^{3} x_{2}^{7} x_{3}^{9} x_{4}^{6} x_{5}^{6}, x_{1}^{7} x_{2}^{3} x_{3}^{9} x_{4}^{6} x_{5}^{6}, x_{1}^{7} x_{2}^{9} x_{3}^{3} x_{4}^{6} x_{5}^{6}\right\}, \\
& x_{m}^{3} x_{n}^{2} x_{p}^{6} x_{q}^{7} x_{r}^{13} \neq H \in \in x_{1}^{3} x_{2}^{7} x_{3}^{13} x_{4}^{2} x_{5}^{6}, x_{1}^{3} x_{2}^{7} x_{3}^{13} x_{4}^{6} x_{5}^{2}, x_{1}^{7} x_{2}^{3} x_{3}^{13} x_{4}^{2} x_{5}^{6}, \\
&\left.x_{1}^{7} x_{2}^{3} x_{3}^{13} x_{4}^{6} x_{5}^{2}\right\}, \\
& x_{m}^{3} x_{n}^{5} x_{p}^{6} x_{q}^{7} x_{r}^{10} \neq F \quad \in\left\{x_{1}^{3} x_{2}^{5} x_{3}^{6} x_{4}^{7} x_{5}^{10}, x_{1}^{3} x_{2}^{5} x_{3}^{7} x_{4}^{6} x_{5}^{10}, x_{1}^{3} x_{2}^{5} x_{3}^{7} x_{4}^{10} x_{5}^{6},\right. \\
& x_{1}^{3} x_{2}^{7} x_{3}^{5} x_{4}^{6} x_{5}^{10}, x_{1}^{3} x_{2}^{7} x_{3}^{5} x_{4}^{10} x_{5}^{6}, x_{1}^{7} x_{2}^{3} x_{3}^{5} x_{4}^{6} x_{5}^{10}, \\
&\left.x_{1}^{7} x_{2}^{3} x_{3}^{5} x_{4}^{10} x_{5}^{6}\right\}, \\
& x_{m}^{3} x_{n}^{5} x_{p}^{14} x_{q}^{3} x_{r}^{6} \neq T \in x_{1}^{3} x_{2}^{3} x_{3}^{5} x_{4}^{6} x_{5}^{14}, x_{1}^{3} x_{2}^{3} x_{3}^{5} x_{4}^{14} x_{5}^{6}, x_{1}^{3} x_{2}^{5} x_{3}^{3} x_{4}^{6} x_{5}^{14}, \\
&\left.x_{1}^{3} x_{2}^{5} x_{3}^{3} x_{4}^{14} x_{5}^{6}, x_{1}^{3} x_{2}^{5} x_{3}^{6} x_{4}^{3} x_{5}^{14}, x_{1}^{3} x_{2}^{5} x_{3}^{6} x_{4}^{14} x_{5}^{3}\right\} .
\end{aligned}
$$

Proof. It is easy to see that the weight vector of these monomials is $\omega^{* * *}$. Note that the monomials $X, Y, Z, G, H, F$, and $T$ are admissible. We now prove the lemma for the monomials $f=x_{m}^{3} x_{n}^{13} x_{p}^{6} x_{q}^{3} x_{r}^{6}$ and $g=x_{m}^{3} x_{n}^{5} x_{p}^{14} x_{q}^{3} x_{r}^{6}$. The others can be proved by an argument similar to the proofs of Lemmas 3.3.16 and 3.3.17. Applying the Cartan formula, we get

$$
f=x_{m}^{3} x_{n}^{13} x_{p}^{3} x_{q}^{6} x_{r}^{6}+S q^{1}\left(\sum \bar{A}_{1}\right)+S q^{2}\left(\sum \bar{B}_{1}\right)+S q^{4}\left(\sum \bar{C}_{1}\right) \text { modulo }\left(\mathscr{P}_{5}^{-}\left(\omega^{* * *}\right)\right), \text { where }
$$




$$
\begin{aligned}
\sum \bar{A}_{1}= & x_{m}^{3} x_{n}^{7} x_{p}^{5} x_{q}^{5} x_{r}^{10}+x_{m}^{3} x_{n}^{7} x_{p}^{5} x_{q}^{6} x_{r}^{9}+x_{m}^{3} x_{n}^{7} x_{p}^{6} x_{q}^{5} x_{r}^{9}+x_{m}^{3} x_{n}^{7} x_{p}^{6} x_{q}^{9} x_{r}^{5}+x_{m}^{3} x_{n}^{7} x_{p}^{9} x_{q}^{6} x_{r}^{5} \\
& +x_{m}^{3} x_{n}^{11} x_{p}^{5} x_{q}^{6} x_{r}^{5}+x_{m}^{3} x_{n}^{14} x_{p}^{3} x_{q}^{5} x_{r}^{5}+x_{m}^{6} x_{n}^{11} x_{p}^{5} x_{q}^{3} x_{r}^{5}, \\
\sum \bar{B}_{1}= & x_{m}^{3} x_{n}^{11} x_{p}^{5} x_{q}^{5} x_{r}^{5}+x_{m}^{3} x_{n}^{11} x_{p}^{6} x_{q}^{3} x_{r}^{6}+x_{m}^{3} x_{n}^{13} x_{p}^{5} x_{q}^{3} x_{r}^{5}+x_{m}^{5} x_{n}^{7} x_{p}^{3} x_{q}^{5} x_{r}^{9}+x_{m}^{5} x_{n}^{7} x_{p}^{3} x_{q}^{9} x_{r}^{5} \\
& +x_{m}^{5} x_{n}^{7} x_{p}^{5} x_{q}^{3} x_{r}^{9}+x_{m}^{5} x_{n}^{7} x_{p}^{9} x_{q}^{3} x_{r}^{5}+x_{m}^{5} x_{n}^{9} x_{p}^{3} x_{q}^{3} x_{r}^{9}+x_{m}^{5} x_{n}^{11} x_{p}^{3} x_{q}^{5} x_{r}^{5}, \\
\sum \bar{C}_{1}= & x_{m}^{3} x_{n}^{7} x_{p}^{3} x_{q}^{5} x_{r}^{9}+x_{m}^{3} x_{n}^{7} x_{p}^{3} x_{q}^{9} x_{r}^{5}+x_{m}^{3} x_{n}^{7} x_{p}^{5} x_{q}^{3} x_{r}^{9}+x_{m}^{3} x_{n}^{7} x_{p}^{9} x_{q}^{3} x_{r}^{5}+x_{m}^{3} x_{n}^{9} x_{p}^{3} x_{q}^{3} x_{r}^{9} \\
& +x_{m}^{3} x_{n}^{9} x_{p}^{5} x_{q}^{5} x_{r}^{5}+x_{m}^{3} x_{n}^{11} x_{p}^{3} x_{q}^{5} x_{r}^{5}+x_{m}^{3} x_{n}^{11} x_{p}^{5} x_{q}^{3} x_{r}^{5},
\end{aligned}
$$

Since $x_{m}^{3} x_{n}^{13} x_{p}^{3} x_{q}^{6} x_{r}^{6}<f$, the monomial $f$ is strictly inadmissible. Next, by a direct computation, we have

$$
\begin{aligned}
g= & \sum \mathcal{L}+S q^{1}\left(\sum \bar{A}_{2}\right)+S q^{2}\left(\sum \bar{B}_{2}\right)+S q^{4}\left(\sum \bar{C}_{2}\right) \text { modulo }\left(\mathscr{P}_{5}^{-}\left(\omega^{* * *}\right)\right), \text { where } \\
\sum \mathcal{L}= & x_{m}^{3} x_{n}^{3} x_{p}^{14} x_{q}^{5} x_{r}^{6}+x_{m}^{3} x_{n}^{5} x_{p}^{7} x_{q}^{6} x_{r}^{10}+x_{m}^{3} x_{n}^{5} x_{p}^{11} x_{q}^{6} x_{r}^{6}, \\
\sum \bar{A}_{2}= & x_{m}^{3} x_{n}^{3} x_{p}^{13} x_{q}^{5} x_{r}^{6}+x_{m}^{3} x_{n}^{3} x_{p}^{14} x_{q}^{5} x_{r}^{5}+x_{m}^{3} x_{n}^{5} x_{p}^{11} x_{q}^{5} x_{r}^{6}+x_{m}^{3} x_{n}^{5} x_{p}^{13} x_{q}^{3} x_{r}^{6}+x_{m}^{3} x_{n}^{6} x_{p}^{7} x_{q}^{9} x_{r}^{5} \\
& +x_{m}^{3} x_{n}^{6} x_{p}^{11} x_{q}^{5} x_{r}^{5}+x_{m}^{3} x_{n}^{10} x_{p}^{7} x_{q}^{5} x_{r}^{5}+x_{m}^{6} x_{n}^{5} x_{p}^{11} x_{q}^{3} x_{r}^{5}, \\
\sum \bar{B}_{2}= & x_{m}^{3} x_{n}^{5} x_{p}^{11} x_{q}^{5} x_{r}^{5}+x_{m}^{3} x_{n}^{5} x_{p}^{13} x_{q}^{3} x_{r}^{5}+x_{m}^{3} x_{n}^{6} x_{p}^{11} x_{q}^{3} x_{r}^{6}+x_{m}^{5} x_{n}^{3} x_{p}^{7} x_{q}^{5} x_{r}^{9}+x_{m}^{5} x_{n}^{3} x_{p}^{7} x_{q}^{9} x_{r}^{5} \\
& +x_{m}^{5} x_{n}^{3} x_{p}^{9} x_{q}^{3} x_{r}^{9}+x_{m}^{5} x_{n}^{3} x_{p}^{11} x_{q}^{5} x_{r}^{5}+x_{m}^{5} x_{n}^{5} x_{p}^{7} x_{q}^{3} x_{r}^{9}+x_{m}^{5} x_{n}^{9} x_{p}^{7} x_{q}^{3} x_{r}^{5}, \\
\sum \bar{C}_{2}= & x_{m}^{3} x_{n}^{3} x_{p}^{7} x_{q}^{5} x_{r}^{9}+x_{m}^{3} x_{n}^{3} x_{p}^{7} x_{q}^{9} x_{r}^{5}+x_{m}^{3} x_{n}^{3} x_{p}^{9} x_{q}^{3} x_{r}^{9}+x_{m}^{3} x_{n}^{3} x_{p}^{11} x_{q}^{5} x_{r}^{5}+x_{m}^{3} x_{n}^{5} x_{p}^{7} x_{q}^{3} x_{r}^{9} \\
& +x_{m}^{3} x_{n}^{5} x_{p}^{7} x_{q}^{6} x_{r}^{6}+x_{m}^{3} x_{n}^{5} x_{p}^{9} x_{q}^{5} x_{r}^{5}+x_{m}^{3} x_{n}^{5} x_{p}^{11} x_{q}^{3} x_{r}^{5}+x_{m}^{3} x_{n}^{9} x_{p}^{7} x_{q}^{3} x_{r}^{5} .
\end{aligned}
$$

These relations show that $g$ is strictly inadmissible. The lemmas follows.

The proof of the following lemmas is analogous to the proofs of Lemmas 3.3.16, 3.3.17, 3.3.28 and 3.3.29.

Lemma 3.3.30. If $(p, q, r)$ is a permutation of $(3,4,5)$, then the following monomials are strictly inadmissible:

$$
\begin{gathered}
u_{1}=x_{1} x_{2}^{6} x_{p}^{6} x_{q}^{3} x_{r}^{15}, u_{2}=x_{1}^{3} x_{2}^{6} x_{p}^{6} x_{q} x_{r}^{15}, u_{3}=x_{1}^{16} x_{2}^{6} x_{p}^{6} x_{q} x_{r}^{3}, u_{4}=x_{1} x_{2}^{6} x_{p}^{3} x_{q}^{7} x_{r}^{14}, \\
u_{5}=x_{1} x_{2}^{14} x_{p}^{3} x_{q}^{6} x_{r}^{7}, u_{6}=x_{1}^{3} x_{2}^{6} x_{p} x_{q}^{7} x_{r}^{14}, u_{7}=x_{1}^{3} x_{2}^{14} x_{p} x_{q}^{6} x_{r}^{7}, u_{8}=x_{1}^{7} x_{2}^{6} x_{p} x_{q}^{3} x_{r}^{14}, \\
u_{9}=x_{1}^{7} x_{2}^{14} x_{p} x_{q}^{3} x_{r}^{6}, u_{10}=x_{1} x_{2}^{6} x_{p}^{6} x_{q}^{7} x_{r}^{11}, u_{11}=x_{1}^{7} x_{2}^{6} x_{p} x_{q}^{6} x_{r}^{11}, u_{12}=x_{1}^{3} x_{2}^{2} x_{p}^{5} x_{q}^{6} x_{r}^{15}, \\
u_{13}=x_{1}^{3} x_{2}^{6} x_{p}^{2} x_{q}^{5} x_{r}^{15}, u_{14}=x_{1}^{15} x_{2}^{2} x_{p}^{3} x_{q}^{5} x_{r}^{6}, u_{15}=x_{1}^{15} x_{2}^{6} x_{p}^{2} x_{q}^{3} x_{r}^{5}, u_{16}=x_{1}^{3} x_{2}^{2} x_{p}^{5} x_{q}^{7} x_{r}^{14}, \\
u_{17}=x_{1}^{3} x_{2}^{14} x_{p}^{2} x_{q}^{5} x_{r}^{7}, u_{18}=x_{1}^{7} x_{2}^{2} x_{p}^{14} x_{q}^{3} x_{r}^{5}, u_{19}=x_{1}^{7} x_{2}^{14} x_{p}^{2} x_{q}^{3} x_{r}^{5} .
\end{gathered}
$$

Lemma 3.3.31. The following monomials are strictly inadmissible:

I) $u_{20}=x_{1} x_{2}^{15} x_{3}^{6} x_{q}^{3} x_{r}^{6}, u_{21}=x_{1}^{3} x_{2}^{15} x_{3}^{6} x_{q} x_{r}^{6}, u_{22}=x_{1}^{15} x_{2} x_{3}^{6} x_{q}^{3} x_{r}^{6}, u_{23}=x_{1}^{15} x_{2}^{3} x_{3}^{6} x_{q} x_{r}^{6}$, $u_{24}=x_{1}^{7} x_{2} x_{3}^{6} x_{q}^{3} x_{r}^{14}, u_{25}=x_{1}^{7} x_{2} x_{3}^{14} x_{q}^{3} x_{r}^{6}, u_{26}=x_{1}^{7} x_{2}^{3} x_{3}^{6} x_{q} x_{r}^{14}, u_{27}=x_{1}^{7} x_{2}^{3} x_{3}^{14} x_{q} x_{r}^{6}$, $u_{28}=x_{1} x_{2}^{3} x_{3}^{14} x_{q}^{6} x_{r}^{7}, u_{29}=x_{1} x_{2}^{7} x_{3}^{6} x_{q}^{3} x_{r}^{14}, u_{30}=x_{1} x_{2}^{7} x_{3}^{14} x_{q}^{3} x_{r}^{6}, u_{31}=x_{1}^{3} x_{2} x_{3}^{14} x_{q}^{6} x_{r}^{7}$, $u_{32}=x_{1}^{3} x_{2}^{7} x_{3}^{6} x_{q} x_{r}^{14}, u_{33}=x_{1}^{3} x_{2}^{7} x_{3}^{14} x_{q} x_{r}^{6}, u_{34}=x_{1} x_{2}^{7} x_{3}^{6} x_{q}^{6} x_{r}^{11}, u_{35}=x_{1}^{7} x_{2} x_{3}^{6} x_{q}^{6} x_{r}^{11}$, $u_{36}=x_{1}^{7} x_{2}^{11} x_{3}^{6} x_{q} x_{r}^{6}, u_{37}=x_{1}^{3} x_{2}^{15} x_{3}^{2} x_{q}^{5} x_{r}^{6}, u_{38}=x_{1}^{3} x_{2}^{15} x_{3}^{6} x_{q}^{2} x_{r}^{5}, u_{39}=x_{1}^{15} x_{2}^{3} x_{3}^{2} x_{q}^{5} x_{r}^{6}$, $u_{40}=x_{1}^{15} x_{2}^{3} x_{3}^{6} x_{q}^{2} x_{r}^{5}, u_{41}=x_{1}^{3} x_{2}^{5} x_{3}^{14} x_{q}^{2} x_{r}^{7}, u_{42}=x_{1}^{3} x_{2}^{5} x_{3}^{14} x_{q}^{2} x_{r}^{7}, u_{43}=x_{1}^{3} x_{2}^{7} x_{3}^{2} x_{q}^{5} x_{r}^{14}$, $u_{44}=x_{1}^{3} x_{2}^{7} x_{3}^{14} x_{q}^{2} x_{r}^{5}, u_{45}=x_{1}^{7} x_{2}^{3} x_{3}^{2} x_{q}^{5} x_{r}^{14}, u_{46}=x_{1}^{7} x_{2}^{3} x_{3}^{14} x_{q}^{2} x_{r}^{5}$, where $q, r=4,5$, $q \neq r$.

II) $u_{47}=x_{1} x_{2}^{3} x_{3}^{6} x_{4}^{14} x_{5}^{7}, u_{48}=x_{1}^{3} x_{2} x_{3}^{6} x_{4}^{14} x_{5}^{7}, u_{49}=x_{1}^{3} x_{2}^{5} x_{3}^{2} x_{4}^{14} x_{5}^{7}$.

Note that $\omega\left(u_{t}\right)=\omega^{* * *}$ for $t=1,2, \ldots, 49$. Next we consider the following lemma.

Lemma 3.3.32. The following monomials are strictly inadmissible:
$A_{1}=x_{1} x_{2}^{3} x_{3}^{6} x_{4}^{15} x_{5}^{22}$,
$A_{2}=x_{1} x_{2}^{3} x_{3}^{15} x_{4}^{6} x_{5}^{22}$,
$A_{3}=x_{1} x_{2}^{3} x_{3}^{15} x_{4}^{22} x_{5}^{6}$,
$A_{5}=x_{1} x_{2}^{15} x_{3}^{3} x_{4}^{22} x_{5}^{6}$,
$A_{6}=x_{1} x_{2}^{15} x_{3}^{19} x_{4}^{6} x_{5}^{6}$,
$A_{7}=x_{1}^{3} x_{2} x_{3}^{6} x_{4}^{15} x_{5}^{22}$,
$A_{9}=x_{1}^{3} x_{2}^{5} x_{3}^{6} x_{4}^{15} x_{5}^{18}$,
$A_{10}=x_{1}^{3} x_{2} x_{3}^{15} x_{4}^{6} x_{5}^{22}$
$A_{11}=x_{1}^{3} x_{2} x_{3}^{15} x_{4}^{22} x_{5}^{6}$,
$A_{13}=x_{1}^{3} x_{2}^{5} x_{3}^{15} x_{4}^{6} x_{5}^{18}$,
$A_{14}=x_{1}^{3} x_{2}^{5} x_{3}^{15} x_{4}^{18} x_{5}^{6}$,
$A_{15}=x_{1}^{3} x_{2}^{5} x_{3}^{15} x_{4}^{22} x_{5}^{2}$,
$A_{17}=x_{1}^{3} x_{2}^{13} x_{3}^{3} x_{4}^{22} x_{5}^{6}$,
$A_{21}=x_{1}^{3} x_{2}^{15} x_{3}^{5} x_{4}^{2} x_{5}^{22}$,
$A_{18}=x_{1}^{3} x_{2}^{13} x_{3}^{19} x_{4}^{6} x_{5}^{6}$,
$A_{19}=x_{1}^{3} x_{2}^{15} x_{3} x_{4}^{6} x_{5}^{22}$,
$A_{22}=x_{1}^{3} x_{2}^{15} x_{3}^{5} x_{4}^{6} x_{5}^{18}$,
$A_{25}=x_{1}^{3} x_{2}^{15} x_{3}^{17} x_{4}^{6} x_{5}^{6}$,
$A_{26}=x_{1}^{3} x_{2}^{15} x_{3}^{21} x_{4}^{2} x_{5}^{6}$,
$A_{23}=x_{1}^{3} x_{2}^{15} x_{3}^{5} x_{4}^{18} x_{5}^{6}$,
$A_{27}=x_{1}^{3} x_{2}^{15} x_{3}^{21} x_{4}^{6} x_{5}^{2}$,
$A_{29}=x_{1}^{7} x_{2}^{9} x_{3}^{3} x_{4}^{22} x_{5}^{6}$,
$A_{30}=x_{1}^{7} x_{2}^{9} x_{3}^{19} x_{4}^{6} x_{5}^{6}$,
$A_{31}=x_{1}^{7} x_{2}^{11} x_{3}^{17} x_{4}^{6} x_{5}^{6}$,
$A_{33}=x_{1}^{15} x_{2} x_{3}^{3} x_{4}^{6} x_{5}^{22}$,
$A_{34}=x_{1}^{15} x_{2} x_{3}^{3} x_{4}^{22} x_{5}^{6}$
$A_{35}=x_{1}^{15} x_{2} x_{3}^{19} x_{4}^{6} x_{5}^{6}$,
$A_{37}=x_{1}^{15} x_{2}^{3} x_{3} x_{4}^{22} x_{5}^{6}$,
$A_{38}=x_{1}^{15} x_{2}^{3} x_{3}^{5} x_{4}^{2} x_{5}^{22}$,
$A_{39}=x_{1}^{15} x_{2}^{3} x_{3}^{5} x_{4}^{6} x_{5}^{18}$,
$A_{41}=x_{1}^{15} x_{2}^{3} x_{3}^{5} x_{4}^{22} x_{5}^{2}$,
$A_{42}=x_{1}^{15} x_{2}^{3} x_{3}^{17} x_{4}^{6} x_{5}^{6}$,
$A_{43}=x_{1}^{15} x_{2}^{3} x_{3}^{21} x_{4}^{2} x_{5}^{6}$,
$A_{45}=x_{1}^{15} x_{2}^{17} x_{3}^{3} x_{4}^{6} x_{5}^{6}$,
$A_{49}=x_{1}^{3} x_{2}^{5} x_{3}^{6} x_{4}^{14} x_{5}^{19}$,
$A_{46}=x_{1}^{15} x_{2}^{19} x_{3} x_{4}^{6} x_{5}^{6}$,
$A_{50}=x_{1}^{3} x_{2}^{5} x_{3}^{6} x_{4}^{6} x_{5}^{27}$,

$$
\begin{aligned}
& A_{4}=x_{1} x_{2}^{15} x_{3}^{3} x_{4}^{6} x_{5}^{22}, \\
& A_{8}=x_{1}^{3} x_{2}^{5} x_{3}^{2} x_{4}^{15} x_{5}^{22}, \\
& A_{12}=x_{1}^{3} x_{2}^{5} x_{3}^{15} x_{4}^{2} x_{5}^{22}, \\
& A_{16}=x_{1}^{3} x_{2}^{13} x_{3}^{3} x_{4}^{6} x_{5}^{22}, \\
& A_{20}=x_{1}^{3} x_{2}^{15} x_{3} x_{4}^{22} x_{5}^{6}, \\
& A_{24}=x_{1}^{3} x_{2}^{15} x_{3}^{5} x_{4}^{22} x_{5}^{2}, \\
& A_{28}=x_{1}^{7} x_{2}^{9} x_{3}^{3} x_{4}^{6} x_{5}^{22}, \\
& A_{32}=x_{1}^{7} x_{2}^{25} x_{3}^{3} x_{4}^{6} x_{5}^{6}, \\
& A_{36}=x_{1}^{15} x_{2}^{3} x_{3} x_{4}^{6} x_{5}^{22}, \\
& A_{40}=x_{1}^{15} x_{2}^{3} x_{3}^{5} x_{4}^{18} x_{5}^{6}, \\
& A_{44}=x_{1}^{15} x_{2}^{3} x_{3}^{21} x_{4}^{6} x_{5}^{2}, \\
& A_{48}=x_{1}^{15} x_{2}^{19} x_{3}^{5} x_{4}^{6} x_{5}^{2},
\end{aligned}
$$$$
A_{47}=x_{1}^{15} x_{2}^{19} x_{3}^{5} x_{4}^{2} x_{5}^{6} \text {, }
$$

Proof. We have $\omega\left(A_{i}\right)=\bar{\omega}_{(4)}, 1 \leq i \leq 51$. We prove the lemma for the monomials $A_{23}=$ $x_{1}^{3} x_{2}^{15} x_{3}^{5} x_{4}^{18} x_{5}^{6}$ and $A_{29}=x_{1}^{7} x_{2}^{9} x_{3}^{3} x_{4}^{22} x_{5}^{6}$. The others can be proved by using a similar technique as 
in Lemmas 3.3.20 and 3.3.21. Direct computing from Cartan's formula, we get

$$
\begin{aligned}
A_{23}= & \sum_{1 \leq i \leq 3} b_{i}+S q^{1}\left(\sum f\right)+S q^{2}\left(\sum g\right)+S q^{4}\left(\sum h\right)+S q^{8}\left(\sum p\right) \text { modulo }\left(\mathscr{P}_{5}^{-}\left(\bar{\omega}_{(4)}\right),\right. \text { where } \\
b_{1}= & x_{1}^{3} x_{2}^{11} x_{3}^{5} x_{4}^{22} x_{5}^{6}, b_{2}=x_{1}^{3} x_{2}^{13} x_{3}^{3} x_{4}^{22} x_{5}^{6}, b_{3}=x_{1}^{3} x_{2}^{13} x_{3}^{6} x_{4}^{19} x_{5}^{6}, \\
\sum f= & x_{1}^{3} x_{2}^{7} x_{3}^{6} x_{4}^{21} x_{5}^{9}+x_{1}^{3} x_{2}^{7} x_{3}^{6} x_{4}^{25} x_{5}^{5}+x_{1}^{3} x_{2}^{11} x_{3}^{6} x_{4}^{21} x_{5}^{5}+x_{1}^{3} x_{2}^{14} x_{3}^{3} x_{4}^{21} x_{5}^{5}+x_{1}^{3} x_{2}^{15} x_{3}^{5} x_{4}^{14} x_{5}^{9} \\
& +x_{1}^{3} x_{2}^{15} x_{3}^{5} x_{4}^{17} x_{5}^{6}+x_{1}^{3} x_{2}^{15} x_{3}^{9} x_{4}^{14} x_{5}^{5}+x_{1}^{3} x_{2}^{19} x_{3}^{5} x_{4}^{14} x_{5}^{5}+x_{1}^{3} x_{2}^{22} x_{3}^{5} x_{4}^{11} x_{5}^{5}, \\
\sum g= & x_{1}^{5} x_{2}^{7} x_{3}^{3} x_{4}^{21} x_{5}^{9}+x_{1}^{5} x_{2}^{7} x_{3}^{3} x_{4}^{25} x_{5}^{5}+x_{1}^{5} x_{2}^{9} x_{3}^{9} x_{4}^{19} x_{5}^{3}+x_{1}^{5} x_{2}^{11} x_{3}^{3} x_{4}^{17} x_{5}^{9}+x_{1}^{5} x_{2}^{15} x_{3}^{5} x_{4}^{11} x_{5}^{9} \\
& +x_{1}^{5} x_{2}^{15} x_{3}^{9} x_{4}^{11} x_{5}^{5}+x_{1}^{5} x_{2}^{17} x_{3}^{3} x_{4}^{17} x_{5}^{3}+x_{1}^{5} x_{2}^{17} x_{3}^{9} x_{4}^{11} x_{5}^{3}+x_{1}^{5} x_{2}^{19} x_{3}^{5} x_{4}^{11} x_{5}^{5}, \\
\sum & x_{1}^{3} x_{2}^{7} x_{3}^{3} x_{4}^{21} x_{5}^{9}+x_{1}^{3} x_{2}^{7} x_{3}^{3} x_{4}^{25} x_{5}^{5}+x_{1}^{3} x_{2}^{9} x_{3}^{5} x_{4}^{21} x_{5}^{5}+x_{1}^{3} x_{2}^{9} x_{3}^{9} x_{4}^{19} x_{5}^{3}+x_{1}^{3} x_{2}^{11} x_{3}^{3} x_{4}^{17} x_{5}^{9} \\
& +x_{1}^{3} x_{2}^{11} x_{3}^{3} x_{4}^{21} x_{5}^{5}+x_{1}^{3} x_{2}^{13} x_{3}^{9} x_{4}^{13} x_{5}^{5}+x_{1}^{3} x_{2}^{15} x_{3}^{5} x_{4}^{11} x_{5}^{9}+x_{1}^{3} x_{2}^{15} x_{3}^{6} x_{4}^{13} x_{5}^{6}+x_{1}^{3} x_{2}^{15} x_{3}^{9} x_{4}^{11} x_{5}^{5} \\
& +x_{1}^{3} x_{2}^{17} x_{3}^{3} x_{4}^{17} x_{5}^{3}+x_{1}^{3} x_{2}^{17} x_{3}^{9} x_{4}^{11} x_{5}^{3}+x_{1}^{3} x_{2}^{19} x_{3}^{5} x_{4}^{11} x_{5}^{5}, \\
\sum & x_{1}^{3} x_{2}^{13} x_{3}^{5} x_{4}^{13} x_{5}^{5}+x_{1}^{3} x_{2}^{13} x_{3}^{6} x_{4}^{11} x_{5}^{6} .
\end{aligned}
$$

Since $b_{i}<A_{23}, 1 \leq i \leq 3, A_{23}$ is strictly inadmissible. By a similar computation, we obtain

$$
\begin{aligned}
& A_{29}=\sum Z+S q^{1}\left(\sum \bar{f}\right)+S q^{2}\left(\sum \bar{g}\right)+S q^{4}\left(\sum \bar{h}\right)+S q^{8}\left(x_{1}^{7} x_{2}^{5} x_{3}^{6} x_{4}^{15} x_{5}^{6}\right) \text { modulo }\left(\mathscr{P}_{5}^{-}\left(\bar{\omega}_{(4)}\right)\right. \text {, where, } \\
& \sum Z=x_{1}^{5} x_{2}^{3} x_{3}^{6} x_{4}^{23} x_{5}^{10}+x_{1}^{5} x_{2}^{3} x_{3}^{6} x_{4}^{27} x_{5}^{6}+x_{1}^{5} x_{2}^{3} x_{3}^{10} x_{4}^{23} x_{5}^{6}+x_{1}^{5} x_{2}^{7} x_{3}^{3} x_{4}^{22} x_{5}^{10}+x_{1}^{5} x_{2}^{7} x_{3}^{3} x_{4}^{26} x_{5}^{6} \\
& +x_{1}^{5} x_{2}^{7} x_{3}^{6} x_{4}^{19} x_{5}^{10}+x_{1}^{5} x_{2}^{7} x_{3}^{10} x_{4}^{19} x_{5}^{6}+x_{1}^{5} x_{2}^{11} x_{3}^{3} x_{4}^{22} x_{5}^{6}+x_{1}^{5} x_{2}^{11} x_{3}^{6} x_{4}^{19} x_{5}^{6}+x_{1}^{7} x_{2}^{3} x_{3}^{5} x_{4}^{22} x_{5}^{10} \\
& +x_{1}^{7} x_{2}^{3} x_{3}^{6} x_{4}^{23} x_{5}^{8}+x_{1}^{7} x_{2}^{3} x_{3}^{6} x_{4}^{25} x_{5}^{6}+x_{1}^{7} x_{2}^{3} x_{3}^{8} x_{4}^{23} x_{5}^{6}+x_{1}^{7} x_{2}^{3} x_{3}^{9} x_{4}^{22} x_{5}^{6}+x_{1}^{7} x_{2}^{7} x_{3}^{3} x_{4}^{22} x_{5}^{8} \\
& +x_{1}^{7} x_{2}^{7} x_{3}^{3} x_{4}^{24} x_{5}^{6} \\
& \sum \bar{f}=x_{1}^{7} x_{2}^{3} x_{3}^{5} x_{4}^{19} x_{5}^{12}+x_{1}^{7} x_{2}^{3} x_{3}^{5} x_{4}^{21} x_{5}^{10}+x_{1}^{7} x_{2}^{3} x_{3}^{9} x_{4}^{21} x_{5}^{6}+x_{1}^{7} x_{2}^{3} x_{3}^{12} x_{4}^{19} x_{5}^{5}+x_{1}^{7} x_{2}^{6} x_{3}^{9} x_{4}^{15} x_{5}^{9} \\
& +x_{1}^{7} x_{2}^{7} x_{3}^{5} x_{4}^{19} x_{5}^{8}+x_{1}^{7} x_{2}^{7} x_{3}^{5} x_{4}^{21} x_{5}^{6}+x_{1}^{7} x_{2}^{7} x_{3}^{8} x_{4}^{19} x_{5}^{5}+x_{1}^{7} x_{2}^{10} x_{3}^{5} x_{4}^{15} x_{5}^{9} \\
& +x_{1}^{7} x_{2}^{10} x_{3}^{9} x_{4}^{15} x_{5}^{5}, \\
& \sum \bar{g}=x_{1}^{7} x_{2}^{3} x_{3}^{6} x_{4}^{19} x_{5}^{10}+x_{1}^{7} x_{2}^{3} x_{3}^{6} x_{4}^{23} x_{5}^{6}+x_{1}^{7} x_{2}^{3} x_{3}^{9} x_{4}^{17} x_{5}^{9}+x_{1}^{7} x_{2}^{3} x_{3}^{10} x_{4}^{19} x_{5}^{6}+x_{1}^{7} x_{2}^{5} x_{3}^{9} x_{4}^{15} x_{5}^{9} \\
& +x_{1}^{7} x_{2}^{7} x_{3}^{3} x_{4}^{22} x_{5}^{6}+x_{1}^{7} x_{2}^{7} x_{3}^{6} x_{4}^{19} x_{5}^{6}+x_{1}^{7} x_{2}^{9} x_{3}^{3} x_{4}^{17} x_{5}^{9}+x_{1}^{7} x_{2}^{9} x_{3}^{5} x_{4}^{15} x_{5}^{9}+x_{1}^{7} x_{2}^{9} x_{3}^{9} x_{4}^{15} x_{5}^{5} \\
& +x_{1}^{7} x_{2}^{9} x_{3}^{9} x_{4}^{17} x_{5}^{3}+x_{1}^{9} x_{2}^{3} x_{3}^{9} x_{4}^{15} x_{5}^{9}+x_{1}^{9} x_{2}^{9} x_{3}^{3} x_{4}^{15} x_{5}^{9}+x_{1}^{9} x_{2}^{9} x_{3}^{9} x_{4}^{15} x_{5}^{3} \text {, } \\
& \sum \bar{h}=x_{1}^{5} x_{2}^{3} x_{3}^{6} x_{4}^{23} x_{5}^{6}+x_{1}^{5} x_{2}^{7} x_{3}^{3} x_{4}^{22} x_{5}^{6}+x_{1}^{5} x_{2}^{7} x_{3}^{6} x_{4}^{19} x_{5}^{6} \text {. }
\end{aligned}
$$

The above equalities imply that $A_{29}$ is strictly inadmissible. The lemma is proved.

Proof of Lemma 3.3.26. Let $b$ be an admissible monomial in $\left(\mathscr{P}_{5}^{+}\right)_{47}$ such that $\omega(b)=\bar{\omega}_{(4)}$. Then $\omega_{1}(b)=3$ and $b=X_{(\{t, k\}, 5)} Y^{2}$ with $1 \leq t<k \leq 5$ and $Y$ a monomial of degree 22 in $\mathscr{P}_{5}$. Since $b$ is admissible, according to Theorem 2.2.1, $Y \in \mathscr{B}_{5}\left(\omega_{(4)}\right)$.

Using Proposition 3.3.14(III) and a simple computation shows that if $Z \in \mathscr{B}_{5}\left(\omega_{(4)}\right), 1 \leq t<$ $k \leq 5$, and $X_{(\{t, k\}, 5)} Z^{2} \neq y_{47, j}, \forall j, 371 \leq j \leq 479$, then there is a monomial $u$ which is given in one of Lemmas 3.3.27 - 3.3.32 such that $X_{(\{t, k\}, 5)} Z^{2}=u g^{2^{s}}$ with a monomial $g \in \mathscr{P}_{5}$, and $s=\max \left\{\ell \in \mathbb{Z}: \omega_{\ell}(u)>0\right\}$. Then, by Theorem 2.2.1, $X_{(\{t, k\}, 5)} Z^{2}$ is inadmissible. Finally, we see that $b=X_{(\{t, k\}, 5)} Y^{2}$ is admissible with $Y \in \mathscr{B}_{5}\left(\omega_{(4)}\right)$; hence $b=y_{47, j}$ for some $j \in\{371, \ldots, 479\}$. This implies $\mathscr{B}_{5}^{+}\left(\bar{\omega}_{(4)}\right) \subseteq\left\{y_{47, j}: 371 \leq j \leq 479\right\}$.

Proposition 3.3.33. $Q \mathscr{P}_{5}^{+}\left(\bar{\omega}_{(4)}\right)$ is the $\mathbb{Z} / 2$-vector space of dimension 109 with a basis consisting of all the classes represented by the monomials $Y_{47, j}, 371 \leq j \leq 479$.

Proof. First, we show that the set $\left[V:=\left\{Y_{47, j}: 371 \leq j \leq 479\right\}\right]_{\bar{\omega}_{(4)}}$ is linearly independent in the space $Q \mathscr{P}_{5}^{+}\left(\bar{\omega}_{(4)}\right)$. Indeed, suppose there is a linear relation $\mathcal{S}=\sum_{371 \leq j \leq 479} \gamma_{j} y_{j} \equiv_{\bar{\omega}_{(4)}} 0$ with $\gamma_{j} \in \mathbb{Z} / 2$ and $y_{47, j} \in V$. By using Theorem 3.1.3 and Proposition 3.2.1, we determine explicitly $\pi_{(k ; \mathscr{K})}(\mathcal{S})$ in terms of the admissible monomials in $\left(\mathscr{P}_{4}^{+}\right)_{47}$. From the relations $\pi_{(k ; \mathscr{K})}(\mathcal{S}) \equiv_{\bar{\omega}_{(4)}} 0$ with $\ell(\mathscr{K})>0$, one gets $\gamma_{j}=0$ for $j=371,372, \ldots, 479$.

Now, by Lemma 3.3.26, to prove $[V]_{\bar{\omega}_{(4)}}$ is a basis of $Q \mathscr{P}_{5}^{+}\left(\bar{\omega}_{(4)}\right)$ we need to show that $\left[y_{47, j}\right]_{\bar{\omega}_{(4)}} \neq$ [0] for all $y_{47, j} \in V$. By a similar argument as given in the proof of Propositions 3.3.1 and 3.3.10, we can prove that the set $\left[\mathscr{B}_{5}^{+}\left(\bar{\omega}_{(1)}\right) \cup V\right]$ is linearly independent in $\left(Q \mathscr{P}_{5}^{+}\right)_{47}$. This fact shows that $\left[y_{47, j}\right]_{\bar{\omega}_{(4)}} \neq[0]$ for all $y_{47, j}$. The proposition is proved.

Proposition 3.3.34. There exist exactly 15 admissible monomials in $\left(\mathscr{P}_{5}^{+}\right)_{47}$ such that their weight vectors are $\bar{\omega}_{(5)}$. Consequently $\operatorname{dim}\left(Q P_{5}^{+}\left(\bar{\omega}_{(5)}\right)\right)=15$.

We prove the proposition by showing that

$$
\mathscr{B}_{5}^{+}\left(\bar{\omega}_{(5)}\right)=\left\{y_{47, m}: 480 \leq m \leq 494\right\},
$$

where the monomials $y_{47, m}: 480 \leq m \leq 494$, are listed in Sect.6.7 of the online version [44]. We need some lemmas for the proof of this proposition. The Lemmas 3.3.35 and 3.3.36 below can be proved by using a result in [69]. 
Lemma 3.3.35. The following monomials are strictly inadmissible:

a) $x_{u} x_{v}^{2} x_{m}^{6} x_{n}^{7} x_{p}^{7}, x_{u}^{7} x_{v}^{2} x_{m}^{2} x_{n}^{5} x_{p}^{7}, x_{u}^{3} x_{v}^{2} x_{m}^{4} x_{n}^{7} x_{p}^{7}, x_{u}^{3} x_{v}^{4} x_{m}^{6} x_{n}^{3} x_{p}^{7}, x_{u}^{3} x_{v}^{6} x_{m}^{6} x_{n}^{3} x_{p}^{5}$, where $(u, v, m, n, p)$ is a permutation of $(1,2,3,4,5)$;

b) $x_{1} x_{2}^{6} x_{q}^{6} x_{r}^{3} x_{t}^{7}, x_{1}^{3} x_{2}^{6} x_{q}^{6} x_{r} x_{t}^{7}, x_{1}^{7} x_{2}^{6} x_{q}^{6} x_{r} x_{t}^{3}, x_{1}^{3} x_{2}^{2} x_{q}^{6} x_{r}^{5} x_{t}^{7}, x_{1}^{3} x_{2}^{6} x_{q}^{2} x_{r}^{5} x_{t}^{7}, x_{1}^{7} x_{2}^{2} x_{q}^{6} x_{r}^{3} x_{t}^{5}$, $x_{1}^{7} x_{2}^{6} x_{q}^{2} x_{r}^{3} x_{t}^{5}$, where $(q, r, t)$ is a permutation of $(3,4,5)$;

c) $x_{1} x_{2}^{7} x_{3}^{6} x_{4}^{6} x_{5}^{3}, \quad x_{1} x_{2}^{7} x_{3}^{6} x_{4}^{3} x_{5}^{6}, \quad x_{1}^{3} x_{2}^{7} x_{3}^{6} x_{4}^{6} x_{5}, \quad x_{1}^{3} x_{2}^{7} x_{3}^{6} x_{4} x_{5}^{6}$, $x_{1}^{7} x_{2} x_{3}^{6} x_{4}^{6} x_{5}^{3}, \quad x_{1}^{7} x_{2}^{3} x_{3}^{6} x_{4}^{6} x_{5}, \quad x_{1}^{7} x_{2} x_{3}^{6} x_{4}^{3} x_{5}^{6}, \quad x_{1}^{7} x_{2}^{3} x_{3}^{6} x_{4} x_{5}^{6}$, $x_{1}^{3} x_{2}^{7} x_{3}^{2} x_{4}^{6} x_{5}^{5}, \quad x_{1}^{3} x_{2}^{7} x_{3}^{6} x_{4}^{2} x_{5}^{5}, \quad x_{1}^{3} x_{2}^{7} x_{3}^{2} x_{4}^{5} x_{5}^{6}, \quad x_{1}^{3} x_{2}^{7} x_{3}^{6} x_{4}^{5} x_{5}^{2}$, $x_{1}^{7} x_{2}^{3} x_{3}^{2} x_{4}^{6} x_{5}^{5}, \quad x_{1}^{7} x_{2}^{3} x_{3}^{6} x_{4}^{2} x_{5}^{5}, \quad x_{1}^{7} x_{2}^{3} x_{3}^{2} x_{4}^{5} x_{5}^{6}, \quad x_{1}^{7} x_{2}^{3} x_{3}^{6} x_{4}^{5} x_{5}^{2}$.

Lemma 3.3.36. Let $Z$ be the set of the following monomials:

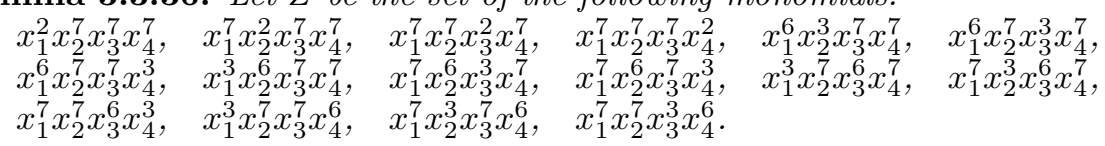

Then, the monomials $\rho_{(k, 5)}(Z), 1 \leq k \leq 5$, are strictly inadmissible.

Lemma 3.3.37. The following monomials are strictly inadmissible:

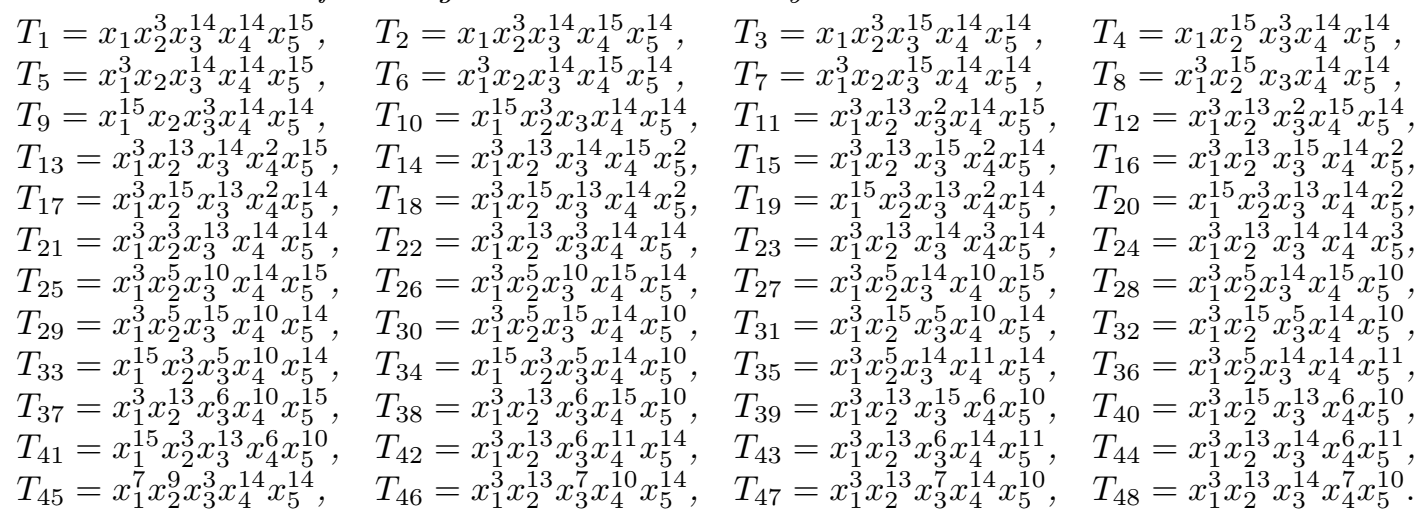

Proof. It is easy to see that $\omega\left(T_{s}\right)=\bar{\omega}_{(5)}$ for $s=1,2, \ldots, 48$. We prove the lemma for the monomials $T_{35}$ and $T_{42}$. The others can be proved by using a similar technique as in Lemmas 3.3.20, 3.3.21 and 3.3.32. Computing from the Cartan formula, we get

$$
\begin{aligned}
T_{35}= & S q^{1}\left(x_{1}^{3} x_{2}^{6} x_{3}^{11} x_{4}^{13} x_{5}^{13}+x_{1}^{3} x_{2}^{6} x_{3}^{13} x_{4}^{11} x_{5}^{13}\right)+S q^{2}\left(x_{1}^{5} x_{2}^{3} x_{3}^{7} x_{4}^{13} x_{5}^{17}+x_{1}^{5} x_{2}^{3} x_{3}^{7} x_{4}^{17} x_{5}^{13}\right. \\
& +x_{1}^{5} x_{2}^{3} x_{3}^{9} x_{4}^{11} x_{5}^{17}+x_{1}^{5} x_{2}^{3} x_{3}^{9} x_{4}^{17} x_{5}^{11}+x_{1}^{5} x_{2}^{3} x_{3}^{11} x_{4}^{9} x_{5}^{17}+x_{1}^{5} x_{2}^{3} x_{3}^{11} x_{4}^{13} x_{5}^{13} \\
& \left.+x_{1}^{5} x_{2}^{3} x_{3}^{13} x_{4}^{7} x_{5}^{17}+x_{1}^{5} x_{2}^{3} x_{3}^{13} x_{4}^{11} x_{5}^{13}+x_{1}^{5} x_{2}^{3} x_{3}^{17} x_{4}^{7} x_{5}^{13}+x_{1}^{5} x_{2}^{3} x_{3}^{17} x_{4}^{9} x_{5}^{11}\right) \\
& +S q^{4}(f)+x_{1}^{3} x_{2}^{5} x_{3}^{11} x_{4}^{14} x_{5}^{14} \operatorname{modulo}\left(\mathscr{P}_{5}^{-}\left(\bar{\omega}_{(5)}\right)\right),
\end{aligned}
$$

where the polynomial $f$ is determined as follows:

$$
\begin{aligned}
f= & x_{1}^{3} x_{2}^{3} x_{3}^{7} x_{4}^{13} x_{5}^{17}+x_{1}^{3} x_{2}^{3} x_{3}^{7} x_{4}^{17} x_{5}^{13}+x_{1}^{3} x_{2}^{3} x_{3}^{9} x_{4}^{11} x_{5}^{17}+x_{1}^{3} x_{2}^{3} x_{3}^{9} x_{4}^{17} x_{5}^{11} \\
& +x_{1}^{3} x_{2}^{3} x_{3}^{11} x_{4}^{9} x_{5}^{17}+x_{1}^{3} x_{2}^{3} x_{3}^{11} x_{4}^{13} x_{5}^{13}+x_{1}^{3} x_{2}^{3} x_{3}^{13} x_{4}^{7} x_{5}^{17}+x_{1}^{3} x_{2}^{3} x_{3}^{13} x_{4}^{11} x_{5}^{13} \\
& +x_{1}^{3} x_{2}^{3} x_{3}^{17} x_{4}^{7} x_{5}^{13}+x_{1}^{3} x_{2}^{3} x_{3}^{17} x_{4}^{9} x_{5}^{11} .
\end{aligned}
$$

Obviously, $T_{35}>x_{1}^{3} x_{2}^{5} x_{3}^{11} x_{4}^{14} x_{5}^{14}$, hence $T_{35}$ is strictly inadmissible. By a similar computation, we claim that $T_{42}=\left(p_{1}+p_{2}\right) \operatorname{modulo}\left(\mathcal{A}_{2}^{+} \mathscr{P}_{5}+\mathscr{P}_{5}^{-}\left(\bar{\omega}_{(5)}\right)\right)$, where $p_{1}=x_{1}^{3} x_{2}^{7} x_{3}^{10} x_{4}^{13} x_{5}^{14}$, and $p_{2}=x_{1}^{3} x_{2}^{13} x_{3}^{3} x_{4}^{14} x_{5}^{14}$. This completes the proof.

Proof of Proposition 3.3.34. Suppose that $X$ is a admissible monomial in $\mathscr{P}_{5}^{+}$and $\omega(X)=\bar{\omega}_{(5)}$. Then $\omega_{1}(X)=3$ and $X=x_{a} x_{b} x_{c} u^{2}$ with $1 \leq a<b<c \leq 5$ and $u \in\left(\mathscr{P}_{5}^{+}\right)_{22}$. Then, by Theorem 2.2.1, $u$ is admissible and $u \in \mathscr{B}_{5}^{+}\left(\omega_{(5)}\right)$.

Let $Z \in B_{5}^{+}\left(\omega_{(5)}\right)$ such that $x_{a} x_{b} x_{c} Z^{2} \in\left(\mathscr{P}_{5}^{+}\right)_{47}$ with $1 \leq a<b<c \leq 5$. Denote by $\bar{V}$ the set of all the monomials as given in Proposition 3.3.34. By a direct computation using Proposition 3.3.14(IV), we see that if $x_{a} x_{b} x_{c} Z^{2} \notin \bar{V}$ then there is a monomial $X_{1}$ which is given in Lemmas 3.3.35, 3.3.36 and 3.3.37 such that $x_{a} x_{b} x_{c} Z^{2}=X_{1} Z_{1}^{2^{t}}$ with suitable monomial $Z_{1} \in \mathscr{P}_{5}$ and $t=\max \left\{q \in \mathbb{Z}: \omega_{q}\left(X_{1}\right)>0\right\}$. Based on Theorem 2.2.1, we deduce that $x_{a} x_{b} x_{c} Z^{2}$ is inadmissible. Combining this with the above data, one gets $X \in \bar{V}$. This means $\mathscr{B}_{5}^{+}\left(\bar{\omega}_{(5)}\right) \subseteq \bar{V}$.

Next, we show that the set $[\bar{V}]_{\bar{\omega}_{(5)}}$ is linearly independent in the space $Q \mathscr{P}_{5}^{+}\left(\bar{\omega}_{(5)}\right)$. Indeed, suppose there is a linear relation $\mathcal{S}=\sum_{480 \leq m \leq 494} \gamma_{m} y_{47, m} \equiv_{\bar{\omega}_{(5)}} 0$, where with $\gamma_{m} \in \mathbb{Z} / 2, m=$ $480, \ldots, 494$ and $y_{47, m} \in \bar{V}$. By combining Theorem 3.1.3 and Proposition 3.2.1, we explicitly calculate $\pi_{(k ; \mathscr{K})}(\mathcal{S})$ in terms of a given minimal set of $\mathcal{A}_{2}$-generators in $\mathscr{P}_{4}\left(\operatorname{modulo}\left(\mathcal{A}_{2}^{+} \mathscr{P}_{4}\right)\right)$. From the relations $\pi_{(k ; \mathscr{K})}(\mathcal{S}) \equiv_{\bar{\omega}_{(5)}} 0$ with $\ell(\mathscr{K}) \leq 2$, we get $\gamma_{m}=0$ for all $m$.

To prove $[\bar{V}]_{\bar{\omega}_{(5)}}$ is a basis of $Q \mathscr{P}_{5}^{+}\left(\bar{\omega}_{(5)}\right)$ we need to show that $\left[y_{47, m}\right]_{\bar{\omega}_{(5)}} \neq[0]$ for all $y_{47, m} \in \bar{V}$. Denote by $\bar{V}$ the set of all the monomials as given in Proposition 3.3.34. By a similar argument as 
given in the proof of Propositions 3.3.1 and 3.3.10, we can prove that the set $\left[\mathscr{B}_{5}^{+}\left(\bar{\omega}_{(1)}\right) \cup V \cup \bar{V}\right]$ is linearly independent in $\left(Q \mathscr{P}_{5}^{+}\right)_{47}$, where $V$ the set of all the admissible monomials as given in Proposition 3.3.33. This implies $\left[y_{47, m}\right]_{\bar{\omega}_{(5)}} \neq[0]$ for all $y_{47, m}$. The proposition is proved.

Now, since $\operatorname{dim}\left(Q \mathscr{P}_{5}^{0}\right)_{47}=560$ and $\operatorname{dim}\left(Q \mathscr{P}_{5}^{0}\right)_{21}=460$, by Corollaries 3.3.15, 3.3.23 and Propositions $3.3 .25,3.3 .33,3.3 .34$, we conclude that $Q \mathscr{P}_{5}$ has dimension 1894 in degree 47 . The proof of Theorem 1.1 is completed.

Final remarks. Recall that Kameko's map $\left(\widetilde{S q_{*}^{0}}\right)_{\left(5,13.2^{t}-5\right)}$ is an epimorphism of $\mathbb{Z} / 2\left(G L_{5}\right)$ modules. This implies that $\left(Q \mathscr{P}_{5}\right)_{13.2^{t}-5} \cong \operatorname{Ker}\left(\widetilde{S q_{*}^{0}}\right)_{\left(5,13.2^{t}-5\right)} \bigoplus\left(Q \mathscr{P}_{5}\right)_{13.2^{t-1}-5}$. From a result in [70], we deduce that $\mathscr{B}_{5}\left(13.2^{0}-5\right)=\bigcup_{1 \leq j \leq 3} \mathscr{B}_{5}\left(\widetilde{\omega}_{(j)}\right)$, where $\widetilde{\omega}_{(1)}=(2,1,1)$, $\widetilde{\omega}_{(2)}=(2,3)$, and $\widetilde{\omega}_{(3)}=(4,2)$. For $m, k \in \mathbb{N}$ and $1 \leq k \leq 5$, we denote

$$
\overline{\mathscr{B}}(k, 8):=\left\{x_{k}^{2^{m}-1} \rho_{(k, 5)}(x) \in\left(\mathscr{P}_{5}\right)_{8}: x \in \mathscr{B}_{4}\left(9-2^{m}\right), \alpha\left(13-2^{m}\right) \leq 4\right\} .
$$

As well known (see [28]), $\overline{\mathscr{B}}(k, 8) \subseteq \mathscr{B}_{5}(8)$ for all $k, 1 \leq k \leq 5$. We set

$$
\overline{\mathscr{B}}\left(k, \widetilde{\omega}_{(j)}\right):=\overline{\mathscr{B}}(k, 8) \cap \mathscr{P}_{5}\left(\widetilde{\omega}_{(j)}\right) \text { for } 1 \leq j \leq 3,1 \leq k \leq 5 .
$$

Then, by a simple computation, we get

$$
\begin{gathered}
\left|\bar{\Phi}\left(\mathscr{B}_{4}\left(\widetilde{\omega}_{(1)}\right)\right) \bigcup\left(\bigcup_{1 \leq k \leq 5} \overline{\mathscr{B}}\left(k, \widetilde{\omega}_{(1)}\right)\right)\right|=105,\left|\bar{\Phi}\left(\mathscr{B}_{4}\left(\widetilde{\omega}_{(2)}\right)\right) \bigcup\left(\bigcup_{1 \leq k \leq 5} \overline{\mathscr{B}}\left(k, \widetilde{\omega}_{(2)}\right)\right)\right|=24, \\
\left|\bar{\Phi}\left(\mathscr{B}_{4}\left(\widetilde{\omega}_{(3)}\right)\right) \bigcup\left(\bigcup_{1 \leq k \leq 5} \overline{\mathscr{B}}\left(k, \widetilde{\omega}_{(3)}\right)\right)\right|=45 .
\end{gathered}
$$

Furthermore,

$$
\mathscr{B}_{5}\left(\widetilde{\omega}_{(j)}\right)=\bar{\Phi}\left(\mathscr{B}_{4}\left(\widetilde{\omega}_{(j)}\right)\right) \bigcup\left(\bigcup_{1 \leq k \leq 5} \overline{\mathscr{B}}\left(k, \widetilde{\omega}_{(j)}\right)\right), j=1,2,3 .
$$

Note that $\sum_{1 \leq j \leq 3}\left|\mathscr{B}_{5}\left(\widetilde{\omega}_{(j)}\right)\right|=\operatorname{dim}\left(Q \mathscr{P}_{5}\right)_{13.2^{0}-5}=105+24+45=174$.

Now, according to Lemma 3.3.2, $\operatorname{Ker}\left(\widetilde{S q_{*}^{0}}\right)_{\left(5,13.2^{1}-5\right)} \cong Q \mathscr{P}_{5}(\omega) \bigoplus Q \mathscr{P}_{5}\left(\omega^{\prime}\right)$, where $\omega=(3,3,1,1)$, and $\omega^{\prime}=(3,3,3)$. A routine computation shows that

$$
\mathscr{B}_{5}^{0}\left(13.2^{1}-5\right)=\bar{\Phi}\left(\mathscr{B}_{4}\left(13.2^{1}-5\right)\right)=\mathscr{B}_{5}^{0}(\omega) \cup \mathscr{B}_{5}^{0}\left(\omega^{\prime}\right) .
$$

Combining this with Propositions 3.3.6 and 3.3.7 gives

$$
\bar{\Phi}\left(\mathscr{B}_{4}(\omega)\right) \subset \mathscr{B}_{5}(\omega), \quad \bar{\Phi}\left(\mathscr{B}_{4}\left(\omega^{\prime}\right)\right) \subset \mathscr{B}_{5}\left(\omega^{\prime}\right) .
$$

Next, we have $\left(Q \mathscr{P}_{5}\right)_{13.2^{t}-5} \cong\left(Q \mathscr{P}_{5}\right)_{13.2^{2}-5}$ for all $t \geq 2$ and

$$
\operatorname{Ker}\left(\widetilde{S q_{*}^{0}}\right)_{\left(5,13.2^{2}-5\right)} \cong \bigoplus_{1 \leq k \leq 5} Q \mathscr{P}_{5}\left(\bar{\omega}_{(k)}\right) .
$$

From the above computations,

$$
\mathscr{B}_{5}^{0}\left(\bar{\omega}_{(1)}\right)=\mathscr{B}_{5}^{0}\left(13.2^{2}-5\right)=\bar{\Phi}\left(\mathscr{B}_{4}\left(13.2^{2}-5\right)\right)=\bar{\Phi}\left(\mathscr{B}_{4}\left(\bar{\omega}_{(1)}\right)\right) .
$$

Then, by Corollary 3.3.23, and Propositions 3.3.25, 3.3.33, 3.3.34, we conclude

$$
\bar{\Phi}\left(\mathscr{B}_{4}\left(\bar{\omega}_{(1)}\right)\right) \subset \mathscr{B}_{5}\left(\bar{\omega}_{(1)}\right) .
$$

If $\bar{\omega}$ is a weight vector of degree $13.2^{2}-5$ and $\bar{\omega} \neq \bar{\omega}_{(1)}$, then $\mathscr{B}_{4}(\bar{\omega})=\emptyset$. Furthermore, if $t>2$, then $\mathscr{B}_{4}\left(13.2^{t}-5\right)=\emptyset$.

From the above remarks, Conjecture 3.2.2 also satisfies in case of five variables and generic degree $13.2^{t}-5$ for $t$ an arbitrary non-negative integer.

\section{An application of Theorem 1.1}

The goal of this section is to prove Theorem 1.2. More precisely, by using our results in Sect.3 and a result in [70], we describe the $\mathbb{Z} / 2\left(G L_{d}\right)$-modules structure of $Q \mathscr{P}_{5}$ in degree $13.2^{t}-5$ for $t \in\{0,1\}$. Then, we explicitly determine all $G L_{5}$-invariants of these spaces.

Before coming to the proof of the theorem, we introduce some notations and homomorphisms. We note that $(\mathbb{Z} / 2)^{\times d}$ regarded as a $\mathbb{Z} / 2$-vector space of dimension $d$ and

$$
(\mathbb{Z} / 2)^{\times d} \cong\left\langle x_{1}, \ldots, x_{d}\right\rangle \subset \mathscr{P}_{d} .
$$

For $1 \leq t \leq d$, define the $\mathbb{Z} / 2$-linear map $\tau_{t}:(\mathbb{Z} / 2)^{\times d} \rightarrow(\mathbb{Z} / 2)^{\times d}$, which is determined by

$$
\tau_{t}\left(x_{t}\right)=x_{t+1}, \tau_{t}\left(x_{t+1}\right)=x_{t}, \tau_{t}\left(x_{m}\right)=x_{m} \quad(m \neq t, t+1,1 \leq t \leq d-1),
$$

and

$$
\tau_{d}\left(x_{1}\right)=x_{1}+x_{2}, \tau_{d}\left(x_{m}\right)=x_{m} \quad(m>1) .
$$

Denote by $S_{d}$ the symmetric group of degree $d$. Then, $S_{d}$ is generated by $\tau_{t}, 1 \leq t \leq d-1$. For each permutation in $S_{d}$, consider corresponding permutation matrix; these form a group of matrices isomorphic to $S_{d}$. So, $G L_{d}=G L(d, \mathbb{Z} / 2) \cong G L\left((\mathbb{Z} / 2)^{\times d}\right)$ is generated by $S_{d}$ and $\tau_{d}$. Let 
$X=x_{1}^{a_{1}} x_{2}^{a_{2}} \ldots x_{d}^{a_{d}}$ be an monomial in $\mathscr{P}_{d}$. Then, the weight vector $\omega(X)$ is invariant under the permutation of the generators $x_{j}, j=1,2, \ldots, d$; hence $Q \mathscr{P}_{d}(\omega)$ also has a $S_{d}$-module structure. We have a homomorphism $\tau_{t}: \mathscr{P}_{d} \rightarrow \mathscr{P}_{d}$ of algebras, which is induced by $\tau_{t}$. Hence, a class $[u]_{\omega} \in Q \mathscr{P}_{d}(\omega)$ is an $G L_{d}$-invariant if and only if $\tau_{t}(u)+u \equiv_{\omega} 0$ for $1 \leq t \leq d$. If $\tau_{t}(u)+u \equiv{ }_{\omega} 0$ for $1 \leq t \leq d-1$, then $[u]_{\omega}$ is an $S_{d}$-invariant. Note that $\operatorname{dim}\left(\left(Q P_{d}\right)_{n}^{G L_{d}}\right) \leq \sum_{\operatorname{deg}(\omega)=n} \operatorname{dim}\left(Q P_{d}(\omega)^{G L_{d}}\right)$ (see Sect.2).

Let $\omega$ be a weight vector of degree $n$ and let $y_{1}, y_{2}, \ldots, y_{s}$ be the monomials in $\mathscr{P}_{d}(\omega)$ for $s \geq 1$. We consider a subgroup $L \subseteq G L_{d}$ and denote by

$$
\begin{array}{ll}
L\left(y_{1}, y_{2}, \ldots, y_{s}\right) & =\left\{\sigma\left(y_{j}\right): \sigma \in L, 1 \leq j \leq s\right\} \subset \mathscr{P}_{d}(\omega), \\
{\left[\mathscr{B}\left(y_{1}, y_{2}, \ldots, y_{s}\right)\right]_{\omega}} & =\left[\mathscr{B}_{d}(\omega)\right]_{\omega} \cap\left\langle\left[L\left(y_{1}, y_{2}, \ldots, y_{s}\right)\right]_{\omega}\right\rangle, \\
\theta\left(y_{j}\right) & =\sum_{x \in \mathscr{B}_{d}(n) \cap L\left(y_{j}\right)} x,
\end{array}
$$

where $\left\langle\left[L\left(y_{1}, y_{2}, \ldots, y_{s}\right)\right]_{\omega}\right\rangle$ is the $L$-submodule of $Q \mathscr{P}_{d}(\omega)$ generated by the set

$$
\left\{\left[y_{1}\right]_{\omega},\left[y_{2}\right]_{\omega}, \ldots,\left[y_{s}\right]_{\omega}\right\} .
$$

Now, we have $13.2^{1}-5=8$, and $13.2^{1}-5=21$. Recall that Kameko's map $\left.\widetilde{\left(S q_{*}^{0}\right.}\right)_{(5,21)}$ : $\left(Q \mathscr{P}_{5}\right)_{21} \rightarrow\left(Q \mathscr{P}_{5}\right)_{8}$ is an epimorphism of $G L_{5}$-modules. So, to prove Theorem 1.2 , we need to compute $G L_{5}$-invariants of $\left(Q \mathscr{P}_{5}\right)_{8}$.

\subsection{Computation of $\left(Q \mathscr{P}_{5}\right)_{8}^{G L_{5}}$}

According to Tín [69], the $\mathbb{Z} / 2$-vector space $\left(Q \mathscr{P}_{5}\right)_{8}$ has the basis $\left[\left\{y_{8, i}: 1 \leq i \leq 174\right\}\right]$, where the monomials $y_{i}:=y_{8, i}, 1 \leq i \leq 174$, are given in Sect.6.1 of the online version [44].

Theorem 4.1.1. The space $\left(Q \mathscr{P}_{5}\right)^{G L_{5}}$ is trivial in degree 8 .

We prepare some lemmas for the proof of the theorem. We have

$$
\left(Q \mathscr{P}_{5}\right)_{8} \cong Q \mathscr{P}_{5}^{0}\left(\widetilde{\omega}_{(1)}\right) \bigoplus Q \mathscr{P}_{5}\left(\widetilde{\omega}_{(2)}\right) \bigoplus Q \mathscr{P}_{5}\left(\widetilde{\omega}_{(3)}\right),
$$

where $\widetilde{\omega}_{(1)}=(2,1,1), \widetilde{\omega}_{(2)}=(2,3), \widetilde{\omega}_{(3)}=(4,2)$. We see that $\operatorname{dim} Q \mathscr{P}_{5}^{0}\left(\widetilde{\omega}_{(1)}\right)=105$ with the basis $\bigcup_{1 \leq i \leq 6}\left[\mathscr{B}\left(y_{i}\right)\right]_{\widetilde{\omega}_{(1)}}$, where

$$
\begin{gathered}
y_{1}=x_{4} x_{5}^{7}, \quad y_{2}=x_{4}^{3} x_{5}^{5}, \quad y_{3}=x_{3} x_{4} x_{5}^{6}, \\
y_{4}=x_{3} x_{4}^{2} x_{5}^{5}, \quad y_{5}=x_{3} x_{4}^{3} x_{5}^{4}, \quad y_{6}=x_{2} x_{3} x_{4}^{2} x_{5}^{4} .
\end{gathered}
$$

Observe that $\widetilde{\omega}_{(1)}$ is the weight vector of the mimimal spike $x_{1}^{7} x_{2}$, so $[X]_{\widetilde{\omega}_{(1)}}=[X]$ for all $X \in\left(\mathscr{P}_{5}\right)_{8}$. A direct computation shows that

$$
\begin{array}{ll}
{\left[S_{5}\left(y_{1}\right)\right]} & =\left\langle\left[y_{i}\right]: 1 \leq i \leq 20\right\rangle, \\
{\left[S_{5}\left(y_{2}\right)\right]} & =\left\langle\left[y_{i}\right]: 21 \leq i \leq 30\right\rangle, \\
{\left[S_{5}\left(y_{3}, y_{4}, y_{5}\right)\right]} & =\left\langle\left[y_{i}\right]: 31 \leq i \leq 90\right\rangle, \\
{\left[S_{5}\left(y_{6}\right)\right]} & =\left\langle\left[y_{i}\right]: 91 \leq i \leq 105\right\rangle
\end{array}
$$

are $S_{5}$-submodules of $Q \mathscr{P}_{5}^{0}\left(\widetilde{\omega}_{(1)}\right)$. Hence, we have a direct summand decomposition of $S_{5}$-modules: $Q \mathscr{P}_{5}^{0}\left(\widetilde{\omega}_{(1)}\right)=\left[S_{5}\left(y_{1}\right)\right] \bigoplus\left[S_{5}\left(y_{2}\right)\right] \bigoplus\left[S_{5}\left(y_{3}, y_{4}, y_{5}\right)\right] \bigoplus\left[S_{5}\left(y_{6}\right)\right]$.

Lemma 4.1.2. $\left.Q \mathscr{P}_{5}^{0}\left(\widetilde{\omega}_{(1)}\right)\right)^{S_{5}}$ has dimension 4 .

Proof. We prove the following:

$$
\begin{array}{ll}
{\left[S_{5}\left(y_{j}\right)\right]^{S_{5}}} & =\left\langle\left[\theta\left(y_{j}\right)\right]\right\rangle, j=1,2,6 \\
{\left[S_{5}\left(y_{3}, y_{4}, y_{5}\right)\right]^{S_{5}}} & =\left\langle\left[q:=y_{31}+y_{32}+\cdots+y_{70}\right]\right\rangle .
\end{array}
$$

Indeed, we compute $\left[S_{5}\left(y_{j}\right)\right]^{S_{5}}$ for $j=2$ and $\left[S_{5}\left(y_{3}, y_{4}, y_{5}\right)\right]^{S_{5}}$. The others can be proved by a similar technique. Note that $\operatorname{dim}\left[S_{5}\left(y_{2}\right)\right]=10$ with a basis consisting of all the classes represented by the monomials $y_{i}: 21 \leq i \leq 30$. Suppose that $a=\sum_{21 \leq i \leq 30} \gamma_{i} y_{i}$ with $\gamma_{i} \in \mathbb{Z} / 2$ and $[a] \in$ $\left[S_{5}\left(y_{2}\right)\right]^{S_{5}}$. By a direct computation using Theorem 3.1.3, we have

$$
\begin{aligned}
\tau_{1}(a)+a & =\sum X \operatorname{modulo}\left(\mathcal{A}_{2}^{+} \mathscr{P}_{5}\right)=0 \operatorname{modulo}\left(\mathcal{A}_{2}^{+} \mathscr{P}_{5}\right), \\
\tau_{2}(a)+a & =\sum Y \operatorname{modulo}\left(\mathcal{A}_{2}^{+} \mathscr{P}_{5}\right)=0 \operatorname{modulo}\left(\mathcal{A}_{2}^{+} \mathscr{P}_{5}\right), \\
\tau_{3}(a)+a & =\sum Z \operatorname{modulo}\left(\mathcal{A}_{2}^{+} \mathscr{P}_{5}\right)=0 \operatorname{modulo}\left(\mathcal{A}_{2}^{+} \mathscr{P}_{5}\right), \\
\tau_{4}(a)+a & =\sum W \operatorname{modulo}\left(\mathcal{A}_{2}^{+} \mathscr{P}_{5}\right)=0 \operatorname{modulo}\left(\mathcal{A}_{2}^{+} \mathscr{P}_{5}\right),
\end{aligned}
$$


where

$$
\begin{aligned}
\sum X & =\left(\gamma_{24}+\gamma_{27}\right)\left(y_{24}+y_{27}\right)+\left(\gamma_{25}+\gamma_{28}\right)\left(y_{25}+y_{28}\right)+\left(\gamma_{26}+\gamma_{29}\right)\left(y_{26}+y_{29}\right), \\
\sum Y & =\left(\gamma_{22}+\gamma_{24}\right)\left(y_{22}+y_{24}\right)+\left(\gamma_{23}+\gamma_{25}\right)\left(y_{23}+y_{25}\right)+\left(\gamma_{29}+\gamma_{30}\right)\left(y_{29}+y_{30}\right), \\
\sum Z & =\left(\gamma_{21}+\gamma_{22}\right)\left(y_{21}+y_{22}\right)+\left(\gamma_{25}+\gamma_{26}\right)\left(y_{25}+y_{26}\right)+\left(\gamma_{28}+\gamma_{29}\right)\left(y_{28}+y_{29}\right), \\
\sum W & =\left(\gamma_{22}+\gamma_{23}\right)\left(y_{22}+y_{23}\right)+\left(\gamma_{24}+\gamma_{25}\right)\left(y_{24}+y_{25}\right)+\left(\gamma_{27}+\gamma_{28}\right)\left(y_{27}+y_{28}\right) .
\end{aligned}
$$

These relations imply that $\gamma_{i}=\gamma_{21}$ for $i=22, \ldots, 30$. Hence, we get $a=\theta\left(\mathfrak{y}_{2}\right) \operatorname{modulo}\left(\mathcal{A}_{2}^{+} \mathscr{P}_{5}\right)$ with $\theta\left(Y_{2}\right)=\sum_{21 \leq j \leq 30} y_{j}$.

Now, we have the set $\left.\left\{\left[y_{i}\right]: 31 \leq i \leq 90\right]\right\}$ is a basis of $\left[S_{5}\left(y_{3}, y_{4}, y_{5}\right)\right]$. Suppose that $b=$ $\sum_{31<i<90} \gamma_{i} y_{i}$ with $\gamma_{i} \in \mathbb{Z} / 2$ and $[b] \in\left[S_{5}\left(y_{3}, y_{4}, y_{5}\right)\right]^{S_{5}}$. By a similar computation from the relations $\tau_{t}(b)+b=0$ modulo $\left(\mathcal{A}_{2}^{+} \mathscr{P}_{5}\right), t=1,2,3,4$, one gets $\gamma_{i}=0$ for $71 \leq i \leq 90$ and $\gamma_{i}=\gamma_{31}, i=32,33, \ldots, 70$. This means $b=q \operatorname{modulo}\left(\mathcal{A}_{2}^{+} \mathscr{P}_{5}\right)$ with $q=\sum_{31 \leq i \leq 70}$. The lemma is proved.

Lemma 4.1.3. The subspace $\left(Q \mathscr{P}_{5}\left(\widetilde{\omega}_{(3)}\right)\right)^{G L_{5}}$ is trivial.

Proof. We see that $Q \mathscr{P}_{5}\left(\widetilde{\omega}_{(3)}\right)$ is the $\mathbb{Z} / 2$-vector space of dimension 45 with the basis $\left[\mathscr{B}\left(\mathcal{y}_{130}=\right.\right.$ $\left.\left.x_{2} x_{3} x_{4}^{3} x_{5}^{3}\right)\right] \widetilde{\omega}_{(3)} \cup\left[\mathscr{B}\left(y_{160}=x_{1} x_{2} x_{3} x_{4}^{2} x_{5}^{3}\right)\right]_{\tilde{\omega}_{(3)}}$. Furthermore, $\left[S_{5}\left(y_{130}\right)\right]$ and $\left[S_{5}\left(y_{160}\right)\right]$ are $S_{5}$-submodules of $Q \mathscr{P}_{5}^{0}\left(\widetilde{\omega}_{(3)}\right)$, where $\left[S_{5}\left(y_{130}\right)\right]_{\widetilde{\omega}_{(3)}}=\left\langle\left[y_{i}: 130 \leq i \leq 159\right]_{\left(\widetilde{\omega}_{(3)}\right)}\right\rangle$ and $\left[S_{5}\left(y_{160}\right)\right]_{\widetilde{\omega}_{(3)}}=\left\langle\left[y_{i}\right.\right.$ : $\left.160 \leq i \leq 174]_{\left(\widetilde{\omega}_{(3)}\right)}\right\rangle$. So, we have a direct summand decomposition of $S_{5}$-modules: $Q \mathscr{P}_{5}\left(\widetilde{\omega}_{(3)}\right)=$ $\left[S_{5}\left(y_{130}\right)\right]_{\widetilde{\omega}_{(3)}} \bigoplus\left[S_{5}\left(y_{160}\right)\right]_{\widetilde{\omega}_{(3)}}$. The set $\left[\mathscr{B}\left(y_{130}\right)\right]_{\widetilde{\omega}_{(3)}}$ is a basis of $\left[S_{5}\left(y_{130}\right)\right]_{\widetilde{\omega}_{(3)}}$. The action of $S_{5}$ on $Q \mathscr{P}_{5}$ induces the one of it on $\left[\mathscr{B}\left(y_{130}\right)\right]_{\tilde{\omega}_{(3)}}$. On the other hand, this action is transitive, hence if $a=\sum_{130 \leq i<159} \gamma_{i} y_{i}$ with $\gamma_{i} \in \mathbb{Z} / 2$ and $[a] \in\left[S_{5}\left(y_{130}\right)\right]^{S_{5}}$, then from the relations $\tau_{t}(a)+a \equiv_{\widetilde{\omega}_{(3)}} 0,1 \leq t \leq 4$, we get $\gamma_{i}=\gamma_{130}, \forall i, 131 \leq i \leq 159$. In other words, $\left[S_{5}\left(y_{130}\right)\right]_{\widetilde{\omega}_{(3)}}^{S_{5}}=\left\langle\left[\theta\left(y_{130}\right)\right]_{\widetilde{\omega}_{(3)}}\right\rangle$ with $\theta\left(y_{130}\right)=\sum_{130 \leq i \leq 159} y_{i}$.

Next, we have $\operatorname{dim}\left[S_{5}\left(y_{160}\right)\right]_{\widetilde{\omega}_{(3)}}=15$ with the basis $\left[\mathscr{B}\left(y_{160)}\right)\right]_{\widetilde{\omega}_{(3)}}$. Suppose $b=\sum_{160 \leq i \leq 174} \gamma_{j} y_{j}$ with $\gamma_{j} \in \mathbb{Z} / 2$ and $[b] \in\left[S_{5}\left(y_{160}\right)\right]^{S_{5}}$. A direct computation shows:

$$
\begin{aligned}
& \tau_{1}(b)+b \quad \equiv_{\widetilde{\omega}_{(3)}} \gamma_{169}\left(y_{160}+y_{162}\right)+\gamma_{170}\left(y_{161}+y_{163}\right)+\gamma_{171}\left(y_{164}+y_{165}\right) \\
& +\left(\gamma_{166}+\gamma_{172}\right)\left(y_{166}+y_{172}\right)+\left(\gamma_{167}+\gamma_{173}\right)\left(y_{167}+y_{173}\right) \\
& +\left(\gamma_{168}+\gamma_{174}\right)\left(y_{168}+y_{174}\right) \\
& \tau_{2}(b)+b \equiv \widetilde{\omega}_{(3)}\left(\gamma_{162}+\gamma_{169}\right)\left(y_{162}+y_{169}\right)+\left(\gamma_{163}+\gamma_{170}\right)\left(y_{163}+y_{170}\right) \\
& +\left(\gamma_{164}+\gamma_{166}\right)\left(y_{164}+y_{166}\right)+\left(\gamma_{165}+\gamma_{167}\right)\left(y_{165}+y_{167}\right) \\
& +\left(\gamma_{168}+\gamma_{171}\right)\left(y_{168}+y_{171}\right)+\left(\gamma_{165}+\gamma_{167}\right)\left(y_{165}+y_{167}\right) \\
& +\gamma_{174}\left(y_{172}+y_{173}\right) \text {, } \\
& \tau_{3}(b)+b \equiv \widetilde{\omega}_{(3)}\left(\gamma_{160}+\gamma_{162}\right)\left(y_{160}+y_{162}\right)+\left(\gamma_{161}+\gamma_{164}\right)\left(y_{161}+y_{164}\right) \\
& +\left(\gamma_{163}+\gamma_{165}\right)\left(y_{163}+y_{165}\right)+\left(\gamma_{167}+\gamma_{168}\right)\left(y_{167}+y_{168}\right) \\
& +\left(\gamma_{170}+\gamma_{171}\right)\left(y_{170}+y_{171}\right)+\left(\gamma_{173}+\gamma_{174}\right)\left(y_{173}+y_{174}\right), \\
& \tau_{4}(b)+b \equiv \equiv_{\widetilde{\omega}_{(3)}}\left(\gamma_{160}+\gamma_{161}\right)\left(y_{160}+y_{161}\right)+\left(\gamma_{162}+\gamma_{163}\right)\left(y_{162}+y_{163}\right) \\
& +\left(\gamma_{164}+\gamma_{165}\right)\left(y_{164}+y_{165}\right)+\left(\gamma_{166}+\gamma_{167}\right)\left(y_{166}+y_{167}\right) \\
& +\left(\gamma_{169}+\gamma_{170}\right)\left(y_{169}+y_{170}\right)+\left(\gamma_{172}+\gamma_{173}\right)\left(y_{172}+y_{173}\right) \text {. }
\end{aligned}
$$

Then, from the relations $\tau_{t}(b)+$ bequiv $_{\widetilde{\omega}_{(3)}} 0$, we coclude $\gamma_{j}=0, \forall j$.

Now, let $[X]_{\widetilde{\omega}_{(3)}} \in\left(Q \mathscr{P}_{5}\left(\widetilde{\omega}_{(3)}\right)\right)^{G L_{5}}$ with $X \in \mathscr{P}_{5}\left(\widetilde{\omega}_{(3)}\right)$, then $[X]_{\widetilde{\omega}_{(3)}} \in\left(Q \mathscr{P}_{5}\left(\widetilde{\omega}_{(3)}\right)\right)^{S_{5}}$. So, we have $X \equiv_{\widetilde{\omega}_{(3)}} \gamma \theta\left(y_{130}\right)$ with $\gamma \in \mathbb{Z} / 2$. By a direct computation, we obtain

$$
\tau_{5}(X)+X \equiv_{\widetilde{\omega}_{(3)}} \gamma y_{130}+\text { other terms } \equiv_{\widetilde{\omega}_{(3)}} 0 .
$$

This implies $\gamma=0$. The proposition follows.

By a simple computation using the techniques as given in the proof of Lemmas 4.1.2 and 4.1.3, we claim that

Lemma 4.1.4. The following results are true:

i) We have a direct summand decomposition of the $S_{5}$-modules:

$$
Q \mathscr{P}_{5}\left(\widetilde{\omega}_{(2)}\right)=\left[S_{5}\left(y_{106}\right)\right]_{\tilde{\omega}_{(2)}} \bigoplus\left[S_{5}\left(y_{126}\right)\right] \widetilde{\omega}_{(2)} .
$$

ii) The subspace $\left(Q \mathscr{P}_{5}\left(\widetilde{\omega}_{(2)}\right)\right)^{G L_{5}}$ is trivial.

Proof of Theorem 4.1.1. Let $[X] \in\left(Q \mathscr{P}_{5}\right)_{8}^{G L_{5}}$. Then, from Lemmas 4.1.2 - 4.1.4, we have

$$
X=\beta_{1} \theta\left(y_{1}\right)+\beta_{2} \theta\left(y_{2}\right)+\beta_{3} q+\beta_{4} \theta\left(y_{3}\right) \operatorname{modulo}\left(\mathcal{A}_{2}^{+} \mathscr{P}_{5}\right)
$$

with $\beta_{t} \in \mathbb{Z} / 2,1 \leq t \leq 4$. By using Theorem 3.1.3 and computing $\tau_{5}(X)+X$ in terms of the admissible monomials (modulo $\left(\mathcal{A}_{2}^{+} \mathscr{P}_{5}\right)$ ), we conclude

$$
\tau_{5}(X)+X \equiv \beta_{1} y_{7}+\left(\beta_{1}+\beta_{2}\right) y_{16}+\beta_{3} y_{33}+\beta_{4}\left(y_{91}+y_{92}+y_{93}\right)+\text { other terms } \equiv 0 .
$$


This relation shows $\beta_{1}=\beta_{2}=\beta_{3}=\beta_{4}=0$. The proposition is proved.

As an immediate consequence of Theorem 4.1.1, we get the following.

Corollary 4.1.5. The fifth algebraic transfer

$$
\operatorname{Tr}_{5}: \mathbb{Z} / 2 \otimes_{G L_{5}} P_{\mathcal{A}_{2}} H_{8}\left(B(\mathbb{Z} / 2)^{\times 5}\right) \rightarrow \operatorname{Ext}_{\mathcal{A}_{2}}^{5,5+8}(\mathbb{Z} / 2, \mathbb{Z} / 2)
$$

is a trivial isomorphism.

\subsection{Computation of $\left(\operatorname{Ker}\left(\widetilde{S q_{*}^{0}}\right)_{(5,21)}\right)^{S_{5}}$}

From the results in Sect.3.3.1, we see that $\operatorname{dim} \operatorname{Ker}\left(\widetilde{S q_{*}^{0}}\right)_{(5,21)}=666$ with the basis $\left\{\left[y_{21, t}\right]: 1 \leq\right.$ $t \leq 666\}$. Here, the admissible monomials $y_{t}:=y_{21, t}, 1 \leq t \leq 666$, is described in Sects.6.6 and 6.7 of the online version [44]. Recall that $\left(\widetilde{S q_{*}^{0}}\right)_{(5,21)}$ is an epimorphism of $G L_{5}$-modules. Combining this and the results in Sect.4.1, we get $\left(Q \mathscr{P}_{5}\right)_{21}^{G L_{5}} \subseteq\left(\operatorname{Ker}\left(\widetilde{S q_{*}^{0}}\right)_{(5,21)}\right)^{G L_{5}}$. By Lemma 3.3.2, we have a direct summand decomposition of the $S_{5}$-modules:

$$
\operatorname{Ker}\left(\widetilde{S q_{*}^{0}}\right)_{(5,21)}=Q \mathscr{P}_{5}(3,3,1,1) \bigoplus Q \mathscr{P}_{5}(3,3,3) .
$$

For $\omega=(3,3,1,1)$, according to the results in Sect.3.3.1, we get $Q \mathscr{P}_{5}(\omega)=Q \mathscr{P}_{5}^{0}(\omega) \bigoplus Q \mathscr{P}_{5}^{+}(\omega)$ with $\operatorname{dim} Q \mathscr{P}_{5}^{0}(\omega)=340$ and $\operatorname{dim} Q \mathscr{P}_{5}^{+}(\omega)=196$. Note that $\mathscr{Z}=x_{1}^{15} x_{2}^{3} x_{3}^{3}$ is the minimal spike monomial in $\left(\mathscr{P}_{5}\right)_{21}$ and $\omega(\mathscr{Z})=\omega$. So, $[x]_{\omega}=[x]$ for any $x \in\left(\mathscr{P}_{5}\right)_{21}$. By using the results in Sect.3.3.1, we see that there is a direct summand decomposition of the $S_{5}$-modules:

$$
Q \mathscr{P}_{5}^{0}(\omega)=\left\langle\left[S_{5}\left(y_{1}\right)\right]\right\rangle \bigoplus\left\langle\left[S_{5}\left(y_{31}\right)\right]\right\rangle \bigoplus\left\langle\left[S_{5}\left(y_{61}\right)\right]\right\rangle \bigoplus\left\langle\left[S_{5}\left(y_{121}\right)\right]\right\rangle \bigoplus\left\langle\left[\mathbb{V}_{1}\right]\right\rangle,
$$

where

$$
\begin{aligned}
\mathscr{B}\left(y_{1}\right) & =\left\{y_{t}: 1 \leq t \leq 30\right\}, \quad \mathscr{B}\left(y_{31}\right)=\left\{y_{t}: 31 \leq t \leq 60\right\} \\
\mathscr{B}\left(y_{61}\right) & =\left\{y_{t}: 61 \leq t \leq 120\right\}, \quad \mathscr{B}\left(y_{121}\right)=\left\{y_{t}: 121 \leq t \leq 150\right\} \\
\mathbb{V}_{1} & =\mathscr{B}\left(y_{151}, y_{181}, y_{201}, y_{241}, y_{256}, y_{266}, y_{286}, y_{296}, y_{301}, y_{316}\right) \\
& =\left\{y_{t}: 151 \leq t \leq 340\right\}
\end{aligned}
$$

Lemma 4.2.1. The following results are true:

a) $\left\langle\left[S_{5}\left(y_{j}\right)\right]\right\rangle^{S_{5}}=\left\langle\left[\theta\left(y_{j}\right)\right]\right\rangle$ for $j=1,31,61,121$.

b) The subspace $\left\langle\left[S_{5}\left(\mathbb{V}_{1}\right)\right]\right\rangle^{S_{5}}$ is trivial.

Outline of the proof. For $j=1$, let $\left[f_{1}\right] \in\left\langle\left[S_{5}\left(y_{1}\right)\right]\right\rangle^{S_{5}}$. Then, we have

$$
\tau_{m}\left(f_{1}\right)=\sum_{X \in \mathscr{B}\left(y_{1}\right)} \beta_{X} \cdot X \operatorname{modulo}\left(\mathcal{A}_{2}^{+} \mathscr{P}_{5}\right), 0<m<5
$$

with $f_{1}=\sum_{X \in \mathscr{B}\left(y_{1}\right)} \beta_{X} \cdot X$ and $\beta_{X} \in \mathbb{Z} / 2$. By a direct computation, we can see that the action of the symmetric group $S_{5}$ on $Q \mathscr{P}_{5}$ induces the one of it on the set $\left[\mathscr{B}\left(y_{j}\right)\right]$ and this action is transitive. So, we get $\beta_{X}=\beta_{X^{\prime}}=\beta \in \mathbb{Z} / 2$ for all $X, X^{\prime} \in \mathscr{B}\left(y_{j}\right)$. This means $f_{1}=\theta\left(y_{1}\right) \operatorname{modulo}\left(\mathcal{A}_{2}^{+} \mathscr{P}_{5}\right)$. For $j=31,61,121$, we determine $\tau_{m}\left(f_{j}\right)+f_{j}$ in terms of $y_{j}$. Then, by a simple computation using the relations $\tau_{m}\left(f_{j}\right)=f_{j} \operatorname{modulo}\left(\mathcal{A}_{2}^{+} \mathscr{P}_{5}\right)$, we conclude $f_{j}=\theta\left(y_{j}\right) \operatorname{modulo}\left(\mathcal{A}_{2}^{+} \mathscr{P}_{5}\right)$.

Next, we have $\operatorname{dim}\left\langle\mathbb{V}_{1}\right\rangle=190$ with the basis $\left\{\left[y_{t}\right]: 151 \leq t \leq 340\right\}$. Assume that $g=$ $\sum_{u \in \mathbb{V}_{1}} \gamma_{u} . u$ with $\gamma_{u} \in \mathbb{Z} / 2$ and $[g] \in\left\langle\left[\mathbb{V}_{1}\right]\right\rangle^{S_{5}}$. By using Theorem 3.1.3 and a similar computation as given in the proof of Lemmas 4.1 .2 and 4.1.3, we obtain $\gamma_{u}=0$ for all $u \in \mathbb{V}_{1}$. This implies that $g$ is $\mathcal{A}_{2}$-decomposable. The lemma follows.

Lemma 4.2.2. $Q \mathscr{P}_{5}^{+}(\omega)^{S_{5}}=\left\langle\left[p:=y_{411}+y_{412}+\cdots+y_{419}\right]\right\rangle$.

Proof. From Proposition 3.3.6, we see that the sets $\left[S_{5}\left(y_{401}\right)\right]=\left\langle\left[y_{t}\right]: 401 \leq t \leq 410\right\rangle$ and $\mathbb{V}_{2}=\left\langle\left[y_{t}\right]: 411 \leq t \leq 596\right\rangle$, are $S_{5}$-submodules of $Q \mathscr{P}_{5}^{+}(\omega)$. Hence, we have a direct summand decomposition of the $S_{5}$-modules:

$$
Q \mathscr{P}_{5}^{+}(\omega)=\left[S_{5}\left(y_{401}\right)\right] \bigoplus \mathbb{V}_{2} .
$$

The set $\left[\mathscr{B}\left(y_{401}\right)\right]$ is a basis of $\left[S_{5}\left(y_{401}\right)\right]$. Assume that $Z$ is a polynomial such that $[Z] \in\left[S_{5}\left(y_{401}\right)\right]^{S_{5}}$ and $Z=\sum_{401<t<410} \ell_{t} y_{t}$ with $\ell_{t} \in \mathbb{Z} / 2$. For $1 \leq j \leq 4$, we explicitly compute $\tau_{j}(Z)+Z$ in the terms of the admissible monomials $y_{t}, 401 \leq t \leq 410$. 
By a direct computation using Theorem 3.1.3, we get

$$
\begin{aligned}
\tau_{1}(Z)+Z \equiv & \ell_{401} y_{404}+\ell_{402} y_{405}+\ell_{403} y_{407}+\left(\ell_{406}+\ell_{409}\right)\left(y_{406}+y_{409}\right) \\
& +\left(\ell_{408}+\ell_{410}\right)\left(y_{408}+y_{410}\right), \\
\tau_{2}(Z)+Z \equiv & \left(\ell_{401}+\ell_{404}\right)\left(y_{401}+y_{404}\right)+\left(\ell_{402}+\ell_{405}\right)\left(y_{402}+y_{405}\right) \\
& +\left(\ell_{403}+\ell_{406}\right)\left(y_{403}+y_{406}\right)+\left(\ell_{407}+\ell_{408}\right)\left(y_{407}+y_{408}\right)+\ell_{409} y_{410}, \\
\tau_{3}(Z)+Z \equiv & \ell_{401} y_{404}+\left(\ell_{402}+\ell_{403}\right)\left(y_{402}+y_{403}\right)+\left(\ell_{405}+\ell_{407}\right)\left(y_{405}+y_{407}\right), \\
& +\left(\ell_{406}+\ell_{408}\right)\left(y_{406}+y_{408}\right)+\left(\ell_{409}+\ell_{410}\right)\left(y_{409}+y_{410}\right), \\
\tau_{4}(Z)+Z \equiv & \left(\ell_{401}+\ell_{402}\right)\left(y_{401}+y_{402}\right)+\ell_{403} y_{407}+\left(\ell_{404}+\ell_{405}\right)\left(y_{404}+y_{405}\right) \\
& +\ell_{406} y_{408}+\ell_{409} y_{410} .
\end{aligned}
$$

Then, by the relations $\tau_{j}(Z)=Z \operatorname{modulo}\left(\mathcal{A}_{2}^{+} \mathscr{P}_{5}\right), j=1,2,3,4$, one gets $\ell_{t}=0, \forall t$. This implies that $Z$ is $\mathcal{A}_{2}$-decomposable.

Note that $\operatorname{dim} \mathbb{V}_{2}=186$ with the basis $\left\{\left[y_{j}\right]: 411 \leq j \leq 596\right\}$. Then if the polynomial $g=\sum_{u \in \mathbb{V}_{2}} \sigma_{u} . u, \sigma_{u} \in \mathbb{Z} / 2$ such that $[g] \in \mathbb{V}_{2}^{S_{5}}$, then by a similar argument as given above, we obtain $g=p$ modulo $\left(\mathcal{A}_{2}^{+} \mathscr{P}_{5}\right)$. This completes the proof of the lemma.

Proposition 4.2.3. For $\bar{\omega}=(3,3,3)$, we have $Q \mathscr{P}_{5}(\bar{\omega})^{G L_{5}}=\left\langle\left[q_{2}\right]_{\bar{\omega}}\right\rangle$, where

$$
\begin{aligned}
q_{2}= & y_{652}+y_{653}+y_{654}+y_{656}+y_{657}+y_{658}+\sum_{661 \leq t \leq 666} y_{t} \\
= & x_{1} x_{2}^{6} x_{3}^{3} x_{4}^{5} x_{5}^{6}+x_{1} x_{2}^{3} x_{3}^{6} x_{4}^{5} x_{5}^{6}+x_{1} x_{2}^{3} x_{3}^{5} x_{4}^{6} x_{5}^{6}+x_{1}^{3} x_{2} x_{3}^{5} x_{4}^{6} x_{5}^{6} \\
& +x_{1}^{3} x_{2}^{5} x_{3} x_{4}^{6} x_{5}^{6}+x_{1}^{3} x_{2}^{5} x_{3}^{6} x_{4} x_{5}^{6}+x_{1}^{3} x_{2}^{3} x_{3}^{5} x_{4}^{4} x_{5}^{6}+x_{1}^{3} x_{2}^{3} x_{3}^{4} x_{4}^{5} x_{5}^{6} \\
& +x_{1}^{3} x_{2}^{3} x_{3}^{5} x_{4}^{6} x_{5}^{4}+x_{1}^{3} x_{2}^{4} x_{3}^{3} x_{4}^{5} x_{5}^{6}+x_{1}^{3} x_{2}^{5} x_{3}^{3} x_{4}^{6} x_{5}^{4}+x_{1}^{3} x_{2}^{5} x_{3}^{6} x_{4}^{3} x_{5}^{4} .
\end{aligned}
$$

Based on the results in Sect.3.3, we have

$$
\operatorname{dim} Q \mathscr{P}_{5}(\bar{\omega})=\operatorname{dim} Q \mathscr{P}_{5}^{0}(\bar{\omega})+\operatorname{dim} Q \mathscr{P}_{5}^{+}(\bar{\omega})=60+70=130 .
$$

Consider the following monomials:

$$
\begin{array}{llll}
y_{341}=x_{3}^{7} x_{4}^{7} x_{5}^{7}, & y_{351}=x_{2} x_{3}^{6} x_{4}^{7} x_{5}^{7}, & y_{381}=x_{2}^{3} x_{3}^{5} x_{4}^{6} x_{5}^{7}, \\
y_{597}=x_{1} x_{2}^{2} x_{3}^{4} x_{4}^{7} x_{5}^{7}, & y_{607}=x_{1} x_{2}^{6} x_{3} x_{4}^{6} x_{5}^{7}, & y_{617}=x_{1} x_{2}^{2} x_{3}^{5} x_{4}^{6} x_{5}^{7}, \\
y_{622}=x_{1} x_{2}^{3} x_{3}^{4} x_{4}^{6} x_{5}^{7}, & y_{642}=x_{1}^{3} x_{2}^{5} x_{3}^{2} x_{4}^{4} x_{5}^{7}, & y_{647}=x_{1}^{3} x_{2}^{3} x_{3}^{4} x_{4}^{4} x_{5}^{7}, \\
y_{652}=x_{1} x_{2}^{6} x_{3}^{3} x_{4}^{5} x_{5}^{6}, & y_{659}=x_{1}^{3} x_{2}^{5} x_{3}^{2} x_{4}^{5} x_{5}^{6}, & & y_{660}=x_{1}^{3} x_{2}^{5} x_{3}^{3} x_{4}^{4} x_{5}^{6} .
\end{array}
$$

The following lemma can be easily proved by a direct computation.

\section{Lemma 4.2.4.}

i) The following subspaces are $S_{5}$-submodules of $Q \mathscr{P}_{5}(\bar{\omega})$ :

$$
\begin{gathered}
\left\langle\left[S_{5}\left(y_{a}\right)\right]_{\bar{\omega}}\right\rangle, a=341,351,381,597, \mathbb{V}_{3}:=\left\langle\left[S_{5}\left(y_{607}, y_{617}, y_{622}, y_{642}, y_{647}\right)\right]_{\bar{\omega}}\right\rangle, \\
\mathbb{V}_{4}:=\left\langle\left[S_{5}\left(y_{652}, y_{659}, y_{660}\right)\right]_{\bar{\omega}}\right\rangle .
\end{gathered}
$$

ii) We have a direct summand decomposition of the $S_{5}$-modules:

$$
\begin{aligned}
Q \mathscr{P}_{5}(\bar{\omega})= & \left\langle\left[S_{5}\left(y_{341}\right)\right]_{\bar{\omega}}\right\rangle \bigoplus\left\langle\left[S_{5}\left(y_{351}\right)\right]_{\bar{\omega}}\right\rangle \bigoplus\left\langle\left[S_{5}\left(y_{381}\right)\right]_{\bar{\omega}}\right\rangle \\
& \bigoplus\left\langle\left[S_{5}\left(y_{597}\right)\right]_{\bar{\omega}} \bigoplus \bigoplus \mathbb{V}_{3} \bigoplus \mathbb{V}_{4} .\right.
\end{aligned}
$$

Lemma 4.2.5. We have the following results:

i) $\left\langle\left[S_{5}\left(y_{a}\right)\right]_{\bar{\omega}}\right\rangle^{S_{5}}=\left\langle\left[\theta\left(y_{a}\right)\right]_{\bar{\omega}}\right\rangle$, for $a=341,351,381,597$.

ii) $\left\langle\left[S_{5}\left(\mathbb{V}_{3}\right)\right]_{\bar{\omega}}\right\rangle^{S_{5}}=\left\langle\left[q_{1}:=y_{610}+y_{611}+y_{613}+y_{614}+y_{616}+\sum_{647<t<651} y_{t}\right]_{\bar{\omega}}\right\rangle$.

iii) $\left\langle\left[S_{5}\left(\mathbb{V}_{4}\right)\right]_{\bar{\omega}}\right\rangle^{S_{5}}=.\left\langle\left[q_{2}:=y_{652}+y_{653}+y_{654}+y_{656}+y_{657}+y_{658}+\sum_{661 \leq t \leq 666} y_{t}\right]_{\bar{\omega}}\right\rangle$,

The proof of the lemma is straightforward.

Proof of Proposition 4.2.3. By Lemmas 4.2.4 and 4.2.5, we get

$$
Q \mathscr{P}_{5}(\bar{\omega})^{S_{5}}=\left\langle\left[\theta\left(y_{341}\right)\right]_{\bar{\omega}},\left[\theta\left(y_{351}\right)\right]_{\bar{\omega}},\left[\theta\left(y_{381}\right)\right]_{\bar{\omega}},\left[\theta\left(y_{597}\right)\right]_{\bar{\omega}},\left[q_{1}\right]_{\bar{\omega}},\left[q_{2}\right]_{\bar{\omega}}\right\rangle
$$

Let $X$ be a polynomial in $\mathscr{P}_{5}(\bar{\omega})$ such that $[X]_{\bar{\omega}} \in Q \mathscr{P}_{5}(\bar{\omega})^{G L_{5}}$. Then, we have

$$
X \equiv_{\bar{\omega}} v_{1} \theta\left(y_{341}\right)+v_{2} \theta\left(y_{351}\right)+v_{3} \theta\left(y_{381}\right)+v_{4} \theta\left(y_{597}\right)+v_{5} q_{1}+v_{6} q_{2},
$$

with $v_{j} \in \mathbb{Z} / 2$ for $1 \leq j \leq 6$. We explicitly compute $\tau_{5}(X)$ in terms of the admissible monomials $y_{t}$ with $t=341,342, \ldots, 400,597,598, \ldots, 666$. By a direct computation, one gets

$$
\begin{aligned}
\tau_{5}(X)+X \equiv & \bar{\omega}_{\bar{\omega}}\left(v_{1}+v_{2}\right) y_{342}+\left(v_{2}+v_{4}\right) y_{351}+v_{2} y_{354}+v_{3} y_{383} \\
& +v_{5} y_{650}+\text { other terms . }
\end{aligned}
$$

Since $[X]_{\bar{\omega}} \in Q \mathscr{P}_{5}(\bar{\omega})^{G L_{5}}, v_{j}=0,1 \leq j \leq 5$. The proposition is proved. 
Combining Lemmas 4.2.1, 4.2.2 and 4.2.5 gives

Corollary 4.2.6. There exist exactly 11 non-zero classes in the kernel of $\left(\widetilde{S q_{*}^{0}}\right)_{(5,21)}$ invariant under the action of $S_{5}$.

\subsection{Proof of Theorem 1.2}

Suppose that $[T] \in\left(Q \mathscr{P}_{5}\right)_{21}^{G L_{5}}$ with $T$ is a polynomial in $\left(\mathscr{P}_{5}\right)_{21}$. From Proposition 4.2 .3 , we have $T=T^{*}+\zeta_{6} q_{2}$ modulo $\left(\mathcal{A}_{2}^{+} \mathscr{P}_{5}\right)$ with $T^{*} \in \mathscr{P}_{5}^{-}(\bar{\omega})$ and $\zeta_{6} \in \mathbb{Z} / 2$. By a simple computation, we see that $\left[q_{2}\right] \in\left(Q \mathscr{P}_{5}\right)_{21}^{S_{5}}$. This implies that $\left[T^{*}\right]$ is an $S_{5}$-invariant. On the other hand, $\left[\mathscr{P}_{5}^{-}(\bar{\omega})\right]=$ $Q \mathscr{P}_{5}(\omega)$. Hence, by Lemmas 4.2.1 and 4.2.2, we obtain

$$
T^{*}=\zeta_{1} \theta\left(y_{1}\right)+\zeta_{2} \theta\left(y_{31}\right)+\zeta_{3} \theta\left(y_{61}\right)+\zeta_{4} \theta\left(y_{121}\right)+\zeta_{5} p \operatorname{modulo}\left(\mathcal{A}_{2}^{+} \mathscr{P}_{5}\right),
$$

where $\zeta_{i} \in \mathbb{Z} / 2$. Using Theorem 1.1 and computing $\tau_{5}(T)+T$ in terms of the admissible monomials $y_{t}, 1 \leq t \leq 666$, we conclude

$$
\begin{aligned}
\tau_{5}(T)+T=\left(\zeta_{1}+\zeta_{3}\right) & y_{4}+\left(\zeta_{2}+\zeta_{4}\right) y_{35}+\zeta_{3} y_{61} \\
& +\left(\zeta_{3}+\zeta_{4}\right) y_{107}+\zeta_{5} y_{153}+\text { other terms } \operatorname{modulo}\left(\mathcal{A}_{2}^{+} \mathscr{P}_{5}\right) .
\end{aligned}
$$

By the relation $\tau_{5}(T)=T \operatorname{modulo}\left(\mathcal{A}_{2}^{+} \mathscr{P}_{5}\right)$, one gets $\zeta_{i}=0,1 \leq i \leq 5$. This shows that

$$
T=\zeta_{6} q_{2} \operatorname{modulo}\left(\mathcal{A}_{2}^{+} \mathscr{P}_{5}\right) .
$$

The proof of the theorem is completed.

\section{Proof of Theorem 1.3}

Obviously, $\lambda_{3} \in \Lambda^{1,3}$ and $\bar{f}_{0}=\lambda_{3} \lambda_{5} \lambda_{6} \lambda_{4}+\lambda_{3}^{2} \lambda_{7} \lambda_{5}+\lambda_{7} \lambda_{5} \lambda_{3}^{2}+\lambda_{7} \lambda_{5} \lambda_{4} \lambda_{2} \in \Lambda^{4,18}$ are the cycles in the lambda algebra $\Lambda$. By Lin [23], we have $\operatorname{Ext}_{\mathcal{A}_{2}}^{5,5+21}(\mathbb{Z} / 2, \mathbb{Z} / 2)=\left\langle h_{2} f_{0}\right\rangle$, where

$$
h_{2}=\left[\lambda_{3}\right] \in \operatorname{Ext}_{\mathcal{A}_{2}}^{1,4}(\mathbb{Z} / 2, \mathbb{Z} / 2), \text { and } f_{0}=\left[\bar{f}_{0}\right] \in \operatorname{Ext}_{\mathcal{A}_{2}}^{4,22}(\mathbb{Z} / 2, \mathbb{Z} / 2) \text {. }
$$

We note that $h_{2} f_{0}=h_{1} g_{1}$ with $h_{1} \in \operatorname{Ext}_{\mathcal{A}_{2}}^{1,2}(\mathbb{Z} / 2, \mathbb{Z} / 2)$ and $g_{1} \in \operatorname{Ext}_{\mathcal{A}_{2}}^{4,24}(\mathbb{Z} / 2, \mathbb{Z} / 2)$. By direct computations, we find that the following element is $\mathcal{A}_{2}^{+}$-annihilated in $H_{21}\left(B(\mathbb{Z} / 2)^{\times 5}\right)$ :

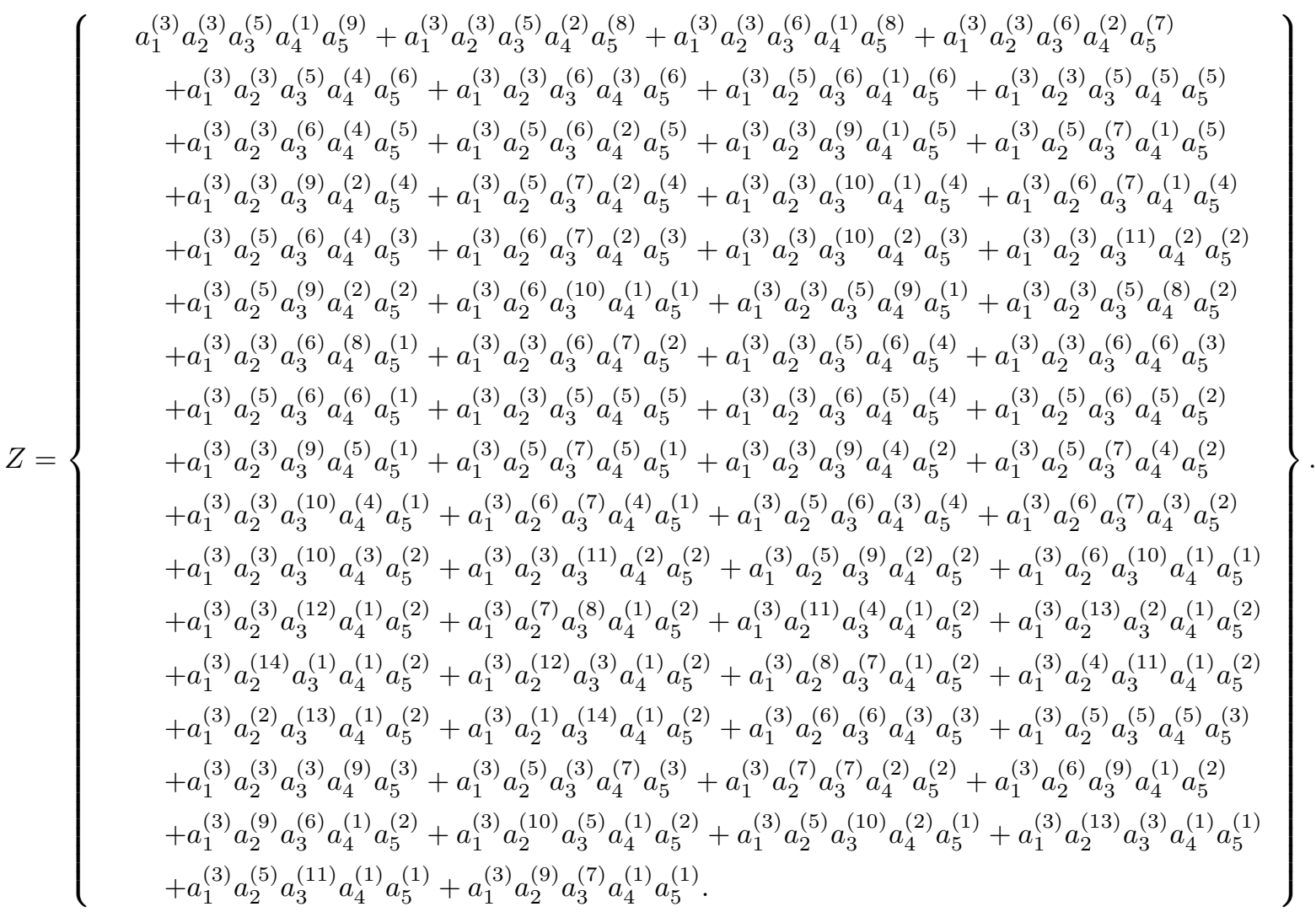

According to the proof of Theorem 1.2, $\left\{\left[q_{2}\right]\right\}$ is a basis of $\left(Q \mathscr{P}_{5}\right)^{G L_{5}}$ in degree $13.2^{1}-5$. Obviously, $\left\langle\left[q_{2}\right],[Z]\right\rangle=1$. Since $Z \in P_{\mathcal{A}_{2}} H_{13.2^{1}-5}\left(B(\mathbb{Z} / 2)^{\times 5}\right),[Z]$ is dual to $\left[q_{2}\right]$. Using the representation of $\operatorname{Tr}_{5}$ over the algebra $\Lambda$ and the differential (1.3) in Sect.1, we obtain

$$
\begin{aligned}
\psi_{5}(Z)= & \lambda_{3}^{2} \lambda_{5} \lambda_{6} \lambda_{4}+\lambda_{3}^{3} \lambda_{7} \lambda_{5}+\lambda_{3} \lambda_{7} \lambda_{5} \lambda_{2} \lambda_{3}^{2} \\
& +\lambda_{3} \lambda_{7} \lambda_{5} \lambda_{4} \lambda_{2}+\lambda_{3}^{2} \lambda_{5} \lambda_{3} \lambda_{7} \\
= & \lambda_{3} \bar{f}_{0}+\partial\left(\lambda_{3}^{2} \lambda_{5} \lambda_{11}\right) .
\end{aligned}
$$


Since $Z \in P_{\mathcal{A}_{2}} H_{13.2^{1}-5}\left(B(\mathbb{Z} / 2)^{\times 5}\right), \psi_{5}(Z)$ is a cycle in $\Lambda^{5,21}$. This implies that $h_{2} f_{0}$ is in the image of $\operatorname{Tr}_{5}$. Further, by Theorem $1.2, \mathbb{Z} / 2 \otimes_{G L_{5}} P_{\mathcal{A}_{2}} H_{13.2^{1}-5}\left(B(\mathbb{Z} / 2)^{\times 5}\right)$ and $\operatorname{Ext}_{\mathcal{A}_{2}}^{5,5+\left(13.2^{1}-5\right)}(\mathbb{Z} / 2, \mathbb{Z} / 2)$ that have the same dimensions are 1 . Hence, $\operatorname{Tr}_{5}$ is an isomorphism when acting on the space $\mathbb{Z} / 2 \otimes_{G L_{5}} P_{\mathcal{A}_{2}} H_{13.2^{1-5}}\left(B(\mathbb{Z} / 2)^{\times 5}\right)$. Theorem 1.3 is proved.

\section{References}

[1] J.F. Adams, On the structure and applications of the Steenrod algebra, Comment. Math. Helv. 32 (1958), no. 1, 180-214. https://doi.org/10.1007/BF02564578, available online at https://link.springer.com/article/ $10.1007 \% 2$ FBF02564578\#citeas

[2] _ On the non-existence of elements of Hopf invariant one, Ann. of Math. (2) 72 (1960), no. 1, 20-104. https://doi.org/10.2307/1970147

[3] J. Adem, The iteration of the Steenrod squares in Algebraic Topology, Proc. Natl. Acad. Sci. USA 38 (1952), no. 8, 20-726. https://doi.org/10.1073/pnas.38.8.720

[4] M.A. Alghamdi, M.C. Crabb and J.R. Hubbuck, Representations of the homology of BV and the Steenrod algebra I, in Adams Memorial Symposium on Algebraic Topology 2, N. Ray and G. Walker (ed.), London Math. Soc. Lecture Note Ser. 176 (1992), 217-234. https://doi.org/10.1017/CB09780511526312.020

[5] S.V. Ault and W. Singer, On the homology of elementary Abelian groups as modules over the Steenrod algebra, J. Pure Appl. Algebra 215 (2011), no. 12, 2847-2852. https://doi.org/10.1016/j.jpaa.2011.04.004

[6] J.M. Boardman, Modular representations on the homology of power of real projective space, in Algebraic Topology: Oaxtepec 1991, ed. M. C. Tangor; in Contemp. Math. 146 (1993), 49-70. http://dx.doi.org/10. 1090/conm/146

[7] A.K. Bousfield, E.B. Curtis, D.M. Kan, D.G. Quillen, D.L. Rector and J.W. Schlesinger, The mod-p lower central series and the Adams spectral sequence, Topology 5 (1966), no. 4, 331-342. https://doi.org/10.1016/ 0040-9383 (66) 90024-3

[8] W. Browder, The Kervaire invariant of framed manifolds and its generalization, Ann. of Math. (2) 90 (1969), 157-186. https://doi.org/10.2307/1970686

[9] R.R. Bruner, L.M. Hà and N.H.V. Hưng, On behavior of the algebraic transfer, Trans. Amer. Math. Soc. 357 (2005), no. 2, 437-487. https://doi.org/10.1090/S0002-9947-04-03661-X

[10] T.W. Chen, Determination of $\operatorname{Ext}_{\mathscr{A}}^{5, *}(\mathbb{Z} / 2, \mathbb{Z} / 2)$, Topol. Appl. 158 (2011), no. 5, 660-689. https://doi.org/ $10.1016 / j$.topol.2011.01.002

[11] P.H. Chơn and L.M. Hà , Lambda algebra and the Singer transfer, C. R. Math. Acad. Sci. Paris 349 (2011), no. 1-2, 21-23. https://doi.org/10.1016/j.crma.2010.11.008

[12] M.C. Crabb and J.R. Hubbuck, Representations of the homology of BV and the Steenrod algebra II, in Algebra Topology: New trend in localization and periodicity; in Progr. Math. 136 (1996), 143-154. https://doi.org/ 10.1007/978-3-0348-9018-2_9

[13] M.D. Crossley, Monomial bases for $H^{*}\left(\mathbb{C} P^{\infty} \times \mathbb{C} P^{\infty}\right)$ over $\mathscr{A}(p)$, Trans. Amer. Math. Soc. 351 (1999), no. 1, 171-192. https://doi.org/10.1090/S0002-9947-99-02060-7

$[14] \_, \mathcal{A}(p)$ generators for $H^{*}(V)$ and Singer's homological transfer, Math. Z. 230 (1999), no. 3, $401-411$. https://doi.org/10.1007/PL00004698

[15] L.M. Hà Sub-Hopf algebras of the Steenrod algebra and the Singer transfer, Geom. Topol. Publ. 11 (2007), 101-124. https://doi.org/10.2140/gtm.2007.11.81

[16] M. A. Hill, M. J. Hopkins and D. C. Ravenel, On the non-existence of elements of kervaire invariant one, Ann. of Math. (2) 184 (2016), 1-262. https://doi.org/10.4007/annals.2016.184.1.1

[17] N.H.V. Hưng and F.P. Peterson, A-generators for the Dickson algebra, Trans. Am. Math. Soc. 347 (1995), no. 12, 4687-4728. https://doi.org/10.1090/S0002-9947-1995-1316852-X

[18] N.H.V. Hưng and T.N Nam, The hit problem for the Dickson algebra, Trans. Am. Math. Soc. 353 (2001), no. 12, 5029-5040. https://doi.org/10.1090/S0002-9947-01-02705-2

[19] - The cohomology of the Steenrod algebra and representations of the general linear groups, Trans. Amer. Math. Soc. 357 (2005), no. 10, 4065-4089. https://doi.org/10.1090/S0002-9947-05-03889-4

[20] A.S. Janfada and R.M.W. Wood, The hit problem for symmetric polynomials over the Steenrod algebra, Math. Proc. Cambridge Philos. Soc. 133 (2002), no. 2, 295-303. https://doi.org/10.1017/S0305004102006059

[21] M. Kameko, Products of projective spaces as Steenrod modules, PhD. thesis, The Johns Hopkins University, ProQuest LLC, Ann Arbor, MI, 1990, 29 pages.

[22] W.H. Lin and M. Mahowald, The Adams spectral sequence for Minami's Theorem, in: Proceedings of the Northwestern Homotopy Theory Conference (Providence, Rhode Island) (H. R. Miller and S. B. Priddy, eds.), Contemp. Math. vol. 220, 1983, pp. 143-177. http://dx.doi.org/10.1090/conm/220

[23] W.H. Lin, $\operatorname{Ext}_{\mathcal{A}}^{4, *}(\mathbb{Z} / 2, \mathbb{Z} / 2)$ and $\operatorname{Ext}_{\mathcal{A}}^{5, *}(\mathbb{Z} / 2, \mathbb{Z} / 2)$, Topol. Appl. 155 (2008), no. 5, 459-496. https://doi.org/ $10.1016 / \mathrm{j}$.topol.2007.11.003

[24] A. Liulevicius, The factorization of cyclic reduced powers by secondary cohomology operations, Proc. Natl. Acad. Sci. USA. 46 (1960), no. 7, 978-981. https://doi.org/10.1073/pnas.46.7.978

[25] Ib H. Madsen, On the action of the Dyer-Lashof algebra in $H_{*}(G)$, Pac. J. Math. 60 (1975), no. 1, 235-275. https://doi.org/10.2140/pjm.1975.60.235

[26] N. Minami, The iterated transfer analogue of the new doomsday conjecture, Trans. Amer. Math. Soc. 351 (1999), no. 6, 2325-2351. https://doi.org/10.1090/s0002-9947-99-02037-1

[27] M.F. Mothebe, Generators of the polynomial algebra $\mathbb{F}_{2}\left[x_{1}, x_{2}, \ldots, x_{n}\right]$ as a module over the Steenrod algebra, PhD. thesis, The University of Manchester, 1997.

[28] M.F. Mothebe and L. Uys, Some relations between admissible monomials for the polynomial algebra, Int. J. Math. Math. Sci., Article ID 235806, 2015, 7 pages. https://doi.org/10.1155/2015/235806

[29] M.F. Mothebe, P. Kaelo and O. Ramatebele, Dimension formula for the polynomial algebra as a module over the Steenrod algebra in degrees less than or equal to 12, Journal of Mathematics Research 8 (2016), no. 5, 92-100. https://doi.org/10.5539/jmr.v8n5p92

[30] T.N. Nam, A-générateurs génériques pour l'algèbre polynomiale, Adv. Math. 186 (2004) no. 2, 334-362. https: //doi.org/10.1016/j.aim.2003.08.004 
[31] _ Transfert algébrique et action du groupe linéaire sur les puissances divisées modulo 2, Ann. Inst. Fourier (Grenoble) 58 (2008), no. 5, 1785-1837. https://doi.org/10.5802/aif.2399

[32] D.J. Pengelley and F. Williams, Global structure of the mod two symmetric algebra, $H^{*}\left(B O, \mathbb{F}_{2}\right)$, over the Steenrod algebra, Algebr. Geom. Topol. 3 (2003), no. 2, 1119-1138. https://doi.org/10.2140/agt.2003.3.1119

[33] _ Beyond the hit problem: Minimal presentations of odd-primary Steenrod modules, with application to $C P(\infty)$ and $B U$, Homology Homotopy Appl. 9 (2007), no. 2, 363-395. https://doi.org/10.4310/HHA.2007. v9.n2.a13

[34] A new action of the Kudo-Araki-May algebra on the dual of the symmetric algebras, with applications to the hit problem, Algebr. Geom. Topol. 11, (2011), no. 3, 1767-1780. https://doi.org/10.2140/agt.2011. 11.1767

[35] - The hit problem for $H^{*}\left(B U(2) ; \mathbb{F}_{p}\right)$, Algebr. Geom. Topol. 13 (2013), no. 4, 2061-2085. https://doi. org/10.2140/agt.2013.13.2061

[36] _ Sparseness for the symmetric hit problem at all primes, Math. Proc. Cambridge Philos. Soc. 158, (2015), no. 2, 269-274. https://doi.org/10.1017/S0305004114000668

[37] F.P. Peterson, Generators of $H^{*}\left(\mathbb{R} P^{\infty} \times \mathbb{R} P^{\infty}\right)$ as a module over the Steenrod algebra, Abstracts Amer. Math. Soc., Providence, RI, April 1987.

[38] _ A-generators for certain polynomial algebras, Math. Proc. Cambridge Philos. Soc. 105 (1989), no. 2, 311-312. https://doi.org/10.1017/S0305004100067803

[39] Đ.V. Phúc and N. Sum, On the generators of the polynomial algebra as a module over the Steenrod algebra, C.R.Math. Acad. Sci. Paris 353 (2015), no. 11, 1035-1040. https://doi.org/10.1016/j.crma.2015.09.002

[40] - On a minimal set of generators for the polynomial algebra of five variables as a module over the Steenrod algebra, Acta Math. Vietnam. 42 (2017), no. 1, 149-162. https://doi.org/10.1007/s40306-016-0190-z

[41] Đ.V. Phúc, The "hit" problem of five variables in the generic degree and its application, Topol. Appl. 282 (2020), 107321, in press. https://doi.org/10.1016/j.topol.2020.107321

$[42] \ldots$ A-generators for the polynomial algebra of five variables in degree $5\left(2^{t}-1\right)+6.2^{t}$, Commun. Korean Math. Soc. 35 (2020), no. 2, 371-399. https://doi.org/10.4134/CKMS.c190076

[43] On the dimension of $H^{*}\left(\left(\mathbb{Z}_{2}\right)^{\times t}, \mathbb{Z}_{2}\right)$ as a module over Steenrod ring, Topol. Appl. 303 (2021), 107856, in press. https://doi.org/10.1016/j.topol.2021.107856

[44] - On Peterson's open problem and representations of the general linear groups, Preprint 2020,61 pages, available online at http://arxiv.org/abs/1907.08768

[45] - On the hit problem for the polynomial algebra and the algebraic transfer, Preprint (2022), 40 pages, available online at https://www.researchgate.net/publication/364695637

[46] A note on the hit problem for the polynomial algebra of six variables and the sixth algebraic transfer, J. Algebra 613 (2023), https://doi.org/10.1016/j.jalgebra.2022.08.028

$[47]$ Structure of the space of $G L_{4}\left(\mathbb{Z}_{2}\right)$-coinvariants $\mathbb{Z}_{2} \otimes_{G L_{4}\left(\mathbb{Z}_{2}\right)} P H_{*}\left(\mathbb{Z}_{2}^{4}, \mathbb{Z}_{2}\right)$ in some generic degrees and its application, Topol. Appl. (2022), accepted for publication, available online at https://www.researchgate. net/publication/350592289

[48] _ A note on the modular representation on the $\mathbb{Z} / 2$-homology groups of the fourth power of real projective space and its application, Preprint (2021), 52 pages, available online at https://www.researchgate.net/ publication/353065445

[49] - On the lambda algebra and Singer's cohomological transfer, Preprint (2021), available online at https: //arxiv.org/abs/2110.00763

[50] - The affirmative answer to Singer's conjecture on the algebraic transfer of rank four, Proc. Roy. Soc. Edinburgh Sect. A (2022), DOI: 10.1017/prm.2022.57, available online at https://www.researchgate.net/ publication/352284459.

[51] _ On modules over the mod 2 Steenrod algebra and hit problems, Preprint (2021), 40 pages, available online at https://www.researchgate.net/publication/365597765

[52] - On the hit problem for the algebra $H^{*}\left(B \mathbb{F}_{2}^{s}, \mathbb{F}_{2}\right)$ as a module over the mod two Steenrod algebra and applications, Preprint (2022), available online at https://osf.io/swdka/

[53] S. Priddy, On characterizing summands in the classifying space of a group, I, Amer. Jour. Math. 112 (1990), no. 5, 737-748. https://doi.org/10.2307/2374805

[54] J. Repka and P. Selick, On the subalgebra of $H_{*}\left(\left(\mathbb{R} P^{\infty}\right)^{n} ; \mathbb{F}_{2}\right)$ annihilated by Steenrod operations, J. Pure Appl. Algebra 127 (1998), no. 3, 273-288. https://doi.org/10.1016/S0022-4049(96)00177-6

[55] J.H. Silverman, Hit polynomials and conjugation in the dual Steenrod algebra, Math. Proc. Cambriges Phil. Soc. 123 (1998), no. 3, 531-547. https://doi.org/10.1017/S0305004197002302

[56] J.H. Silverman and W.M. Singer, On the action of Steenrod squares on polynomial algebras II, J. Pure Appl. Algebra 98 (1995), no. 1, 95-103. https://doi.org/10.1016/0022-4049(95)90027-6

[57] W.M. Singer, The transfer in homological algebra, Math. Z. 202 (1989), no. 4, 493-523. https://doi.org/10. $1007 / \mathrm{BF} 01221587$

[58] - On the action of the Steenrod squares on polynomial algebras, Proc. Amer. Math. Soc. 111 (1991), no. 2, 577-583. https://doi.org/10.1090/S0002-9939-1991-1045150-9

[59] - Rings of symmetric functions as modules over the Steenrod algebra, Algebr. Geom. Topol. 8 (2008), no. 1, 541-562. https://doi.org/10.2140/agt.2008.8.541

[60] N.E. Steenrod and D.B.A. Epstein, Cohomology operations, Annals of Mathematics Studies 50, Princeton University Press, Princeton N.J, 1962.

[61] N. Sum, The negative answer to Kameko's conjecture on the hit problem, Adv. Math. 225 (2010), no. 5, 2365-2390. https://doi.org/10.1016/j.aim.2010.04.026

[62] _ On the Peterson hit problem, Adv. Math. 274 (2015), 432-489. https://doi.org/10.1016/j.aim. 2015.01 .010

[63] - On the Peterson hit problem of five variables and its applications to the fifth Singer transfer, East-West J. Math. 16 (2014), no. 1, 47-62, available online at eastwestmath.org/index.php/ewm/article/view/67.

[64] - On the determination of the Singer transfer, Vietnam Journal of Science, Technology and Engineering, 60 (2018), no. 1, 3-16. https://doi.org/10.31276/VJSTE.60(1).03, available online at https: //vietnamscience.vjst.vn/index.php/VJSTE/article/view/100. 
[65] - On a construction for the generators of the polynomial algebra as a module over the Steenrod algebra, in Singh M., Song Y., Wu J. (eds), Algebraic Topology and Related Topics. Trends in Mathematics. Birkhäuser/Springer, Singapore (2019), 265-286. https://doi.org/10.1007/978-981-13-5742-8_14

[66] _ The kernel of Kameko's homomorphism and the Peterson hit problem, Preprint 2019, 31 pages, available online at http://viasm.edu.vn/xuat-ban/tien-an-pham-viasm/?filter=2019.

[67] _ The squaring operation and the Singer algebraic transfer, Vietnam J. Math. (2020), in press. https: //doi.org/10.1007/s10013-020-00423-1

[68] M.C. Tangora, On the cohomology of the Steenrod algebra, Math. Z. 116 (1970), no. 1, 18-64. https://doi. org/10.1007/BF01110185

[69] N.K. Tín, The hit problem for the polynomial algebra in five variables and applications, PhD. thesis, Quy Nhon University, 2017.

[70] _ The hit problem for the polynomial algebra as a module over Steenrod algebra, I, JP J. Algebra Number Theory Appl. 47 (2020), no. 1, 67-86. http://dx.doi.org/10.17654/NT047010067

[71] G. Walker and R.M.W. Wood, Young tableaux and the Steenrod algebra, in Proceedings of the School and Conference in Algebraic Topology, Hanoi 2004, in Geom. Topol. Monogr. 11 (2007), 379-397. http://doi.org/ $10.2140 / \mathrm{gtm} .2007 .11 .379$

[72] __ Weyl modules and the mod 2 Steenrod algebra, J. Algebra 311 (2007), no. 2, 840-858. http://doi. org/10.1016/j.jalgebra.2007.01.021

[73] _ Polynomials and the mod 2 Steenrod Algebra: Volume 1, The Peterson hit problem, in London Math. Soc. Lecture Note Ser., Cambridge Univ. Press, January 11, 2018.

[74] C.T.C. Wall, Generators and relations for the Steenrod algebra, Ann. of Math. (2) 72 (1960), no. 3, 429-444. http://doi.org/10.2307/1970225

[75] J.S.P. Wang, On the cohomology of the mod-2 Steenrod algebra and the non-existence of elements of Hopf invariant one, Illinois J. Math. 11 (1967), no. 3, 480-490. http://doi.org/10.1215/ijm/1256054570

[76] R.M.W. Wood, Steenrod squares of polynomials and the Peterson conjecture, Math. Proc. Cambriges Phil. Soc. 105 (1989), no. 2, 307-309. https://doi.org/10.1017/S0305004100067797

[77] _ Problems in the Steenrod algebra, Bull. London Math. Soc. 30 (1998), no. 5, 449-517. https://doi. org/10.1112/S002460939800486X

[78] H. Zare, The Dyer-Lashof algebra and the hit problems, New York J. Math. 27 (2021), 1134-1172.

Đạng Võ Phúc

Faculty of Education Studies

UNIVERSITY OF KHANH HOA

01 Nguyen Chanh, Nha Trang, Khanh Hoa, Vietnam

Email address: dangvophuc@ukh.edu.vn 